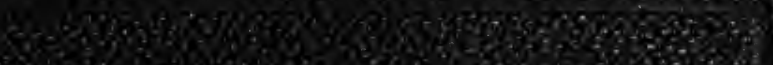

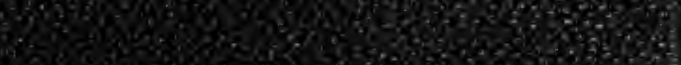

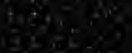

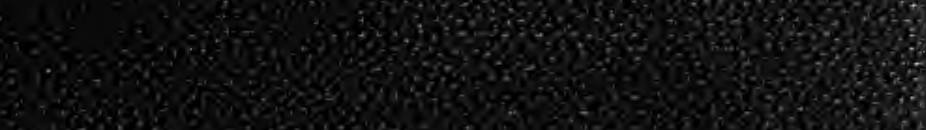
u.

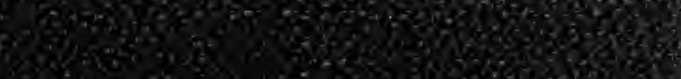
3. 3. 10.

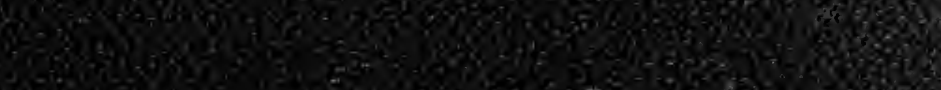
3.

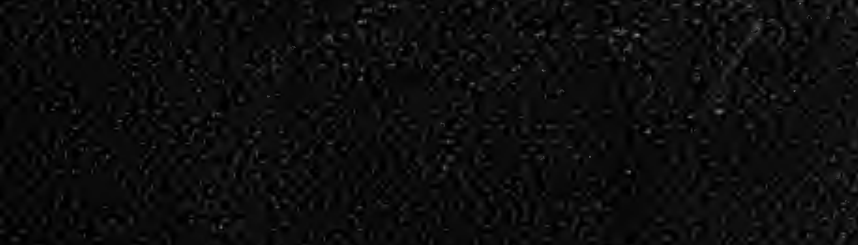

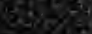
per.

38 $y=0,5$

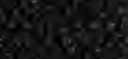

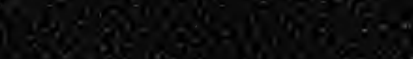

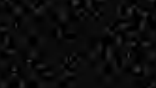
wosest.

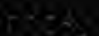

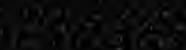

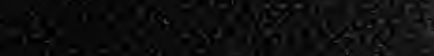

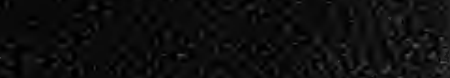
2.030 $\sin 203$

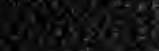

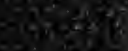
$\operatorname{lin}^{2}=28$ arses 1.98 $8, \sqrt{3}$ 
$5^{0} 2^{0^{0}}$ 
THE SCIENCE OF HUMAN BEHAVIOR 


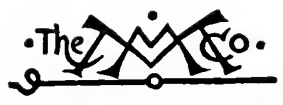

\section{THE MACMILLAN COMPANY \\ NEW YORK - BOSTON - CHICAGO \\ DALLAS - SAN FRANCISCO}

MACMILLAN \& CO., LIMITED

LONDON - BOMBAY - CALCUTTA

MBLBOURNE

THE MACMILLAN CO. OF CANADA, LtD.

TORONTO 
Psyeh.

P254s

\section{THE SCIENCE OF}

\section{HUMAN BEHAVIOR}

\section{BIOLOGICAL AND PSYCHOLOGICAL FOUNDATIONS}

BY

MAURICE PARMELEE, Ph.D.

UNIVERSITY OF MISSOURI

AUTHOR OF "THE PRINCIPLES OF ANTHROPOLOGY AND SOCIOLOGY

IN THEIR RELATIONS TO CRIMINAL PROCEDURE," ETC.

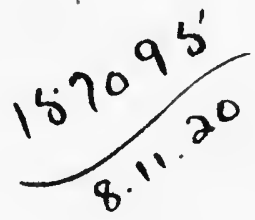

Xjew Hark

THE MACMILLAN COMPANY

I9I6

All rights reserved 


\section{COPYRJGHT, 1913,}

\section{By THE MACMILLAN COMPANY.}

Set up and electrotyped. Published January, rg×3. Reprinted September, $19 x 6$. 


\section{To}

THREE MASTER INVESTIGATORS

\section{JACQUES LOEB - H. S. JENNINGS}

CHARLES S. SHERRINGTON

EACH OF WHOM HAS IN HIS OWN WAY DONE MUCH TO ADVANCE THE SCIENCE OF BEHAVIOR 


\section{Digitized by the Internet Archive in 2007. with funding from Microsoft Corporation}




\section{PREFACE}

THE study of human phenomena must be carried on largely through the psychological and social sciences. Much of the writing in these sciences still consists of vague generalizations which have not been subjected to the test of being applied to a large number of data. What is needed above all is to carry much further the painstaking labor of accumulating a large mass of facts from which generalizations can be safely deduced. In other words, these sciences must get down to concrete realities more than they have in the past if they are to develop on a stable basis.

It is the function of every science, on the one hand, to reduce as far as possible the phenomena with which it deals to the terms of the science upon which it is based, and, on the other hand, to describe the peculiar characteristics of these phenomena which distinguish them from the phenomena out of which they have evolved and which make them a fit subject for treatment by a distinct science. Thus psychical and social phenomena should be reduced as far as possible to biological terms, just as vital phenomena should be reduced as far as possible to chemical and physical terms. On the other hand, the peculiar characteristics of psychical and social phenomena should be described, just as the peculiar characteristics of vital phenomena should be described. Thus, on the one hand, the continuity of the evolutionary series would be 
shown, while, on the other hand, would be illustrated the creative nature of the evolutionary process which is ever bringing into being new combinations of matter and energy or whatever the ultimate elements of the universe may be.

If then the psychologists and social scientists place their work more than ever on a biological basis they will make it more definite and concrete and will get much nearer to the real nature of the phenomena with which they deal. In this book I have brought together the results of recent work in biology in general and in zoölogy and neurology in particular, in genetic and comparative psychology, and in anthropology, and have tried to show the significance of this work for the analysis of human behavior. The book contains certain contributions which will, I hope, be of interest to psychologists, anthropologists, and social scientists. But $I$ hope it will be of value also to many general readers who will be glad to have in convenient form the results of this work which is of so much significance for the study of human behavior and human nature. It should be useful to those engaged in educational work and may also prove to have utility as a college and university textbook in certain courses in psychology and sociology, as, for example, courses in comparative psychology and in biological and psychological sociology.

While a single theme runs through this book, namely, the evolution of behavior, yet the book naturally falls into several parts, each of which may be read with some profit apart from the rest. Chapters II to IV summarize most of the fundamental facts and principles of modern biology. Chapters V to VII describe briefly the behavior of the lower animals. Chapters VIII to $\mathrm{X}$ give some of the most important facts and principles of neurology. Chapters XI 
to XVI cover the whole field of psychology in a concise fashion and, in fact, form in themselves a small treatise on psychology. Chapters XVII to XX state the most important facts with regard to the beginnings of social evolution and the fundamental forces in social evolution.

This book is the first of a series of works in which I propose to deal with the evolution of human culture and of human nature on the basis furnished by this book.

I wish to thank my colleagues in the University of Missouri, Professors W. C. Curtis, D. H. Dolley, C. A. Ellwood, J. W. Hudson, George Lefevre, and Max Meyer, each of whom.has read one or more of the chapters and has offered comments and criticisms thereon.

I wish also to thank the following publishing firms, P. Blakiston's Son and Company, The Columbia University Press, Henry Holt and Company, and J. B. Lippincott and Company, each of which has given me permission to use illustrations which have appeared in books published by it.

MAURICE PARMELEE.

December, I9I2. 
,

$\because 3-$

$\because \because$ 


\section{CONTENTS}

\section{CHAPTER I \\ THE STUDY OF BEHAVIOR}

Definition of behavior - The science of behavior in relation to biology, psychology, and sociology - The genetic method of study - Plan of this book . . . . . . . . . .

\section{CHAPTER II}

\section{THE PHYSICO-CHEMICAL BASIS OF BEHAVIOR}

Physical and chemical characteristics of matter - Reasons for the mobile and plastic character of organic matter - The origin of the organism - The cell-The constituents of organic matter . .

\section{CHAPTER III}

\section{THE ANATOMICAL AND PHYSIOLOGICAL BASIS OF BEHAVIOR}

Metabolism - Respiration - Circulation - Reproduction - Sex Theories of the origin of species - Orthogenesis - Nägeli- Eimer - Differentiation of species through the action of external forces Lamarck - Adaptation through the transmission of acquired characters - Darwin - Variation - Selection - Criticisms of Darwinism - Weismann - Inheritance through the germ cell - Germinal selection-Panmixia . • . . . . . • . • .

\section{CHAPTER IV}

\section{THE ANATOMICAL AND PHYSIOLOGICAL BASIS OF BEHAVIOR (Continued)}

Heterogenesis - Discontinuous variations - Bateson - Substantive and meristic variations - Symmetry - De Vries - The mutation theory - Micromeric theories of the germ cell - Unit characters Mendel - The Mendelian law of inheritance - Dominant and recessive characters - The segregation of allelomorphic characters Homozygotes and heterozygotes - The Mendelian explanation of particulate, exclusive, and blended inheritance-Reversion or ata- 
vism - Galton's laws of ancestral inheritance and of filial regression - Mendelian criticism of Galton's laws - Statistical and physiological laws of inheritance - The exaggerated claims for Mendelism - Johannsen's theory of pure lines - Sex determination - Sex as a unit character-The biological basis for psychology, anthropology, and sociology

\section{CHAPTER V}

\section{THE BEHAVIOR OF THE LOWER ANIMALS}

Behavior as the dynamic, functional aspect of external organic phenomena-Application of the genetic method in psychology The genetic method as the phylogenetic method-The development of the. science of behavior by biologists and comparative psychologists - Experimental psychology - The irritability of organic matter arising out of its unstable and mobile nature - Is this irritability a psychic characteristic ? - The direct reactions of organisms to external forces - Definition of a tropism - The external forces which act upon organisms ..$\quad$. . . . . .

\section{CHAPTER VI}

\section{TROPISMS}

Phototropism - Photopathy - Does the direction or the intensity of the rays of light determine the reactions of organisms to light?Positive and negative phototropism - Is the action of light on organic matter mechanical or chemical ? - Chromotropism - Rudimentary vision - The utility of reactions to light-Geotropism - The force of gravity - Chemotropism - Rudimentary senses of smell and taste - Galvanotropism - Barotaxis - Stereotropism (thigmotropism) or contact irritability - Rheotropism-Anemotropism - Thermotropism-Hydrotropism - Tonotropism . • . ..

\section{CHAPTER VII}

\section{THE EVOLUTION OF ANIMAL BEHAVIOR}

Determination of behavior by form and past experience and by external stimuli-Description of the behavior of Amœba, Paramecium, Stentor, etc. - The action system - The reflex - The physiological state of the organism as a factor in the determination of behavior - The regulation of behavior - The development of behavior in the race-Organic selection - The laws of animal behavior 


\section{CHAPTER VIII}

\section{THE EVOLUTION OF THE NERVOUS SYSTEM AND THE REFLEX ACTION}

Progressive increase in the self-determination of behavior - Nerve cells and fibers - The specific qualities of nervous matter - Definitions of the reflex action - Receptor, adjustor, and effector organs - The simple reflex action - The conductibility of nervous matter - The adaptive character of reflex actions - The origin of the nervous system-The appropriation of effectors by the nervous system - The selective excitability of receptor organs - The neurone - The connections between the neurones - The central nervous system.

\section{CHAPTER IX}

\section{THE FUNCTIONS OF THE NERVOUS SYSTEM}

The kinds of reflex arcs - Facilitation - Inhibition - The common path - The integrative function of the nervous system - Mechanical, chemical, and nervous integration-The functional divisions of the nervous system - The origin of the head and brain - The functions of the distance receptors - Extero-ceptive, proprio-ceptive, and intero-ceptive receptors - The neuromeres - The divisions of the brain - The functions of the cerebellum - The functions of the cerebrum - The divisions of the cerebrum . . . . . .

\section{CHAPTER X}

\section{CEREBRAL LOCALIZATION}

Phrenology - Anatomical study of cerebral functions - The anatomy of the brain - White and gray matter - The cerebral cortex Theories of cerebral localization - The association areas-The cerebral fibers-Physiological study of the cerebral functions by stimulation and extirpation - Clinico-pathological study of cerebral functions - Functional and organic diseases of the brain . .

\section{CHAPTER XI}

\section{THE NATURE OF INSTINCT}

Mistaken conceptions of instinct-Instincts as congenital tendencies to action- The relation of instinct to tropism and reflex action - The adaptive nature of instincts - The structural basis of instincts 
- The hereditary nature of instincts - Instincts as characterizing species - The degree of permanency of instincts - Variations in instincts - Reënforcement and inhibition-Combinations of instincts - The degree of perfection attained by instincts - Instinct and consciousness - The relation between instincts and emotions-Definitions of instinct-An instinct as an inherited combination of reflexes.

\section{CHAPTER XII}

\section{THE NEURAL BASIS OF INSTINCT}

The cerebrum and instinct-The cerebellum as the principal nervous mechanism for instincts - Localization of instincts in the spinal cord, medulla, and cerebellum - Impulsive instincts - Chain instincts - Analysis of the nesting instinct of solitary wasps . .

\section{CHAPTER XIII}

\section{THE PRINCIPAL HUMAN INSTINCTS AND GENERAL INNATE TENDENCIES}

The number of human instincts - The directing of instinctive tendencies by intelligence-Description of the principal human instincts - The classification of the instincts - The nature of a general innate tendency - Imitation - Suggestion - Sympathy - Play - Emulation - Workmanship - Gregariousness - Habit . . . . .

\section{CHAPTER XIV}

\section{THE NATURE OF INTELLIGENCE}

Psycho-physical processes - Introspection - Intelligent behavior as the result of experience-Associative memory as a criterion of intelligence - Unspecialized nervous tissue essential for intelligence - The cerebral cortex as the neural basis for intelligence-The central nervous system as the organ of memory - The range of intelligence in the animal world - The structural advantages of the vertebrate for the development of intelligence - The reasons for man's superior intelligence - The nature of learning - Sensations - Images-Memory-Sensori-motor and ideo-motor action-Pleasure and pain as reënforcing and inhibiting ideo-motor action-Ideas Thought as a flow of ideas - Concepts as generalized imagesImageless thought - Reason - The nature of human intelligence .. 


\section{CHAPTER XV}

CONSCIOUSNESS : SENSATION, ATTENTION, FEELING, PLEASURE, PAIN, AND EMOTION AS

\section{CONSCIOUS ELEMENTS}

Spiritual conceptions of consciousness - Consciousness as characterizing all matter-Consciousness as characterizing all organic matter-Consciousness as an epiphenomenon-Consciousness as associative memory - The neural basis of consciousness - The relation between intelligence and consciousness - Sensations as the raw material for consciousness - The nature of attention - The nature of feeling - Pleasure and pain-Feelings as pleasurable and painful sensations - The neural basis of feelings - Feelings of pleasantness and unpleasantness - The nature of emotion - The visceral and vaso-motor origin of emotions - Emotions as affective sensations or feelings .

\section{CHAPTER XVI}

\section{PERSONALITY, INTELLIGENCE, CONSCIOUSNESS,} AND THE NATURE OF MIND

Self-consciousness or personality based upon the integrated permanent psychic elements - Self-consciousness as an idea - Multiple personality - The nature of volition - Subjective and objective aspects of consciousness - The relation between consciousness and intelligence - The criterion of consciousness - The functions of consciousness - Consciousness as self-consciousness - Definition of consciousness - Psycho-physical parallelism - Psycho-physical interactionism - Definition of mind-Mind as including intelligence and consciousness - Mental processes determined by minute physiological processes - Mind as a stage in the determination of certain kinds of behavior - The relation between mind and matter-The criterion of mind-Is our knowledge of mind subjective or objective?

\section{CHAPTER XVII}

\section{THE BEGINNINGS OF SOCIAL EVOLUTION}

Definitions of association and society - Colonial species - Is there an inborn associative tendency? - The external, environmental forces for association-The degree of correlation between organic and social evolution - Internal-forces for association - Inborn and ac-

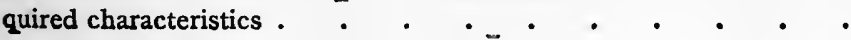




\section{CHAPTER XVIII}

\section{INSECT SOCIETIES: THE ANTS}

Anatomical polymorphism - Physiological division of labor - The philoprogenitive instincts - The founding of an ant communityThe food-procuring activities - The hunting stage - The agricultural stage - Harvesting - The pastoral stage - Symbiosis - Parasitism - Myrmecophilism - Commensalism - Slave-making - The intelligence of ants - The ant brain - Recognition - Communication-Suggestion and imitation-Coöperation-Insect societies are based mainly upon instincts . $. \quad . \quad . \quad . \quad . \quad . \quad$.

\section{CHAPTER XIX}

\section{VERTEBRATE SOCIETIES}

Paleontological evidence of association among vertebrates - Fishes - Amphibians - Reptiles-Difference between cold- and warmblooded vertebrates - Birds - The family - Mammals - Primates Man

\section{CHAPTER XX}

\section{THE FACTORS OF SOCIAL EVOLUTION}

The causes of association - The polyphyletism of animal societies - Utility for survival as a controlling factor in social evolution-The reasons for man's superior social evolution-Environmental forces for association - Instinctive forces for association - Is there a gregarious instinct? - The sexual instinct-The reproductive instincts - The parental instincts - The utility of parental care - Conjugal relations - The family - Wider forms of association - The antagonism between the family and the horde - The family as preparing the way for wider forms of association - Emotional forces for association - Intelligent forces for association - Imitation - Recognition - Communication - Language - The formation of categories Meeting-places - Leadership - Theories of social evolution - Theory of the instinctive origin of society : Petrucci, McDougall - Theory of the emotional origin of society : Adam Smith, SutherlandTheory of the intellectual origin of society: Giddings, Kropotkin, Tarde, Durkheim - The complexity of the factors in social evolution

\section{CHAPTER XXI}

\section{CONCLUSION}

Summary of the preceding chapters - This book furnishes a basis for the study of the more complex human, mental, and social phenomena 


\section{LIST OF FIGURES}

nG.

I. Euglena Virmis

2. Positive Chemotropism . • . • . • . IIo

3. Negative Chemotropism . . . . . . IIo

4. Stereotropism, or Contact Irritability . . . II3

5. Amcba Proteus . . . . . . . . . . II9

6. PARAMEcium . . . . . . . . . 122

7. Stentor Røselit . . . . . . . . . . 125

8. Diagram of a Typical Neurone . . . . . . I55

9. Diagram of a Reflex Arc . . . . . . . $\quad$. $\quad$ I59

io. Diagram of the Central Nervous System facing i66

iI. Diagram of the Convex Surface of the Cerebral

HeMisphere . . . . . . . facing 182

12. Diagram of the Medial Surface of the Cerebral

HEMISPHERE $\quad$ • $\quad$ • . . . facing 182 



\title{
THE SCIENCE OF HUMAN BEHAVIOR
}

\author{
CHAPTER I \\ THE STUDY OF BEHAVIOR
}

Definition of behavior, 1. - The science of behavior in relation to biology, psychology, and sociology, 2. - The genetic method of study, 3. - Plan of this book, 4 .

THE word "behavior" has a variety of meanings. Sometimes it is used in a very limited sense to refer to matters of deportment and etiquette. Sometimes it is used in a very broad sense to refer to the mode of acting of not only living beings, but also inanimate things such as an engine or a waterfall. Recently, however, there has been growing up a so-called "science of animal behavior" which has given to the word a very definite meaning.

This science is being developed on the one hand by zoologists and on the other hand by comparative psychologists. These scientists are studying those visible movements of the animal organism which constitute the external physiological processes. They are trying to explain these movements on the basis of anatomical structure and internal physiological processes stimulated by external forces. In other words, they are searching for a mechanical explanation of these movements. Their method of study 
is therefore thoroughly objective rather than subjective in its character. This is very important in such a comparative study of different species. Otherwise there would be great danger that the explanation of the movements of animals other than man would be too anthropomorphic. It is for this reason that the word "conduct" could not very well be applied to these movements, because this word has acquired an ethical connotation and is therefore highly anthropomorphic in its significance. The word "behavior" has no such connotation and is therefore the best one to apply to these movements.

The science of behavior has already been claimed by several psychologists as constituting a part or the whole of psychology. But a little reflection will indicate that this cannot be so. The study of behavior must be, to begin with and fundamentally, biological, for, as we have seen, it involves the study of anatomical structure and physiological processes. In the second place, it is psychological, for mental forces are frequently involved in determining behavior. In the third place, it is sociological, for much behavior is influenced by the association of living beings. So that the science of behavior can be claimed by no one of these sciences to the exclusion of the others. It is a sort of hybrid product of these three sciences, and as such cannot be regarded as a primary science. But it is useful to call it a science as being a systematic study of these important phenomena. As a science it includes a small part of biology, but most if not all of psychology and sociology, for most if not all of mental and social phenomena can be reduced to terms of behavior.

To begin the study of behavior from a biological point of 
view has, I believe, a very wholesome effect, for it necessitates the use of more or less exact methods of observation which are not always used in psychology and sociology. The use of these methods results in the disappearance of hazy and mystical explanations of human phenomena frequently proposed by writers in these two sciences. These explanations are replaced by more or less exact mechanical explanations.

Furthermore, the genetic method of study involved is valuable for several reasons. It emphasizes the fact of evolution, which is as true of behavior as it is of all other phenomena. It makes use of the comparative method, by means of which much more can be learned about the behavior of a certain kind of animal than if the behavior of that species alone is studied. It resolves the complex forms of behavior into their simpler elements, thus making them much easier to understand. It thus makes many experiments unnecessary and at the same time shows what experiments should be made.

In this book I have tried to lay the foundations for the study of human behavior. This has involved bringing together, so far as possible within one book, the pertinent data from the three sciences of biology, psychology, and sociology. The book is, therefore, in a sense an introduction both to psychology and to sociology. It has been written in the main from the objective point of view of the students of animal behavior. At the same time I do not think it possible to discuss human behavior and ignore the subjective side entirely. Consequently, at certain points, with the proper reserve and qualifications, I have made use of the subjective point of view. I have done so, 
however, without discussing the fundamental philosophical question as to the nature of knowledge. I am well aware that in one sense it is true that all knowledge is subjective. That is to say, all our knowledge comes to us through our senses in the form of sensations, and we cannot be absolutely certain that these sensations represent to us truly the nature of the world which is exterior to us. For scientific purposes, however, we need to practice what is sometimes called "naive realism" and assume that things in the exterior world are actually as our senses represent them to be.

In order to indicate the scope of this book I will outline its contents briefly. The next chapter discusses the composition of organic matter, showing how its mobile and unstable nature makes possible the plasticity of living organisms, thus furnishing a basis for the evolution of behavior. The third and fourth chapters furnish a brief survey of organic evolution, showing how the structural forms and physiological processes which condition behavior have evolved and what forces are at work in the animal world, such as heredity, variation, selection, etc. The next two chapters deal with the behavior of animals without a nervous system, the fifth chapter describing the irritability of organic matter and the external forces which act upon organisms, and the sixth chapter dealing with tropisms or the direct reactions of these lower animals to these external forces. The seventh chapter describes the factors at work in the evolution of behavior, with illustrations from the behavior of the lower animals.

The next three chapters deal with the nervous system, describing briefly the evolution of the nervous system, the 
reflex action, which is the principal type of behavior determined by the nervous system, the functions of the nervous system, and the localization of these functions. The following three chapters deal with instinct as one of the principal types of behavior. In the eleventh chapter instinct is described as an inherited combination of reflexes integrated by the central nervous system. In this and the next chapter are described the structural basis of instinct, variations in instincts, and the different kinds of instincts. The thirteenth chapter discusses the principal human instincts and the general innate tendencies, such as imitation, play, habit, etc.

The fourteenth chapter treats of intelligent behavior as behavior varied in response to experience. This involves a discussion of the different theories as to the nature of intelligence and a review of the experiments which have been made as to the range of intelligence in the animal world. In the fifteenth chapter are discussed the theories as to the nature of consciousness and sensations, feelings, emotions, and ideas as contents of consciousness. In the sixteenth chapter are discussed self-consciousness, the relation between intelligence and consciousness, and the nature of mind. An attempt is made to show the inherent nature of mental or psychic phenomena and to point out the significance of these phenomena for behavior. Throughout these three chapters on intelligent and conscious phenomena the causes for the superior mental development of man are pointed out whenever they can be discerned.

The next four chapters deal with social phenomena. In the seventeenth chapter are discussed the nature of association and the external and internal forces which 
caused the beginnings of social evolution. The eighteenth chapter deals with insect societies and in particular the ants, illustrating polymorphism or more particularly the physiological division of labor which results from it as a cause of association. The nineteenth chapter describes the kinds of societies existing among the different classes of the vertebrates with special reference to the mammalian and primate societies to which human society is most closely related. In the twentieth chapter are discussed the environmental, instinctive, emotional, and intelligent causes of association and the theories which have been proposed as to the origin of society. The concluding chapter summarizes very briefly the ground covered in this book.

In these chapters, then, are described the different types of behavior, and in all of them, and especially in those on social behavior, the attempt is made to show how they are combined in the complex behavior of the higher animals and especially of man. But to analyze this combination is a very difficult thing to do, and only the bare beginning of such an analysis is made in this book. As the study of behavior is continued, this analysis of the combination of the different types of behavior can be carried further, but it can probably never be carried to the same degree of precision as the analysis of certain other kinds of natural phenomena, such as physical and chemical phenomena. 


\section{CHAPTER II}

THE PHYSICO-CHEMICAL BASIS OF BEHAVIOR

Physical and chemical characteristics of matter, 7. - Reasons for the mobile and plastic character of organic matter, I2. - The origin of the organism, I6. - The cell, I6. - The constituents of organic matter, $\mathbf{1 7}$.

ALL forms of matter respond to external forces in the sense that changes take place in them when these forces act upon them. But this is peculiarly true of organic matter in the sense that in response to external forces living organisms will go through movements which are sometimes very complex in their character. As we have seen in the last chapter, these movements constitute behavior. In order to understand the mobile and plastic character of organic matter, which makes behavior possible, it is advisable to consider some of the characteristics of matter in general.

\section{Characteristics of Matter}

The two sciences that deal with matter in general are physics and chemistry. Physics deals with matter in its simplest form. According to the physical sciences the fundamental entities to which all things can be reduced are matter and energy. To these is sometimes added ether, but of the existence of ether as a distinct and separate entity we have as yet no proof, and it may be no more 
than a rarefied form of matter. Outside of these entities all else is more complex in character and can be reduced to these entities. As to whether these entities are irreducible we do not yet know, but it is possible that matter can be reduced to energy. It is also possible that energy can be reduced to something more simple, in which case neither matter nor energy is a fundamental entity.

Chemistry deals with matter in its complex forms. Matter usually manifests itself to the senses in the form of compounds, and chemistry is occupied with reducing these compounds to their elementary parts. About eighty of these elements have been distinguished, and so far as we know every compound in the universe is made up of a combination of some of these elements. The unit of each of these elements is called its atom. The smallest amount of an element or of a compound composed of two or more elements which can exist in a free state is called a molecule.

For a long time it was thought that the atom of each element was irreducible and that these elementary atoms were ultimate forms of matter. This belief was effectually shaken by the discovery of the periodic law. This law was discovered as a result of a comparison of the weights of these elementary atoms. It was found that when these atomic weights are arranged in tabular form, as, for example, on the basis of hydrogen as one, or of oxygen as sixteen, many of them are even numbers or almost even numbers. The first hypothetical explanation of this was that each of the atoms is made up of a combination of atoms of hydrogen, which is the lightest element. Not all 
of the atomic weights, however, are even numbers when the hydrogen atom is reckoned as one, so that hydrogen could not be regarded as the ultimate form of matter. But it was discovered that the elements when arranged in the order of their atomic weights naturally fall into groups of elements whose behavior is similar. These similarities and relationships seem to indicate that these elementary atoms could not be irreducible units, each of them absolutely distinct from and unrelated to all the others. On the contrary, it seemed very evident that there must be a unit or units smaller than the smallest of the elementary atoms out of which these atoms are formed, and that the similarities of these atoms must be explained by the similarity of their component parts.

The fact of subatomicity or of the existence of particles smaller than atoms was thus made highly probable. But these particles are of necessity so small that for some time it was impossible to secure tangible evidence of their existence, even with the most delicate instruments. Such evidence was finally secured through a study of the conduction of electricity by gases. It was found that electricity is conducted through gases by means of very small particles. These particles were named ions, those carrying negative electricity being called corpuscles or electrons. Little is known as yet with regard to the nature of positive electricity, but it is believed that the ions carrying particles of positive electricity are nearly as large as an atom. "The positive ion consists of an atom of the electrolyte with one of its corpuscles missing. In this way a unit of negative electricity is removed from it, that is, it is left with a posi- 
tive charge." 1 The negative ions or corpuscles or electrons are of greater interest to us, for they represent the smallest particles of matter of which we have tangible evidence.

But the discovery that electricity is carried by these small particles of matter did not reveal the nature of electricity itself, unless electricity is nothing more than these particles. That electricity is matter decomposed to an elementary form seems to be indicated by the fact that it possesses a fundamental property of matter, namely, inertia. This is asserted by an English physicist in the following words: "My first thesis is that an electric charge possesses the most fundamental and characteristic property of matter, viz., mass or inertia; so that if any one were to speak of a milligramme or an ounce or a ton of electricity, though he would certainly be speaking inconveniently, he might not necessarily be speaking erroneously." 2 This may be stated just as well conversely, by saying that matter is electricity.

Along with the development of the electrical theory of matter came the discovery of the new element radium. This element was found to possess the characteristic of radioactivity, or the power of emitting minute particles of matter. It was found also that these radiations were caused by a slow process of decomposition in the atom of radium, in the course of which the radium was becoming transformed into other elements. This discovery of the possibility of transformation among the elements raised the question whether matter in general is not unstable and in process of transformation. "Is matter in general under-

1 W. C. D. Whetham, The Recent Development of Physical Science, Philadelphia, 1904, p. 190. 2 Oliver Lodge, Modern Views on Matter, Oxford, 1903, p. 4. 


\section{The Physico-Chemical Basis of Behavior 11}

going transformation? The raising of such a question would, until a few years ago, have been regarded as extraordinary, since the elements were regarded as stable and unchanging. In the light of the recent investigations with the radioactive elements it is most pertinent. There is some evidence, as we shall see, that all the elements are radioactive to a very small extent. If this should be proved to be due to the elements themselves, to be a property inherent in all matter, and not caused by the deposition of some form of radioactive matter, then, from what has been said above, we must regard matter in general as undergoing change. This change is slow, very slow, but is progressing continuously; the more complex, unstable forms breaking down into simpler aggregates of electrons. If it should be shown that all matter is slightly radioactive, as seems not improbable, then we should be forced to the conclusion of the general instability of the chemical elements." 1

- The belief that matter is radioactive is based upon the electronic or corpuscular theory of matter, for transformations in the atoms of the chemical elements would not be possible if the elements were simple and were not composed of still smaller units. Furthermore, it is necessary to assume that there is a definite arrangement of the smaller units within each atom, and that when this arrangement is disturbed the atom is destroyed and that the atom of another element is formed. "The electronic theory of matter supposes that an atom is composed of a swarm of electrons in rapid movement held in equilibrium

1 Harry C. Jones, The Electrical Nature of Matter and Radioactioity, New York, I906, p. I6r. 
by the internal forces of the atom. In the case of heavy atoms, like those of the radioelements, it is not necessary to suppose that each of these electrons has complete freedom of movement. The character of the transformation of the atom suggests that it is built up in part of a number of secondary units, consisting of groups or aggregates of electrons in equilibrium, which are in rapid independent motion within the atom." 1

The corpuscular nature of the atom and the radioactivity of matter indicate that the elements are not immutable and that the atom is not the simplest form of matter. In all probability we shall never know the ultimate nature of matter, and this brief review of recent work in physics and chemistry emphasizes the mutable character of all the phenomena of which we can have any knowledge. It is therefore of utility in preparing the way for the study of that process of change in certain forms of matter which we call organic evolution.

\section{Organic Matter}

Let us now turn to the branch of chemistry which is of special significance for organic evolution, namely, organic chemistry. This branch of chemistry was named organic when it was believed that there is an absolute distinction between organic and inorganic matter. "The word 'organic' has now only a historic meaning, being derived from a time - the beginning of the last century - when it was thought that the substances which occur in organized nature, in the animal and vegetable kingdoms, could only be formed under the influence of a special, obscure

1 E. Rutherford, Radioactive Transformations, New York, I906, p. 269. 


\section{The Physico-Chemical Basis of Behavior 13}

force, called the vital force." 1 This belief was destroyed by the discovery of the possibility of forming organic compounds from inorganic materials by synthetic methods. This was first accomplished in 1828 when the organic compound urea was synthesized from inorganic sources by Woehler. Since then many organic compounds have been synthesized, and it is no longer believed that any special vital force is necessary for the formation of organic matter. Organic chemistry is now frequently called the chemistry of carbon compounds, because all organic compounds contain carbon.

But while there is no absolute distinction between inorganic and organic matter, each has certain characteristics which differentiate it from the other, and we must study the peculiar characteristics of organic matter because it is these peculiarities which have made biological, mental, and social evolution possible. These characteristics arise primarily out of certain peculiarities of the principal elements in organic matter. The first of these is allotropism, or the capability of assuming different forms. For example, carbon presents itself as diamond, graphite, and amorphous carbon, while oxygen appears under certain conditions as ozone. These allotropic forms of an element are apparently the result of different arrangements of the atoms within the molecules of the element. Analogous to allotropism in these elements is isomerism in many organic compounds. The isomeric compounds assume different forms, apparently as the result of different arrangements of the molecules of the elements within the molecules of the

1 A. F. Holleman. A Textbook of Organic Chemistry, translated from the second Dutch edition by A.J. Walker, New York, r903, p. I. 


\section{The Science of Human Behavior}

compound. These two peculiarities tend to make organic matter less stable and more subject to change than inorganic matter. Furthermore, they tend to make organic matter mobile.

But there are other properties and forces which promote the instability and mobility of organic matter. "Of the four chief elements which, in various combinations, make up living bodies, three are gaseous under all ordinary conditions and the fourth is a solid. Oxygen, hydrogen, and nitrogen are gases which for many years defied all attempts to liquefy them, and carbon is a solid except perhaps at the extremely high temperature of the electric arc. Only by intense pressure joined with extreme refrigerations have the three gases been reduced to the liquid form." 1 That three of the four principal organic elements are gaseous is of great significance with respect to the mobility of organic matter, for the mobility of the molecules of these elements promotes the mobility of organic matter. The dissimilarities of the organic elements are also of significance in this connection. "These four elements of which organisms are almost wholly composed, exhibit certain extreme unlikenesses. While between two of them we have an unsurpassed contrast in chemical activity; between one of them and the other three, we have an unsurpassed contrast in molecular mobility. While carbon, until lately supposed to be infusible and now volatilized only in the electric arc, shows us a degree of atomic cohesion greater than that of any other known element, hydrogen, oxygen, and nitrogen show the least atomic cohesion of all elements. And while oxygen displays, alike in the range and intensi-

${ }^{1}$ Herbert Spencer, The Principles of Biology, New York, I900, Vol. I, p. 3. 


\section{The Physico-Chemical Basis of Behavior 15}

ties of its affinities, a chemical energy exceeding that of any other substance (unless fluorine be considered an exception), nitrogen displays the greatest chemical inactivity." 1 As organic matter is made up of unlike elements, it is unstable, because these elements are easily separable by external forces. This instability causes mobility and the capacity to differentiate and integrate which make adaptation and biological evolution possible.

On the other hand, the more complex forms of organic matter must have a certain amount of stability in order to make possible the growth of structures more or less permanent and rigid in character. "We may clearly see the necessity for that peculiar composition which we find in organic matter. On the one hand, were it not for the extreme molecular mobility possessed by three out of the four of its chief elements; and were it not for the consequently high molecular mobility of their simpler compounds; there could not be this quick escape of the waste products of organic action; and there could not be that continuously active change of matter which vitality implies. On the other hand, were it not for the union of these extremely mobile elements into immensely complex compounds, having relatively vast molecules which are made comparatively immobile by their inertia, there could not result that mechanical fixity which prevents the components of living tissue from diffusing away along with the effete matter produced by decomposition." 2

We have been discussing the chemical properties of the organic elements which cause the instability and mobility of organic matter. The question may naturally be

1 H. Spencer, op. cit., Vol. I, p. 5.

${ }^{2}$ Ibid., Vol. I, p. 22. 
raised whether the recent discoveries in physics are not of significance in this connection. The discovery of radioactivity and of the electrical nature of matter revealing the fact of subatomicity has shown us that the chemical elements are much more complex than was formerly supposed. Whether, however, this is of peculiar significance for the organic elements, we cannot yet know. If it should be found that the subatomic arrangement of the organic elements is more complex than that of the inorganic elements, we might have a further explanation for the unstable and mobile character of organic matter.

\section{Origin of the Organism and the Cell}

Let us now consider the origin of the organism. The formation of the simplest organisms is probably comparable to the formation of crystals. In crystallization certain molecules which have been brought in close proximity with each other are drawn together by attractive forces which are inherent in them, thus forming crystals which are usually more or less symmetrical in shape. In similar fashion probably originated the primordial protoplasm out of which have evolved all organic forms. But the elements which constitute organic matter possess chemical properties which make it more complex, more mobile, and more responsive and adaptive to its environment than most inorganic compounds, thus differentiating it from the ordinary crystal.

The protoplasmic unit is the cell. The lowest organisms are unicellular, while the higher organisms are multicellular or made up of compounds of the unitary cell. The cell must have originated in the sea, for nowhere else could 


\section{The Physico-Chemical Basis of Behavior 17}

the lowest forms of life have secured nutrition. ${ }^{1}$ On land an organism is not likely to come into direct contact with its food, and therefore needs sense organs to locate its food and means of locomotion to bring itself into contact with it. But in the sea the organism is constantly drifting into contact with food ingredients which are either dissolved in the water or in the form of solids. Then takes place the process of assimilation, metabolism, or nutrition, as it is variously called, when the food is absorbed into the organism. Metabolism is primarily a phenomenon of chemical affinity. The chemical constituents of the organism have an affinity for the chemical constituents of the food, and therefore the organism absorbs the food. As the organism becomes more complex, metabolism becomes something more than a phenomenon of chemical affinity, because special organs within the organism are devoted to the assimilative process.

That life originated in the sea is indicated by the fact that the constituent elements of sea water and air are approximately the same as the constituents of living matter. Sea water contains usually oxygen, nitrogen, carbon, hydrogen, sodium, magnesium, potassium, calcium, chlorine, sulphur, and bromine. Air contains nitrogen, oxygen, and carbonic acid. The necessary constituents of living matter which occur in every cell are oxygen, nitrogen, carbon, hydrogen, sodium, potassium, phosphorus, sulphur, calcium, magnesium, iron, and chlorine. ${ }^{2}$ In addition to

1 Cf. Carl Snyder, The Physical Conditions at the Beginning of Life, in Science Progress, III, I (April, 1909), pp. 579-596. Snyder thinks that life did not originate in the sea, but that most of organic evolution has taken place in the sea. He does not state where else than in the sea life could have originated.

2 Max Verworn, General Physiology, London, I899, pp. 99-102. 
these there occur in some cells silicon, fluorine, bromine, iodine, aluminum, and manganese, while sometimes there occur slight traces of certain metals such as copper. It will be noted that all of these necessary elements except iron and phosphorus are to be found usually in sea water and air, while a certain amount of iron and phosphorus must be dissolved in the water at the time living matter is generated. From the air, which is always in contact with the water and sometimes mingled with it, are derived some of the constituents of living matter. Organic evolution must have begun when under favorable conditions ${ }^{1}$ there was formed in the sea for the first time the primordial protoplasm out of which all organic forms have evolved. At first this protoplasm was, in all probability, a formless, jellylike mass. Then out of it there evolved the organic cell, which is colloidal rather than crystalline in its character.

We cannot trace this evolution fully, though a little of it is indicated to us by the cells in a plasmoidal state in which rigid cell walls have not as yet evolved. Whether protoplasm is still being generated in the sea or not, we cannot tell. If not, then all new living matter is reproduced from the old, but there is no reason to believe that spontaneous generation does not still continue. Experimentation with protoplasm has always been very difficult because of its extremely unstable character. A slight injury to the cell is almost certain to destroy its life so that extended

1 Cf. Snyder, op. cil.; A. C. Lane, The Early Surroundings of Life, in Science, Vol. XXVI, No. 657 (Aug. 2, 1907), pp. I29-143; Joseph McCabe, Evolution, New York, pp. 50-51. Snyder thinks these conditions were much the same as now. McCabe thinks they were very different, being much hotter and with a much heavier atmosphere. 


\section{The Physico-Chemical Basis of Behavior 19}

experiments with it are almost impossible. But biologists may yet succeed in generating life by artificial means, and thus trace fully the course of organic evolution.

This chapter has indicated briefly the characteristics of organic matter which furnish the physico-chemical basis for behavior. 


\section{CHAPTER III}

THE ANATOMICAL AND PHYSIOLOGICAL BASIS OF BEHAVIOR

Metabolism, 20. - Respiration, 20. - Circulation, 20. - Reproduction, 2 I. - Sex, 22. - Theories of the origin of species,23. - Orthogenesis, 24. - Nägeli, 25. - Eimer, 27.-Differentiation of species through the action of external forces, 30. - Lamarck, 30. - Adaptation through the transmission of acquired characters, 30. - Darwin, 32. - Variation, 32. - Selection, 33. - Criticisms of Darwinism, 35.-Weismann, 38. - Inheritance through the germ cell, 38. - Germinal selection, 40. - Panmixia, 42 .

WE must now study the anatomical and physiological characteristics of the organism in order to understand the structural forms and physiological processes which condition behavior. This can best be done by means of a brief survey of organic evolution showing how these forms and processes have evolved and what forces have been and are at work in the animal world.

\section{Physiological Processes}

Metabolism is the fundamental process which goes on in living matter. There are certain other processes which characterize all living matter. Respiration is the process by which all effete matter and other matter not needed by the organism is oxidized and prepared for excretion. Circulation is the process by which water flows into the organism, carrying with it nutritive ingredients, and flows out of the organism, carrying with it the matter to be ex- 


\section{The Anatomical and Physiological Basis 21}

creted. All living matter is characterized by sensitiveness or irritability, which makes it responsive to external influences. I shall discuss this characteristic of living matter later in this book when dealing with the simplest reactions of organisms. As the organism evolves, it acquires a more and more definite form, and then a skeleton which fixes the character of the species and makes it more or less permanent.

The assimilative capacity of the cell is limited. That is to say, when the cell attains a certain size its surface cannot adequately supply it with food because its bulk increases more rapidly than its surface. Furthermore, it becomes too bulky for the strength of the cell walls and breaks into two parts. This phenomenon marks the beginning of reproduction, for these two parts grow and become full-grown cells. I shall now discuss reproduction briefly from two points of view: on the one hand, as to the extent to which traits persist in reproduction; on the other hand, as to the extent to which variation takes place in reproduction. Both of these factors are of great importance in the determination of behavior.

\section{REPRODUCTION}

The lowest type of reproduction has been mentioned above. When a unicellular organism becomes too bulky for the strength of its cell walls, and its volume is too great to be adequately supplied with food by its surface, so that its limit of growth has been attained, it separates into two parts, each of which becomes an organism. This is called reproduction by fission, and the division of the cell into two parts, which is characteristic of all cells, is called mitosis. 
The two new cells become more or less exact copies of the parent cell. Two other forms of reproduction which characterize certain unicellular and some of the lower multicellular species are reproduction by budding and sporulation. Both of these modes of reproduction are rare. In reproduction by budding, new cells grow out of the parent cell, thus forming a multicellular organism. Later the cells break off to become independent organisms, so that in reproduction by budding we have a modified form of the division which takes place in reproduction by fission. In sporulation the parent cell breaks up into several parts, each of which becomes a new cell.

In all forms of reproduction the reproductive power always resides apparently in the nucleus of the parent cell, a part of which goes into the formation of new cells. The characteristics of the parent are transmitted by means of the nucleus to its offspring. However, the cytoplasm of the cell which surrounds the nucleus probably has some influence upon inheritance, though how much is not yet known. Thus the nuclein or chemical compound called chromatin, out of which the nucleus is formed, seems to have the power of forcing the characteristics of the parent upon the offspring. ${ }^{1}$

So far $I$ have been speaking of reproduction in species where the cells are all of the same kind. But this is true only of the lowest species. As we go up in the organic scale we find a specialization among the cells taking place. Certain cells called the germ cells become specialized for reproductive purposes alone, while the other cells or somatic

${ }^{1}$ Cf. Jacques Loeb, in his essay Experimental Study of the Influence of Environment on Animals, in Darwin and Modern Science, edited by A. C. Seward, Cambridge, 1909, p. 247. 


\section{The Anatomical and Physiological Basis 23}

cells no longer have any reproductive function, but perform other functions in the individual organism. Furthermore, there took place later on in organic evolution a specialization among the germ cells themselves into ova or egg cells, and spermatozoa, or sperm cells. In all of the species in which the distinction of sex has appeared, reproduction cannot as a rule take place from a single germ cell as in the asexual species, but the ovum, or egg cell, has to be impregnated or fertilized by the spermatozoon, or sperm cell, before reproduction can take place. Furthermore, there appears among the individual members of these species the distinction of sex. The species is divided into females, who bear the egg cells, and males, who bear sperm cells, while in many other respects as well the two sexes differ greatly from each other.

\section{ORIGIN OF SpECIES}

I have now reviewed briefly the origin and nature of life in general and also in individual organisms. The second great biological problem is that of the origin of species. This problem also is of great importance for the study of behavior, for each species is characterized by specific structural forms and physiological processes which determine specific forms of behavior. It is hardly necessary to offer any proof as to the great diversity of species, since every individual has a personal knowledge of a large number of them. The first great division among organic species is that between animals and plants, while each of these classes may be divided into unicellular and multicellular groups. Animals and plants are therefore classified as protozoa and protophyta, or unicellular animals and plants, and 
metazoa and metaphyta, or multicellular animals and plants. Among these species there is the greatest diversity of form, as indicated among animals by the differences between the minute insects and the huge mammalian forms, such as the elephant, and among the plants by the differences between the algæ and the huge trees. The question as to how this great diversity of organic forms came into existence has always aroused a great deal of interest, and many theories have been propounded to explain it. It was at one time the popular belief that each species is a special creation of divine power, but more recently the attempt has been made to solve the problem of the origin of species in a scientific fashion on the basis of natural forces. I shall discuss these scientific theories under the following classification which, I believe, reveals best the peculiar characteristic of each group of theories. The first class explains the origin of species through orthogenesis; the second class explains it in the main by the action of external forces upon the individual organism; the third class explains it by heterogenesis.

\section{ORTHOGENESIS}

By orthogenesis it is meant that the organism has within it forces which carry it along a certain line of evolution independent of the external forces which play upon it. These internal forces arise in the first place out of the physical and chemical properties of the elements of which the organism is composed. It goes without saying that the first forces in organic evolution are the purely physical and chemical forces inherent in the elements which compose organic matter joined with the external molar forces which bring these elements together. Certainly no scien- 


\section{The Anatomical and Physiological Basis}

tist to-day ought to believe in a supernatural vitalistic force as originating organic evolution. As has already been indicated, the forces which bring into being the organic cell may be similar to those which cause crystallization in inorganic matter. The cell is similar to the crystal in its symmetry and definiteness of form, but is somewhat more complex. The forces which cause the crystal and the cell may in the main be called orthogenetic in the sense that they arise principally out of the inherent characteristics. of the component parts of these organic forms. In the second place, as the organism grows more complex it develops forces peculiar to itself which force it along a more or less definite line of evolution. Thus orthogenetic changes may take place in the cell as a result of the interaction of the parts of the cell, so that an evolution of the organism takes place independent of external forces. All orthogenetic theories attempt to explain organic evolution and the origin of the different species by the workings of these internal forces. It goes without saying, however, that the orthogenetic theories postulate no supernatural or vitalistic force to account for organic evolution, but utilize only purely natural mechanical forces.

Perhaps the most extreme example of an orthogenetic theory is that of Nägeli. He accounted for the earliest formations of organic matter by the purely physical and chemical laws which govern molecular matter, the process being similar to that of crystallization. Then as the organism developed he believed that its evolution was directed in a definite course by what he unfortunately called a completing or perfecting principle ("Vervollkommungsprincip"). Some have thought that he meant by this a 
mystical vitalistic force, but the title of his principal work ${ }^{1}$ indicates that he regarded it as a purely mechanical force. He believed that the existing species have evolved as a result of a phyletic force independent of any external forces, so that the existing species would have been the same had there been no selective forces, and all that selection has done has been to suppress certain species. There is, however, no evidence of any such phyletic force independent of the forces inherent in the individual organism. As we shall see, the continuity of the germ cells is the only thing which is at all similar to a phyletic force. But this also is very different, because the evolution of the germ cells is not directed definitely towards the formation of a distinct species.

Nägeli was forced to take note of the adaptations which characterize all organic forms. He was, however, unwilling to admit that these adaptations were permanent characters of a species. Adaptations might arise as a result of changes in environment and be transmitted by inheritance. But he contended that when the organism returned to its original environment it would soon lose its adaptive characters and would return to its original form which had been given to it by the phyletic force. This he illustrated in the case of certain plants which had originated at a low altitude and had then been transplanted to a high altitude. In the new environment in the course of several generations they acquired some new characters. But after being taken back to their original environment they lost their acquired char-

1 Carl von Nägeli, Mechanisch-Physiologische Theorie der Abstammungslehre, Munich and Leipsic, 1884; A Mechanico-Physiological Theory of Organic Evolution, Chicago, 1898 . 


\section{The Anatomical and Physiological Basis 27}

acters and returned to their original form. It is evident that in this case Nägeli is recognizing the influence of external forces and, furthermore, believed in the transmission of acquired characters which, as we shall see, is very doubtful.

This, however, shows that no theory of organic evolution can be purely orthogenetic, for however much the author of it may strive to account for organic evolution by a purely internal phyletic force, he is forced at some point to recognize the influence of external forces.

This is especially true in the case of Eimer, ${ }^{1}$ who formulated an orthogenetic theory which is, next to that of Nägeli, the best known. Eimer made extensive studies of the coloring of butterflies, and believed that he found in the progressive development of symmetrical color markings evidence of determinate evolution along orthogenetic lines. He thought he distinguished three main forces in organic evolution. The first he called genepistasis, or cessation of development. He believed that certain individuals in a species cease to develop at a certain point in their life history, while other individuals develop further. Thus arise individual differences which in course of time mark the distinction between two species. A different form of cessation of development he called heterepistasis. In this case certain individuals cease to develop in certain parts sooner than the rest of the species, and this gives rise to a new species. The second great force he called halmatogenesis, or saltatory variation. These sudden changes are,

1 Th. Eimer, Die Entstehung der Arten auf Grund von Vererbung erworbener Eigenschaften nach den Gesettzen organischen Wachsens, Jena, I888; On Orthogenesis and the Impotence of Natural Selection in Species-Formation, Chicago, 1898. 
as he described them, similar to the mutations of De Vries which will be discussed later. The third great force was kyesamechania, or hindrance to fertilization. Such hindrance might arise in several ways according to the nature of the species. If in a species fertilization comes about through direct contact of the external sexual organs, fertilization might be prevented by a disproportion in the size of these organs. In other cases fertilization might be prevented by the inability of the sperm to penetrate the ovum either because the outer membrane of the ovum is too thick, or the sperm is too small and too weak to effect penetration. In still other cases the sperm might succeed in penetrating the ovum, but fertilization would not take place because of physico-chemical changes which had arisen either in the sperm or in the ovum which would cause sterility between the two. We shall see that Eimer's kyesamechania, or hindrance to fertilization, as a force in organic evolution was recognized by Romanes under the name of physiological selection.

While these forces in organic evolution described by Eimer are in appearance internal, yet in trying to account for their origin Eimer gave great weight to external influences. He discussed at great length the influence of temperature, nutrition, etc., in causing cessation of development, saltatory changes, and hindrance to fertilization. He has indeed been called a Neo-Lamarckian and illustrates very well how impossible it is for an orthogenesist to ignore external influences in organic evolution. His theory is orthogenetic mainly in the sense that he denied any influence in evolution to indeterminate fluctuating variations and insisted that evolution is determinate. 


\section{The Anatomical and Physiological Basis 29}

In every orthogenetic theory variations which have evolutionary value are called determinate in the sense that these variations are directed along one line towards the development of a distinct species form. In this respect orthogenetic theories vary, for example, from theories based upon selective forces at work upon variations fluctuating in all directions, as, for example, the Darwinian theory. It is, however, unfortunate that these variations are called determinate, for this word suggests a conscious directing power back of organic evolution, and, as has already been noted, none of the modern scientific orthogenesists postulate the existence of any such power. It would be well if the words "determinate" and "indeterminate" used in characterizing variation and evolution could be abolished from biological literature, carrying with them the last vestige of a suggestion of a teleological force in organic evolution.

All orthogenetic theories contain a measure of truth. As we have already seen, the generating of the primordial protoplasm, similar to crystallization, takes place in large part as a result of the molecular forces inherent in the elements which constitute organic matter. The living cell, however, has much greater power than the crystal, for its nuclein acts as a ferment or enzyme for its own synthesis and synthesizes other nuclein of its own kind for new cells, thus determining the continuity of the species. As a rigid anatomical structure develops, the line of evolution becomes more or less narrowly limited and the organism is less amenable to external influences. Furthermore, symmetry and the correlation of parts have much to do with determining the evolution of a species, as we shall see later on. But while organic evolution may be orthogenetic in all 
these respects, it would be a denial of the fundamental law of the conservation of energy to contend that external influences have nothing to do with organic evolution. Furthermore, the effect of external forces is to be seen in the extent to which all organic forms are adapted to their environment, and orthogenesis can never explain these adaptations, for it is inconceivable that all of them could be purely accidental and that there is no causal relation between the environment and these adaptations. We shall therefore now turn to the theories which try to explain the origin of adaptations by giving great weight to external influences in organic evolution.

\section{LAMARCK}

The first complete theory of organic evolution was that of the great French zoölogist Lamarck. ${ }^{1}$ Lamarck was much impressed by the extent to which organic forms are adapted to their environment. He noted that adaptations acquired during the life of the individual usually result from use. For example, a muscle increases in size and in strength as the result of use. A portion of the skin which is frequently used in contact thickens. It occurred to him that the adaptations born in the individual result from the inheritance of characters acquired by his ancestors through use. Thus he explained the long neck of the giraffe by the habit of its short-necked ancestor of stretching its neck in order to eat the foliage on trees. He thought that the slight increase in the length of the neck resulting from this habit was transmitted, and the cumulative result of many such transmissions is the present long neck of the giraffe.

1 J. B. Lamarck, Philosophie zoologique, Paris, 1800. 


\section{The Anatomical and Physiological Basis 31}

According to the same principle, the duck and goose have acquired their web feet as a result of the habit of their ancestors of stretching the skin between the toes when striking the surface of the water and birds have acquired their long toes as a result of the habit of grasping the limbs of trees.

In similar fashion Lamarck explained the degeneration and disappearance of characters by disuse. He contended that if characters are acquired as the result of use, it is logical to assume that they will degenerate and disappear when they fall into disuse. Thus fish living in dark caves become blind because they no longer have any use for their eyes.

Lamarck's explanation of the adaptation of a species to its environment through the transmission of characters acquired through use and of the degeneration and disappearance of these characters through disuse is a very plausible one and has therefore had a very wide currency. But Lamarck never offered a satisfactory explanation of how this transmission of acquired characters actually takes place. In order to furnish scientific proof of this theory, it would be necessary to describe the mechanism by. which the characters acquired by the parent are transmitted to the offspring. Since no such mechanism was described by Lamarck, and since no well-authenticated case of the transmission of an acquired character has been observed, the Lamarckian theory of organic evolution is now almost entirely discredited by scientists. As we shall see, the mechanism by which an organism inherits its traits is of such a nature that the traits acquired by its parents could hardly have any direct effect upon its inheritance. It is evident that 
theories of the origin of species through external forces must after all give great weight to internal forces, for the organism must respond to these external forces and they have their effect upon the organism through the internal organic mechanism. But, on the other hand, we shall see that the characters acquired by individuals may indirectly have a great influence upon the evolution of the species.

\section{DARWIN}

The next great theory of organic evolution based in the main upon the influence of external forces upon the organism is that of Darwin. This theory coming as it did at an opportune moment has been the most famous of all theories of organic evolution. Darwin, like Lamarck, was much impressed with the extent to which organic forms are adapted to their environment. It was customary at that time to explain such adaptations teleologically as being the purposive acts of a divine power. For example, Paley in his famous treatise had likened the universe to a watch in which is revealed the design of its maker. But Darwin repudiated all such teleological explanations of adaptation and attempted to explain them on purely natural grounds. He had noted on the one hand that it is the tendency of every species to increase in numbers beyond the means of subsistence. The question therefore arose in his mind as to what determined which individuals were to survive and which were to perish. He had noted, on the other hand, that there are a great many congenital variations among the members of a species, which variations fluctuate around a central point. These variations are governed, as Quetelet later showed, by the law of probability, and the variations 


\section{The Anatomical and Physiological Basis 33}

are usually no greater than to be included within a normal curve of frequency.

It occurred to Darwin that the individuals who survive in the struggle for existence might be those characterized by variations which would be advantageous to them in this struggle for existence. If, therefore, the individuals with advantageous variations are constantly being selected for survival while the other individuals are perishing, and these variations are being transmitted, a species would gradually be characterized by the variations which have proved to be useful in its struggle for existence. The central thought, then, in the Darwinian theory, as indicated in the title of his great work, ${ }^{1}$ is that of selection which is constantly at work among fluctuating individual variations, preserving those which are advantageous and resulting in their transmission. Darwin and his followers have studied in great detail this force of selection and have distinguished many kinds of selection.

\section{SELECTION}

For example, Darwin believed that the so-called secondary sexual characters, these being the characters which distinguish the sexes from each other, apart from the sexual organs which are the primary sexual characters, are the result of sexual selection. That is to say, the sexes in a species have been governed in their choice of mates by these characters. When a variation has appeared which has proved to be attractive to the opposite sex, it has been selected for survival by the advantage which that individual

1 Charles Darwin, The Origin of Species by Means of Natural Selection or the Preseroation of Favoured Races in the Struggle for Life, London, I859. 
had in mating. Darwin's critics, however, have contended that sexual selection cannot explain most of these secondary sexual characters, because it assumes æsthetic faculties much more highly developed than are to be found in most species.

Romanes suggested what he called physiological selection, ${ }_{1}$ or hindrance to fertilization, which, as we have seen, had already been described by Eimer under the name of kyesamechania. As a result of such restrictions upon the possibility of fertilization between certain individuals, certain types would be extinguished while others would survive, or two or more distinct types would survive which might in course of time become distinct species.

Geographical selection or isolation as a force in organic evolution and in the formation of species has been discussed by Wagner, Romanes, Gulick, and others. If a portion of a species becomes isolated from the rest of the species by geographical barriers or by any other means, a new species is likely to result from such isolation. If intercrossing is rendered entirely impossible between the two isolated groups, each group will tend to vary along its own lines, and in course of time there may be two species where formerly there was but one. Thomson has described this form of selection in the following words: "This term is used to include all the means which restrict the range of intercrossing within a species: geographical barriers, such as arise when a peninsula becomes an island; temporal barriers, such as arise when the members of a species reach sexual maturity at different times of year; habitudinal barriers, when a species splits into two or more castes with different habits of life; physiological barriers, such as arise by some variation in

1 G. J. Romanes, Journal of the Linnean Society, Vol. XIX, pp. 337-441. 
the reproductive organs; and psychological barriers, which rest on profound antipathies." 1

Reproductive or genetic selection is a form of selection suggested by Karl Pearson. If the highest fertility in a species becomes correlated with certain characters, variations in those characters will have the advantage, because they will be reproduced most frequently. Thus those variations are most likely to persist and become characteristic of the species.

It is impossible in this brief review to discuss at greater length the subject of selection, to which a great deal of study has been given. I shall now take up the principal criticisms of the Darwinian theory, which are of great importance because they have stimulated much of the recent work in biology.

\section{Criticisms OF DARWINISM}

The first main criticism of Darwinism as a synthetic theory of organic evolution is that it gives no adequate explanation of the origin of individual variations. It is true that Darwin offered his theory of pangenesis ${ }^{2}$ as a tentative explanation of the origin of variations. But Darwin himself was very uncertain as to the soundness of this theory, and since his time no evidence has been found in support of it. According to this theory the germ cells are formed by small particles, which Darwin called gemmules, which are thrown off by the somatic cells in all parts of the organism. Now if any new characters have been acquired by the somatic cells, they will be reflected in changes

1 J. A. Thomson, Darwinism and Human Life, New York, rgog, pp. 210-11.

2 Darwin, The Variation of Animals and Plants under Domestication, London, 1868. 
in these gemmules which compose the germ cells. Thus acquired characters will cause changes in the germ cells and variations in the offspring. So that Darwin's pangenetic theory is Lamarckian in that it recognizes the influence of acquired characters in heredity. He went further than Lamarck, however, in suggesting definitely by what mechanism this transmission of acquired characters takes place. It is curious that Darwin, who had repudiated Lamarckism elsewhere, should have recognized it in his theory of pangenesis. Beyond presenting this theory, Darwin had no explanation to offer as to the origin of variations.

The next great criticism of the Darwinian theory was that variations are not at their origin usually of sufficient size to be advantageous or disadvantageous in the struggle for existence. The fortuitous, fluctuating variations which, according to the Darwinian theory, furnish the material for natural selection are usually so insignificant that they cannot have a life-or-death value in the struggle for existence of the individuals characterized by them. For example, the polar bear whose white fur is undoubtedly advantageous to it has in all probability descended from the brown bear. But if it has acquired its white coat through the selection of favorable fluctuating variations, it is difficult to see how the first white hairs or small patches of white which appeared in the fur of certain of the brown bears could have had sufficient selective value to give these individuals the advantage over their fellows. It is evident that a variation, even though it is in a favorable direction, has no advantage over other variations unless it has immediate selective value, for otherwise it will be swamped by interbreeding. A variation must therefore be of suff- 


\section{The Anatomical and Physiological Basis 37}

cient size to be of immediate selective value if it is to be perpetuated. But the critics of Darwinism have contended that most of the fluctuating variations are not of sufficient size to have selective value, and therefore selection could not have been the main force in the origin of species and in organic evolution.

Another criticism which has been made of the Darwinian theory is that there has not been enough geological time for organic evolution according to Darwinian methods. It is evident that the selection of slight variations is a slow process, and that it would take a vast number of such variations to build up the complex organic forms now in existence. It was estimated by Kelvin, Tait, and others by means of deductions made from the central heat of the earth, from the rate of deposition of the calcareous deposits, from the increase in the amount of salt in the sea, and from various other sources, that the surface of the earth has been inhabitable for a period under one hundred million years. Some of the Darwinian evolutionists have estimated that it must have taken thousands of millions of years for organic evolution by the selection of slight variations. If, therefore, the estimates of these physicists are correct, there has not been sufficient time for Darwinian evolution. But the recent discovery of radium has revealed sources of heat in the sun which were not conceived of before. This indicates that the life of the earth may have been very much longer than was estimated by Kelvin and Tait, and that there may have been enough time for Darwinian evolution.

It is needless to emphasize here the vast influence which the Darwinian theory has had. As has already been noted, Darwin was determined to account for the origin of species 
and organic evolution on natural grounds, and with painstaking care he accumulated a vast amount of data to support his brilliant generalizations. His theory came with convincing power to his own generation and proved once for all the fact of organic evolution, while it also threw a great deal of light upon the manner in which this evolution must have taken place. But, as we have seen, serious criticisms have been made of the Darwinian theory, and we must discuss the theories which are trying to supplant the Darwinian theory. Before doing so I wish to discuss the theories of the great Neo-Darwinian biologist, Weismann, ${ }^{1}$ who, though he has varied in several important respects from Darwin, has tried to answer the criticisms of Darwin and to prove the validity of the central Darwinian doctrine.

\section{WEISMANN}

The first great accomplishment of Weismann was that he completely refuted the Lamarckian theory. In this respect he varied from Darwin, since, as we have seen, Darwin was not entirely free from Lamarckism. By means of a painstaking analysis of many of the supposed cases of Lamarckian inheritance, Weismann showed that the hereditary transmission of characters acquired through use and disuse is not possible. On the contrary, he showed that inheritance must be through the germ cells, upon which acquired characters can have no direct effect. He therefore devoted much attention to the study of the constitution of the germ cells. He described the constitution of the germ cell as follows. The lowest chemical unit in the germ

1 A. Weismann, The Germ Plasm, London, 1893; On Germinal Selection as a Source of Definite Variation, Chicago, 1896. 


\section{The Anatomical and Physiological Basis}

cell as elsewhere is the molecule. The lowest biological unit is, however, more complex and is composed of a number of molecules. This biological unit Weismann called biophor. The biophor is a true form of living matter in that it absorbs nutriment during its life. These biophors are organized into determinants, each of which represents a special kind of somatic cell. The determinants are in turn organized into $i d s$, each of which represents a distinct part of the organism. Assuming as he did that inheritance is entirely through the germ cells, it was incumbent upon Weismann to describe the mechanism by which the germ cell determines the character of the fully developed organism. He was not the first who discussed the structure of the germ cell, for various other biologists have set forth hypotheses as to this question. We find several of them postulating ultimate biological units like Weismann's biophors. Thus we have Darwin with his gemmules, Spencer with his physiological units, Nägeli with his micellæ, Galton with his stirps, De Vries with his pangenes, Haeckel with his plastidules, etc. All these so-called micromeric theories assume that each character in the fully developed organism can be traced back to a unit in the germ cell. There have been other theories as to the constitution of the germ cell and the mechanism of inheritance, such as the biogen theory of Verworn, the physical machine theory of Delage, the chemism theory of Le Dantec, but I have no space to discuss these theories here. It has so far been impossible to furnish any positive proof of any of these theories because of the microscopic and unstable nature of the germ cell. It is, however, necessary for each writer on the subject to adopt some sort of an hypothesis as to the nature 
of the germ cell, however impossible it may be to prove or disprove this hypothesis.

Having assumed an hypothesis as to the nature of the germ cell, Weismann now turned his attention to answering the critics of Darwinism. He was forced to admit that many favorable variations are not at first of sufficient size to possess a life-or-death significance in the struggle for existence. In order, therefore, to explain how slight favorable variations are preserved, he set forth his theory of germinal selection. This theory extends the idea of selection to the germ cell and assumes a selective process among the parts of the cell as well as among complete organisms. We have already noted that Weismann believed that the units within a cell absorb nutriment as well as the cell as a whole. There may therefore arise among these units a struggle for nutriment. If one of these units happens to be a little stronger than the others, it will secure a larger share of the nutriment and will therefore grow to a larger size. Its increase in size will give it still further advantage in the struggle for nutriment, and will result in a further increase in size. This advantage secured by one determinant over others would be perpetuated from generation to generation of germ cells, and thus variation in a definite direction would come about. Such definite variations would in the case of some determinants be in the direction of degeneration and decrease in size where the determinant is at a disadvantage in the struggle for nutriment. These variations in a definite direction in determinants would be manifested in definitely directed variations in the fully developed organism. But these variations might for a long time not be of sufficient size to have selective value. As soon, however, as the 
variation had become of sufficient size to have life-ordeath significance, external forces would immediately exert a selective power over them, preserving and perpetuating the individuals manifesting favorable variations and suppressing those with unfavorable variations.

Weismann believed that by means of his theory of germinal selection he was able to show how variations are furnished as material for natural selection. But while he has extended the idea of a struggle and of selection to the germ cell, it is evident that this struggle and selection are very different from the Darwinian struggle for existence and natural selection. The Weismannian theory differs very greatly from the Darwinian in that the materials for selection are no longer slight fluctuating variations, but variations of considerable size directed in a definite line of development. Thus the theory of germinal selection is more nearly a causo-mechanical explanation of orthogenetic variation, that is to say, variation directed along a definite line by internal forces, than it is a theory of variations caused by external forces. The theory of germinal selection may be true, but it furnishes no assistance to the Darwinian theory of natural selection.

Another criticism of the Darwinian theory has been that it fails to account for the degeneration of useless parts. If a useless part is harmful to the organism and hinders it in the struggle for existence, it will be eliminated by natural selection. But if the useless part is harmless and does not hinder the individual in the struggle for existence, there seems to be no reason why it should not persist in its full vigor as much as the useful parts. Weismann endeavored to explain the degeneration of useless parts by means of his the- 
ory of panmixia. As we have seen, variations come about as a result of the success or failure of the determinants to secure nutriment. When a variation has become of suffcient size to be of life-or death significance, if it is advantageous it will be preserved and encouraged by natural selection, if it is disadvantageous it will be suppressed in a similar fashion. A variation may, however, be neither advantageous nor disadvantageous, or after having been advantageous, it may have become useless. In this case it will no longer have the force of natural selection in its support. It will therefore persist only so long as its determinant is successful in securing nutriment. When its determinant happens to lose in this struggle, a definitely directed downward variation will commence which will not be checked by natural selection, and thus the part will degenerate and may in course of time disappear entirely. Thus, according to Weismann's theory of panmixia, in the mixing of parts which takes place at reproduction the determinants which represent the parts which are advantageous to the organism, and are therefore being supported by natural selection, will have the advantage in the struggle for nutriment within the germ cell, while the determinants which represent useless parts will lack the support of natural selection and are certain in course of time to lose ground in the struggle for nutriment and to degenerate. The theory of panmixia is, however, as hypothetical as the theory of germinal selection and the nature of the germ cell as described by Weismann, so that it is impossible to say how true an explanation it is of the degeneration of useless parts. Certain it is that many such useless parts are to be found in the vestigial and rudimentary organs which exist in all 


\section{The Anatomical and Physiological Basis 43}

organic forms, and for which an explanation must be given in any complete theory of organic evolution.

Weismann devoted a good deal of attention to the explanation of the origin of variations. We have already seen in the theory of germinal selection how variations may come about as the result of changes in the nutriment of the determinants. Weismann believed furthermore that amphimixis, or reproduction by two parents, is of great value in stimulating variability. In amphimixis two lines of descent are crossed, and in the mixing of determinants which takes place there is much greater possibility of new combinations of characters. Some attempt has been made to determine whether there is more variability in amphimixis than in self-fertilization or parthenogenesis. Nothing conclusive has been learned, though there is some indication that there are no more slight fluctuating variations in amphimixis than in other forms of reproduction. But this does not mean necessarily that amphimixis does not cause more of the large non-fluctuating variations.

Weismann has insisted that he has proved that variation is guided by utility, and that the degree of adaptiveness of a part evokes the direction of variation of that part. He has furthermore insisted that he has proved that there is no conscious directing power in adaptations, however purposive they may seem. We have seen that he may not be correct in thinking that all variations are guided by utility, inasmuch as some of them may be orthogenetic in their origin and therefore independent of considerations of utility. But there can be little doubt that Weismann, next to Darwin, has done more than any one else to show that 
organic evolution has not in the slightest degree been teleological, but has been the result of purely natural forces. Furthermore, Weismann has stimulated greatly the study of the internal forces in organic evolution by insisting that the germ cell is the sole agent of heredity. 


\section{CHAPTER IV}

THE ANATOMICAL AND PHYSIOLOGICAL BASIS OF BEHAVIOR (Continued)

Heterogenesis, 45. - Discontinuous variations, 46. - Bateson, 46. - Substantive and meristic variations, 46. - Symmetry, 47.-De Vries, 5I. - The mutation theory, 5I. - Micromeric theories of the germ cell, 53. - Unit characters, 55. - Mendel, 55. - The Mendelian law of inheritance, 55.- Dominant and recessive characters, 55. - The segregation of allelomorphic characters, 56. - Homozygotes and heterozygotes, 56. - The Mendelian explanation of particulate, exclusive, and blended inheritance, 57. - Reversion or atavism, 59. - Galton's laws of ancestral inheritance and of filial regression, 60. - Mendelian criticism of Galton's laws, 6o. - Statistical and physiological laws of inheritance, 6o.- The exaggerated claims for Mendelism, 63. - Johannsen's theory of pure lines, 64. - Sex determination, 65. - Sex as a unit character, 66. - The biological basis for psychology, anthropology, and sociology, 68 .

WE have now discussed orthogenesis as represented in the theories of Nägeli and Eimer and theories of organic evolution based in the main upon the action of external forces as represented in Lamarck and Darwin, while in Weismann we find an example of a theory of organic evolution which combines the internal with the external forces. Weismann will therefore serve well as an introduction to heterogenetic theories of organic evolution.

\section{HETEROGENESIS}

By heterogenesis it is meant that organic evolution and the origin of species have resulted from occasional, sudden, 
and fixed variations which may be large or small, and which appear in offspring and form the starting point for new types and species. Inasmuch as the heterogenesists account for these sudden variations or mutations, as they are frequently called, by the interaction of internal orthogenetic forces and external environmental forces, heterogenetic theories form a type which is a sort of combination of the two types of theories which I have been discussing.

Heterogenetic variations are usually called discontinuous in the sense that they do not come gradually as the result of slight changes, but suddenly and all at once. In this respect they are contrasted with the continuous Darwinian variations, which appear as the result of the selection of slight fluctuating variations. Discontinuous variations are not governed by the law of probability like the fluctuating variations and do not fall within a normal curve of frequency. Heterogenesis as a theory of species forming has been advocated by various biologists since the time of Darwin, such as von Kölliker, Galton, Bateson, Emery, Korschinsky, de Vries, etc. Some of these biologists have been anti-Darwinian; others have agreed with the Darwinian theory in the main, but have varied from it in believing that discontinuous variations have had something to do with the formation of species. I wish to speak in particular of the theories of Bateson and of de Vries.

\section{BATESON}

Bateson ${ }^{1}$ classifies variations as falling under two main types, namely, substantive variations and meristic variations. By a substantive variation he means any change

1 W. Bateson, Materials for the Sludy of Variation, London, 1894. 


\section{The Anatomical and Physiological Basis 47}

in the internal composition or structure of any part of the organism. By a meristic variation he means any change in the arrangement or repetition of the parts of the organism. He has enumerated and described a large number of cases of meristic variation. In introducing the description of these variations he has discussed at some length the subject of symmetry in organic forms.

Symmetry is an almost universal phenomenon in the organic world. Three main types of symmetry may be distinguished, - linear, bilateral, and radial symmetry. In linear symmetry parts are repeated in consecutive order, as, for example, in the case of the rings of a worm or snake or of the vertebræ in a vertebrate. In bilateral symmetry a part is repeated once in a corresponding position, as in the case of the arms and the legs of a vertebrate. In radial symmetry parts are repeated, branching out from a central point, as in the tentacles of a starfish or the petals of a flower. Some have reasoned in accordance with the Darwinian theory that symmetrical forms are adaptations resulting from the workings of natural selection. It is true that symmetrical parts do frequently adapt an organism to its environment. For example, freely moving animal forms are usually characterized by linear and bilateral symmetry, though a few freely moving animals are characterized by radial symmetry, as, for example, the sea urchin. Immobile plant forms usually display radial symmetry, though linear symmetry also is frequently found, as in the stalk of a plant, and bilateral symmetry less frequently, as in some forms of flowers. Immobile animal forms also are usually characterized by radial symmetry, as, for example, the sea anemone. But the fact that symme- 
try is frequently advantageous to an organic form does not prove necessarily that it is an adaptation. For example, it has been pointed out that the symmetry is frequently more perfect than is necessary for adaptation, so that there must have been some force at work apart from adaptation in causing this symmetry. Furthermore, symmetry is sometimes disadvantageous to an organic form. These facts indicate that symmetry is probably caused in the main by orthogenetic forces and that, though natural selection may have suppressed many symmetrical forms which have proved to be disadvantageous and has preserved many other symmetrical forms which have proved to be advantageous, it has had nothing to do with causing this symmetry.

I have already called attention to the fact that crystals are symmetrical like organic forms. It is quite possible that such symmetry as exists in the primordial protoplasm has been caused in a fashion similar to the symmetry of crystals; namely, by the forces inherent in the molecules of the elements which composed it. But this would not be a sufficient explanation for the symmetry which characterizes more complex organic forms. The symmetry of these complex forms must in all probability be traced back to the mitosis or division of the cell. All organic growth takes place as the result of such mitosis. A cell usually divides into equal parts, and the nuclein which is divided between the two new parts carries the same characters, so that it is not surprising that there should be the repetition of parts which, as we have seen, characterizes symmetry. It has been observed in certain bilateral organisms that the first cell division marks the dividing line between the two 


\section{The Anatomical and Physiological Basis}

corresponding sides of the organisms. The complete explanation of symmetry would in all probability be much more complicated than has been indicated, but such a full explanation has not yet been furnished us by the biologists, and I have not the space to discuss it further here. What has been said is sufficient to indicate how symmetry may have resulted orthogenetically from the internal structure and forces of the organism.

Bateson, who is opposed to Darwinism in certain important respects, believes that symmetry is orthogenetic in its origin, though he offers no explanation for the development of symmetrical forms. Many of the discontinuous meristic variations which he has described are asymmetries or variations from the usual symmetrical forms. In another place he has suggested that heredity is a special case of the symmetrical division of the cell, while genetic variation is a consequence of asymmetrical division of the cell. As Bateson himself says, we know so little as yet with regard to mitosis that it is impossible to judge as to the validity of this hypothesis. Assuming, however, that these variations do result from the asymmetrical divisions of the cell, the important question is as to whether these variations are perpetuated and become the permanent characters of a type. Such discontinuous variations have been observed for a very long time and have been called sports, monstrosities, teratological forms, etc. They have usually been regarded as abnormalities which could not persist permanently, though sometimes reappearing through several generations. It has been contended that discontinuous variations are usually repetitions or misplacements of old parts, and not appearances of new structures; that they are 
very rare, and are not usually inherited because they are swamped by intercrossing and so cannot account for the discontinuity of species. Bateson, however, believes that these discontinuous variations are inherited and may form the characters of a new type, though I suppose he would not deny that if these new characters were sufficiently disadvantageous to the organism they might be eliminated by natural selection. If his suggestion that discontinuous variations result from asymmetrical cell divisions is true, and that these discontinuous variations are inheritable, then he must believe that these variations are normally transmitted in symmetrical cell divisions. It would seem, then, as if the organism would in course of time lose all symmetry if many of these variations took place. It may be, however, that not all of these asymmetrical cell divisions result in asymmetrical variations in the fully developed organism, while the organisms which become most asymmetrical are eliminated by natural selection, because of their unfitness for their environment.

At this point should be noted the part played by the correlation of parts as a cause of variation. Many of the parts of an organism are so related to each other that a change in one of them necessitates changes in the other. - This may be because the parts are repetitions of each other as in symmetry, or because they grow out of and after each other. Thus a single variation at the origin of the individual organism may result in a more or less complicated series of changes in the fully developed organism. 


\section{The Anatomical and Physiological Basis 51}

\section{DE VRIES}

Let us now turn to de Vries, ${ }^{1}$ who is perhaps the most distinguished representative of the theory of the origin of species by discontinuous variations or mutations, as he usually calls them. De Vries has carried on many painstaking experiments with plants in order to study these mutations. He has been most successful with the great evening primrose, or Enothera Lamarckiana. This plant, which he believes came from America, he found growing in a wild state near his home in Amsterdam apparently in a state of mutability. He experimented with this plant through several generations and secured seven varying forms which he believes are new mutations. These new forms bred true when self-fertilized, and de Vries believes that they are elementary species. He has formulated the theory that an organism may pass through a state of mutability when one or more of these mutations will appear. This idea had already been suggested by Galton under the name of organic instability, and had been aptly illustrated by him by means of a polyhedron, which is quite stable on one of its bases, but very unstable on another. Such instability or mutability may be caused by a change in environment. Or it may result from the cumulative effect of forces in the environment which are causing slight internal changes which suddenly manifest themselves in one or more external changes in the organism. Just how these environmental forces affect the germ cells in such a fashion as to cause these mutations has not been clearly shown by any of the mutationists. Their theory sounds a little like Neo-

${ }^{1}$ H. de Vries, Die Mutationstheorie, Leipsic, Igor; Species and Varieties, their Origin by Mutation, Chicago, Ig05. 
Lamarckism because of the emphasis which is laid upon the direct effect of the environment upon inheritance. But I believe they all repudiate Lamarckism, and they may yet succeed in showing how the environment can have this direct effect upon heredity.

Unfortunately for de Vries, it has been said that the plant with which he experimented, the Enothera Lamarckiana, was a hybrid which had come from the Jardin des Plantes in Paris. If this is the case, these varying forms which he believed to be mutants may be ancestral forms reappearing in these descendants. Until this question is decided it will be impossible to estimate at its true value the work of de Vries. With regard to the mutation theory in general there have been as yet few if any well-authenticated cases of such mutations, for it is not always possible to tell whether or not these apparent mutations are the result of hybridization.

If the mutation theory could be proved, it would avoid several of the difficulties in the natural selection theory of the formation of species. Morgan has summed up the advantages of the mutation theory as follows: -

"I. Since the mutations appear fully formed from the beginning, there is no difficulty in accounting for the incipient stages in the development of an organ, and since the organ may persist, even when it has no value to the race, it may become further developed by later mutations and may come to have finally an important relation to the life of the individual.

" 2 . The new mutations may appear in large numbers, and of the different kinds those will persist that can get the foothold. On account of the large number of times that the same mutations appear, the danger of becoming swamped through crossing with the original form will be lessened in proportion to the number of new individuals that arise.

" 3 . If the time of reaching maturity in the new form is different 


\section{The Anatomical and Physiological Basis}

from that in the parent forms, then the new species will be kept from crossing with the parent form, and since this new character will be present from the beginning, the new form will have much better chances of surviving than if a difference in time of reaching maturity had to be gradually acquired.

"4. The new species that appear may be in some cases already adapted to live, in a different environment from that occupied by the parent form; and if so, it will be isolated from the beginning, which will be an advantage in avoiding the bad effects of intercrossing.

"5. It is well known that the differences between related species consist largely in differences of unimportant organs, and this is in harmony with the mutation theory, but one of the real difficulties of the selection theory.

"6. Useless or even slightly injurious characters may appear as mutations, and if they do not seriously affect the perpetuation of the race, they may persist." 1

\section{Micromeric Theories}

It must be evident from what has gone before how important it is to study the internal structure of the germ cell. If it be true, as Weismann and other great biologists have contended, that all inherited characters are transmitted through the germ cell and that all genetic variations arise in the germ cell, it is evident that we cannot hope to know much more about heredity and variation until we know more as to the nature of the germ cell. As has been noted, it is difficult to learn anything definite about the germ cell because of its miscroscopic size and unstable nature. And yet it is necessary for each writer on the subject to adopt some sort of an hypothesis as to the nature of the germ cell. Consequently there are numerous micromeric theories, as, for example, the gemmule theory of Darwin and the biophor theory of Weismann, in which it is assumed that

${ }^{1}$ T. H. Morgan, Evolution and Adaptation, New York, I903, pp. 298-299. 
each character in the organism can be traced back to a unit in the germ cell. By extirpating certain chromosomes in the germ cell and then noting how the organism which develops from it varies from the normal organism, it might be possible to determine whether specific characters are determined by specific units in the germ cell. This would furnish a test of micromeric theories. But it is doubtful if this experiment could be carried out successfully, partly on account of the minute size of the germ cell, but also because the shock of the operation would probably either kill the cell or at least derange its development very greatly.

There has, however, been some criticism of these micromeric theories. For example, Loeb has said that "morphological structures can only play a rôle through their physical and chemical properties," ultimate causes of the phenomena of heredity and variation are to be found not in any vital unit in the cell, but in the properties of the elements of which the cell is composed. Le Dantec ${ }^{2}$ has criticized much more drastically these micromeric theories. He says that the micromerists, while admitting that they cannot explain one of these units, have only complicated the problem by assuming a vast number of them. In the place of these micromeric theories he proposes his chemism theory. According to this theory a species is distinguished by the chemical composition of its germ cells. A chemism theory can take no primary account of form, but as conditions of chemical identity will usually involve identity of form, the individuals composing a chemical species will possess a similar or identical form.

I J. Loeb, The Dynamics of Living Matter, New York, I906, p. I86.

${ }^{2}$ F. Le Dantec, Traité de biologie, Paris, 1906. 


\section{The Anatomical and Physiological Basis 55}

There have been several other physico-chemical theories as to the nature of the mechanism by which the germ cell transmits characters, but I have not the space to discuss them here.

\section{MendeL}

The idea of unit characters, namely, the idea that the characters in an organism may be traced back to distinct units in the germ cell, is now very popular among biologists, probably in large part because of the rediscovery of the work of Mendel. The facts as to this rediscovery in the year I900 are too well known to need repetition here. The discovery immediately aroused great interest, because it seemed to confirm conclusions arrived at by de Vries, Correns, Tschermak, and others. The significant thing about the work of Mendel was that he had devised an experimental method of studying heredity and variation. After crossing certain species, varieties, or types he had traced the characters in which they differed through several generations in order to determine in what proportion and in what manner they reappeared. As a result of these experiments he formulated a law governing the transmission of unit characters in hybridization. Mendel had noted that in most if not all species, varieties, and types there are certain characters which when hybridization takes place do not blend with the corresponding characters in the other species, variety, or type. That is to say, in the hybrid either the one or the other of these alternative characters appears. Such characters have been called, I believe, by Bateson, allelomorphs. Mendel discovered that in some of these allelomorphic pairs of characters one of the characters is usually dominant and the other recessive. 
That is to say, the dominant character appears more frequently in the sum total of the offspring throughout any number of generations than the recessive character.

Furthermore, he discovered what the proportion between the dominant and recessive characters in the offspring usually is. He found that in the firstgeneration all the offspring bear the dominant character. But in the second generation three fourths of the offspring bear the dominant character while one fourth bear the recessive character. The descendants of the individuals bearing the recessive character display thereafter only the recessive character. Apparently, therefore, the dominant character has been eliminated from the inheritance of this line of descent. Of the offspring bearing the dominant character in the second generation, one third give birth thereafter to individuals bearing only the dominant character. Apparently, therefore, the recessive character has been eliminated from the inheritance of this line of descent. The descendants of the other offspring in the second generation bearing the dominant character again break up into individuals bearing the dominant and recessive characters in a proportion of three to one, and the same process takes place of a segregation of lines of descent in which there is transmitted only one of the two characters.

The fertilized germ cells in hybridization from which one of a pair of allelomorphic characters has been eliminated have been named homozygotes, while the fertilized germ cells which bear both of the characters of an allelomorphic pair are called heterozygotes. The segregation of allelomorphic characters in homozygotes has been called by Bateson the most significant part of the Mendelian law. ${ }^{1}$ Bateson

1 W. Bateson, Mendel's Principles of Heredity, Cambridge, 1909, chap. 15. 


\section{The Anatomical and Physiological Basis 57}

believes that such segregation explains purity of type. That is to say, a pure-bred organism is one which is entirely homozygous. If this is true, the Mendelian law is of great value in showing what constitutes purity of type.

It is believed by Mendelians that the Mendelian law explains some kinds of inheritance, while some of them seem to think that it explains all kinds of inheritance. For example, particulate or mosaic inheritance is explained by the Mendelians according to the Mendelian law. In such inheritance an offspring displays the characters of one parent in certain of its parts, while it displays the characters of the other parent in other parts, in such a fashion as to present a sort of mosaic pattern of the characters of the two parents. We have an example of this in the case of piebald coloring, where the offspring displays the color of one parent in certain parts of the body and of the other parent in other parts. The Mendelians believe that the offspring is thus displaying the unit characters of both parents. In the case of piebald coloring it is uncertain whether the appearance of the colors of both parents is due to imperfect dominance or is because the coloring of different parts of the body is represented by different unit characters.

The Mendelians believe that exclusive inheritance also can be explained by the Mendelian law. In this form of inheritance the offspring resembles one parent exclusively. According to the Mendelians such inheritance takes place when one parent bears all the dominant characters; that is to say, is thoroughly prepotent according to the older manner of expressing it.

Some of the Mendelians seem to think that blended inheritance also is explained by the Mendelian law. Such 
inheritance exists when the characters of the two parents are so mingled that neither character appears distinctly. Thus when a white is crossed with a negro there is derived a shade of black which is called mulatto. Karl Pearson ${ }^{1}$ states that he has searched through all the literature on the subject and has found records of only three cases where the crossing of a white and a negro resulted in black or white in the offspring, and has offered further evidence to show that such a crossing almost invariably results in a blend. The same is true of a great many other characters, and it is difficult to understand why certain Mendelians try to apply the Mendelian law to these cases. ${ }^{2}$ It may be because they think that in a case of blended inheritance the allelomorphic characters are equally potent, and therefore both of them manifest themselves in the offspring. But this seems to indicate that unit characters are not as sharply distinguished from each other as the Mendelians would have us believe.

Because of the exaggerated claims of some of the Mendelians, it is well to note carefully just what Mendelism has taught us. In the first place, Mendelism throws no direct light upon the nature of the germ cell, though it may indirectly be of great assistance in criticizing certain of the theories as to the nature of the germ cell. For example, inasmuch as the Mendelian law seems to indicate the existence of unit characters, it gives valuable support to micromeric theories of the nature of the germ cell. Furthermore, Mendelism does not postulate discontinuous variations or reveal their causes. So far as I know, nowhere in his writ-

1 Biometrika, Vol. VI, pp. 348-353 (Nov., I910).

2 C. B. Davenport has made experiments which he thinks prove that the color of the mulatto is not a blend. See $A m$. Naturalist, Vol. xlv (IgIr). 


\section{The Anatomical and Physiological Basis 59}

ings did Mendel express a belief in such discontinuous variations, though they seem to imply such a belief. It has been suggested by Bateson that mutations may be recessives appearing after a crossing, but if this be the case, it would seem as if they were not mutations or discontinuous variations at all, but simply reappearances of ancestral characters. Nothing new, therefore, could arise as the result of the reappearances of these ancestral characters except as new combinations of them constitute new types.

Bateson has also attempted to explain reversion by means of Mendelism. He speaks as follows: "Reversion occurs when the sum total of the factors returns to that which it has been in some original type. Such a return may be brought about by the omission of an element or elements, as when the rose-comb fowl for any reason has a singlecombed offspring. Conversely, the return may occur by the addition of some missing element needed to complete the original type. As yet no means are known by which the omission or addition of elements can be made at will, except by crossing. Reversion on crossing is thus the particular case in which one or more missing factors are brought in by the parents of the cross-bred. The most striking cases of such reversion on crossing are those in which neither parent seems to the observer to contain anything specially reminiscent of the original type, and yet the offspring of the cross are all of that type." 1 It may well be that these cases which result from hybridization and which Darwin called "reversion on crossing" can be explained on Mendelian grounds. But de Vries calls them cases of "false atavism or vicinism" which are due to crossing. He be-

1 Bateson, op. cit., p. 279. 
lieves that most cases of apparent reversion are due to crossing and that "true atavism, or reversion caused by an innate latent tendency, seems to be very rare." 1 The Mendelians have not yet explained the cases of true reversion. It is quite possible that the idea of unit characters is needed to explain reversion, but these unit characters do not act in cases of reversion necessarily in accordance with the Mendelian law.

\section{GaLton}

It has been claimed by Bateson and other Mendelians that Galton's law of ancestral inheritance is nullified by the Mendelian law. Galton's law was to the effect that an individual inherited on the average one fourth of his characters from each parent, one sixteenth from each grandparent, one sixty-fourth from each great grandparent, etc., so that the sum total of these ancestral contributions would amount to unity. The Mendelians have pointed out that, for example, in a case of exclusive inheritance an individual may inherit all his characters from and through one parent so that the other parent will not be represented at all in his inheritance. Thus Galton's law is not a physiological formula which can be applied in every individual case. The Galtonians have, however, asserted that even though it may not be a physiological formula, it is still a statistical formula which applies on the average to a large number of generations breeding freely. But it is contended by Bateson that the discovery of the fact of segregation has destroyed the utility of Galton's law as a statistical formula also. "At the time that Galton's views were promulgated,

${ }^{1}$ De Vries, op. cit., pp. $187-188$. 


\section{The Anatomical and Physiological Basis 61}

nothing was known of segregation. The supposition that any individual, whatever its own characters, was capable of carrying on and transmitting to its posterity any of the characters exhibited by its immediate progenitors, at all events, was generally received without question by biologists. According to that idea the number of classes of individuals differing in respect of their ancestral composition and transmitting powers is to be regarded as indefinitely large, whereas in all cases of sensible allelomorphism the number of classes of individuals is three only, two being homozygous and one heterozygous. The difference between the two schemes is thus absolute and irreconcileable." 1 It may well be that the proportion between the average contributions of the different ancestors is not correct as stated by Galton. As a matter of fact, his leading disciple, Karl Pearson, has proposed a different ratio. But I believe that the law of ancestral inheritance is true to this extent, that the more distant the ancestors, the less the resemblance tends to become between them and their descendants. This is true if for no other reason because the more distant the ancestors, the more time there has been for variations and mutations to arise which will lessen their resemblance to their descendants. It is therefore useless for the Mendelians to deny entirely Galton's law as a statistical formula, however successful may be their criticism of it as a physiological formula. They are, however, right in denying that it is a law of inheritance, for it does not describe how inheritance actually takes place in a single case, but merely indicates some of the results of inheritance in a large number of cases.

1 Bateson, op. cit., p. 127 . 
I do not know whether the Mendelians have attacked Galton's law of filial regression, but it seems as if they would attack this law also on the same grounds upon which they have attacked the law of ancestral inheritance. The law of filial regression as stated by Galton was to the effect that children tend to approximate the mean or average of the stock more closely than their parents. The Mendelians might point out that inasmuch as this is not necessarily true in any individual case, this law is not a physiological formula any more than the law of ancestral inheritance. They might also attack it as a statistical formula on the ground that in cases of segregation there is no regression to the characters of the average of the ancestors. It is evident that Galton's law is simply another way of stating the familiar statistical law that any group of phenomena tend to approximate a normal mean or average, which can be represented graphically by the normal curve of frequency. Thus in any stock which is fairly stable its members will tend to approximate the mean or average of that stock. But it must be noted that in order that there shall be regression towards the mean, there must already have been at least as much digression from it. And in order that there shall be any change in the type or species, there must be at least a little more digression from the mean than there is regression towards it. The law of filial regression is, therefore, not very illuminating and is perhaps even somewhat misleading, inasmuch as it takes no note of the digression from the mean of the stock which must precede and accompany regression towards this mean.

The attitude of the Mendelians towards Galton's law of ancestral inheritance illustrates their tendency to depre- 


\section{The Anatomical and Physiological Basis 63}

ciate greatly the value of statistical methods of studying variation and inheritance in favor of their own experimental methods. It is true that their experimental methods can be used in a great many places where statistical methods are not applicable. But it is useless to deny that in the study of biological phenomena, as of any other natural phenomona where there are a large number of cases to be investigated, the statistical method is the only feasible one. Furthermore, the statistical method is quite as experimental and inductive as that of the Mendelians.

The preceding instance illustrates the tendency on the part of certain Mendelians, as, for example, Bateson and his followers, to exalt Mendelism to an unwarranted degree. To claim that Mendelism supplants Darwinism in any sense is entirely unjustifiable, while it certainly is preposterous to make any personal comparison between Mendel and Darwin which is in favor of Mendel, for, however much his theories may be disproved by the progress of biology, Darwin still remains the preëminent figure in biological science. As we have already seen, and as is admitted by the Mendelians themselves, Mendelism explains the origin and causes of variation no more than Darwinism. While it denies that selection is a cause of variation, it does not deny the potency of natural selection in determining the survival or extension of variations, as is indicated by Bateson himself in the following words: "There is also nothing in Mendelian discovery which runs counter to the cardinal doctrine that species have arisen 'by means of Natural Selection, or the preservation of favored races in the struggle for life,' to use the definition of that doctrine inscribed on 
the title of the Origin. By the arbitrament of Natural Selection all must succeed or fail." 1

It may further be pointed out that much that is discussed under the head of Mendelism is not peculiarly Mendelian. For example, we have seen that there have been numerous micromeric theories of the germ cell which postulate the existence of unit characters. A comparison has been made between Weismannism and Mendelism. It is evident that the determinants of Weismann are similar to the unit characters of Mendel. The purity of type which according to Mendelism results from segregation, according to Weismann is wrought out by a process of germinal selection. The dominant character of Mendelism may prove to be the stronger determinant of Weismann which succeeds in getting a larger share of nutriment in the intragerminal struggle.

\section{Pure Lines}

In this connection it may be well to speak of the theory of pure lines. A pure line consists of all the descendants of a single individual where the mode of reproduction has been by self-fertilization. Johannsen has studied the pure lines of barley, beans, and other plants and has found that in the inheritance of quantitative characters, as, for example, the weight of seeds, a pure line has a normal variability of its own. That is to say, the members of a pure line do not tend to revert to the mean of the general population from which the original self-fertilized ancestor of the pure line was taken, but tend to fluctuate around this individual ancestor as a mean. It has been found that the selection 1 Op. cit., p. 289. 


\section{The Anatomical and Physiological Basis 65}

of fluctuating variations within a pure line is not effective, but that the selected individuals tend to revert to the mean of their line. This would seem to furnish further evidence against the Darwinian theory of the selection of fluctuating variations as a cause of organic evolution. Furthermore, it raises the question of the mutability of unit characters. De Vries and others have laid great emphasis upon the immutability of unit characters. Morgan discusses this question as follows: -

"De Vries assumes that transitions between unit characters exist as little as between the molecules of chemistry. It cannot be maintained, I think, from the evidence that we possess, that unit characters are immutable, for there are some cases in which it appears that the unit characters may be halved by every crossing. It is true that some of these cases may be explained by antagonistic characters both developing and mutually influencing the result; but if they do not subsequently separate, it is impossible to tell whether or not a new unit character has been formed by combination. Cases of blended inheritance especially seem to come under this heading. But so long as we do not know definitely what occurs in these cases, it seems to me arbitrary to speak of unit characters as immutable and quite unnecessary to make this idea a cardinal point of the mutation theory. The behavior of certain characters in heredity shows that they do act as units, and it is a great convenience to deal with them as such, but unnecessary to push the matter so far as to hold that they are immutable. If unit characters can be halved, altered, added to, or changed in any way, their immutability does not seem to be an essential point of their characterization, and, as has been said, there is some evidence to indicate that such changes may take place."1

\section{Sex Determination}

The idea of unit characters has recently played an important part in the study of the determination of sex. For a long time the question of how sex is determined has been

1 T. H. Morgan, Experimental Zoölogy, New York, 1907, pp. 167-168. 
of great importance in biology. Many theories of sex determination have been formulated. One of the oldest theories has been that sex is determined by the nutriment of the parent or of the germ cells themselves. Other theories have been that it is determined by the age of the parents or by their vigor. Some theories have been based upon the condition of the germ cells. It has been claimed by some that in the case of certain mammals if the egg is fertilized soon after leaving the ovary, it tends to produce a female; if not fertilized until later, it tends to produce a male. Some have thought that in certain species the size of the egg is correlated in some way with sex, the large eggs producing females and the small ones males. It has been contended that sex is determined by the ratio of the nucleus to the cytoplasm. I cannot stop to discuss these theories, however interesting they may be, but wish to speak briefly of this idea of sex as a unit character which is now attracting a great deal of attention in biology. In most of the theories which have been mentioned the sex is not necessarily determined at the beginning of the life of the individual, namely, at the moment of conception, and the sex is not entirely determined within the germ cell. But according to the theory that sex is a unit character, it is determined at the moment of conception and entirely within the germ itself, no external forces playing a part in such determination.

This theory has grown out of the discovery of the socalled accessory or X chromosome, which seems to be the sex determinant in certain species. The chromosomes are minute particles of chromatin in the nucleus of the dividing germ cell which it has been thought are the bearers of unit 


\section{The Anatomical and Physiological Basis}

characters. There is a fixed number of these chromosomes for each species. But it has been found in certain species that half of the spermatozoa contain one chromosome less than the usual number for the species. Just before fertilization the number of chromosomes in both the egg and the sperm is reduced to half in the course of the process which is called the maturation of the germ cell. When fertilization takes place, the chromosomes of the egg and of the sperm are combined, and thus the fertilized germ cell contains the full number of chromosomes. If the sperm contains the accessory chromosome, the offspring will be female; if the accessory chromosome is absent, the offspring will be male. Thus the accessory chromosome seems to carry the character of femaleness, and the sperm cells may be classified as male or female producing according to the sex which they determine. It has also been thought that a similar classification could be made of the egg cells. This theory seems to explain the average equality between the sexes which has so far been very difficult to explain. Some of the Mendelians have taken up this theory and have claimed that sex is an allelomorphic unit character. The sexual individual would then be a Mendelian hybrid exhibiting in itself one sex, but carrying the potentiality of the other sex.

The theory that sex is a distinct unit character seems, however, to be contradicted by the fact that there are mixed sexual individuals known as gynandromorphs. Such cases appear most frequently among insects where an individual may display male characters on one side of the body and female characters on the other, or where the anterior end of the body is male and the posterior end female. Among 
plants a fully formed stamen sometimes appears upon a female flower. Functional mammary glands sometimes appear in the male mammal. These facts seem to indicate that there may be a mixing of sexual characters and that sex is not a distinct unit character, as is assumed by the above theory. At any rate, it is probable that sex is determined in different ways in different species. So that while sex may be determined morphologically in certain species, as is assumed by the unit character theory, it may be determined physiologically in other species by the general condition of the organism at the time of fertilization or shortly after.

\section{Biological Foundation for the Study of Behavior}

I have briefly surveyed some of the most important problems in biology to-day. The ultimate object of the study of each one of these problems is the explanation of the organic evolution now going on. The final explanation of this organic evolution must include a synthesis of the internal, orthogenetic forces of the organism and the external, environmental forces. The characters of an organism are determined initially by these internal forces. But whether or not a given individual or type is to survive and perpetuate itself depends upon these external forces which are always acting selectively upon all living organisms. Thus through the elimination of those not fitted to their environment, and a survival of those who are fitted, adaptation takes place. The raw material for organic evolution is furnished by the variations which take place within the organism. The greatest need to-day in biology probably is the study of the causes of these variations. 


\section{The Anatomical and Physiological Basis 69}

Some of these variations arise out of the internal conditions of the organism and do not result, at any rate directly, from the forces of the environment. Many other variations, however, are undoubtedly the direct result of external environmental forces, and there is great need for the experimental and statistical study of the effect of these forces upon the organism in causing variations.

This brief description of the evolution of structural forms and physiological processes furnishes the necessary biological foundation for the study of the evolution of behavior. It also furnishes the necessary biological basis for psychology and sociology, for, as we have seen, mental and social evolution consists in large part, if not entirely, of the evolution of behavior. It ought not to be necessary to prove that psychology and sociology must be based upon biology. In the first place, it is evident that there could have been no mental and social evolution had there been no organic evolution, for, so far as we know, mental and social phenomena characterize living organisms alone. Furthermore, biological forces are still at work and always will be at work in determining mental and social evolution, so that psychology and sociology can never be divorced from biology. In studying mental and social phenomena as evolving out of biological phenomena, and as being, broadly speaking, biological phenomena, we are using the genetic method. Not until the genetic method has been in large part substituted for the systematizing and classificatory method which has so far predominated in psychology and sociology can these sciences attain their full development or be generally recognized as natural sciences.

Biology has already played a considerable part in socio- 
logical writings. For example, the organic theory of society asserted that society is an organism like the biological organism. But it was soon proved not only that there is no identity between society and the biological organism, but that there is not even a close analogy between the two. The terms heredity, selection, adaptation, and variation have played an important part in sociological literature. But too frequently they have been regarded as distinct principles or forces at work in society as well as in the organism. For example, it has been asserted that heredity is the conservative force in society, while variation is the opposing radical force. The preceding discussion has, I believe, shown that while these terms may describe more or less aptly certain processes which are taking place, they cannot be regarded as distinct principles or forces in the organic any more than in the social world.

Darwinism is usually assumed nowadays as the biological basis in sociological writings. But we have seen that a new biology has grown up since Darwin and that many of his theories have been disproved, so that it is no longer safe to assume Darwinism without qualifications. Some of the errors into which sociologists frequently fall have been well described by an American biologist:-

"Much biological sociology rests on two very insecure bases: (I) a too slight acquaintance with biology on the part of the biological sociologist; and (2) an acceptance of, and confidence in, certain biological theories which are certainly unwarranted, and are not at all shared by the biologists themselves. Biological science contains much that is proved and certain; but also much that is nothing more than working hypothesis, provisional theory, and anticipatory generalization. As the proved part is largely of the nature of facts of observation, isolated and unrelated, and the unproved part is composed of the large and sweeping generalizations, the plausible, provisional ex- 


\section{The Anatomical and Physiological Basis}

planations, such as the various theories of heredity, of the results of struggle, of the development of mutual aid, etc., that is,. is exactly the sort of material that the sociologist needs to weave into his biological foundations for the sociologic study of man, it is exactly this unproved part of biology that the searching sociologist carries home with him from his excursions into the biological field. The recapitulation theory looms up large and familiar in biological sociology; it is mostly discredited in biology. The inheritance of acquired characters serves as basis for much sociology; most biologists believe it impossible. The selection theories are gospel to some sociologists; they are the principal moot points in present-day biology. And so on. Biology has not yet come to that stage in its development where it can offer many solidly founded generalizations on which other sciences can build. The theory of descent is one such safe great generalization; but perhaps Darwinism is not another. At least many scholars do not believe that it is." 1

The close of the above citation intimates that biology has not very much as yet to offer to other sciences. Sociologists should, however, make all possible use of present biological knowledge and await with great interest further biological discoveries. Bateson is more confident of the value of present biological knowledge for sociology, his confidence arising out of his high estimate of the importance of Mendelism :-

"It may be anticipated that a general recognition of the chief results of Mendelian analysis will bring about a profound change in man's conceptions of his own nature and in his outlook on the world. Many have in all ages held the belief that our powers and characteristics are directly dependent on physical composition; but when it becomes known that the dependence is so close that the hereditary descent of certain attributes can be proved to follow definite predicable formulæ, these ideas acquire a solidity they never possessed before, and it is likely that the science of sociology will pass into a new phase. The evidence at our disposal already proves that in many simple cases of defects and abnormalities the descent is of this

1 V. L. Kellogg, Darwinism To-day, New York, 1908, pp. 21-22. 
definite order, and it is scarcely doubtful that further research will reveal comparable examples in abundance. As regards more complex phenomena of human inheritance, the descent of characters involving the coincidence of several factors, and effects due to interference between factors, a complete analysis may be unattainable ; but even in some of these more obscure examples a close scrutiny will probably discover positive traces of regularity in descent of such a kind as to indicate in them also that the bodily or mental characteristic considered is a consequence of definite factorial composition. It is not in dispute that the appearance or nonappearance of a characteristic may be in part decided by environmental influences. Opportunity given may decide that a character manifests itself which without opportunity must have remained dormant. The question of opportunity and of the degree to which the conditions of life are operative in controlling or developing characters will some day demand attention, but in order to answer such questions successfully it is the first necessity that a knowledge of the genetic behaviour of the factors should be obtained." 1

The preceding discussion has shown that the whole theory of organic evolution must be taken into account by psychologists and sociologists, and not merely certain portions of it, as, for example, the theory of heredity. Mental and social phenomena have evolved in the course of organic evolution, and it is impossible to explain them fully except on the basis of the whole theory of organic evolution.

Biology is of peculiar interest with reference to man. It is probable that biological knowledge has not been used to the fullest extent in that branch of anthropology which deals with the anatomical and physiological characters of man, namely, physical or biological anthropology. Anthropology is now on a strictly Darwinian basis, but if the recent heterogenetic theories are true, man like other species may have originated as a mutation. The mutation theory 


\section{The Anatomical and Physiological Basis 73}

should therefore be thoroughly tested in anthropology. In similar fashion the different ethnic types may have originated as mutations, so that the mutation theory should be applied to them also in order to test its truth. Most of the so-called races of to-day are hybrids, so that the Mendelian law may assist us in studying the combinations of characters which these races exhibit. All these anthropological problems are very difficult, because our knowledge of the past is meager and because the human species changes very slowly. But the application of these new biological ideas to anthropological problems can be made with fruitful results, and there is a vast field for the experimental and statistical study of these anthropological problems in which this biological knowledge can be utilized.

Furthermore, this application of biological knowledge to anthropological problems will be of great value to psychology. The researches of Galton, Pearson, and others have shown that mental characters are not independent of inheritance. This was in any case a self-evident truth, for mental characters are caused by and correlated with biological characters, so it is evident that mental characters must be transmitted along with the biological. To trace the causal relationship is frequently a difficult thing to do, for a distinct mental character does not necessarily arise out of a single biological character, but may be caused by the combination of many biological characters comprising a large part or even the whole of the organism. The study of these psychological problems on a biological basis will undoubtedly help greatly in explaining the differences between the mental characters of different races and of the two sexes. 


\section{The Science of Human Behavior}

So far I have been discussing the significance of biology for psychology and sociology and the extent to which biological forces have determined mental and social evolution. But there has not been sufficient recognition in biology of the part that has been played by the mental and social forces in organic evolution. For while mental and social phenomena arose in the course of organic evolution, they have reacted upon organic evolution and have influenced it greatly. Some of the students of animal behavior have recognized this reaction, and I shall discuss it in later chapters. 


\section{CHAPTER V}

\section{THE BEHAVIOR OF THE LOWER ANTMALS}

Behavior as the dynamic, functional aspect of external organic phenomena, 75. - Application of the genetic method in psychology, 77. - The genetic method as the phylogenetic method, 77. - The development of the science of behavior by biologists and comparative psychologists, 77. - Experimental psychology, 78. - The irritability of organic matter arising out of its unstable and mobile nature, 79. Is this irritability a psychic characteristic? 79. - The direct reactions of organisms to external forces, 80 . - Definition of a tropism, $8 \mathrm{r}$. - The external forces which act upon organisms, 85 .

THE last two chapters were in the main morphological in their character. That is to say, they dealt with the forms and structures of organisms, but scarcely touched upon the incessant activities which characterize every organism. Organic phenomena were therefore treated in large part as if they were static phenomena. To be sure, variation and adaptation and the origin and evolution of the different species were discussed. But there was little indication that these changes resulted in part from the activities of the organisms, and the implication seemed to be that they were the result of external forces acting upon organisms in a passive state.

This has, I believe, been true altogether too frequently of general treatises on biology. There has been little in them to indicate that the activities of the organisms themselves have played a part in the organic evolution which 
they attempt to describe. This has, however, been far less true of treatises on physiology, for in them the activities and functions of the organs have necessarily been described so that the study has been much more dynamic in its character.

In the following chapters we are to deal with the dynamic, functional aspect of organic phenomena in the study of animal behavior. That such a study is not different in kind from physiology is indicated in the following citation from one of the leading students of animal behavior: "By behavior we mean the general bodily movements of organisms. These are not sharply distinguishable from the internal physiological processes. ... But behavior is a collective name for the most striking and evident of the activities performed by organisms." 1 Assuming, therefore, that behavior includes the external, more obvious physiological activities, it is evident that it must have an important morphogenetic rôle and that organic forms and structures must be determined in part by the behavior of the organisms they characterize. Modifications of behavior must therefore play a part in the modification of morphological characteristics. "The modifiability of the characteristics of organisms has always been a subject. of the greatest importance in biological science. In most fields the study of this matter is beset with great difficulties, for the modifications require long periods and their progress is not easily detectable. In the processes of behavior we have characteristics that are modifiable with absolute ease. In the ordinary course of behavior variations of action are continually occurring, as a result of many in-

${ }^{1}$ H. S. Jennings, Behavior of the Lower Organisms, New York, 1906, p. v. 
ternal and external causes. We see quickly and in the gross the changes produced by the environment, so that we have the best possible opportunity for the study of the principles according to which changes occur. Permanent modifications of the methods of action are easily produced in the behavior of many organisms. When we limit ourselves to the subjective aspect of these, thinking only of memory, or the like, we tend to obscure the general problem involved. This problem is: What lasting changes are producible in organisms by the environment or otherwise, and what are the principles governing such modifications? Perhaps in no other field do we have so favorable an opportunity for the study of this problem, fundamental for all biology, as in behavior. There seems to be no a priori reason for supposing the laws of modification to be different in this field from those found elsewhere. The matter needs to be dealt with from an objective standpoint, keeping the general problem in mind." 1 Behavior must therefore be studied in any complete survey of organic evolution.

The prime need in psychology to-day is the development of its genetic aspect. It is true that there has been a good deal written on what is called genetic psychology. But most of this has dealt with the psychic evolution of the human child alone. Genetic psychology should deal with the origin of psychic phenomena in general. In order to be thoroughly genetic, psychology must be phylogenetic. The evolution of psychic phenomena should be traced from the lowest species up to the highest. This work has been commenced by the comparative psychologists or students of animal behavior. Some of these are biologists and 
zoölogists, like Loeb or Jennings, who have been impressed with the part played in organic evolution by the activities of organisms. Others are psychologists, who have become interested in the mental processes of animals other than human beings. But the essential truth which most of these students of animal behavior have recognized is that psychic phenomena cannot be profitably studied except on the basis of a knowledge of the objective facts of behavior. We cannot pretend to know anything with much certainty about the psychic characteristics of any species, not even the human species, without being well acquainted with the modes of activity of that species. I propose therefore in the present and the two succeeding chapters to discuss the objective aspects of the behavior of the lower animals, relegating the discussion of the psychic or conscious aspects of such behavior to later chapters.

Many of the psychologists are giving their time now to experimental psychology. Abandoning the deductive and exclusively introspective methods of the older psychologists, they are using the inductive, experimental methods of modern science. But it is true of these psychologists, also, that they are devoting most of their time to the study of human beings. It is quite likely that genetic psychology will make much of this unnecessary by explaining certain phenomena which are now inexplicable and will, furthermore, show what experiments should be made.

If genetic psychology is developed as indicated above, the genetic method can be applied in psychology and sociology as it is now being applied in biology. These two sciences are now in much the same poșition as was biology during the Linnean period a century or so ago, namely, in the sys- 
tematizing period. The genetic method must be adopted before they can pass beyond this period and become fullfledged sciences.

In accordance with the genetic method the simplest reactions must be studied before the more complex. Consequently we shall discuss the reactions of the simplest organisms in this and the two succeeding chapters and take up the more complex reactions which characterize the nervous system, namely, the reflexes and the instincts, in later chapters.

\section{IRRITABILITY of ORGANIC MatTer}

It is, I think, generally believed that all the activities of living organisms arise primarily out of what is usually called the irritability of organic matter. I have already discussed this characteristic, without, however, mentioning its name, in the chapter on the physico-chemical basis of life. It arises out of the unstable and mobile nature of organic matter. It is at bottom nothing more than the facility with which organic matter changes as a result of the molecular and molar forces which act upon it. It is therefore a characteristic not different in kind, but only in quantity, for any matter will change as a result of the action of forces upon it. Many psychologists, however, and others also, have assumed that irritability is a psychic characteristic, and some have seemed to think that it marks an absolute distinction between organic and inorganic matter. Many illustrations of the belief that irritability is a psychic characteristic might be cited. For example, a French psychologist, Richet, speaks of the irritability of unicellular organisms as follows: "Irritability is their entire life, but it 
is already a psychic life; so that cellular irritability can be considered as the elementary psychic life." 1 Another French psychologist, Binet, reveals the same belief in a book in which he gives an account of the study of the behavior of unicellular organisms which had been made up to that time, but to all of which he gives a psychic interpretation. In the following passage he indicates his belief in the psychic characteristics of single cells and states that Haeckel had already revealed such a belief: "In giving to the psychology of these microscopic beings the name of cellular psychology, I have not invented a new expression, nor given a new sense to an old expression. M. Haeckel, a very long time before me, has made a study of cellular psychology, and his study rests entirely, like mine, upon the observation of the animal and vegetable organisms." 2

Haeckel seems to think that all matter possesses psychic characteristics. I do not know whether Binet is of the same opinion or thinks that psychic characteristics mark an absolute distinction between organic and inorganic matter. I think that both opinions are wrong and that psychic characteristics first appear in later stages of organic evolution. However, we are not concerned with the psychic in this chapter, but are to discuss irritability and the behavior of the lower organisms as purely objective phenomena.

What, then, are the simplest forms of behavior? They are the direct reactions of organisms to external forces. All forms of behavior are in the last analysis reactions to external forces. But in the higher organisms these reactions become more or less indirect. We must therefore

1 Charles Richet, Essai de psychologie générale, Paris, 1887, p. 20.

${ }^{2}$ A. Binet, La vie psychique des micro-organismes, $2 \mathrm{~d}$ edit., Paris, I89r, p. 23r. 
discuss first the cases where there is little to complicate the directness of the reaction to external forces.

\section{The Tropism}

Various names have been given to these direct reactions. Sometimes more than one name has been given to the same form of reaction. The best known of these names is that of tropism. A theory of tropisms has been developed which has stimulated a great deal of discussion and a good deal of difference of opinion. Because of this difference of opinion it is difficult to tell just what a tropism is. Let us see what are some of the definitions of a tropism.

A vcry simple definition of a tropism is the following: "The direct motor response of an animal to an external stimulus is known as a tropism, from the Greek word meaning "to turn.'" 1 It is evident that according to this definition the term tropism would cover most if not all of the direct reactions we have described. This is an example of a very broad conception of the meaning of tropism.

The most distinguished exponent of the tropism theory, or perhaps I should say, of a tropism theory, is the wellknown physiologist, Jacques Loeb. His conception of a tropism is more restricted and more complicated than the one illustrated above. He speaks of tropisms as follows: "These tropisms are identical for animals and plants. The explanation of them depends first upon the specific irritability of certain elements of the body surface, and, second, upon the relations of symmetry of the body. Symmetrical elements at the surface of the body have the

${ }^{1}$ Margaret F. Washburn, The Animal Mind, New York, 1908, p. 57. 
same irritability; unsymmetrical elements have a different irritability. Those nearer the oral pole possess an irritability greater than that of those near the aboral pole. These circumstances force an animal to orient itself toward a source of stimulation in such a way that symmetrical points on the surface of the body are stimulated equally. In this way the animals are led without will of their own either toward the source of the stimulus or away from it." 1 Thus we see that Loeb's tropism theory involves several things. It involves specialized forms of irritability on the body surface of the animal or plant concerned and symmetry of the organism. As a result of these conditions the organism is forced to orient itself toward the source of stimulation so that these symmetrical points will be stimulated equally.

Loeb's tropism theory has aroused a great deal of discussion and much disagreement. We find that even some of those who proclaim themselves disciples of Loeb do not define tropism as he does. For example, Bohn gives the following definition: "I give here to the word tropism the meaning that Loeb has attributed to it, and consequently I consider it as the simplest act that an animal can present." ${ }_{2}$ It is evident that this definition does not necessarily involve specific irritability, symmetry, or orientation, as does Loeb's definition.

Davenport seems to regard as a tropism any modification of the direction of growth of an organism by an external force, for in the second volume of his work on experi-

1 Comparative Physiology of the Brain and Comparative Psychology, New York, 1900, p. 7. Cf. Loeb, The Dynamics of Living Matter, New York, 1906, pp. 138-139.

2 Georges Bohn, Les tropismes, les reflexes, et l'intelligence, in L'année psychologique, edited by A. Binet, Paris, 1906, p. 147. 


\section{The Behavior of the Lower Animals}

mental morphology he deals with the "effect of chemical and physical agents upon growth" and each of these modifications he calls a tropism. In the first volume of this work he deals with the "effect of chemical and physical agents upon protoplasm" and discusses most of the time the determination of the direction of locomotion of an organism by external forces, which he calls "taxis." It is evident that this is similar to Loeb's use of the term tropism and we shall discuss its meaning shortly.

Jennings describes the diversity of meanings which have been attributed to the word tropism, but believes that one meaning is generally accepted. "The word 'tropism' has been used in several different senses by different authors, and not always as implying a definite theory. But there is a certain theory which is usually implied when tropisms are mentioned; it has become so generally accepted that it is often spoken of as the tropism theory. It will perhaps be more accurate to speak of it as the local action theory of tropisms." 1 By this he means a tropism theory which assumes that external forces determine behavior by affecting in a different fashion the different parts of the organism, and sometimes affecting some parts of the organism not at all. Loeb's theory is of this kind, as is indicated in the definition which has been quoted in which he says that the explanation of tropisms depends upon "the specific irritability of certain elements of the body surface." After criticizing this local action theory of tropisms (a criticism which will be discussed later), Jennings says: "In the foregoing pages we have criticized a certain definite theory of tropisms, this being the theory most commonly implied 
when the word is used in a precisely defined way. But the term 'tropism' is often used in a looser sense. By some writers the word is applied merely to the general phenomenon that the movements of organisms show definite relations to the location of external agents." 1

Another term which is applied to certain reactions is the word taxis, which is derived from the Greek word meaning "to arrange." By some writers taxis is used in the same sense as tropism. ${ }^{2}$ For example, Verworn speaks as follows : "Although the words 'chemotropism,' 'heliotropism,' etc., have been long in use, I have decided, after considerable delay, to exchange them in this edition of the book for the words 'chemotaxis,' 'phototaxis,' etc.; my reason is that the former not only sound heavy, but suggest objections from the philological standpoint." 3 He does not state what are the philological objections to the term tropism, but he evidently thinks that tropism and taxis mean the same thing.

Other writers use the two terms with different meanings. "By some writers the word 'tropism' is restricted to the bending or inclination of a fixed organism, while the movements of free organisms under the influence of external agents are called taxis." 4 Davenport would, I think, be an example of this class, for, as we have seen, he applies tropism to modifications of the direction of growth by external forces, which is true principally of fixed organisms such as plants, while he applies taxis to the determination of the direction of locomotion by external forces, which would be true only of free organisms.

1 Op. cit., p. 274. $\quad 2$ Cf. Washburn, op. cit., p. 57 ; Jennings, op. cit., p. 275.

- Max Verworn, General Physiology, London, 1899, p. 429.

4 Jennings, op. cit., p. 275. 


\section{The Behavior of the Lower Animals}

Many other terms have been applied to the reactions of the lower organisms, such as kinesis, -pathy, -metry, phobism, clinism, etc. We shall not stop to define and discuss these terms for the following reason: On account of the multiplicity of these terms and the diversity of meanings which have been given to them, there has resulted a good deal of confusion of thought and the obscuring of the real nature of the phenomena involved. We shall therefore use as few of these terms as possible and shall devote ourselves rather to the study of the reactions themselves. The attempt to devise a technical nomenclature for these reactions is a commendable one, for it arises out of the desire to describe these phenomena in as precise and objective terms as possible. But, as Jennings says, "the study of behavior seems hardly to have reached as yet the stage where a hard and fast nomenclature can be used to advantage. To the present writer, after a long-continued attempt to use some of the systems of nomenclature devised, descriptions of the facts of behavior in the simplest language possible seems a great gain for clear thinking and unambiguous expression." 1

\section{External Forces as Stimuli}

We shall turn now to the study of the reactions of the simplest organisms to external forces. What are these forces? Davenport classifies them as follows: "These may be grouped into eight categories, determined largely by convenience ; namely, I, chemical substances ; 2, water ; 3 , density of the medium; 4, molar agents; 5 , gravity; 6 , electricity ; 7 , light ; and 8 , heat." 2

$$
\text { 3Op. cit., p. } 276 . \quad 2 \text { Op. cit., p. viii. }
$$


Verworn gives a somewhat briefer classification of these external forces which he calls stimuli. First he defines a stimulus as follows: "A stimulus may be defined as every change of the external agencies that act upon an organism. If a stimulus comes in contact with a body that possesses the property of irritability, i.e. the capability of reacting to stimuli, the result is stimulation." 1 Then he discusses the varieties of stimuli as follows: "If every change of the agencies that act upon the organism from without is able to stimulate, it is evident that innumerable kinds of stimuli exist. Not only may every existing condition of life be changed, but new conditions may appear and affect the organism. Notwithstanding this possibility, stimuli may be classified according to their qualities into a few large groups. A natural classification is possible in accordance with the forms of energy which the different stimuli represent; for the operation of every external agent upon a body depends upon a transformation of energy. In accordance with this principle, all influences of a chemical nature may be grouped as chemical stimuli, including not only changes in the income of food, water, and oxygen, but other chemical changes which ordinarily do not come into contact with the organism. . . . All purely mechanical influences that affect the organism may be termed mechanical stimuli, including those that consist in changes of pressure, such as pushing, shaking, pressing, pulling, and sound vibrations, those that manifest themselves by molecular attractions, such as cohesion or adhesion in the surrounding medium, and those that depend on the action of gravitation. Thermal stimuli comprise

$$
{ }^{1} \text { Op. cit., p. } 348 .
$$


changes of the temperature that surrounds the organism. Photic stimuli comprise changes of light. Electrical stimuli comprise electrical changes. . . . Stimuli, therefore, comprise chemical, mechanical, thermal, photic, and electrical changes in the environment of the organism, and no others." 1

These two classifications are sufficient to suggest the forces which act upon organisms and we shall now turn to the study of the behavior which results from such action.

1Op. cit., pp. 348-349. 


\section{CHAPTER VI}

\section{TROPISMS}

Phototropism, 88. - Photopathy, 92. - Does the direction or the intensity of the rays of light determine the reactions of organisms to light? 93. - Positive and negative phototropism, 95. - Is the action of light on organic matter mechanical or chemical? 99. - Chromotropism, I02. - Rudimentary vision, 105. - The utility of reactions to light, 105. - Geotropism, 107. - The force of gravity, 108. Chemotropism, I09. - Rudimentary senses of smell and taste, III. Galvanotropism, I I2. - Barotaxis, II3. - Stereotropism (thigmotropism) or contact irritability, II3. - Rheotropism, II4. - Anemotropism, I 6 6. - Thermotropism, I I6. - Hydrotropism, I 6 6. - Tonotropism, II6.

THE first group of reactions to external forces I shall discuss is that of reactions of organisms to light. ${ }^{1}$ Organisms are being subjected to light pretty constantly, and their reactions have been studied a great deal, so that the reactions of organisms to external forces are well illustrated in this group.

\section{PhototropisM}

It has long been a well-known fact that certain plants turn their flowers, leaves, etc., towards the sun or any other source of light, while other plants turn away from light. Such reacting to light has long been called heliotropism, a word derived from the Greek words for "sun" and "to turn." When the turning is towards the source

1 Since the present chapter was written there has appeared an excellent summary of this subject in S. O. Mast, Light and the Behavior of Organisms, New York, 1911. 
of light, it is called positive heliotropism; when it is away from the light, it is called negative heliotropism. It has also long been known that certain of the lower organisms react in similar fashion towards light, as, for example, the moth, which flies towards a source of light, such as a flame, and the earthworm, which crawls away from the sunlight. Various anthropomorphic interpretations of such phenomena have been offered, as, for example, that a plant turns towards the light because it loves the light and that a moth flies into a flame out of curiosity. For a long time, however, the reactions of plants and of animals to light were not identified as being the same. It goes without saying that these anthropomorphic explanations are not tenable, and I shall now discuss more scientific explanations of these reactions.

But first a word must be said with regard to nomenclature. Strictly speaking, the word "heliotropism" refers only to reactions to sunlight. This fact has encouraged the introduction of special terms, such as "selenotropism," or turning towards the moon. To be sure, heliotropism has been used in the broad sense of a reaction to all kinds of light, as, for example, by Loeb. But the term "phototropism" has been devised, which expresses this broad sense very well and which I shall therefore use. The term "phototaxis" is also used by some in this same broad sense, though usually the meaning of this term is somewhat restricted in other respects.

The reactions of plants to light have been studied for a long time, and it has long been recognized that the curvature of plants in response to light is the result of unequal growth. "It is clear that phototropic curvature, as seen 
in the seedling, the mold, or the hydroid, is the result of unequal growth upon the two sides of the cylindrical organ; and, indeed, that the positive phototropism is due to a relative diminution of growth on the side next the source of light, and the negative phototropism to a relative increase of growth on that side. Experiments have shown that in positive phototropism growth is excessively rapid upon the convex side of the organ, and excessively slow upon the concave side. These results are reasonably attributed to an increased turgescence on the one side and a decreased turgescence on the other." 1

Some of the earlier investigators of heliotropism in plants, such as von Sachs ${ }^{2}$ and Strasburger, ${ }^{3}$ believed that the orientation of plants towards the sun was determined by the direction of the rays of light and their refrangibility. Loeb conducted some experiments which, he believed, proved that the reactions of the lower animals to light are determined in the same way. "In a former paper I showed that the dependence of animal movement upon light is identical with that of plants on the same source of stimulation. I showed that the law put forward by Sachs for the heliotropism of plants, namely, that the direction of the rays of light determines the orientation, holds good also for animals. Free-moving animals are compelled to execute their progressive movements in the direction of the rays of light, as is the case with the swarm spores of certain Algæ. It was further proved that the more refrangible

'C. B. Davenport, Experimental Morphology, New York, 1899, Vol. II, pp. 444-445.

2 J. von Sachs, Vorlesungen über Pfanzen-Physiologie, 2 d edit., Leipsic, 1887.

${ }^{3}$ E. Strasburger, Wirkung des Lichtes und der Wärme auf Schwärmsporem, Jena, 1878 . 


\section{Tropisms}

rays of the visible spectrum are the rays that are solely, or at least chiefly, effective in bringing about the movements of these animals; as is the case in the heliotropic movements of plants." 1

But many investigators of the subject have denied that the direction of the rays of light determines the movements of organisms, but that on the contrary it is the intensity of the light. For example, Verworn has opposed Loeb's theory, as is indicated in the following passage: "From the preceding consideration and by analogy with the directive effects of other stimuli it is evident that only the difference in the intensity of the light upon different parts of the body can produce a directive effect; where the stimulus acts upon the surface of the body from all sides with equal intensity, the reason for a definite axial position disappears, as is to be observed most clearly in the action of chemical stimuli upon all sides. Although this is obvious, some investigators, such as Sachs and Loeb, have believed that the direction of the rays is more responsible for the manifestation of phototactic phenomena than are differences in intensity. It is difficult to conceive this, for, since the assumption of an axial direction is possible only when differences exist at two different points of the surface of the body, it is wholly mystical how the directions of the rays, which is the same on all sides of the body, can produce such an effect." ${ }_{2}$

It is true that Loeb also had recognized the reactions of organisms to differences in the intensity of light, as he

1 Pfiügers Archiv, Vol. XLVII (1890), p. 39r, translated in Studies in General Physiology, Chicago, 1905, Vol. I, p. 89.

${ }^{2}$ Max Verworn, General Physiology, London, r899, pp. 450-45I. 
insists in the following passage: "I showed first, that the orientation and the direction of the progressive motion of certain animals can be controlled unequivocally by the direction of the rays emanating from a source of light, and I showed, moreover, that this type of reaction is, as far as we can judge, in every point identical with the heliotropic reaction of plants. A few years later I showed that there exists another group of animal reactions to light, which is not covered by the theory of tropisms, but which depends upon the rapidity of the change of the intensity of the light. This latter type of reaction I designated as Unterschiedsempfindlichkeit." 1 However, this passage seems to indicate that Loeb still believes that some at least of the reactions of organisms are determined by the direction of the rays of light.

Reactions determined by the direction of the rays of light have sometimes been called phototaxis, and those determined by the intensity of light photopathy. Davenport defines these terms as follows: "In this section we shall deal with two sets of phenomena which very likely are different, but which, in our ignorance, we cannot always distinguish. The first includes that active migration of organisms whose direction is determined by that of the rays of light. This is phototaxis. The second includes the wandering of organisms into a more or less intensely illuminated region, the direction of locomotion being determined by a difference in intensity of illumination of the two poles of the organism. This is photopathy." 2

${ }^{1}$ Concerning the Theory of Tropisms, in the Jour. of Experimental Zoölogy, Vol. IV, p. I5I (Feb. 1907).

2 C. B. Davenport, Experimental Morphology, New York, I897, Vol. I, p. I80. 
There has been a long controversy over this question as to whether the reactions of organisms to light are determined always by the intensity or sometimes by the direction of the rays of light. It is possible that the direction of the rays has an effect in orienting the organism, but the weight of opinion now seems to be that it is the intensity which always determines it. The direction does, to be sure, have something to do with it, because the intensity of the light at different points on the body is determined by the direction from which the light is coming. But it is the intensity which determines the activity of the different parts of the body. That is to say, if an organism is symmetrical so that corresponding points on the two sides of the body are equally sensitive to the light, and if the light is coming from one side, the other side will be in the shade and will therefore not be as intensely illuminated as the first side. Under such conditions the muscles or other locomotor organs on the light side will probably be stimulated to greater activity, so that the organism will be pulled around into a position parallel with the rays of light. In such a case it might easily appear as if the orientation was being effected by the direction of the rays of light, whereas in reality it was caused by the unequal intensity on the opposite sides of the body. If this theory is true, there is little if any difference between phototaxis and photopathy. In both cases the intensity of the light is the final cause of the reaction. In the first case the organism is definitely oriented with respect to the source of the light, while in the second case it would not be definitely oriented. Phototaxis would then exist when the light was coming from one direction, while photopathy 
would exist when the light was evenly diffused. In the second case the organism would be restless, and the orientation would be effected by wandering about until it came into a region of different intensity. If the intensity in this region was greater, the organism would remain there if it was positively photopathic. If it was negatively photopathic, it would not come to rest until it reached a region of less intensity.

Holt and Lee ${ }^{1}$ made some experiments to test this theory and came to the conclusion that there is no difference between phototaxis and photopathy. I have not sufficient space to give a full account of their investigation, but can only quote their conclusions. "The direction of the rays has, in itself, no effect whatsoever on movements of organisms. It is true, however, that if the rays reach the animal from a certain side, that side of the body is stimulated more than the other, for the other side lies in its own shade." 2 "Light acts in one way, that is, by its intensity. The light operates, naturally, on the part of the animal which it reaches. The intensity of the light determines the sense of the response, whether contractile or expansive; and the place of the response, the part of the body stimulated, determines the ultimate orientation of the animal." 3 "A given portion of an organism stimulated by a given intensity of light will respond, so far as is shown by the facts hitherto observed, by orienting the organism in a particular way. The facts do not show that the direction of the ray is otherwise effective than in determining on what part of the animal the light shall fall. There is no evidence that

1 E. B. Holt, and F. S. Lee, The Theory of Pholotactic Response, in the Am. Jour. of Physiology, Vol. IV, pp. $460-48$ I (Jan. 1901). $\quad$ 2 P. 480 . Pp. $480-481$. 
organisms respond to any other property of light than its intensity, and the distinction commonly made between phototaxis and photopathy as different forms of irritability is unwarranted." 1

Jennings ${ }^{2}$ also has made some experiments which throw light on this subject. He chose for his experiments two species of the protozoön group, infusoria. One of them was the Stentor cceruleus, which reacts negatively to light. This is an unsymmetrical organism which propels itself by means of cilia. He found that the stentors swim about in all directions in the dark. But as soon as one of them comes to the lighted area it at once reacts by what he calls the "avoiding reaction." That is to say, it swims backward and turns toward the right aboral side. Then it moves forward on a new course. If this again brings it into the light it gives again the avoiding reaction and continues to do so until it no longer comes into the light. This is what Jennings calls the "method of trial" which he believes characterizes much of the behavior of the lower organisms. This will be discussed in the next chapter. He believes that the stentor responds with this avoiding reaction to light because its anterior, oral end is very sensitive to light so that it is forced to withdraw its head from the light. It does not turn and swim parallel with the rays of light, so that its orientation is not determined by the direction of rays of light. "The orientation of the free Stentor in line with the light rays, with its anterior end directed away from the source of light, is due to the fact that an increase of illumination at the sensitive anterior end induces the avoiding reaction. As a necessary result the oriented

1 P. 48r. $\quad 2$ Behavior of the Lower Organisms, New York, I906, pp. 128-r41. 
Stentor, swimming in a spiral path, tries new directions of movement until it finds one where such changes of illumination no longer occur. Such a direction is found only in orientation with the anterior end directed away from the source of light." 1

Loeb, who, as we have seen, is the leading exponent of the tropism theory, has pointed out ${ }^{2}$ that he had recognized long ago this type of reaction to light and had described it in the case of planarians and earthworms. "Such animals become more quiet when the intensity of light is rapidly diminished, become more active when the intensity of light is suddenly increased. The consequence is that places of a relative minimum in the intensity of light act like a trap upon them." ${ }_{3}$ He insists, however, that the existence of these cases of Unterschiedsempfindlichkeit, as he calls them, or of photopathy, as they have been called by others, ${ }^{4}$ does not disprove the existence of the cases where the reactions are determined by the direction of the rays of light.

The other species of infusoria which Jennings chose for experiment was the Euglena viridis, which reacts positively to light. This is a relatively unsymmetrical organism which propels itself by means of a flagellum fastened at its anterior end. Near the anterior end it has a red pigment spot known as the eye spot. Because of its unsymmetrical shape it swims in a spiral course. Euglenæ gather in

1 Op. cit., p. I34.

${ }^{2}$ Concerning the Theory of Tropisms, in the Jour. of Experimental Zoölogy, Vol. IV, p. 155 (Feb. 1907).

Op. cil., p. 155 .

4 This response to changes in the intensity of light has also been called "reaction to change" by Jennings, in the Jour. of Comp. Neurology and Psychology, Vol. XIV, pp. 464-468 (1904), and "sensibilité différentielle" by Georges Bohn, La nouvelle psychologie animale, Paris, 1911. 


\section{Tropisms}

lighted areas where the light is not too strong. If one of them swims into a dark region, it immediately gives the avoiding reaction and moves forward in another direction. If this again brings it into the dark, it gives the avoiding reaction again and continues to do so until it moves forward into the light. If light is thrown upon it from one side, it does not turn immediately towards it. But as it swings to the right and to the left in its spiral course it will tend to swing more towards the source of the light than it will in the other direction. "The result is that in its spiral course it successively swerves strongly toward the source of light, then slightly away from it, until by a continuation of this process the anterior end is directed toward the light. In this position it swims forward." 1 Jennings contends that this reaction is entirely the result of the effect of the intensity of the light on the sensitive oral end of the organism. But it is true that this reaction has the appearance of being determined by the direction of the rays of light, and consequently Jennings has been criticized

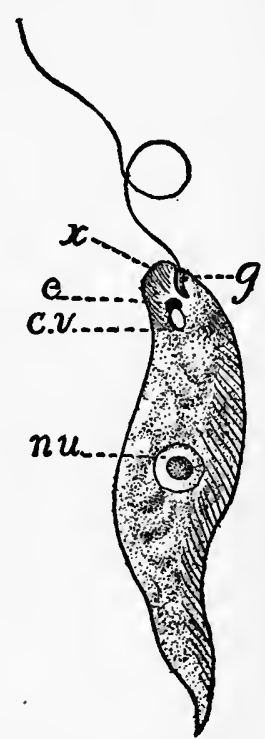

FIG. r.-Euglena viridis. c.v., reservoir of the contractile vacuole; $e$, eye spot; $g$, gullet; $n u$, nucleus; $x$, larger or upper lip. (From Jennings, after Kent.) for this explanation of it. For example, Torrey has claimed that this is a case of a reaction being determined by the direction of the rays. In an extended criticism of Jennings he speaks as follows: "It is hard for me to conceive how an organism swimming of necessity in a spiral course could 1Op. cit., pp. 138-139. 
react more definitely to a moderate directive stimulus than does Euglena here." 1 Jennings has replied to this criticism by saying ${ }^{2}$ that in this experiment when the organism is subjected to light from one side, the swinging in the spiral course increases towards both sides, but it increases more towards the light, and this is why it becomes oriented in course of time towards the source of light. He contends that the increased swerving towards the light can be explained as a tropic reaction, but that the increased swerving away from the light cannot possibly be explained by the tropism theory. So he concludes that the orienting is accomplished entirely by the intensity and not at all by the direction of the light.

Jennings' conclusion from these two experiments reads as follows: "Thus in both negative organisms (Stentor) and positive organisms (Euglena), the determining cause of the reaction is a change in the intensity of light, and the reaction takes place by the usual method of the performance of varied movements, subjecting the animal successively to different conditions. When the sensitive anterior end is subjected alternately to light and shade, the organism 'tries' other directions of movement till it finds one where such changes are not produced. In Stentor it is an increase of light that causes this reaction; in Euglena it is usually a decrease that causes the reaction, though when the light is very strong an increase may have the same effect." 3

\footnotetext{
${ }^{1}$ H. B. Torrey, The Method of Trial and the Tropism Hypothesis, in Science, Vol. XXVI, p. 317 (Sept. 6, 1907).

${ }^{2}$ The Interpretation of the Behavior of the Lower Organisms, in Science, Vol. XXVII, p. 705 (May I, I908).

${ }^{3}$ Op. cit., p. I4I. Cf. S. J. Holmes, The Selection of Random Movements as a Factor in Phototaxis, in the Jour. of Comp. Neurology and Psychology, Vol. XV, pp. 98-II 2 (I905).
} 


\section{Tropisms}

I have no space to discuss this subject further here, and it would be worse than foolish to express an opinion as to this controverted point which can be settled only by means of further experimenting. I will only repeat the statement that the weight of opinion now seems to be that the direction of the light does not in itself affect the activity of the organism, but that it may influence the orienting of the organism when it determines the intensity of the illumination of the different parts of the body so that if the organism is symmetrical, or if the anterior, oral end of the body is more sensitive than the posterior end, the organism becomes oriented towards or away from the source of light. I shall touch briefly again upon this subject when the tropism theory in general is discussed, but we shall now have to turn our attention to certain other aspects of the reactions of the lower organisms to light.

The first question we must take up, and it is indeed the most important one in the whole subject, is as to how light is able to affect so greatly the movements of organisms. The light rays, which, according to the most recent theory in physics, are wave movements in the ether, are impinging upon the surface of the organism and are apparently causing certain changes therein. These changes may be of two sorts. They may be chemical changes in the bodysurface of the organism which in turn affect the movements of the organism. Or they may be purely mechanical changes, in the sense that they push or pull muscles, locomotor organs, or other parts of the body in such a fashion as to affect the movements of the organism. The most popular theory seems to be that these changes are chemical. This sounds plausible, to say the least, in the case of the 
reactions of plants to light. It has already been noted that the heliotropic reactions of plants are in all probability caused by unequal growth in different parts of the plant. If this is the case, the light is affecting the metabolic process, and this process is a chemical one. Davenport is very certain that the effect of light on organisms is a chemical one. He speaks first of its effect on protoplasm in general. "The reactions produced by light upon protoplasm are undoubtedly of a chemical character, and, indeed, experiments with nonliving organic compounds show that it has an important effect in synthesis, in analysis, in substitution, in the production of isomeric or polymeric conditions, and in fermentation." 1 Later he speaks of its effect upon the movements of organisms. "We thus see that organisms respond to light, and that this response, exhibited in movements, is not of a widely different order from the disturbances produced in metabolism, which in turn are of the same order as the chemical changes produced by light in our laboratories upon nonliving substances. In a word, response to light is the result of chemical changes in the protoplasm wrought by light." 2

Loeb also insists over and over again that light affects the movements of organisms by causing chemical changes in the body surface of the organism. For example, in trying to account for the heliotropic curvatures of plants, he speaks as follows: "Let us suppose that light strikes a plant on one side only, or more strongly on one side than on the opposite side, and that it be absorbed in the superficial layers of tissue of that side. In this case we assume that on that side certain chemical reactions occur with

1 Experimental Morphology, New York, 1897, Vol. I, p. $210 . \quad 2 O p$. cit., p. 211. 


\section{Tropisms}

greater velocity than on the opposite side. What these reactions are is unknown; we may think provisionally of oxidations. This change in the velocity of chemical reactions either produces a tendency of the soft elements on that side to contract a little more than on the opposite side, or creates otherwise a greater resistance to those forces which have a tendency to elongate or stretch the plant, e.g., hydrostatic pressure inside the cells, or imbibition of certain tissue elements. The outcome will be that one side of the stem will be stretched more than the opposite side, and this will bring about a curvature of the stem." 1 So far he has been speaking of the positively heliotropic parts of plants. Later he speaks as follows of the negatively heliotropic parts: "The same reasoning applies also to negatively heliotropic organs, e.g., roots, with the difference only, that in the latter case the photochemical effects result in a relaxation or a decreased resistance to the stretching forces on that side of the organ where the light strikes." ${ }^{2}$ According to Loeb, then, light causes certain chemical changes which lead to certain mechanical results in the way of contractions and relaxations which influence the movements of organisms. It should be noted that he states that the exact nature of these chemical reactions is not yet known, and it is well to reserve judgment as to the causes of these phototropic movements in plants until more is known about them.

When we come to the study of the reactions of animals to light, it seems less probable that the changes caused by light are always chemical. Loeb seems to be certain that they are always chemical, as indicated in the following

${ }^{1}$ The Dynamics of Living Matter, New York, I906, p. II8. $\quad{ }^{2}$ Op. cil., p. IIg. 
passage: "First, heliotropic animals as well as heliotropic plants must contain a substance on their surfaces which undergoes a chemical change when subjected to the influence of the light, and this change must be able to produce changes of tension in the contractile tissue. Second, heliotropic animals as well as heliotropic plants possess symmetry of form and a corresponding distribution of the irritabilities. These two groups of conditions determine the heliotropic reaction unequivocally." 1 But it may be that in some cases the reaction is entirely mechanical. For example, in the case of these microörganisms which propel themselves by means of cilia or a flagellum, it may be that the light waves, without causing any chemical change in these locomotor organs, stimulate them to greater activity by pushing them. The light would in such cases act like a molar force.

\section{Chromotropism}

The reactions of organisms to color, which are usually called chromotropism, are of importance in this connection. The color sensations are caused by the differences in the length of the light waves. It is to be expected, therefore, that the different colors would act differently upon organisms, chemically and perhaps mechanically also. In the following passage, Davenport indicates some of the chemical effects of the different colors: "Upon metabolism we can distinguish an effect of the red rays, which are greatly absorbed by chlorophyll and are chiefly active in assimilation, and an effect of the blue rays, which seem to produce important chemical changes, increasing the production of carbon dioxide in plants, creating an electric current in the

1 Comparative Physiology of the Brain and Comparative Psychology, New York, I900, p. 183 . 
retina as it falls thereon, and bleaching visual purple." 1 Loeb describes some of these chemical effects more fully: "We do not yet know with the same degree of certainty, as in the case of the process of assimilation, the relative heliotropic efficiency of each part of the spectrum; but from experiments with colored screens it appears that the more refractive green, blue, and violet rays of the spectrum are more effective heliotropically than the less refractive red and yellow rays. There exists thus apparently a division of labor, the longer light waves accelerating assimilation, the shorter waves accelerating heliotropism. This can be demonstrated with the aid of screens, inasmuch as behind red screens the plants assimilate well, while they do not bend or bend only slowly, toward the source of light; while behind a blue screen they bend actively toward the light, their assimilation being diminished." 2 Later on he shows in the case of certain animals that the same holds true for animals. "It can also easily be shown that in these animals, just as in plants, the more refrangible blue rays are more effective than the red rays, and that the latter act like weak light." ${ }^{3}$ It appears, then, that the refrangibility of the rays of light has an effect upon the chemical and perhaps also upon the mechanical reactions to light. Further investigation of chromotropism will therefore undoubtedly throw light upon the general subject of the reactions of organisms to light. ${ }^{4}$

I Experimental Morphology, New York, r897, Vol. I, p. 2 r I.

${ }^{2}$ The Dynamics of Living Matter, New York, r9o6, pp. I $17-118$.

O Op. cit., p. 127.

- Professor G. H. Parker has been engaged for several years in working out a method for producing colored light of good quality and measured intensity, with which to test the reactions of organisms to color. He has not yet, at the time of this writing, published the results of his investigation. 
For each species there is a certain optimal intensity of light which is most favorable to it. Jennings has defined the "optimum" and suggested how organisms reach it in the following passage: "The organism generally reacts by a change in its behavior when the change is of such a nature as to lead away from the optimum. By optimum we mean here the conditions most favorable to the life processes of the organism in question. Changes leading toward this optimum produce in many animals no reaction; the organisms simply continue the activity which has brought about this change. Changes leading away from the optimum produce a negative reaction, by which the organism is removed from the operation of this change." 1 An intensity of light much above or much below the optimum is injurious to the organism. Each species has its own means for getting away from the intensity which is injurious to it and for reaching the optimum. I do not, however, mean to imply by this that the organism consciously seeks its optimum, for these organisms are too simple to be conscious of a need for the optimum. But those organisms which do not react negatively to the light conditions which are injurious to it would tend to be eliminated, so that the species which survive tend to be those which react adaptively to light. Thus each species has its own means for reaching the optimal intensity, plants by bending the stalk, animals by contraction or relaxation of muscles, by movements of cilia or flagella, etc. Not all species, however, react so readily to light. For example, most colorless infusoria do not react to light of ordinary intensity. ${ }^{2}$ It is, however, hardly conceivable that there is any species which 
would not react under any light conditions. There is probably no species which would not react to extreme light, while the same is likely to be true for extreme absence of light. Extreme light would certainly have a destructive effect upon the tissues of the body, while extreme darkness would probably interfere with the metabolic processes.

Nothing has been said about vision in this discussion of the reactions of these microörganisms to light. Some of these organisms have pigment spots which may be rudimentary eyes. Experiments have been made to determine whether these spots play a part in determining the reactions of these organisms to light. It appears that they do in some organisms, but apparently not in the case of others. This subject is of most importance in connection with the origin and evolution of the visual sense, so that I shall discuss it briefly in the chapters on the nervous system.

\section{Utility of Reactions to Light}

The last topic we have to discuss under the head of the reactions of these microörganisms to light is the functional value of these reactions. It has already been indicated that these reactions are subjected to a process of natural selection so that only those species tend to survive whose reactions to light favor the life processes. I will now give a few illustrations of the functional value of these adaptive reactions.

One of the annelids, the earthworm, is negatively phototropic, so that it burrows in the ground during the day and comes out only at night to feed and to have sexual intercourse. This form of reaction to light seems to be advantageous, for if it came out during the day it would be 


\section{The Science of Human Behavior}

likely to be eaten by birds. ${ }^{1}$ In similar fashion the freshwater planarian is negatively phototropic, so that it stays under stones during the day and comes out only at night to feed. This form of reaction to light seems to be advantageous, for if it came out during the day it might be eaten by its enemies.

The larvæ of Porthesia chrysorrhoea, ${ }^{2}$ which are the young caterpillars of a certain kind of butterfly, come out of their eggs in the nest on a tree or shrub and climb up to the tops of the branches and twigs because they are positively phototropic. Thus they are able to feed on the tender tips of the twigs and upon the buds and leaves. After they have eaten sufficiently they become negatively phototropic and crawl down again. The character of the phototropism is changed by the changes in the chemical composition of the skin of the animal caused by the metabolic process. This is one of the numerous cases in which changes in the organism caused by the internal processes or induced by external forces cause changes in the form of the reaction of the organism to light.

In the case of certain winged ants both the male and female ants become positively phototropic at sexual maturity, which causes them to take the "nuptial flight," during which they pair in the air. ${ }^{3}$

Reactions to light are not always, however, advantageous. They may be indifferent, or may sometimes be positively injurious. For example, certain night-flying insects are

1 This and several of the following cases are described in T. H. Morgan, Evolution and Adaptation, New York, 1903, chap. XI.

2 J. Loeb, Comparative Physiology of the Brain and Comparative Psychology, New York, 1900, pp. 188-190.

'J. Loeb, Studies in General Physiology, Chicago, 1905, p. 52. 


\section{Tropisms}

phototropic. Whether or not this phototropism is ever advantageous to them, is hard to determine. But sometimes it is very injurious, as when the moth flies into the flame of a lamp or any other source of light because it is moving too rapidly to turn aside. However, as has been suggested above, no species can long survive with reactions to light which are fatally disadvantageous to it, so that most species are characterized by reactions which have an adaptive value.

The form of the reaction of a species to light may vary greatly, and such variations sometimes have great adaptive value. I have already suggested how the metabolic process may cause such variations. Various other causes might be mentioned, such as the period of life of the organism, continued exposure to light, which may modify the response to light, the temperature, the concentration of the medium, the chemical condition of the medium, etc. ${ }^{1}$

\section{GEOTROPISM}

One of the forces to which organisms are being constantly subjected is that of gravity. The reaction to gravity is usually called geotropism, sometimes geotaxis. If the reaction is towards the earth, it is positive; if it is away from the earth, it is negative. Gravity seems to have most influence upon the growth of sessile organisms, because they are subjected most uniformly to its power. In the case of plants, for example, the roots grow downward because they are positively geotropic, while the stems grow upward because they are negatively geotropic. The causes of posi-

${ }^{1}$ Cf. C. B. Davenport, Experimental Morphology, New York, I897, Vol. I, pp. 196-20r. 
tive geotropism seem more evident than those of negative geotropism. We do not need to go into a discussion of the question as to what is the nature of the force of gravity, but we know that it pulls matter towards the center of the earth. Consequently it does not appear surprising that organisms should be pulled downward. There has been a good deal of discussion as to how this takes place. ${ }^{1}$ Certain experiments seem to indicate that in cells whose contents are somewhat segregated from each other the heavier particles sink to the bottom. This seems to explain the positive geotropism of certain parts of sessile organisms, and, as we shall see, it may also explain the geotropic orientation of free-moving organisms.

But the causes of negative geotropism are much more obscure. It has been suggested that it is caused by the centrifugal force of the rotating earth. ${ }^{2}$ Certain experiments made by revolving organisms on a klinostat, a machine for testing the effect of the centrifugal force, indicate that this may explain negative geotropism in plants, though this is not certain. But it is doubtful if this can explain negative geotropism in free-moving organisms. In the case of many of these organisms it is evident that distinct orientation with respect to the line of gravity takes place. Various explanations have been suggested for both positive and negative geotropism in these cases. It has been suggested that it is due to the difference in pressure between the upper and the lower portions of the organism. Or it may be due to the fact that the different parts of the organism vary in their specific gravity so that the organism

${ }^{1}$ Loeb, The Dynamics of Living Matter, pp. 147-152; Davenport, Experimental Morphology, pp. II 2-II4.

${ }^{2}$ Loeb, Studies in General Physiology, p. 182; Davenport, op. cit., pp. 112-113. 


\section{Tropisms}

is oriented with its anterior end towards or away from the center of the earth. But the whole subject is so uncertain that it would hardly be worth while to discuss it further here.

In the higher organisms orientation to gravity seems to take place by means of certain mineral particles called otoliths in the auditory mechanism.

The reaction to gravity is not as strong in many cases as the reactions to other forces, so that it is frequently annulled by one or more of these other reactions. This, indeed, may happen to any of these reactions. An organism is almost always exposed to more than one of these forces, so that these forces are continually neutralizing and modifying each other. Consequently the behavior of an animal is the resultant of these forces and the responses of the organism to them. The geotropic response of an organism may change as a result of changes in its external circumstances or in its physiological state. The geotropic reactions seem to have functional value in many cases.

\section{CHeMotropism}

We will now turn to the reactions of organisms to chemical substances. By chemical substance is meant a substance which is diffusing itself in the surrounding medium; namely, the water or the air. Organisms are frequently oriented when they come near to these substances. Loeb explains such orientation as follows: "Theoretically we may assume that if substances diffuse in air or in water, the particles move in a straight line away from the center of diffusion. If they strike an organism whose surface is affected by the diffusing substances on one side only, the 
contractile protoplasm, or the muscles, turning the tip or the head or the whole organism toward that side, are thrown into a different state of contraction from their

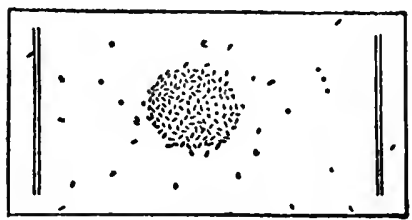

Fig. 2. - Positive chemotropism. Slide showing the positive reaction of paramecia to a drop of a weak solution of carbon dioxide. (After Holmes.) antagonists. The consequence is a turning or bending of the tip or the head until symmetrical points of the chemically sensitive surface of the body are struck by the lines of diffusion (or the diffusing particles) at the same angle. As soon as this occurs, the contractile elements on both sides of the organ, or organism, are in an equal state of contraction, and the animal will bend or move in the direction of the lines of diffusion." 1 He goes on to show that chemotropic orientation is not as definite usually as phototropic or geotropic orientation because the lines of diffusion are constantly being disturbed by variations in the temperature of the surrounding medium so that the lines of force are not regular.

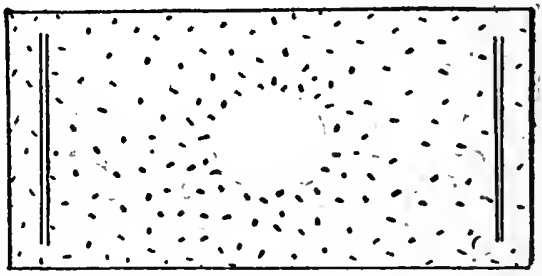

FIG. 3. - Negative chemotropism. Slide of paramecia four minutes after the introduction of a drop of $\frac{1}{2}$ per cent $\mathrm{NaCl}$. The drop remains empty. (After Jennings.)

Jennings ${ }^{2}$ explains the reaction of these lower organisms to chemical substances by means of the "avoiding reaction." That is to say, when an organism comes to a region in which

1 The Dynamics of Living Matter, pp. I52-153. See also Comparative Physiology of the Brain and Comparative Psychology, pp. 186-187.

2 Op. cit., p. 62. 


\section{Tropisms}

is diffusing a chemical substance towards which the organism is negatively chemotropic, it will give the avoiding reaction which will turn it in another direction. If the organism is positively chemotropic towards this substance, it will not react when entering the region of diffusion, but it will give the avoiding reaction when it comes to the boundary of the region, so that it will be held in this region. The degree of concentration of a solution which will produce a reaction varies greatly for different individuals, and also varies for the same individual as its sensitiveness changes.

Chemotropic reactions frequently have great functional value, especially for finding food and for mating. This is true, for example, of the insects. These reactions aid them in coming in contact with their food, in depositing their eggs upon matter which will serve as food for the young, and in bringing the sexes together for purposes of pairing. ${ }^{1}$ They have a similar utility for many other of the lower organisms. This chemical sense, as it is sometimes called, is the rudimentary form out of which differentiate the senses of smell and of taste. Very frequently the lower organisms locate their food by means of a chemical reaction. They are attracted to each other in similar fashion, and this frequently leads to conjugation, so that the reproductive process is stimulated. This tendency on the part of some of these organisms to come together has sometimes been called "sociability," but it is not, of course, the sociability displayed by the higher organisms. Jennings ${ }^{2}$ has described how paramecia tend to congregate together. Paramecium gives off carbon dioxide. If several of them

1 Cf. Loeb, The Dynamics of Living Matter, New York, Igo6, p. I55.

2 Op. cit., p. 68. 
chance to come together, a bubble of carbon dioxide may be formed. Each individual gives the avoiding reaction on coming to the edge of the bubble, so that the paramecia are held in the bubble. Other individuals on entering the bubble are prevented from leaving in similar fashion, so that in course of time the group may become very large.

\section{GaLVANOTROPISM}

The electric current causes certain reactions in the lower organisms which are usually grouped under the name of galvanotropism or electrotropism. Loeb explains galvanotropism as he does phototropism and geotropism. "When parallel current curves strike a symmetrical organ or organism sidewise, the contractile elements, e.g. muscles, on one side of the organ, or organism, undergo a higher degree of tension than on the other side; the outcome is a bending or turning of the organ or animal until its axis, or plane of symmetry, is in the direction of the current curves. As soon as this occurs, the symmetrical elements of the surface of the body are struck at the same angle by the current curves, and the kind and acceleration of chemical reaction is the same on both sides of the organism ; consequently the symmetrical muscle elements show the same state of contraction. But the fact that the current curves penetrate throughout the whole animal causes often complications which prevent an ideal orientation such as we observe in the case of light." 1 The electric current frequently does injury to these organisms. This is probably due to the fact that they are rarely ever subjected to it in nature, so that they have not become adapted to it.

1 The Dynamics of Living Matter, p. I4I. 


\section{STEREOTROPISM}

Another group of reactions is that of reactions to contact. These are sometimes grouped under the term stereotropism and also under the term thigmotropism. Verworn considers thigmotropism, or thigmotaxis as he calls it, as a form of barotaxis, under which term he groups all reactions which result from changes in pressure relations. "All mechanical stimulation of living substance consists in a change of the pressure relations under which it exists. Every degree of pressure can act as a stimulus, from crushing or cutting, which destroys the continuity of the substance, down to the slightest touch and the most delicate change in the pressure of the air or the water that surrounds the organism." 1 Under thigmotaxis he groups all reactions which result from the contact of living substance with bodies which are more solid than it.

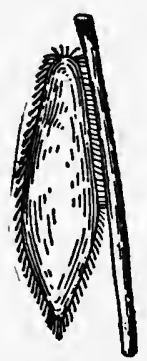

Fig. 4.-Stereotropism, or contact irritability. Paramecium at rest against a cotton fiber, showing the motionless cilia in contact with the fiber. (After Jennings.)

Davenport regards thigmotaxis, or contact irritability, as it is perhaps more appropriately called by some writers, as the effect of molar agents acting upon organisms. He explains the effect of contact upon organisms as follows: "In many non-living substances, especially organic compounds, violent chemical changes (explosions) are brought about by contact and especially by repeated vibrations. So, too, in protoplasm, chemical change, exhibiting itself in modified metabolism, frequently follows contact. The 


\section{The Science of Human Behavior}

explanation adapted to the non-living series of phenomena is adapted to the living series also, - the molecules of the substance are complex, loosely associated, very unstable, so that even a slight mechanical disturbance will serve to dissociate their atoms." 1 It appears from this passage that contact causes a chemical change in the organism. The term contact irritability therefore seems to be the best one to apply to these reactions. In any case there certainly is no tropism involved in these reactions, for there are no lines of force which cause them in the organisms. It is therefore misleading to group these reactions under such a name as stereotropism or thigmotropism.

The functional value of contact irritability is similar to that of chemotropism, because it aids greatly in securing food and in causing reproduction. In many cases after chemotropism has brought the organism to food, it is contact irritability which leads it to grasp it and to ingest it. After chemotropism has brought two individuals close together, it usually is contact irritability which forces them to conjugate. This is also true of the higher organisms, for contact irritability seems to play a part very frequently, perhaps always, in bringing about copulation.

\section{RHEOTROPISM}

Another form of reaction is reaction to a current of water, sometimes called rheotropism or rheotaxis. Inasmuch as this is a reaction to a pressure stimulus, Verworn calls this a form of barotaxis. ${ }^{2}$ Davenport regards it as a form of thigmotropism. "We now turn to the consideration of Rheotaxis, which may be regarded provisionally as a form 


\section{Tropisms}

of thigmotaxis, although the possibility of its being rather a case of chemotaxis is not to be excluded." 1 He does not indicate why rheotropism may be a form of chemotropism. Jennings also speaks of rheotropism as if it was a form of stereotropism. "The reaction is essentially a response to a mechanical disturbance, comparable to that due to the touch of a solid body." 2

Rheotropism is manifested frequently by higher organisms, such as fishes which tend to head upstream. But it has been questioned whether there is any tropism involved in this reaction, and the investigations of Lyon seem to indicate that it is simply an optical reflex and that it is an effort upon the part of the organism to keep itself in such a position that it shall appear to its sense of vision that it is at rest. ${ }^{3}$ It may also be that rheotropism like stereotropism is not a real tropism for the lower organism as well, for there are no lines of force acting upon the organism. The current of water is simply a molar agent pressing upon the organism.

Rheotropism probably has functional value in a good many cases, though this subject has not been investigated very much as yet. It appears that it plays a part in bringing about fertilization in the human species, as is indicated in the following passage from Verworn: "It is easy to assume that the human spermatozoa are rheotactic and find their way to the egg cell by means of this property. When the spermatozoa come into the uterus, they meet a current of mucous liquid coming toward them, since the cilia of the epithelium lining the uterine cavity have a

1 Op. cit., p. 108.

${ }^{2}$ Op. cit., p. 74 .

3 E. P. Lyon, Am. Jour. of Physiology, Vol. XII, p. I49 (I904). 
direction of stroke toward the $o s$, and hence produce a current toward the outside. That it is chemotaxis of the spermatozoa toward the ovum which points out the path to them becomes very improbable when it is remembered that the spermatozoa wander upward in the uterus before the ovum has left the ovarian follicle." 1 It is probably useful also to fish because it leads them to go up streams, where they can lay their eggs in quiet and safe places. ${ }^{2}$

\section{OTHER TROPISMS}

A reaction similar to rheotropism is the reaction to a current of air, usually called anemotropism..$^{3}$ It manifests itself usually in winged organisms, and practically everything which has been said about rheotropism is true also for anemotropism.

The lower organisms react sometimes in certain more or less definite ways to heat. These reactions may be grouped under the term thermotropism or thermotaxis. They seem to be caused by differences in the intensity of the heat at different points on the organism, thus causing unequal stimulation. ${ }^{4}$

There are a number of other kinds of reactions which are similar to the reactions to heat, such as hydrotropism or hydrotaxis, ${ }^{5}$ which are reactions caused by differences in moisture, and tonotropism or tonotaxis, ${ }^{6}$ which are reactions to differences in the density of the surrounding medium. These reactions have not been investigated very much as yet, and there is not the space to discuss them further here.

Op. cit., p. 445 . 2 Cf. Davenport, op. cit., p. rog.

${ }^{3}$ Cf. Washburn, op. cit., pp. 154, 188-189, 288.

4 Cf. Davenport, op. cit., p. 262 ; Verworn, op. cit., p. 45 I.

Cf. Davenport, op. cit., p. 66.

${ }^{6}$ Cf. Davenport, op. cil., p. 89. 


\section{CHAPTER VII}

\section{THE EVOLUTION OF ANIMAL BEHAVIOR}

Determination of behavior by form and past experience and by external stimuli, II7. - Description of the behavior of amœba, paramecium, stentor, etc., II8. - The action system, I26. - The reflex, 128. - The physiological state of the organism as a factor in the determination of behavior, I3I. - The regulation of behavior, I32. - The development of behavior in the race, 133. - Organic selection, 134. - The laws of animal behavior, 135 .

IN the preceding chapter have been reviewed briefly the reactions of organisms to the external forces or stimuli enumerated in the classifications of Davenport and Verworn which have been cited. So far as I know, all the investigators of animal behavior are agreed that the actions of organisms are determined in the last analysis and in the long run by external forces. That is to say, the forms of these organisms have been determined in the past by these external forces. Furthermore, their experience. in the past has been determined by their forms and by external forces. So that inasmuch as the behavior of an organism at any moment is not determined entirely by the immediate stimulus, but in part by its form and by its past experience, the behavior as a whole can in the last analysis be traced back to external forces. I wish therefore to discuss next the part played by the forms and past experience of the organisms themselves in determining the reactions of the lower animals. 


\section{The Determination of Behavior}

There has been a good deal of difference of opinion among the investigators of the behavior of the lower animals as to the extent to which this behavior is being determined by form and past experience and the extent to which it is being determined by the immediate stimulus. This difference of opinion has manifested itself to the greatest extent in the discussion of the tropism theory. It has, perhaps, been to a certain extent true that the more ardent supporters of the tropism theory have tended to minimize the part played by the organism in determining its behavior, while those who have opposed the theory or who have not made much use of it have tended to emphasize the part played by the organism in determining its behavior. This last has been somewhat true of Jennings, who in the numerous papers he has published on the subject and especially in his able work on the "Behavior of the Lower Organisms," which has already been cited several times in previous chapters, has made a careful and detailed study of the causes of the behavior of lower organisms. I sha 1 therefore review briefly the investigations and theories of Jennings in this field.

Jennings devotes more than two thirds of his principal work to an account of investigations made by him in the study of the behavior of several species of protozoa and of the lower metazoa. The first species he studies is the Amœba, which is one of the lowest organisms. The Amœba is a shapeless mass of jellylike protoplasm which has almost no structure to determine its reactions. After describing various experiments in which it was subjected to different stimuli, he sums up the results as follows: "We 
find that the simple naked mass of protoplasm reacts to all classes of stimuli to which higher animals react (if we consider auditory stimulation merely a special case of mechanical stimulation). Mechanical stimuli, chemical stimuli, temperature differences, light, and electricity control the direction of movement, as they do in higher animals. In

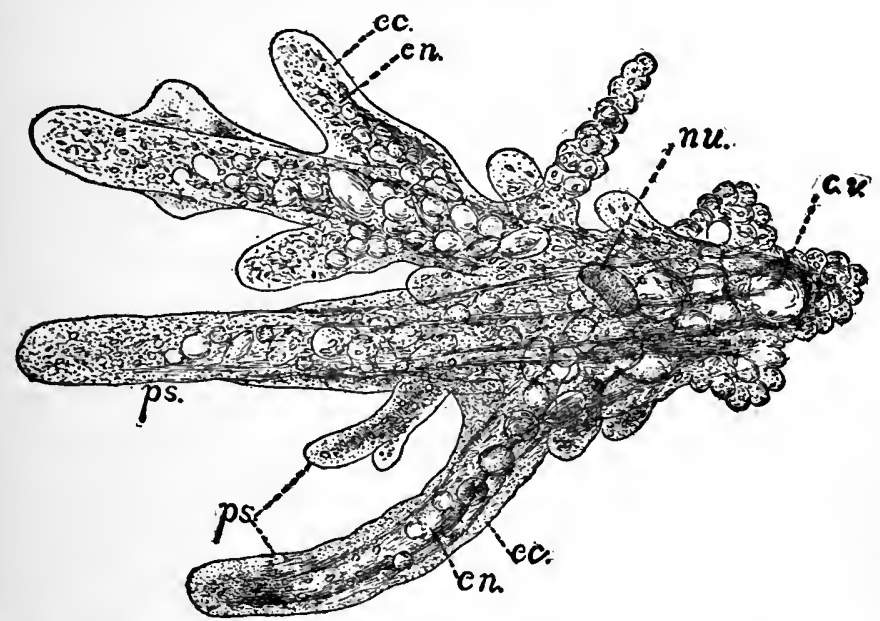

Fic. 5. - Amerba proteus. c.v., contractile vacuole; ec., ectosarc; en., endosarc; $n u$. ., nucleus; ps., pseudopodia. (From Jennings, after Leidy, slightly modified.)

other words, Amœba has some method of responding to all the chief classes of life conditions which it meets." $1 \mathrm{He}$ goes on to show that the behavior of Amœba under any given conditions is not determined entirely by the immediate stimuli, but that behavior in the past is influencing present action. He distinguishes three main types of behavior, which he calls the positive reaction, the negative or avoiding reaction, and the food reaction. An Amœba may respond in any one of these three ways to a given stimulus. 
What determines in which way it will respond, and will it always respond in the same way to such a stimulus? Jennings discusses these questions as follows: "What decides whether the reaction to a given stimulus shall be positive or negative? This question touches the fundamental problem of behavior. The nature of the physical or chemical action of an agent does not alone determine the reaction, for to the same agent opposite reactions may be given, depending on its intensity, or upon various attendant circumstances. If we should make a chemical or physical classification of the agents affecting movement in Amœba, this would not coincide with a classification based on the reactions given. But the agents which produce a negative reaction are in general those which injure the organism in one way or another, while those inducing the positive reaction are beneficial. Any agent which directly injures the animal, such as strong chemicals, heat, mechanical impact; produces the negative reaction. The positive reaction is known to be produced only by agents which are beneficial to the organism. It aids the animal to find solid objects on which it can move, and is the chief factor in obtaining food. Thus the behavior of Amœba is directly adaptive; it tends to preserve the life of the animal and to aid it in carrying on its normal activities." 1 How the behavior of Amœba has become adaptive, Jennings does not explain at this point, but as we shall see he takes up this subject later on.

The next group of organisms he studies are certain species of bacteria. These are the lowest organisms which have a definite form and special organs of locomotion. It is hard

$$
1 \text { Op. cit., p. } 23 .
$$


to determine whether they are animals or plants, though they are usually regarded as plants. Jennings makes a special study of their reactions to food. He finds that their reactions are determined to a large extent by the needs of the metabolic process. "A relation of great significance becomes evident on examining the facts; behavior under stimulation depends on the nature of the normal life processes, - especially the metabolic processes. Bacteria that require oxygen in their metabolism collect in water containing oxygen; bacteria to which oxygen is useless or harmful avoid oxygen. Bacteria that use hydrogen sulphide in their metabolism gather in that substance. Bacteria that require light for the proper performance of their metabolic processes gather in light, while others do not." 1 He sums up these reactions as follows: "Behavior that results in interference with the normal metabolic processes is changed, the movement being reversed, while behavior that does not result in interference or that favors the metabolic processes is continued." 2 Thus we see that the behavior of bacteria also is adaptive and regulatory. The negative reaction is used to avoid those things which are harmful, while the positive reaction is used to secure those things which are beneficial. He is, however, careful to point out that the adaptation is not always perfect.

Next he studies Paramecium, a species of infusoria. Paramecium is a cigar-shaped, unicellular animal which propels itself by means of cilia. It is not bilaterally symmetrical, but slightly spiral in form, because it has a broad, oblique groove on one side, the oral surface, called the oral groove, or peristome. The cilia in the oral groove

$$
{ }^{1} \text { Op. cit., p. } 39 . \quad{ }^{2} \text { Op. cit., p. } 39 .
$$


make stronger strokes than the others so the head is constantly being turned away from the oral side. But at the

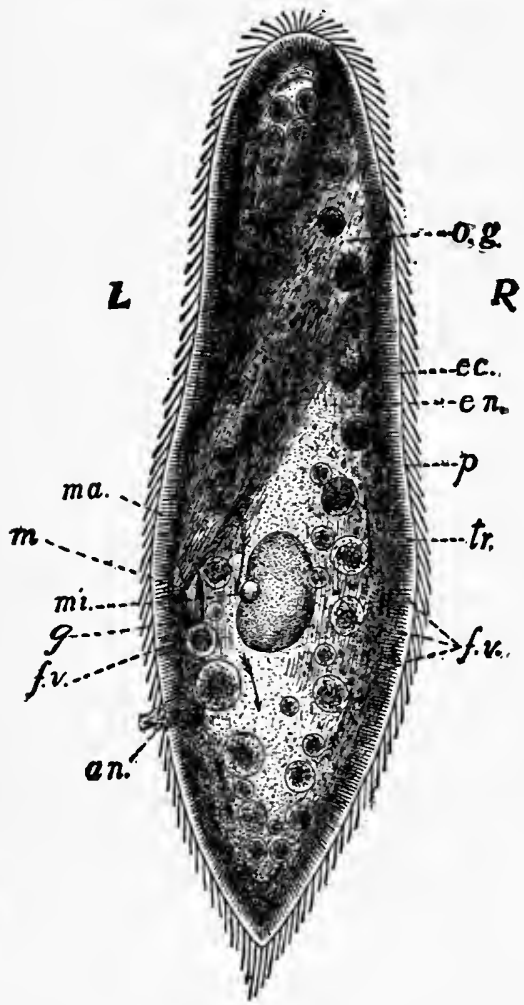
face. $L$, left side; $R$, right side; $a n$., anus; $e c$. , ectosarc ; $e n$. , endosarc; f.v., food vacuoles; g., gullet ; m., mouth ; ma., macronucleus ; $m i$., micronucleus; $0 . g$., oral groove; $p$., pellicle; tr., trichocyst layer. The arrows show the direction of movement of the food vacuoles. (After Jennings.)
Fig. 6. - Paramecium, viewed from the oral sur-

same time the animal is rotating on its long axis, because the cilia strike, not directly backward, but obliquely to the right, so that the animal rolls over to the left. Consequently the tendency to move towards the aboral side is compensated and the animal moves forward in a spiral course. This spiral course is useful to it in giving the avoiding reaction. If it strikes an obstacle in its path, its cilia reverse their action and it moves backward a short distance. Then as it starts forward again on its spiral course it will be moving in a somewhat different direction and may avoid the obstacle. If it should hit it again, however, it will repeat the avoiding reaction and will continue to do so until it has passed the obstacle. "Paramecia 
react to most of the different classes of stimuli which act upon them, in the way just described. Mechanical stimuli, such as solid obstacles, or disturbances in the water; chemicals of all sorts; heat and cold; light that is sufficiently powerful to be injurious; electric shocks, and certain disturbances induced by gravity and by centrifugal force, all cause the animal to respond by the avoiding reaction, so that it escapes if possible from the region or condition that acts as a stimulus." 1

But, according to Jennings, Paramecium gives a positive reaction also. Upon a little analysis, however, this positive reaction seems to me to be nothing more than the negative or avoiding reaction given in such a fashion as to keep the animal under a certain stimulus which usually is beneficial to it. For example, a Paramecium may swim into a region in which a chemical is dissolved to which it does not give the negative reaction. Upon and after entering this region it will make no change of movement. But when it comes to the boundary of this region it will give the avoiding reaction and will thus be kept within this region. Just why the negative reaction is given when the boundary of this region is reached is not explained, but it is contended that both the negative and the so-called positive reactions are usually beneficial to the organism, so that they must be adaptive and regulatory. "We may sum up the usual behavior of Paramecium under the various stimuli of the environment in the following way. The natural condition of the animal is movement. In constant external conditions (unless destructive) the movements are not changed, - that is, there is no reaction, - even though these con-

$$
1 \text { Op. cit., p. } 5 \text { r. }
$$




\section{The Science of Human Behavior}

ditions do not represent the optimum. But as its movements carry the animal from one region to another, the environmental conditions affecting it are of course changed, and some of these changes in condition act as stimuli, causing the animal to change its movements. If the environmental change leads towards the optimum, there is no reaction, but the existing behavior is continued. To a change leading away from the optimum (in either a plus or minus direction), Paramecium responds by the 'avoiding reaction.' This consists essentially in a return to a previous position, through a backward movement, then in 'trying' different directions of movement till one is found which leads toward the optimum." 1 In this case, as in the previous ones, he reserves the discussion of how the behavior of this organism became adaptive for a later part of his book.

So far I have spoken only of cases in which there is but one stimulus acting upon Paramecium. But under ordinary conditions it is subjected to a combination of stimuli, so that its behavior is determined by all of these stimuli. Furthermore, its behavior is undoubtedly influenced by internal changes. It is difficult to study the physiological condition of so minute an animal as Paramecium, but there is good reason to believe that it is characterized at different times by physiological states which correspond to fatigue, hunger, acclimatization, etc. These states have, of course, been caused in part by past stimuli, so that here we have rudimentary examples of the modification of behavior through the past experiences of the animal.

Further on Jennings gives fuller illustrations of such modifications in the case of Stentor reselii. This is a trumpet-shaped, unicellular

1 Op. cit., pp. 57-58. 


\section{The Evolution of Animal Behavior 125}

animal which is usually attached by its foot to a water plant or piece of débris. If some carmine is added to the water around it, the changes in its behavior which result are summed up as follows :-

"I. No-reaction at first: the organism continues its normal activities for a short time.

" 2 . Then a slight reaction by turning into a new position, - a seeming attempt to keep up the normal activities and yet get rid of the stimulation.

" 3 . If this is unsuccessful, we have next a slight interruption of the normal activities, in a momentary reversal of the ciliary current, tending to get rid of the source of stimulation.

" 4 . If the stimulus still persists, the animal breaks off its normal activity completely by contracting strongly - devoting itself entirely, as it were, to getting rid of the stimulation, though retaining the possibility of resuming its normal activity in the same place at any moment.

"5. Finally, if all these reactions remain ineffective, the animal not only gives up completely its usual activities, but puts in operation another set, having a much more radical effect in separating the animal from the stimulating agent. It abandons its tube, swims away, and forms another one in a situation where the stimulus does not work upon it." 1

These changes in behavior therefore take place while the organism is under the same stimulus, so that they must be caused by changes in

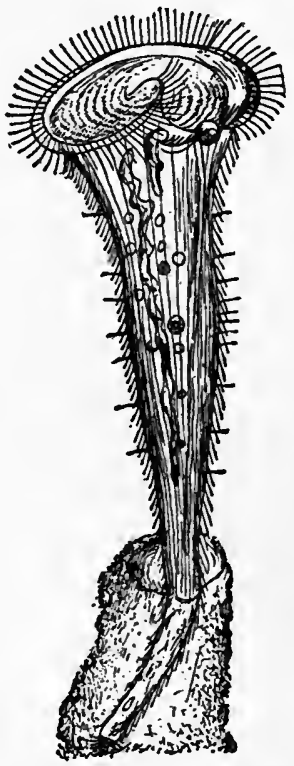

Fig. 7.-Stentor reselii Ehr. (From Jennings, after Stein.) the organism itself. The organism appears to be "trying" different methods of getting rid of a stimulus which is obnoxious to it. Consequently its behavior in this respect is singularly like that of higher organisms, and the similarities and differences between the behavior of the unicellular and the higher organisms are indicated in the following passage: "The same individual does not always behave in the same way under the same external conditions, but the behavior depends upon the physiological condition of the animal. The reac- 
tion to any given stimulus is modified by the past experience of the animal, and the modifications are regulatory, not haphazard, in character. The phenomena are thus similar to those shown in the 'learning' of higher organisms, save that the modifications depend upon less complex relations and last a shorter time." 1

\section{The Action System}

It must be evident that the structure of a given organism must limit the number and kinds of actions which that organism can perform. There are, in the first place, for each organism a number of simple movements and then a larger-number of combinations of these simple movements. The whole set of these actions taken together Jennings calls the action system. For example, he describes the action system of Paramecium as follows: "The action system of Paramecium is based chiefly on the spiral course, with its three factors of forward movement, revolution on the long axis, and swerving toward the aboral side. The behavior under most conditions is determined by variations in these three factors. Such variations, combined in a typical manner, produce what we have called the avoiding reaction." 2

Among the higher organisms we do not expect to find an animal without wings flying nor an animal without feet walking. So among the lower organisms behavior is limited and determined to a large extent by structure. For example, the infusoria can be divided according to their behavior, which is determined in large part by structure, into those that are sessile, those that creep over surfaces, and those that swim freely. "The behavior is simplest and least varied in the free-swimming organisms; more 
varied in those which habitually creep along a surface; most complex in those which live attached. The reason for this seems to be as follows: In the open water the conditions are exceedingly simple. The free-swimming organism may escape an injurious stimulus simply by swimming away. In the fixed organism, on the other hand, the conditions are more complex. At any moment both the solid and the free liquid are acting on the organism. For a fixed animal to obtain food and escape injurious conditions, varied devices are necessary. It cannot at once solve any difficulty by departing, as the free organism can. We find, then, that such fixed organisms have developed varied reaction methods." 1 We have already illustrated these differences in the case of a free-swimming organism like Paramecium, whose reactions are very simple, and of an organism like. Stentor roeselii, which is sessile most of the time, but creeps over surfaces sometimes, and whose reactions are much more varied than those of Paramecium.

Jennings next describes studies he has made in the behavior of certain species of the lower metazoa. I have not the space to summarize these studies, though they are of the greatest interest. We can only note his general conclusions. There are two great differences between the metazoa and the protozoa. The metazoa are multicellular, while the protozoa are unicellular; the metazoa have a nervous system, while the protozoa do not have one. On account of these differences it has been thought by many that the differences between the behavior of the protozoa and of the lower metazoa must be very great. But Jennings comes to a different conclusion. After making a detailed 
comparison of the behavior of these two groups of organisms he expresses himself as follows: "All together, there is no evidence of the existence of differences of fundamental character between the behavior of the Protozoa and that of the lower Metazoa. The study of behavior lends no support to the view that the life activities are of an essentially different character in the Protozoa and the Metazoa. The behavior of the Protozoa appears to be no more and no less machinelike than that of the Metazoa; similar principles govern both." 1 With respect to the nervous system he comes to the same conclusion as Loeb; namely, that "we do not find in the nervous system specific qualities not found elsewhere in protoplasmic structures," so that most of the activities which have usually been considered peculiar to the nervous system can be demonstrated in the protozoa, in which there is no nervous system. This is a subject which will be discussed in the next chapter on the nervous system.

Several times in the course of this exposition of Jennings; work it has been stated that he explains in the latter part of his book how the behavior of these lower organisms becomes adaptive and regulatory. I will now summarize this explanation and his general theory as to the behavior of the lower organisms.

\section{The Reflex}

He commences with a discussion of the nature of reflex action. By reflex action is frequently meant the contraction of a muscle when a certain nerve is stimulated. It is called reflex because the stimulation is supposed to pass

$$
1 \text { Op. cit., p. } 263 .
$$


along the sensory nerve to the central nervous system and is then reflected back to a muscle. It is evident that for this sort of reflex action a nervous system is needed, and this kind of reflex action will be discussed in the next chapter. But the term reflex action is sometimes applied to any case of an invariable reaction to a given stimulus. In this sense it is simply a type of action without regard to the existence of a nervous system, and it is in this sense that Jennings uses the term. It is believed by some that the reactions of the lower organisms are reflex in this sense, that they are all invariable. But Jennings denies this on the ground that the same stimulus will not necessarily produce the same reaction invariably, because changes in the physiological state of the organism may cause variations in the reaction. "If, then, we consider the reflex an invariable reaction to a given stimulus, we cannot hold that behavior in lower organisms is made up of reflexes. Indeed, the fact that stands out most clearly in the behavior is the following: Each stimulus causes as a rule not merely a single definite action that may be called a reflex, but a series of 'trial' movements, of the most diverse character, and including at times practically all the movements of which the animal is capable. The reaction to a given stimulus depends on the physiological state of the organism, not alone on its anatomical structure; and physiological states are variable. This is true both for the infusoria and for man." 1

This tendency to characterize the behavior of the lower organisms as purely reflex arises out of the desire to explain this behavior on mechanical grounds without assuming

$$
1 \text { Op. cit., p. } 280 \text {. }
$$


the existence of consciousness. This is, I believe, a laudable desire. But just because the behavior is not invariable, we do not have to assume that consciousness exists and that therefore the behavior is not purely mechanical. The physiological state of the organism may be regarded as a part of its mechanical structure, so that when we explain variations in its behavior by changes in its physiological state, we are still explaining its behavior on mechanical grounds. But the behavior of higher organisms also can be explained in similar fashion on mechanical grounds, so that no distinction in this respect can be made between the behavior of the higher and the lower organisms. If, then, we are to regard reflex action as invariable reaction to a given stimulus, no species is characterized with reflex action. We may, however, give a different meaning to the term and mean simply that the behavior of an organism is determined by its physical and chemical characteristics. It is evident that in this sense the behavior of every species of organism is reflex, and consequently no distinction can be made between the higher and the lower species. "What a given organism does under stimulation is limited by its action system, and within these limits is determined largely by its physiological condition at the time stimulation occurs. In the lowest organism the action system confines the variations in behavior within rather narrow limits, and the different physiological conditions distinguishable are few in number; hence the behavior is less varied than in higher animals. But the difference is one of degree, not of kind." 1

1 Op. cit., p. $28 \mathrm{r}$. 


\section{The Physrological State}

Since we must take the physiological state into consideration in studying the behavior of an organism, we must consider what determines the physiological state. In the first place, the physiological state of an organism may be changed by progressive internal processes, especially those of metabolism. These changes are governed by the laws of metabolism. In the second place, the physiological state of an organism may be changed by the action of external forces. From the study of these changes, Jennings deduces what he calls the law of the resolution of physiological states, which he regards of the greatest importance in explaining the behavior of organisms. He states this law as follows: "The resolution of one physiological state into another becomes easier and more rapid after it has taken place a number of times." 1 This law is based upon the fact that a physiological state is a dynamic condition, not a static one. "It is a certain way in which bodily processes are taking place, and tends directly to the production of some change. - The changes toward which the physiological state tends are of two kinds. First, the physiological state (like the idea) tends to produce movement. This movement often results in such a change of conditions as destroys the physiological state under consideration. But in case it does not, then the second tendency of the physiological state shows itself. It tends to resolve itself into another and different state. Condition I passes to condition 2, and this again to condition 3. This tendency shows itself even when the external conditions remain uniform." ${ }^{2}$ 
The law which has been cited is based upon this second tendency and indicates that the resolution of one physiological state into another takes place more readily the more frequently it is repeated. Sometimes where a number of states follow each other in a series, the resolution of one into the other may come to take place so rapidly as to be instantaneous, so that only the last state determines what the form of behavior is to be. Jennings believes that this law explains in large part what are called memory, association, habit formation, and learning in the higher organisms. But he believes that it applies to the lower organisms as well, and that the only differences between organisms is as to the readiness with which the quick resolution of one physiological state into another becomes established and the extent to which such a habit is permanent. A little further on he shows that the adaptive and regulatory character of behavior grows in large part out of the working of this law.

He then classifies the factors upon which behavior depends under the following heads:-

"I. The present external stimulus.

"2. Former stimuli.

"3. Former reactions of the organism.

"4. Progressive internal changes (due to metabolic processes, etc.).

"5. The laws of the resolution of physiological states one into another." 1

He now states a principle which he says is of the greatest importance for the understanding of behavior. It would seem as if this principle should have been introduced much earlier in the book, for it throws light on the "method of 
trial" which he has already discussed. This is the principle which has sometimes been called the "selection of overproduced movements," but which Jennings prefers to call the "selection of the proper conditions of the environment through varied movements." He describes the phenomena from which this principle is deduced as follows: "The stimulus does not produce directly a single simple movement (a reflex act) of a character that relieves the organism at once from the stimulating condition. On the contrary, stimulation is followed by many and varied movements, from which the successful motion is selected by the fact that it is successful in causing cessation of stimulation." 1

\section{DeVelopment OF BeHAVIOR}

He devotes one chapter to the discussion of the development of behavior in the individual and in the race. Enough has been said to illustrate the development of behavior in the individual, but a word should be said about its development in the race. Jennings believes that in unicellular species forms of behavior acquired by individuals may be transmitted directly to their offspring, because in these species there is no distinction between germ cells and body cells, so that all acquired characters belong to the germ cells. But the situation is very different in multicellular organisms, for in them acquired characters are rarely if ever transmitted from parents to offspring. Consequently, if forms of behavior are to become racial as well as individual, it must be through some other means. Jennings believes that the development of racial forms of behavior has come about through the natural selection of congenital variations and

1 Op. cit., p. 302. 


\section{The Science of Human Behavior}

especially through that form of natural selection which has been called organic selection by J. Mark Baldwin, Lloyd Morgan, and H. F. Osborn. The theory of organic selection ${ }^{1}$ was formulated in reply to certain criticisms of the theory of natural selection. It was contended that the theory of natural selection did not provide for a series of variations along a definite line of evolution, because the variations appear purely by chance, and the selection of them also is purely by chance. According to the theory of organic selection, the selection of these chance variations is guided and determined in part by characters acquired by individuals. That is to say, variations will appear by chance which are beneficial to the individual, but which are not sufficiently great to be decisive in the struggle for existence. These variations are shielded and conserved by individual accommodations until they are sufficiently great to be selected for their own sake. This theory has been much criticized, but Jennings apparently thinks it sound. As applied to behavior, he puts it as follows: "Thus individual selection and natural selection necessarily work to the same result. One selects from among the different acts of the same individual, the other from among those of different individuals. The thing selected is the same in each case, - namely, the adaptive reaction." 2

In the following chapter he discusses the relation of behavior in lower organisms to psychic behavior. Inasmuch as psychic behavior is to be discussed in a later chapter on consciousness I shall not dwell upon the matter here, except to say that he points out phenomena in the behavior

\footnotetext{
1 Baldwin gives a good exposition of this theory and its history in Development and Evolution, New York, 1902.

2 Op. cit., p. 325.
} 
of these lower organisms which seem to be analogous to such psychic characteristics as perception, discrimination, choice, attention, etc., in higher organisms.

In the last chapter he points out again, as he already has done at the beginning of the book, that behavior illustrates only one form of regulation and that regulation exists in the other processes of the organism. "Behavior is merely a collective name for the most obvious and most easily studied of the processes of the organism, and it is clear that these processes are closely connected with, and are indeed outgrowths from, the more recondite internal processes. There is no reason for supposing them to follow laws different from those of the other life processes, or for holding that regulation in behavior is of a different character from that found elsewhere." 1

This review of Jennings can be closed best by giving his final summary of the fundamental features of the method of individual regulation. "The organism is a complex of many processes, of chemical change, of growth, and of movement; these are proceeding with a certain energy. These processes depend for their unimpeded course on their relations to each other and on the relations to the environment which the processes themselves bring about. When any of these processes are blocked or disturbed, through a change in the relations to each other or the environment, the energy overflows in other directions, producing varied changes, - in movement, and apparently in chemical and growth processes. These changes of course vary the relations of the processes to each other and to the environment; some of the conditions thus reached relieve the in- 
terference which was the cause of the change. Thereupon the changes cease, since there is no further cause for them; the relieving condition is therefore maintained. After repetition of this course of events, the process which leads to relief is reached more directly, as a result of the law of the readier resolution of physiological states after repetition. Thus are produced finally the stereotyped changes often resulting from stimulation. This method of regulation is clearly seen in behavior, where its operation is, in the later stages, what is called intelligence. Its application to chemical and form regulation is at present hypothetical, but appears possible." 1

In an earlier chapter it was shown how difficult it is to determine the relative importance of external and internal forces in organic evolution. It is now evident that the same thing is true in the study of animal behavior. This is illustrated in the writings of two of the investigators whose work has been discussed. We have seen that Loeb emphasizes the part played by external forces in the determination of behavior, while Jennings emphasizes the complexity of even the lowest organisms and the consequently important part played by internal forces. Further research will throw more light upon the relative importance of these two sets of forces.

We have seen that the typical form of behavior for the lowest organisms without a nervous system is the tropism, meaning by that term any direct response of an organism to an external force. In the succeeding chapters will be discussed the more indirect and complex forms of behavior. 


\section{CHAPTER VIII}

\section{THE EVOLUTION OF THE NERVOUS SYSTEM AND THE REFLEX ACTION}

Progressive increase in the self-determination of behavior, I37. - Nerve cells and fibers, I37. - The specific qualities of nervous matter, 138. - Definitions of the reflex action, I40. - Receptor, adjustor, and effector organs, I43. - The simple reflex action, I44. - The conductibility of nervous matter, I45. - The adaptive character of reflex actions, 146. - The origin of the nervous system, I47. - The appropriation of effectors by the nervous system, I49.The selective excitability of receptor organs, I50. - The neurone, I54. - The connections between the neurones, 155 . - The central nervous system, 156 .

IN the last three chapters have been discussed the direct reactions of the lower organisms to external forces. We found that the behavior even of these lower organisms is to a certain extent self-determined; that is to say, it is determined in part by the structure of the organism and by forces which originate within the organism. Such selfdetermination of behavior increases as we go up in the organic scale. In animals this is in large part due to the evolution of the nervous system, so that the peculiar features of the behavior of the higher animals must be attributed principally to this fact. We must therefore trace the evolution of the nervous system in order to understand the behavior of the higher animals, and especially of man.

\section{Specific Qualities of Nervous Matter}

The nervous system consists of nerve cells and fibers which extend all over the body and which influence the behavior 
of the organism by determining the actions of the muscles and glands with which they are connected. The functions performed by the nervous system are so peculiar to itself that it has been the tendency of some to draw a sharp line of distinction between the nervous system and the rest of the organism. But others have contended that nervous substance is nothing more than a somewhat specialized form of protoplasm. For example, Loeb says that "irritability and conductibility" are the only qualities essential to nervous reactions and that "these are both common qualities of all protoplasm." 1 According to him, the only difference between ordinary protoplasm and nervous substance is that nerves "are quicker and more sensitive conductors than undifferentiated protoplasm." 2

Jennings also is inclined to take this view. After reviewing the similarities between the behavior of animals with and those without a nervous system, he speaks as follows: "A comparison of the behavior of the Protozoa with that of the lower Metazoa lends powerful support to that view of the functions of the nervous system which is so ably maintained by Loeb in his brilliant work on 'The Comparative Physiology of the Brain and Comparative Psychology.' According to this view we do not find in the nervous system specific qualities not found elsewhere in protoplasmic structures. The qualities of the nervous system are the general qualities of protoplasm. Certain of these general qualities have become much accentuated in the protoplasm of the nervous system, while in the remainder of the protoplasm of the metazoan body they are less

${ }^{1}$ Comparative Physiology of the Brain and Comparative Psychology, New York, I900, p. 5 .

2 Op. cit., p. 6. 


\section{The Evolution of the Nervous System 139}

strongly marked, being partially obscured by differentiations in other directions." 1

Looking at this question from the evolutionary point of view, it is inconceivable that there is any absolute distinction between the nervous system and the rest of the organism, because we know that all parts of the organism have a common origin and that all of them have differentiated from the primordial protoplasm. It is not surprising that writers like Loeb and Jennings, who have been leaders in the investigation of the behavior of the lower organisms, should minimize the differences between the animals without and those with a nervous system, for they are familiar with the species in which the nervous system is in a very rudimentary state, so that the differences in behavior between these species and those without a nervous system cannot be very great. But in the higher animals the nervous system, because of its specialized powers of conductibility, integrates a complex organism in a way which would be utterly impossible without a nervous system. So that while nervous matter is in the same evolutionary order with the other parts of the organism, and while we may agree with Jennings that most if not all of the features of behavior in the animals with a nervous system are to be found in a rudimentary form in the species without a nervous system, yet we must bear in mind that such behavior becomes far more complex in the higher animals and that most of the psychic phenomena which characterize the higher species would have been impossible without a nervous system.

It will be impossible in this chapter to give an extended account of the evolution of the nervous system or a detailed

${ }^{1}$ Behavior of the Lower Organisms, New York, 1906, pp. 263-264. 


\section{The Science of Human Behavior}

description of it as it now exists in the higher animals. Such a description is to be found in any treatise on neurology, and there is some literature on the evolution of the nervous system, though this literature is still somewhat limited. But the more important points, which will help us to understand the behavior of the higher animals, will be discussed.

\section{The Reflex Action}

Most discussions of the nervous system commence with the reflex action. The reason for this is that many writers on the subject regard the reflex as the fundamental type or unit of nervous action. For example, McDougall says that the simple reflex is "the fundamental type of all nervous action." 1 Parker says that "the physiological unit in the operations of the nervous system is the reflex." 2 But as we have already noted in the last chapter, this term has two meanings, and we must make clear just what we mean by it. Most of the writers who define reflex action indicate the two meanings. For example, Hobhouse speaks as follows of the term: "The most primitive form in which response is adapted to requirements is that in which a simple sensory stimulus calls forth a uniform reaction on the part of the organism. Such a response is known as Reflex action." But he goes on immediately to say: "This is an extended usage, and etymologically is, it must be admitted, not altogether appropriate. The term applies strictly to animals with a developed nervous system. In such animals the reflex act consists of two distinct move-

1 W. McDougall, Physiological Psychology, London, 1905, p. 15.

2 G. H. Parker, The Origin of the Neroous System and its Appropriation of Effectors, in the Popular Science Monthly, Vol. LXXV (1909), p. 56. 


\section{The Evolution of the Nervous System 141}

ments or processes, a sensory or afferent process, and a motor or efferent process." 1 Loeb speaks at first of a reflex as follows: "A reflex is a reaction which is caused by an external stimulus, and which results in a coördinated movement." 2 But a little further on he says: "This passage from the stimulated part to the central nervous system, and back again to the peripheral muscles, is called a reflex." 3 It is evident that the first statement involves a broader meaning than the second one, unless he means to imply that a coördinated movement is impossible without a nervous system.

It is evident in the case of all these writers that each one of them first defines reflex action as being a broad type of reaction which does not require a nervous system, and then defines it more narrowly as a type of action for which a nervous system is essential. It is true that nervous reactions are in the same evolutionary order as reactions in organisms not possessing a nervous system. This probably explains why so many writers have assimilated the two types of reaction as coming under the same name. For example, this is what Verworn has done. First he describes a reflex action in general. "The essence of tho reflex action consists in the fact that an element that appreciates stimuli and an element that reacts to stimuli are so put into relation with one another by a central bond, that every stimulus acting upon the appreciating element is conducted first to the center, and thence, as an impulse to a reaction, to the reacting element. Such a mechanism, in which every stimulus acting upon the sensory end calls out with

${ }^{1}$ L. T. Hobhouse, Mind in Evolution, London, rgor, p. 28.

2 Op. cit., p. $\mathbf{x}$.

i Op. cit., p. 2. 
machine-like certainty a reaction at the other end, is a reflex arc." 1 Then he describes it as found in a unicellular organism without a nervous system. "The most primitive form of a reflex arc exists in unicellular organisms, the cell body of which possesses both the sensory and the motor elements, and even functions also as the central bond for the two." 1 Finally he describes it as found in a multicellular animal with a nervous system. "What in all these cases is differentiated within a single cell, is in the nervous system of animals distributed to several cells. In the simplest case of the latter, three different cells are concerned. One cell, the sensory cell, receives the stimulus; from this a centripetal nerve path conducts to a central cell, the ganglion cell, and from here a centrifugal nerve path conducts to a cell that performs the reaction, the motor end cell. But this form of reflex arc is realized perhaps only in the invertebrates. In vertebrates, so far as the conditions are known, a fourth cell at least is interpolated in the arc, since in place of one ganglion cell at least two are present, one of which receives the stimulus from the sensory cell and conducts it to the other, while the other transfers the impulse to the motor end cell." 2

In similar fashion Jennings, after considering various meanings which have been given to the term reflex action, arrives at the very broad definition of a reflex "as an invariable reaction to a simple stimulus." ${ }^{3}$ This is to be expected of Jennings, for, as we have already seen earlier in this chapter, it is his tendency, like Loeb, to minimize the differences between the organisms without and those

1 M. Verworn, General Physiology, London, 1899, p. 576.

2 Op. cit., p. 577 .

Op. cit., p. 278. 


\section{The Evolution of the Nervous System 143}

with a nervous system. But, as I have contended, the differences between the behavior of an animal with a nervous system and the behavior of one without a nervous system are so great that it is worth while to emphasize them. Consequently $I \mathrm{am}$ in favor of restricting the term reflex action to the reactions of animals with a nervous system. For doing so we have the weighty authority of Sherrington. He defines reflex action first as follows: "These reactions, in which there follows on an initiating reaction an end-effect reached through the mediation of a conductor, itself incapable either of the end-effect or, under natural conditions, of the inception of the reaction, are "reflexes." 1 Then he restricts the meaning of the term as follows: "It seems better to reserve that expression for reactions employing specifically recognizable nerve processes and morphologically differentiated nerve cells." 2

I shall therefore use the term reflex action only in the restricted sense of meaning the reaction of muscle or gland or other effector organ caused by a nervous stimulus. Let us see what is the mechanism of this reaction. Parker says that it falls into three parts. "The first of these is the sense organ or receptor, which, as its name implies, receives the external stimulus; the receptor is also the seat of the production of the nerve impulse. The second is the central nervous organ or, as it may be called, the adjustor, which is concerned with directing the impulse toward the appropriate end organ and with modifying it in accordance with the peculiar reaction to be obtained. The third and last is

1 Charles S. Sherrington, The Integrative Action of the Nervous System, New York, I906, p. 6.

Op. cit., pp. 6-7. 
the effector or organ brought into action by the impulse, such as a muscle or gland." 1 Sherrington describes the reflex arc as including the following: "An effector organ, e.g., gland cells or muscle cells; a conducting nervous path or conductor leading to that organ; and an initiating organ or receptor whence the reaction starts." 2 It is to be noted that Parker includes a central nervous organ in the reflex mechanism, and Loeb implies the same in a passage which has already been cited. The use of the word reflex also seems to indicate that a central nervous organ must be included in the reflex mechanism, for it implies that the central nervous system is reflecting the stimulus received from the sense organ upon the motor organ just as a mirror reflects objects. But Sherrington does not include a central nervous organ, and it certainly seems unnecessary for a simple reflex, for which are needed only a sense organ and a motor organ with a conducting nervous path between them. It is to be noted, also, that a muscle or gland is included in the reflex mechanism. A muscle or gland is not a part of the nervous system, but it performs an essential part of a reflex action, - in fact, the most important part, - for it is the only part which is visible and which constitutes a part of the behavior of the animal. Therefore a muscle or gland must always be assumed as essential to a nervous reaction.

A simple reflex would take place if an effector organ responded to a receptor organ, while the rest of the organism remained quite indifferent to the reaction. But it is doubtful if a simple reflex ever takes place, for all parts of the nervous system are connected together so that it would hardly be possible for such a reaction to take place without

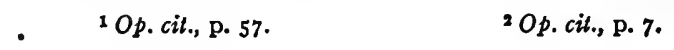




\section{The Evolution of the Nervous System 145}

affecting other parts of the system. ${ }^{1}$ If an organism possessed but one receptor and but one effector, a simple reflex might be possible, but it is doubtful if such an organism exists.

\section{Evolution of the Nervous System}

Let us now consider in somewhat more detail the evolution of the nervous system. As has been shown, the characteristic of conductibility possessed by nervous matter is not peculiar to it, for it characterizes, only in a lesser degree, protoplasm in general. Stimuli are carried through protoplasm either by mechanical or by chemical means. In a multicellular organism, if the intercellular material is solid, stimuli are likely to be carried by mechanical means; if the intercellular material is fluid, stimuli are likely to be carried by chemical means. But in both cases the conduction of the stimulus is delayed somewhat by the passage from one cell to another. Hence it was that where rapidity of conduction was needed, a special class of cells began to develop whose special function it was to carry these stimuli and thus connect the different parts of the organism together. These cells are usually elongated and stretch out protoplasmic threads in the direction in which the stimuli are to travel. Sherrington has summed up the evolution of the nerve cells in the following words: "But the internal interconnection of the multicellular organism is not restricted to intercellular material. Intercellular material is, after all, no living channel of communication, delicately responsive to living changes though it may be. An actual living internal bond

1 Cf. Sherrington, op. cit., pp. 7-8.

L1. 


\section{The Science of Human Behavior}

is developed. When the animal body reaches some degree of multicellular complexity, special cells assume the express office of connecting together other cells. Such cells, since their function is to stretch from one cell to another, are usually elongated; they form protoplasmic threads, and they interconnect by conducting nervous impulses. And we find this living bond the one employed where, as said above, speed and nicety of time adjustment are required, as in animal movements, and also where nicety of spatial adjustment is essential, as also in animal movements." 1

Just how these nerve cells evolved, it would be impossible to describe here in detail, but it goes without saying that in their case, as with all parts of the organism, variation and selection were the forces at work. The individuals which responded most readily and most advantageously to the stimuli of life-or-death importance would be selected out for survival, so that in course of time there evolved this system of cells which are peculiarly sensitive to stimuli. Thus the nerve cells are distinctly adaptive in their character. Loeb suggests this thought when he says that many reflex movements are "purposeful" in their character. ${ }^{2}$ He illustrates this statement by mentioning several reflexes which remove the organism from harmful stimuli. It is, however, unfortunate that he should use the term "purposeful," for this suggests that they are telic in their character, whereas he himself insists that there is no intelligence back of these reflexes necessarily, however well planned they may appear to be. The term "adaptive" certainly is much more suitable.

1 Op. cit., p. 5.

2 Op. cit., p. 2. 
But while we are discussing the evolution of the nervous system, we must not forget the effectors, which, as we have seen, form a necessary part of every reflex. There have been various theories as to the relation of the evolution of the effectors to the evolution of the nervous system. One theory has been that the nervous system evolved before the effectors. Another theory has been that they evolved at the same time. A third theory has been that the effectors evolved before the nervous system. In view of our foregoing statement that the effectors form a necessary part of every reflex, it does not seem likely that the nervous system would evolve before the effectors. We have already noted the adaptive character of the nerve cells. We have seen that they aid animals in the struggle for existence by making them more sensitive to stimuli which are of life-or-death significance. But it is evident that it would be of no assistance to these animals to be sensitive if they did not have effectors in the form of muscles which could take them away from harmful stimuli and propel them toward those that are beneficial. It therefore seems most probable that the evolution of the effectors began, to say the least, before that of the nervous system, though later the evolution of the two could go on together.

This is the position taken by Parker in his series of papers entitled "The Origin of the Nervous System and its Appropriation of Effectors." His arguments in behalf of this theory are very convincing. In the following words he shows how difficult it is to believe that receptors or sense organs could evolve before there were any effectors: "A receptor or sense organ alone would be of no service whatever to an animal; it would resemble a telephone 
receiver disconnected from the rest of the system. In a similar way the adjustor or central organ is useless without at least some other element in the reflex apparatus. The only mechanism sufficient in itself is the effector, which, if it can be brought into action by direct stimulation, may accomplish something serviceable to the animal. It is therefore improbable that we shall find multicellular animals that possess either receptors or adjustors without effectors, but it is conceivable that primitive metazoans may have effectors without other parts of the typical neuromuscular mechanism." 1 . He describes a certain species of sponge which possesses muscle cells in the form of sphincters, but no nervous mechanism, so that these effectors must be brought into action by direct stimulation. "Thus the sponge would represent the first stage in the differentiation of a neuromuscular mechanism, i.e., one in which the effector in the form of a primitive muscle-cell is the only element present. In my opinion it is around these contractile cells that the nervous organs of the higher metazoans have developed, and I therefore believe that these effector elements are the most primitive members in the typical neuromuscular mechanism." 2 Such independent effectors may be found also in higher animals, as Parker indicates in the following passage: "If independent effectors occur in sponges, it is not unlikely that they may be present in the higher animals, and as possible examples of these the sphincter pupillæ of the eye in vertebrates and the heart-muscle may be considered." 3 These are the facts that lead Parker to the conclusion that "effectors in the form of muscles preceded in an evolutionary sense the receptors and ad-

$$
{ }^{1} O \text { p. cit., p. } 58 . \quad 2 O p \text {. cit., pp. } 59-60 . \quad 2 O p \text {. cit., p. } 60 .
$$


justors, and formed the centers around which these organs developed later." 1

Following the independent effector stage, according to Parker, comes the receptor-effector stage, and then the central nervous organs develop and take their places between the receptors and effectors. These three stages he describes as follows: "The first was that of the independent effector, the muscle which was brought into action by the direct influence of environmental changes as seen in the pore sphincters of sponges. The second stage was that of the combined receptor and effector in which the receptors, in the form of diffuse sensory epithelia or specialized sense organs, served as delicate triggers to set the muscles in action and thereby render the effectors responsive to a wider range of stimuli than they would be under independent stimulation. Finally, the third stage is seen in the complete neuromuscular mechanism in which a central nervous organ or adjustor has developed between the receptors and the effectors. This adjustor serves as a switchboard for nervous transmission and a repository for the effects of nervous activities." 2

In his last paper, Parker discusses what he calls "the appropriation of effectors." He calls attention to the fact that in the third stage described above the nervous system is connected not only with muscles, but also with electric organs, chromatophores, glands, luminous organs, etc. These effectors, he thinks, are recent acquisitions by the nervous system. He thinks also that independently and newly developed muscles have been acquired by the nervous system. I have not the space to discuss fully his argu-

$$
{ }^{1} \text { Op. cit., p. } 64 \text {. }
$$$$
2 \text { Op. cit., p. } 338 .
$$ 
ments and the many illustrations he gives. But it appears from his discussion of the subject that the nervous system may at any time acquire control over more effectors, thus extending its sway over a larger part of the animal organism. He sums up this process in the following words: "We must picture to ourselves as steps in the evolution of the nervous system, not only the independent origin of muscle around which the nervous organs subsequently develop, but also the independent origin of other effectors such as chromatophores, glands, and phosphorescent organs and the secondary appropriation of many of these by a developing nervous system. This principle of appropriation I believe to be as significant in elucidating the present condition of the nervous system and its appendages, as the principle of evolutionary sequence of parts, muscle, sense organ, and central nervous organ." 1

\section{Description of the Nervous System}

Let us now consider in greater detail the principal characteristics of a fully developed nervous system as it is to be found in any one of the higher animals. We have noted that the receptor cell is more sensitive to external stimuli than other peripheral cells. This statement is, however, not quite accurate, for while a receptor is very sensitive to certain kinds of stimuli, it may be less sensitive than the ordinary cell to other stimuli. Thus a receptor is, as Sherrington puts it, "a mechanism more or less attuned to respond specially to a certain one or ones of the agencies that act as stimuli to the body." ${ }_{2}$ This selective excitability of the receptors or sensory organs has undoubt-

$$
1 \text { Op. cit., p. } 345 .
$$$$
2 \text { Op. cit., p. } 12 .
$$ 


\section{The Evolution of the Nervous System 151}

edly arisen through adaptation and is of great utility to the organism. Because of the high degree of specialization which has developed among these receptors, the organism can adjust itself with much more nicety to its surroundings. The functional value of these receptors is therefore very great. Sherrington has summed up the function of the receptor in the following words: "The main function of the receptor is therefore to lower the threshold of excitability of the arc for one kind of stimulus, and to heighten for all others." 1

This specialization on the part of the receptors is sometimes described under the name of "the law of the specific energy of the sense organs." Loeb criticizes this law as follows: "This so-called law of the specific energy of the sense organs is not peculiar to the sense organs; it applies, as was emphasized by Sachs, to all living matter; it even holds good for machines. It is in reality only another expression for the fact that the eye, the ear, and every living organ are able to convert energy in but one definite form - that is, that they are special machines." 2 This is all true enough, for it goes without saying that every particle of matter will transmit energy somewhat differently from every other particle of matter, for there are no two particles of matter which are absolutely alike. But the difference between the way in which each sense organ transmits energy is so great from that of other kinds of protoplasm, and the functional value of these differences is of such importance, that it is, I believe, justifiable to emphasize these differences by speaking of the specific energies of the sense organs. After a stimulus has been received by the receptor, it

I Op. cit., p. I2.

2 Op. cit., pp. 290-29r. 
must be conducted to the effector if it is to affect the behavior of the animal. Sherrington states that nerve cells have to an unusual degree the power to conduct stimuli. "They have in exceptional measure the power to spatially transmit (conduct) states of excitement (nerve impulses) generated within them." 1 But this power arises not only out of the composition of the nerve cells, but also out of their shape. As has already been indicated, many of the nerve cells are elongated and stretch out long protoplasmic threads in the direction in which the stimulus is to travel. In some cases the stimulus may go all the way to the effector by one nerve cell, though probably no such case exists in the higher animals. In such cases the conduction would be most rapid and with the smallest loss of force, for it is the passage from one cell to another which delays a stimulus and weakens it. But even if it has to pass through more than one nerve cell, the number will be much fewer than if they were ordinary cells, so that the conduction will be much more rapid and with smaller loss of force than if the conduction were by means of ordinary cells.

The receptors or sense organs vary greatly amongst themselves. As we have already seen, in each case there is represented a characteristic of all protoplasmic matter which has become highly specialized in the cells of the sense organ. For example, the essential part of the eye consists of cells which have become highly sensitive to photic stimuli. As we have seen in a preceding chapter, a rudimentary eye is to be found even in the lowest organisms, such as some of the protozoa, in the form of a spot which is unusually sensitive to light. The sense organs 


\section{The Evolution of the Nervous System 153}

of taste and of smell consist of cells which are highly sensitive to chemical stimuli. The sense organs of touch and of hearing consist of cells which are highly sensitive to mechanical stimuli. Some of these sense organs have become highly complex mechanisms, such as the eye with its lens, cornea, retina, etc., and the ear with its otoliths, cochlea, circular canals, etc. I have not the space to describe in detail how these organs have evolved, some suggestion of which has been given in preceding chapters, but must discuss how these organs are connected with the nervous system.

It will be noted that the sense organs are not necessarily nerve cells, just as we have already noted that the effectors are not nerve cells. As a matter of fact, most of the sense organs are not nerve cells. It will also be noted that the sense organs are necessarily peripheral; that is to say, they are on a surface which is exposed to stimuli. This does not mean that they are necessarily on the outside of the body. Many of them are on the surface of the intestines and the other internal organs. But all of the sense organs are connected with effectors, in the higher animals at any rate, by means of nerves.

Wundt states that a nerve ends in a peripheral organ in one of two ways. "The termination of a nerve in the peripheral organs may take one of two forms. Either the ends of the neuritic threads divide up into a fascicle or network of finest dendritic fibrils, that terminate freely along the elements of other, non-nervous tissues; or the neuritic thread passes directly over into a terminal cell situated within or between the organs. The terminal cell may be an original nerve cell, pushed out towards the periphery of the body; or it may have acquired this character later on in the course of development, by the penetration of a nerve fibril into an epithelial cell. The two forms of nerve termination occur side by side, in these their characteristic 


\section{The Science of Human Behavior}

differences, in the different sense-organs, where they are evidently connected with essential differences in the mode of sensory excitation." 1

The first type of nerve termination is illustrated in the termination of sensory nerves in the skin where the neurite when it enters the epithelium breaks up into a reticulum of delicate fibrils. It is also illustrated in the nerve endings in muscles. The second type of nerve termination is illustrated in the organ of smell, in which every nerve fiber enters a nerve cell in the olfactory mucous membrane.

\section{The Neurone}

In order to understand more clearly how a stimulus is carried from receptor to effector, we must examine in more detail the nerve cell. As has already been indicated, a nerve cell is usually somewhat elongated and sometimes sends out fibers which attain great length. It has sometimes been thought that these fibers are not a part of the nerve cell. But it is easy to show that they are a vital, trophic, as well as a functional part, of the nerve cell, because if they are cut off from the central part of the cell they perish. Because of this failure on the part of some to recognize that these fibers form a part of the nerve cell, it has become somewhat customary to call the nerve cell, including all of its appendages, a neurone. ${ }^{2}$

The neurones vary greatly in shapes and sizes, and so do their processes. In many neurones one of the processes is much longer than the rest and is called the neurite or axone or axis-cylinder or neuraxone or nerve process. Some of these neurites are very long as, for example, those which run from the spinal cord to the ends of the toes. Some neurites give off at intervals branches called collaterals or paraxones, which connect them with other neurones. The neurone has also

1 W. Wundt, Principles of Physiological Psychology, London, I904, Vol. I, p. 47.

2 This term was coined by Waldeyer in $189 \mathrm{r}$. See his paper in the Deut. Med. Wochenschrift, I891, p. 50. 


\section{The Evolution of the Nervous System 155}

certain protoplasmic processes which are called dendrites or dendrons or cytodendrites. These dendrites are usually shorter than the neurites and are much branched. They serve like the collaterals to connect the neurones with each other and also to receive stimuli from receptors and conduct them to the neurones. It has been thought by some that the sole function of the neurites is to conduct stimuli from the neurones to the effectors, but it appears now that they have other functions to perform as well.

The exact mode of connection between the neurones is a matter of importance. As has been indicated, the connection is through the dendrites and collaterals. But inasmuch as each neurone forms a separate cell, it is thought by many that there must be a surface of separation between the nerve cells just as there is between other types of cells. This is the synaptic theory of the connection between nerve cells. $^{1}$ In this theory the junction between the neurones is called the synapse, and it is believed that the cells are separated by a thin transverse membrane. It is, however,

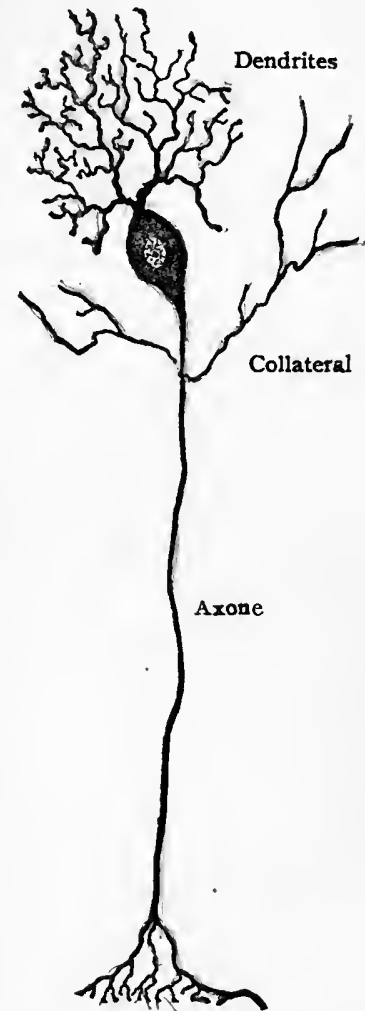

Telodendrion

Frg. 8. - Diagram of a typical neurone. (After Piersol.) difficult to prove this theory because of the microscopic character of this membrane. Furthermore, it demands

1 By some the synaptic theory is included as an essential part of the so-called "neurone theory" or "neurone doctrine." (See W. H. Howell, Textbook of Physiology, Philadelphia, rgo6, pp. $\mathrm{r} 2 \mathrm{r}-\mathrm{r} 23$. ) But it is questionable if the neurone necessarily involves the synapse. 


\section{The Science of Human Behavior}

an explanation of how stimuli pass easily and rapidly through this membrane. Another theory of the connection between the neurones is the neurofibrillar or intercellular bridge theory according to which the neurones are connected by protoplasmic threads which pass directly from one cell to the other. This theory also is a difficult one to prove, but both of these theories have had considerable influence upon neurology.

\section{The Central Nervous System}

Most of the neurones are located in what is called the central nervous system. Just how this system evolved is hard to explain, and I have not the space to attempt to do so here. According to some writers the nerves were at first external and then withdrew into the interior of the body to form the central nervous system. ${ }^{1}$ According to some of the supporters of the synaptic theory, the central nervous system has come into being through the synapse. "The synaptic nervous system has developed as its distinctive feature a central organ, a so-called central nervous system; it is through this that it brings into rapport one with another widely distant organs of the body, including the various portions of the diffuse nervous system itself." ${ }_{2}$

But in whatever way the central nervous system may have evolved, we have to accept it to-day as an important part of the nervous system in the higher animals. Before ending this chapter I wish to describe briefly the central nervous system in vertebrates.

The vertebrate central nervous system consists of the

1 Cf. H. H. Wilder, History of the Buman Body, New York, 1909, p. 465.

2 Sherrington, op. cit., p. 3 I 2. 
spinal cord, which is divided up into segments which correspond with the segments of the spinal column, and the brain, which is made up morphologically of a number of segments of the spinal cord which have become highly differentiated. It is evident that the vertebrates have a great advantage over the invertebrates in developing the central nervous system, because of the serial arrangement of the parts of the organism. The results of this advantage are shown in two ways. ${ }^{1}$ In the first place, the central nervous organs of the vertebrates display a much greater number of long neurones over short ones. In the second place, the vertebrates possess a very much larger number of association neurones. It is also to be noted that in the later stages of the evolution of animals the central nervous system has evolved very rapidly and has thus furnished a basis for mental evolution, while the sense organs have improved much less. ${ }^{2}$ The sense organs reached a high state of efficiency earlier in the evolution of animals and have not improved very much since. The eye in some kinds of fish is almost as good as that of the mammal, while the sense of hearing is very acute in some of the reptiles and amphibians.

${ }^{1}$ Cf. G. H. Parker, op. cit., p. $262 . \quad 2$ Cf. H. H. Wilder, op. cit., pp. $465-466$. 


\section{CHAPTER IX}

THE FUNCTIONS OF THE NERVOUS SYSTEM

The kinds of reflex arcs, I59. - Facilitation, I60. - Inhibition, I60. - The common path, r6o. - The integrative function of the nervous system, I6r. - Mechanical, chemical, and nervous integration, r6r. - The functional divisions of the nervous system, I62. - The origin of the head and brain, 163. - The functions of the distance receptors, I64. - Extero-ceptive, proprio-ceptive, and intero-ceptive receptors, $165 .-$ The neuromeres, $167 .-$ The divisions of the brain, $167 .-$ The functions of the cerebellum, I68. - The functions of the cerebrum, 172. - The divisions of the cerebrum, $\mathbf{1 7 4}$.

Ir is evident from the brief description of the evolution of the nervous system in the preceding chapter that we still have much to learn about this evolution. But what we do know is very suggestive with respect to the functions of the nervous system, so that the preceding chapter has incidentally thrown some light upon these functions.

We still have much to learn, also, about the functions of the nervous system. But the subject is one of supreme importance in the study of behavior, especially with respect to the higher animals, because most of the actions of these animals are determined directly by the nervous system. Most if not all of the data presented in this chapter are , certain, but some of the theories discussed are very uncertain and may be disproved. I shall begin the discussion of this subject by describing the different kinds of reflexes in the vertebrates. 
The Functions of the Nervous System 159

\section{The Reflex ArC}

There are few if any reflexes in the vertebrates which do not pass through the central nervous system. Three levels of reflex arcs are sometimes distinguished. ${ }^{1}$ The first is an arc on the spinal level. In this arc the stimulus passes by means of the processes of a neurone from the receptor organ to the cell body of a sensory neurone in the central nervous system. From this neurone it is transferred to a neurone connected with the effector organ and by means of whose processes it is carried to the effector. The second level of arcs is where loops are formed on the arcs of the spinal level by their neurones becoming connected with each other so that these reflexes influence each other. The third level is reached when the brain de-

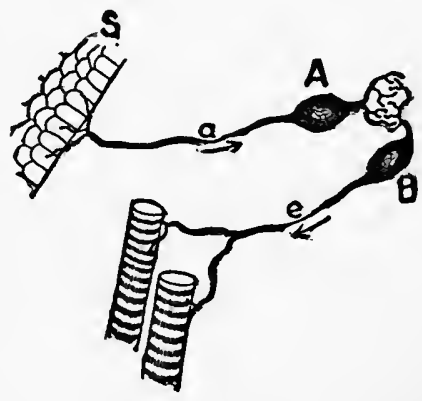

FIG. 9. -Diagram illustrating a reflex arc and showing fundamental units of nervous system. $A$, sensory neurone, conducting afferent impulses by its process $(a)$ from periphery $(S) ; B$, motor neurone sending efferent impulses by its process $(e)$ to muscle. (After Piersol.)

velops so that the reflexes become interconnected in a much more complex fashion. The cerebrum, which is the most important part of the brain, is made up largely of association centers; that is to say, of neurones which are not directly connected with receptor or effector organs, but whose processes are devoted entirely to connecting other neurones with each other.

As a result of these connections established between

1 Cf. W. McDougall, Physiological Psychology, London, I905, pp. 20-2I. 
neurones by means of the central nervous system, two very important phenomena take place. ${ }^{1}$ The first is facilitation; that is to say, the reënforcement of the excitement of a motor system by the stimulation of other sensory neurones connected with the same motor system. The second is inhibition, or the partial or complete prevention of the spread of excitement from a sensory neurone to a motor system, caused apparently by the simultaneous excitement of some other motor system. A sensory neurone can receive stimuli only from the sense organ with which it is connected. But an effector may receive stimuli from several sensory neurones. These facts have been stated in what Sherrington calls the principle of the common path, which is that "while the receptive neurone forms a private path exclusively serving impulses of one source only, the final or efferent neurone is, so to say, a public path, common to impulses arising at any of many sources of reception." 2 This common path may be used by like reflexes at the same time, but not by unlike reflexes, for an effector cannot be made to do two things at the same time. "Unlike reflexes have successive but not simultaneous use of the common path; like reflexes mutually reënforce each other on their common path." 3 These characteristics of the common path make it a coordinating mechanism which prevents confusion by restricting the use of the effector to one action at a time. "The resultant singleness of action from moment to moment is a keystone in the construction of the individual whose unity it is the specific office of the nervous system to perfect. The interference of unlike reflexes and the alliance of like

1 Cf. Wundt, op. cit., pp. 70-77; McDougall, op. cit., pp. 35-36; Sherrington, op. cit., p. 36.

Op. cil., p. 116.

Op. cit., p. 233 . 


\section{The Functions of the Nervous System 161}

reflexes in their action upon their common paths seem to lie at the very root of the great psychical process of 'attention." " 1

\section{INTEGRATION}

The coördination effected by the common path brings our attention forcibly to the main function of the nervous system in general; namely, its integrative function. I have already referred to this function, and it has been illustrated over and over again in the preceding description of the nervous system. Indeed, in a sense the integrative function includes all the other functions of the nervous system. The best exposition of this subject has been made by Sherrington in his able work entitled The Integrative Action of the Nervous System, which has already been cited a number of times, and I shall follow his treatment in the main in this discussion.

There are several forms of integration in the animal organism. There is first the mechanical combination of the cells of the body into a single mass by means of muscles, connective tissue, etc. In the second place integration is effected by means of certain chemical agencies which establish communication between different parts of the organism. We find these agencies in the reproductive organs, in the digestive organs, and in the circulation of the blood. Both of these forms of integration are of great importance and indirectly affect very greatly the behavior of the animal. But the form of integration which is of most interest to us is the integrative action of the nervous system, because this determines directly and to a large extent the behavior of the animal. 
In its integrative action the nervous system "works through living lines of stationary cells along which it dispatches waves of physico-chemical disturbance, and these act as releasing forces in distant organs where they finally impinge." 1 The result is that nervous integration is usually more rapid than the other forms of integration in which the means of communication is intercellular material, such as connective tissue, or where material in mass has to be transferred, as in the circulation of blood. The unit mechanism in nervous integration, as in the nervous system as a whole, is the reflex arc, "because every reflex is an integrative reaction, and no nervous action short of a reflex is a complete act of integration." 2 Nervous integration is therefore accomplished entirely by means of reflexes, of which two kinds may be distinguished, the simple reflex, of which there are none, probably, at any rate in the higher animals, and the compound reflex. All or most of the behavior of the higher animals is therefore reflex, because all or most of it is integrated by the nervous system. There are differences of opinion as to this which will be discussed in the chapter on consciousness.

\section{Functional Divisions of the Nervous System}

The functional divisions of the nervous system are the somatic sensory, the somatic motor, the visceral sensory, and the visceral motor. ${ }^{3}$ The visceral nerves, sometimes called the sympathetic system, are not under voluntary control, and it was formerly believed that they were not connected with the other parts of the nervous system which

1 Op. cit., pp. 3-4.

op. cit., p. 7.

${ }^{3}$ Cf. J. B. Johnston, The Neroous System of Vertebrates, Philadelphia, 1906, pp. IOI-IO3. 


\section{The Functions of the Nervous System 163}

are under voluntary control, but it is now known that such connections exist. ${ }^{1}$ As has already been stated, the central nervous system in vertebrates is divided into segments called neuromeres, which correspond with the segments or metameres of the spinal column. In other types of animal organisms, also, we find a segmental arrangement, as, for example, in many radiates. But a higher development has been possible in the species in which the segments are arranged serially. In a radiate each segment bears the same relation to the common axis and to the other segments, so that there is not much chance for differentiation. For example, the mouth is at the same distance from all the segments, and in other ways the conditions of life for each segment are about the same as for all. But in a serially segmented animal there is much opportunity for differentiation among the segments. ${ }^{2}$ For example, the segments from which the limbs begin are sure to develop a more complex nervous mechanism. The segments are no longer equidistant from the mouth. Most important of all, certain segments now go first in locomotion, and consequently develop far beyond any other segment. This marks the beginning of the head and the brain, which I shall now discuss.

It is easy to see how the leading segments would develop much farther than the other segments. In the first place, more impressions fall upon these segments, thus developing them more than the other segments in the individual. Unless, however, these effects upon these segments in the individual were transmitted by inheritance, they would have no effect upon the species. In all probability they are not

1 Cf. McDougall, op. cit., p. I6.

${ }^{2}$ Cf. Sherrington, op. cit., p. 315. 
transmitted, but there are other forces at work to cause greater development of these segments in the species. These segments are the first ones usually to meet the forces of the environment which are beneficial or harmful to the animal. Consequently, these segments are subjected to a very rigorous process of selection, and only those individuals tend to survive whose receptors in the leading segments adapt them to their environment. This results in the refinement of these receptors until they are highly adaptive.

\section{Distance Receptors}

Especially significant in this process is the evolution of what Sherrington calls the "distance-receptors," by which he means "receptors which react to objects at a distance." 1 In one sense no receptor could be a distance-receptor, because every receptor reacts directly to mechanical forces which act upon it. Thus receptors which are reacting to photic stimuli react directly to vibrations in the ether; receptors which react to auditory stimuli react directly to vibrations in air or water or whatever the conducting medium is. In this respect they are similar, for example, to tango-receptors, which react directly to massive, material objects which come into contact with them. But they are distance-receptors in the sense that they determine reactions which adapt the individual to objects which are at a distance, but from which are emanating forces which directly affect the receptors.

So it is that a number of these distance-receptors begin to develop on these leading segments. "The retina is thus Op. cit., p. 324. 
a group of glorified 'warm spots,' the cochlea a group of glorified 'touch spots.' Again, a group belonging to the system adapted to chemical stimuli reach in one of the leading segments such a pitch of delicacy that particles in quantity unweighable by the chemist, emanating from substances called odorous, excite reaction from them." 1 The organs of vision, hearing, and smell are the principal ones of the distance-receptors. In the case of smell, as indicated above, the sense organ is acted upon by minute particles so that it may appear that it is not a distance-receptor. But these objects acting upon the organ cause the individual to adapt itself to the object at a distance from which these particles are emanating, so that this sense organ may be called a distance-receptor.

Just how the animal organism has acquired the ability to adapt itself to these objects at a distance, it is difficult to explain. Sherrington says that these receptors "initiate sensations having the psychical quality termed projicience." 2 That is to say, these sensations are projected into the world outside the individual, and this projicience "refers them, without elaboration by any reasoned mental process, to directions and distances in the environment fairly accurately corresponding with the 'real' directions and distances of their actual sources." This property of projicience belongs only to sensations initiated by what Sherrington calls the "extero-ceptive" receptors. By extero-ceptive he means those which lie on the surface of the body, and distinguishes them from the proprio-ceptive receptors, which lie buried beneath the surface, and the intero-ceptive receptors, which

1 Op. cit., p. 324 .

${ }^{2} O$ p. cit., p. 324 . Here, as elsewhere in his writings, Sherrington uses psychological terms in a rather loose fashion. 
line the surfaces of the internal organs. It is evident that the distance-receptors would have to be extero-ceptive, because the proprio-ceptive and intero-ceptive receptors hidden beneath the surface could not very well be affected by the forces emanating from the objects at a distance to which the individual reacts.

That these distance-receptors have great survival value, must be quite evident. With the aid of them an animal becomes aware of, and is thus able to avoid, the objects which are injurious to it and to bring itself into contact with those which are beneficial to it. Thus the head, which is constituted of these leading segments in which most of the distance-receptors are located, is of the greatest importance to the animal, because it "moves habitually into that part of environmental space which has been already explored by the distance-receptors of its own leading segments," and inasmuch as the head "has the mouth, it takes in the food, including water and air, it has the main receptive organs providing data for the rapid and accurate adjustment of the animal to time and space. To it the trunk, an elongated motor organ with a share of the digestive surface, and the skin, is appended as an apparatus for locomotion and nutrition. The latter must of necessity lie at the command of the great receptor-organs of the head." 1

\section{The Brain}

But so far have been discussed only the receptor organs of the head, and most if not all of these are not nerves, so that there still remains to be discussed the nervous equip- 


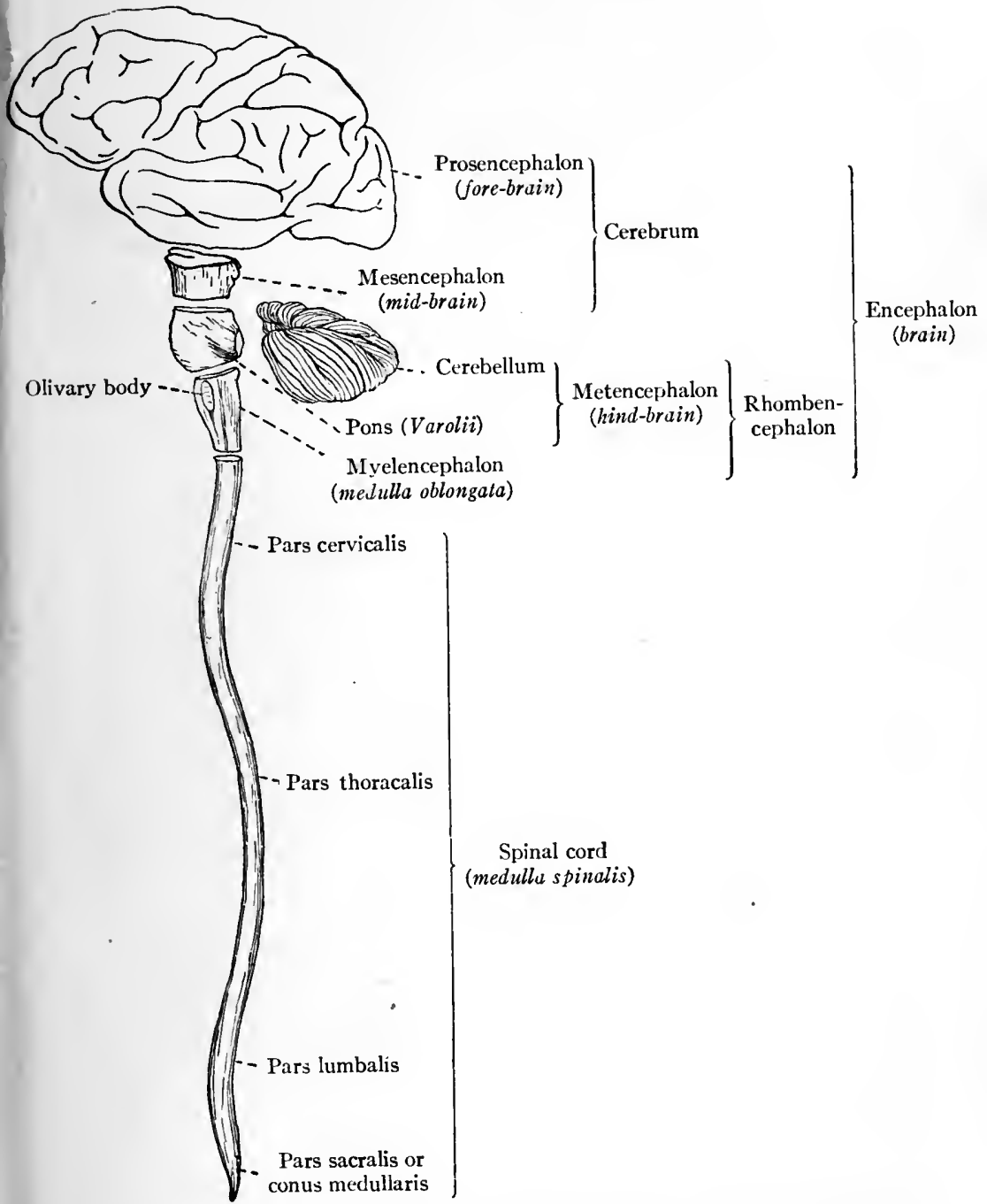

FIG. 10. - Diagram illustrating the divisions of the central nervous system. (After Morris.) 



\section{The Functions of the Nervous System 167}

ment of the head. This equipment consists of the neuromeres or sections of the spinal cord which correspond to the segments of the spinal column from which evolve the skull or bony inclosure of the head. These neuromeres have developed so greatly as to be very large and complex as compared to their original state. This development is due to the same causes as the enlargement of the sections of the spinal cord where the fibers from the limbs come in. The neuromeres of the leading segments receive stimuli from many more receptors than do most of the neuromeres of the spinal cord. Furthermore, many of these are distancereceptors from which are received sensations for which many association paths are established.

The neuromeres of the head constitute the brain. The brain consists of four parts - the cerebrum, the cerebellum, the pons Varolii, and the medulla oblongata. The medulla oblongata connects the spinal cord with the pons, and the pons connects the other three parts of the brain with each other. The most important parts of the brain are the cerebrum and the cerebellum, and I shall now discuss their functions briefly.

It is reasonable to suppose that in a part of the body which is so highly differentiated as is the brain, there must be a considerable differentiation of function. This would be in accordance with what we see of the physiological division of labor in other parts of the organism. But while there has been a great deal of investigation of this subject, there is still great uncertainty as to the exact functions of these parts of the brain. There are difficulties involved in every method of research used in studying this problem. The anatomical method of studying the anatomy of the 
brain furnishes certain presumptions as to how the functions are distributed among the parts of the brain. But the information thus gained is not certain, because such anatomical study cannot be carried on to a very great extent in the living subject, so that most of these researches are made when the brain is not functioning. The method of extirpation is a valuable one, but is not always a certain one, because the surgical effect of the shock of the operation may be such as to cause changes greater than the mere effect of the extirpation. Furthermore, other parts of the brain sometimes compensate for the parts which have been extirpated, so that it is impossible to tell exactly what are the functions of the extirpated parts. Many more researches will have to be made before the exact functions of all parts of the brain can be known. I shall therefore limit myself to citing the opinions of a number of authorities as to the functions of the cerebellum and the cerebrum.

\section{The Cerebellum}

Herbert Spencer in his famous treatise on psychology suggested that "the cerebellum is an organ of doubly-compound coördination in space; while the cerebrum is an organ of doubly-compound coördination in time." $1 \mathrm{He}$ believed that this theory was supported by the facts that "the cerebellum is unusually developed in birds of prey, which have to coördinate with great accuracy the relations of distance, direction, and complex form, as well as very precisely to coördinate the involved movements appropriate to these involved impressions. And there is, on the other hand, the fact that the cerebrum predominates in ${ }^{1}$ Principles of Psychology, 3d edit., New York, 1895, Vol. I, p. 6r. 


\section{The Functions of the Nervous System 169}

creatures showing, like ourselves, the power of adapting, throughout long periods, concatenated compound actions to concatenated compound impressions." 1 This is a rather plausible theory because of the relative positions of these two parts of the brain. The cerebellum is behind and somewhat below the cerebrum and is an enlargement of certain segments of the spinal cord back of the segments from which the cerebrum has developed, these being the first segments of the spinal cord. The cerebellum therefore receives stimuli from the spinal cord before they reach the cerebrum, and is able to make simpler reactions, such as may involve coördinations with respect to space. The cerebrum is reached later and makes the more complex coördinations of sequence, such as are involved with respect to time. But this is too simple a theory by which to explain the differentiation of functions in the brain, and I shall now pass on to some of the later theories.

There has been much difference of opinion as to the functions of the cerebellum. Some have overestimated and others have underestimated its importance, and there is much uncertainty both as to the importance and the nature of its functions. But there appears to be a rather widespread opinion that it has a direct control over the movements initiated in the cerebrum, and serves to coördinate these movements. Thus Wundt speaks as follows: "The cerebellum appears to be intended for the direct regulation of voluntary movements by sense impressions. If this hypothesis be correct, it will, accordingly, be the central organ in which the bodily movements incited from the cerebrum are brought into harmony with the position of the 1 Op. cit., p. 62. 
animal body in space. This conception agrees sufficiently well with our anatomical knowledge of the course of the lines of conduction, incoming and outgoing." 1

Johnston expresses a similar opinion, giving concrete examples of the effect of its extirpation in animals. "What then is the function of the cerebellar cortex? Disease of the cerebellum in man and its extirpation in animals always results in disturbances of voluntary muscular action. Animals from which one hemisphere or the whole cerebellum has been removed are unable to stand or walk until they have learned to make compensatory efforts. Does the cerebellum have the special function of maintaining the equilibrium, or is it necessary for the coördination of muscular contractions with reference to definite movements? In the results of experimental investigations on mammals the function of the cerebellum which stands out most prominently is different from either of these. Dogs which have lost one cerebellar hemisphere, although they are unable to stand or walk, can swim well in water (which supports their body weight), both coördinating their movements and maintaining their equilibrium. Such animals learn after a time to compensate for the loss of the cerebellum by certain voluntary modifications of their movements; e.g., curving the spine so as to bring the center of gravity over the sound legs, spreading the feet wide apart, etc. They can then stand and walk. These and other facts show that the loss of the cerebellum does not involve loss of the power of equilibration nor of cutaneous or muscle sense, on which the power of coördinated movements depends, but does result in weakness of muscular action on the injured side. It seems, therefore, that the cerebro-spinal mechanisms are sufficient to carry out all voluntary movements without the aid of the cerebellum, but the movements are lacking in strength, precision, and regularity. The cerebellum is not shown to be a necessary link in the nervous mechanisms which control muscular action, but it seems to add something to the voluntary movement. According to Luciani the function of the cerebellum is to maintain the tone of muscles during rest, to increase the energy of contraction when called forth by voluntary impulses, and to determine the rhythm of motor impulses. In this way the imperfect actions of the dog deprived of 


\section{The Functions of the Nervous System 171}

its cerebellum would be perfected into normal movements. Whether the cerebellum cortex actually serves other functions, such as the coördination of specific movements, remains for further investigation to decide." 1

Sherrington suggests that the cerebellum is the head ganglion of the proprio-ceptive system. It has already been indicated that the term proprio-ceptive as used by him refers to the receptors buried beneath the surface. $\mathrm{He}$ says that the central nervous organ of any system of nerves is likely to be located at the point where the largest number of nerves from that system enters the central nervous system. It appears that this is true for the cerebellum with respect to the proprio-ceptive system, for in the cerebellum "converge internuncial paths stretching to this mechanism from the central endings of various proprio-ceptive neurones situate in all the segments of the body. There afferent contributions from the receptors of joints, muscles, ligaments, tendons, viscera, etc., combine with those from the muscular organs of the head and with those of the labyrinthine receptors themselves. A central nervous organ of high complexity results. Its size from animal species to animal species strikingly accords with the range and complexity of the habitual taxis of the skeletal musculature. This central organ is the cerebellum." 2 Sherrington believes that this theory harmonizes with Spencer's theory, which has been cited; namely, that the cerebellum is the organ for coördination in space, and with the later theory which has been illustrated from Wundt and Johnston; namely, that it is the organ for the coördination of voluntary movements. Inasmuch as many of the proprio-ceptive

Op. cit., pp. 249-250.

2 Op. cit., p. 348. 


\section{The Science of Human Behavior}

receptors are located in the muscles, tendons, joints, etc., which directly determine the movements of the body, it seems plausible that the central nervous organ of the proprio-ceptive system should have a large measure of control over the movements of the body.

\section{The Cerebrum}

The cerebrum is the largest and the most important nervous organ in the highest animals. All writers on the brain agree in attributing great importance to the cerebrum, though their theories as to why it is so important vary somewhat. Wundt says, after describing the effects upon the individual of the extirpation of the cerebrum, that "these final abrogation phenomena lead us to the general, and, we must also admit, indefinite conclusion that the intelligence, the higher affective processes, and the compound voluntary actions are conditioned upon the integrity of the cerebral hemispheres." 1 He states further that this conclusion drawn from the phenomena of abrogation is confirmed by results from comparative anatomy, evolutionary biology, and anthropology. "Comparative anatomy shows that the mass of the cerebral lobes, and more especially their superficial ridging by fissures and gyres, increase with increasing intelligence of the animal. This law is, however, limited by the condition that both factors, mass and superficial folding, depend primarily upon the size of the body." 2 This is a well-known fact for which much evidence has been gathered. For example, the average brain weight of a man's brain in European races is 1360 grams, and that of a woman's brain of the same races is I2I I grams, while

$$
1 \text { Op. cit., p. } 284 \text {. } 2 \text { Op. cit., p. } 286 .
$$


the average brain weight of the great anthropoid apes (gorilla, chimpanzee, and orang-utan) is only 360 grams. $^{1}$ Thus in the orang-utan the brain represents only one half of one per cent of the body weight, while in European man the proportion is at least three per cent.

Sherrington states an interesting theory to the effect that the cerebrum is the ganglion of the distance-receptors. We have already seen that he considers the cerebellum the head ganglion of the proprio-ceptive system. The cerebrum would then be the ganglion of those extero-receptors which receive stimuli from a distance. He states that the reflexes which start from the distance-receptors are connected with the cerebrum, and that if the cerebrum is extirpated the reactions which are stimulated by these receptors are destroyed in large part. "The distance-receptors are the great inaugurators of reaction. The reduced initiation of action which ensues on ablation of the cerebrum seems explicable by that reason. The curtailment which ensues is indicative of damage which their removal inflicts on reactions generated by the distance-receptor organs. By a high spinal transection the splendid motor machinery of the vertebrate is practically as a whole and at one stroke severed from all the universe except its own microcosm and an environmental film some millimeters thick immediately next its body. The deeper depression of reaction into which the higher animal as contrasted with the lower sinks when made spinal signifies that in the higher types more than in the lower the great distance-receptors actuate the motor organ and impel the actions of the individual. The deeper depression shows that as the individual ascends the scale

${ }^{1} \mathrm{~J}$. Deniker, The Races of Man, London, 1900, pp. 17-18. 
of being, the more reactive does it become as an individual to the circumambient universe outside itself." 1

The cerebrum is divided into hemispheres, and these hemispheres may be divided into three parts. The first part connects the cerebrum with the rest of the brain and is a thick bi-lobed mass consisting of groups of nerve cells through which pass many large bundles of fibers. This part is called the basal ganglion or corpus striatum. "Lying in front of and below the corpus striatum and forming part of the lower and mesial wall of the hemispheres at the anterior end are the olfactory bulb and olfactory lobe. On the ventral surface of the striatum is the nucleus amygdala, which is continuous caudally with the pyriform lobe and the hippocampus in the temporal region of the hemisphere. These several structures, together with the fornix and hippocampal commissure, constitute the second main portion of the hemisphere, and may be spoken of collectively as the central olfactory apparatus. All the rest - much the greater part - of the cerebrum is concerned with sensory impulses from the external world which come from various parts of the body, including the special sense organs of sight and hearing; with the correlation of these impulses with one another and with habitual tendencies produced by previous actions; with voluntary impulses sent out to arouse, direct, or inhibit actions in response to stimuli; with sensations; and with thought processes. This portion of the cerebral hemispheres may be spoken of as the somatic pallium." "

We are most interested in the pallium or cerebral cortex in which the highest functions of the brain are performed. The study of this part of the cerebrum is in the main the study of cerebral localization; that is to say, the study of how these functions are localized in the brain. I shall take up this subject in the next chapter.

$$
{ }^{1} \text { Op. cit., pp. 351-352. } 2 \text { Johnston, op. cit., p. } 293 .
$$




\section{CHAPTER $\mathrm{X}$}

\section{CEREBRAL LOCALIZATION}

Phrenology, 175 - Anatomical study of cerebral functions, I76. - The anatomy of the brain, 177. - White and gray matter, 178. - The cerebral cortex, 178. - Theories of cerebral localization, 179. - The association areas, 18r. - The cerebral fibers, 185. - Physiological study of the cerebral functions by stimulation and extirpation, I88. - Clinico-pathological study of cerebral functions, I9I. - Functional and organic diseases of the brain, I94.

THE idea that functions are localized in different parts of the brain is an old one. It was held by the representatives of the pseudo-sciences of physiognomy and phrenology (phrenology being a branch of physiognomy). The phrenologists believed that they could tell the mental characteristics of an individual from a study of the conformation of his cranium. They did not mean by this that these mental characteristics are determined directly by the shape of the cranium, but that the cranium indicates the nature of the brain where these characteristics are determined. It is true that the cranium may give some slight indications as to the nature of the brain it incloses. But these indications are at best very crude and are likely to be very misleading. So that in course of time physiognomy and phrenology became thoroughly discredited as furnishing scientific data as to the nature of the brain. Reacting from these pseudo-scientific theories it was the tendency of physiologists for a time to deny the existence of cerebral local- 
ization and to believe that each organ in the brain acted as a whole. Thus, according to this theory there would be no localization of function within the cerebrum, but the whole of the cerebrum would be involved each time it functioned. However, recent methods of investigation have furnished a good deal of evidence of cerebral localization, and these data will now be discussed.

\section{Methods of Studying Cerebral Functions}

There are three principal methods of studying the functions of the cerebrum; namely, the anatomical, the physiological, and the clinico-pathological. ${ }^{1}$ The anatomists dissect the nervous system and determine the distribution of the nerve cells and trace the paths of the nerve fibers. From such study many inferences, some of which are quite trustworthy, may be drawn as to the functions of the different parts of the nervous system. For example, if it were known that a certain effector was not connected at all by a nerve with a certain central organ, it would be reasonable to suppose that the effector was not governed in any way by that organ. The nervous system is so complex and, as we have seen in the last two chapters, is so thoroughly integrated, that it is doubtful if there is any effector that is wholly unconnected with any central nervous organ. But if the connection was indirect and very slight, it would be reasonable to suppose that the degree of control of the central organ over the effector was very slight. In similar fashion if an effector was connected very directly and by many

1 Cf. Shepherd I. Franz, On the Functions of the Cerebrum: The Frontal Lobes, in the Archives of Psychology, Vol. I; and The Functions of the Cerebrum, in the Psychological Bulletin, Vol. VIII, No. 4 (April 15, 1911). 
fibers with a central nervous organ, it would be reasonable to suppose that the central organ had a high degree of control over the effector.

Physiological research includes the methods of stimulation and extirpation. By stimulating parts of the nervous system and observing the motor and vascular reactions obtained, functional relations between different parts of the nervous system and effectors may be determined. By extirpating parts of the nervous system and then observing the effect upon the behavior of the individual, inferences may be drawn as to the functions of the parts extirpated. The extirpation method is of chief value for the study of the parts of the cerebrum which are not excited by direct stimuli. The study of pathological conditions in the brain may throw much light upon the problem of cerebral localization. If a lesion of a certain part of the brain is followed by a derangement in the behavior of certain effectors, it is reasonable to suppose that there is a functional relation between that part of the brain and those effectors.

By means of the methods of research described above, a vast amount of data has been gathered with regard to the cerebrum, and I can cite only a few general conclusions from some of the leading investigators.

\section{ANatomy of the Brain}

First let us note a few general anatomical facts. The brain, or encephalon, is developed from three embryological divisions; namely, the anterior, the middle, and the posterior brain vessels or vesicles, which are at the anterior end of the neural tube. The anterior vesicle becomes the fore-brain, or prosencephalon. The middle vesicle becomes the mid-brain, or mesencephalon. The posterior vesicle becomes the rhombencephalon. In the course of embryological development 


\section{The Science of Human Behavior}

a constriction appears in the anterior vesicle and another in the posterior vesicle, so that each of these become divided into two, and there are five secondary brain vesicles in the place of the primary three vesicles. The fore-brain, or prosencephalon, becomes divided into the end-brain, or telencephalon, and the inter-brain, or diencephalon. The rhombencephalon becomes divided into the hind-brain, or metencephalon, and the after-brain, or myelencephalon. The cerebrum includes all of the fore-brain and mid-brain, and the rhombencephalon includes the other parts of the brain. The three primary brain vesicles are at first in a straight line like the rest of the neural tube. But early in embryological development they begin to curve, so that in course of time they assume the rounded form of the brain. ${ }^{1}$

The cerebrum, like the rest of the cerebro-spinal system, is composed of white and gray matter. The white matter is made up chiefly of bundles of medullated axones. The gray matter is composed chiefly of cell bodies and dendrites. It is evident, therefore, that stimuli from receptor sense organs must terminate in the gray matter, and impulses to effector organs must start from the gray matter, while these stimuli and impulses must be carried in large part by the white matter. The cerebral gray substance may be divided into three groups; namely, the cortical, the ganglionar, and the central or ventricular. The cortical gray substance is of most interest to us, for here are determined in the main the actions of the individual which involve psychic qualities.

The cortex of the cerebrum has been described in the following words: - "The exterior surface of the fore-brain is composed of a thin sheet of gray matter varying in thickness from one sixth to one quarter of an inch. That gray matter forms a barklike covering for the

${ }_{1} \mathrm{Cf}$. on the anatomy of the brain: H. E. Santee, Anatomy of the Brain and Spinal Cord, Philadelphia, I907; Johnston, The Nervous System of Vertebrates, Philadelphia, 1906 ; L. F. Barker, The Nervous System, New York, 1899; Wundt, Principles of Physiological Psychology, Vol. I, London, I904; Howell, A Textbook of Physiology, Philadelphia, I906, chaps. IX-XI. 
underlying white substance and is, therefore, called the cortex. It is thrown into irregular elongated folds named convolutions, or gyri, by deep linear depressions, which greatly increase the relative amount of cortical substance. The linear depressions are called fissures, or sulci; and, in consequence of them, the gray substance is increased in bulk to fifty-eight and one half per cent of the entire cerebrum (De Regibus)." 1 This statement that the gray matter constitutes more than half of the cerebrum sounds rather exaggerated when we consider that in the hemispheres the gray matter is only the superficial covering of the cerebrum, but when the ganglionar and ventricular gray substances are added, this statement may be correct.

The white matter of the cerebrum is composed in the adult of medullated fibers. These cerebral fibers form three systems: (I) projection or peduncular fibers, (2) transverse or commissural fibers, (3) association fibers. ${ }^{2}$ The projection fibers are connected only with the motor and sensory areas of the cerebral cortex and are, consequently, motor and sensory in function. The commissural fibers connect opposite sides of the cerebrum. The association fibers connect parts of the same hemisphere. We shall refer again to these systems of fibers after having studied in more detail the cerebral cortex.

\section{Anatomical Theories of Cerebral Localization}

As has already been indicated, the phrenologists formulated a theory of the localization of functions in the brain and in the cerebrum in particular. But their theory was quite untenable in the light of further scientific researches. These functions as localized in the brain they termed "internal senses." Gall distinguished twenty-seven of these, and his pupil Spurzheim increased the number to thirtyfive. Some of these senses were most preposterous, such as the sense of facts, the fear of God, philoprogenitiveness, poetic talent, the impulse of self-preservation, etc. Reacting from those pseudo-scientific ideas, the physiologists

Santee, op. cit., pp. 5I-52.

2 Santee, op. cit., pp. 224 ff. 
tended for a time to the belief that the cerebrum acted as a whole in performing its functions like certain other organs of the body, such as the heart, the lungs, etc. In similar fashion they believed that the other parts of the brain, such as the cerebellum, the medulla oblongata, etc., also performed their functions each as a whole. And some of the experiments of extirpation seemed to confirm this belief, for it was found in some of these experiments that when a part of the cerebrum was extirpated, the cerebral functions were weakened as a whole, but no functions were completely abrogated. But as more knowledge was acquired with regard to the structure of the brain and the course of conduction paths, and as pathological observations were made of the results from local lesions in the cerebrum, the modern theories of cerebral localization were formulated. It goes without saying that these modern theories do not postulate specific centers for any such complex characteristics as did the phrenologists. The determination of each one of those characteristics would require the coöperation not only of many parts of the brain, but of other organs of the body as well.

Wundt says, looking at these theories from the psychological point of view, that the terms "sensation" and "idea" now represent the two fundamental forms of psychical process in the place of the internal senses of the phrenologists. "'Sensation' means in this connexion, any conscious reaction evoked by external sensory stimuli, while 'idea' includes, in accordance with the nomenclature of the older psychology, all kinds of 'memory image.'" On this basis two theories of localization have developed. "From these general premisses, the doctrine of localisation or, as its close relations to the older phrenological theories justify us in calling it, 'modern phrenology' has developed in two directions. In both forms, it is based upon the assumption that the cerebral cortex is divided up into a number 
of sensory centres, in which the excitations brought in along the sensory conduction paths release the specific sensations.... The one form of the doctrine of localisation asserts that the centres of sensation and idea are strictly connected, so that every sensory centre covers both processes, and the entire cortical surface is therefore essentially composed simply of a number of adjacent sensory centres. . . The second form of the localisation doctrine differs from the first mainly by its assertion that the central areas which subserve the colligation of sensations, and therefore also the retention of ideas, the centres, in fact, which underlie the complex psychical functions at large, are spatially separate from the sensory centres, though connected with them by manifold systems of association fibres. It accordingly gives this second order of centres, whose office it is to colligate the various sense departments, the special name of 'association centres'; and we may therefore designate the second form of the localisation theory briefly as the "theory of association centres.' "1

Let us now consider some of the schemes of cerebral localization prepared by anatomists. As has already been indicated, part of the cerebral cortex is devoted to receiving stimuli from receptor organs and sending impulses to effector organs. This part is divided up into several areas which are usually called the sensori-motor or senso-motor areas. Another and larger part of the cortex is devoted to establishing associations between the sensory and motor centers. This part is divided up into what are called the association centers.

Johnston describes the senso-motor areas as follows: - "The afferent or sensory fibers which come to the neopallium ${ }^{2}$ from lower brain centers end in certain regions, and from these regions alone (Flechsig) arise the great majority of the efferent or motor fibers which carry impulses to the motor centers of the brain and spinal cord. Hence these regions are given the name of the sensomotor areas. Three sensory centers are recognized, the visual, the auditory, and the somesthetic areas. The last is the area for general

${ }^{1}$ Wundt, op. cit., p. 289 . $\quad{ }^{2}$ The neopallium is a part of the cerebral cortex. 
bodily sensations. Since motor tracts descending from the visual and auditory fields are not well known, a general area corresponding roughly to the somæsthetic area is commonly known as the motor area. The only way of describing the limits of these areas is by means of the superficial sulci and gyri of the brain." $1 \mathrm{He}$ then describes the location of the somæsthetic area, the visual area, the auditory area, the gustatory area, and the motor area which, he says, "is practically coextensive with the somæsthetic area." These areas form about one third of the cortex. The remaining two thirds is taken up by the association areas. "The remaining two-thirds of the cortex is intercalated between the several senso-motor areas so that each of them is separated by a considerable space from the others. This whole area constitutes, according to Flechsig, the association centers, and it may be divided into three main fields, the anterior, middle, and posterior association fields." 2 Johnston defines the functions of these association areas more specifically as follows: "By association fields must not be understood areas in which the functions of association are carried out without the aid of the sensomotor areas. This would be physically impossible, and the term correlation centers would more truly express the function of the association fields. In other words, the function of the association centers is to correlate the actions of the senso-motor centers." ${ }^{3}$ Each one of the association areas is apparently devoted to establishing connections between the senso-motor areas which it adjoins. This is indicated in the following statement with regard to the functions of each of these areas: "The specific functions of the three association fields are determined in large part at least by their position with reference to the senso-motor areas. The middle association field (island of Reil), situated as it is between the auditory area and that part of the somæsthetic area which receives sensations from the lips, tongue, throat, etc., is chiefly the association center for speech. The anterior and posterior fields have much more complex relations and functions. The posterior field, situated between the somæsthetic, visual, and auditory areas, receives from those areas impressions concerning the external world. The functions of this field are to construct external objects from the several kinds of sense impressions and to form ideas concerning the relations of objects and physical processes to one another and to the self. In a word, the objective relations of the in-

$$
{ }^{2} \text { Op. cit., pp. 345-346. } 2 \text { Op. cit., pp. 349-35r. } \quad \text { Op. cit., p. } 352 .
$$




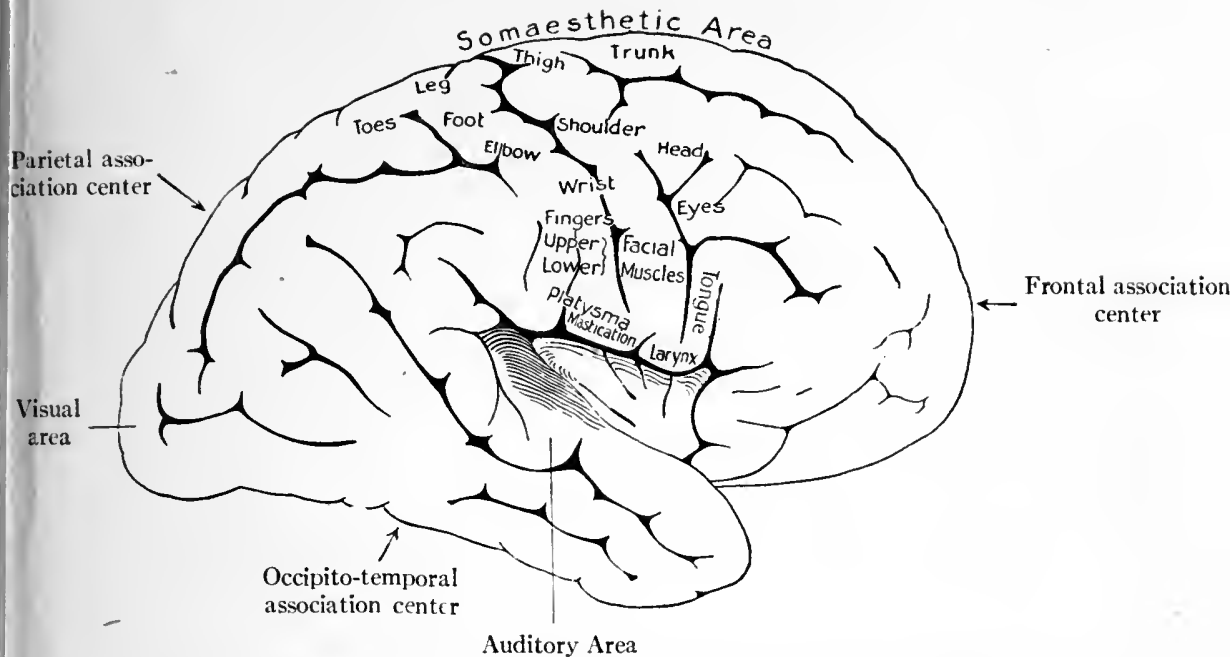

Fig. II. - Diagram of the convex surface of the cerebral hemisphere. (After Morris.)

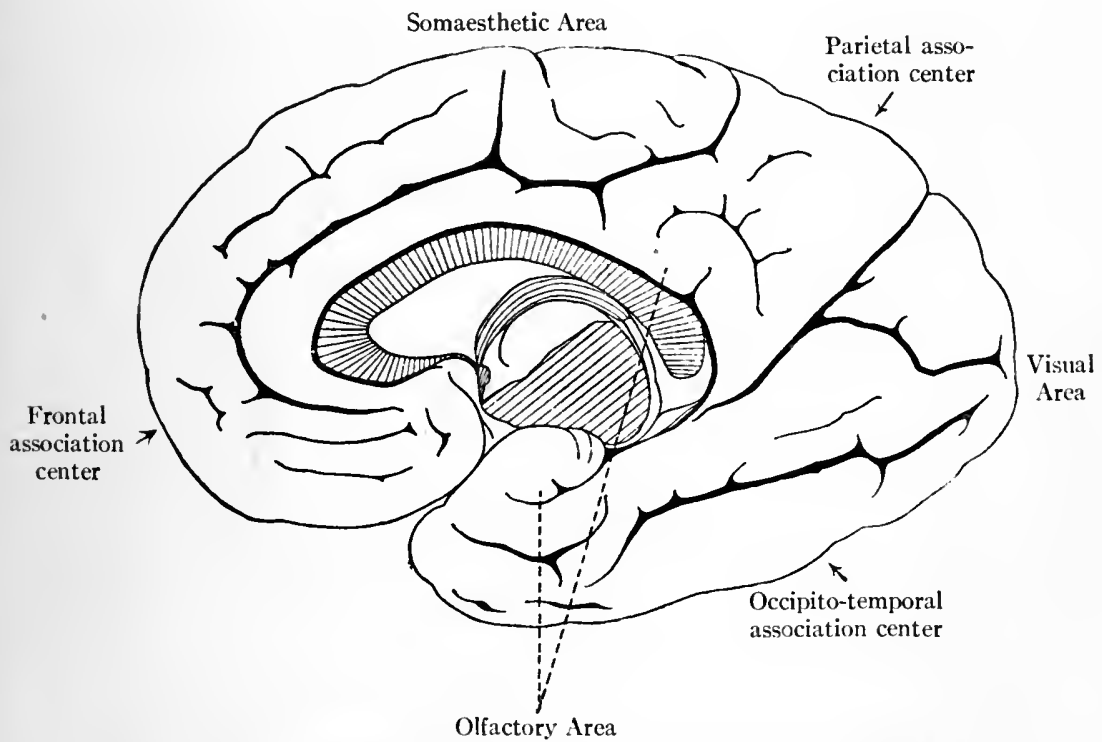

Frg. r 2. - Diagram of the medial surface of the cerebral hemisphere. (After Morris.) 

dividual and all those processes which we commonly call 'intellectual' are the peculiar province of the great posterior association field. . . . The anterior association field lies in proximity to the somæsthetic area, but removed from the other sensory areas. It would receive in common with the posterior area the impressions due to contact with external objects and to the movements of the body, limbs, organs of speech, etc. Although the distant connections of the frontal lobe are not well understood, it seems clear that fibers from the visual and auditory areas are of subordinate importance, while association bundles from the olfactory (and gustatory) centers are next in importance to those from the somæsthetic areas." 1

Santee ${ }^{2}$ gives a description of the cortical areas which is similar to that of Johnston, though the terminology used is somewhat different. He prefaces the description with this general statement. "By repeated observation of the symptoms produced by definite brain lesions and by the anatomical and physiological study of human and lower animal brains, both in the embryonic and adult condition, the cortex has been mapped out into certain definite functional areas. Psychic function undoubtedly is dependent upon the associated activity of a number of cortical areas; but motor, common sensory, and special sensory regions have been outlined with considerable exactness."

The motor area he locates as follows: "The emissive motor area is situated in the anterior wall of the central sulcus, in the posterior one-half of the gyrus centralis anterior and in that part of the paracentral lobule immediately continuous with it. This is the center for ordinary voluntary motion on the opposite side of the body. Axones from this area descend to the nuclei of all motor nerves." This area is divided into four segments; namely, the head and neck, the arm, the trunk, and the leg. He then describes the psychic motor areas: "The psychic motor areas, or areas for educated movements, are located just anterior to the above motor areas, in the an-

$$
{ }^{1} \text { Op. cit., pp. 354-355. } \quad 2 \text { Op. cit., pp. 177-183. }
$$


terior central gyrus and in the contiguous ends of the superior, middle, and inferior frontal gyri. These areas are believed to send their axones to the emissive motor centers in the cortex." These psychic motor areas include centers for the lower extremity, the arm, which is the writing center, and the organs for the voice, which is the motor speech center. The common sensory area is described as follows: "According to Dr. Alfred W. Campbell, the receptive area of common sensation is limited to the posterior wall of the sulcus centralis, including the anterior one-half of the posterior central gyrus and that part of the paracentral lobule which is continuous with it." This area "is probably divided into segments similar to those of the motor area." He then locates the psychic sensory area. "A large portion of the remainder of the parietal cortex probably constitutes a number of centers for the interpretation of common sensory impulses, hence the term, psychic sensory area." The divisions of this area are not known with certainty, but different investigators have believed that they have located the muscular sense and the parts that interpret tactile, pain, and temperature impulses.

The sense of hearing he locates as follows: "The receptive acoustic center is located in the transverse gyri and in that part of the superior temporal gyrus which is continuous with them." The psychic acoustic center is adjacent. The sense of vision is located as follows: "In the cuneus and lingual gyrus is located the receptive optic center for the temporal half of the same retina and the nasal half of the opposite one; perhaps, also, for the macula lutea of both sides." The psychic optic center is near to the receptive one. The senses of smell and of taste are located as follows: "The uncus hippocampi forms the chief cortical center of smell, close to which in the fusiform gyrus is probably the gustatory center (Mills)."

Some of the anatomical characteristics which differentiate these areas are indicated in the following passage: "The olfactory, auditory, visual, common sensory, and motor areas are all distinguished by a definite characteristic histological structure peculiar to each region (Campbell). Medullation of the fibers in these cortical areas occurs at different times; and, according to Flechsig, in the following order: olfactory, tactile and muscular sense, visual, auditory, and gustatory." Certain other centers have been located, at least tentatively, such as the center of intonation, the naming center, the center of equilibration, and the center of orientation. As has already 
been suggested, not all of the centers which have been mentioned have been located with certainty, but further anatomical research aided by the other methods of investigation will undoubtedly increase greatly the number which are known with certainty. These cortical areas are connected with the receptor and effector organs for which they function by the projection fibers which we have already described. "All the above motor, somæsthetic, and special sense areas are provided with projection fibers which connect them with definite muscle groups and surface regions and with the organs of special sense. Large parts of the cerebral cortex possess no projection fibers; they are believed to be associative in function."

Next he describes the three association areas beginning with the anterior: "According to Flechsig, that part of the frontal cortex which is anterior to the motor region determines the temperament and individuality of the person; and, as Mills declares, is the center of inhibition, self-control, attention, "concentration, volition. It is the center of 'the abstract concept.' J. S. Bolton says of this association center that 'it is the last part of the cerebrum to be developed, and is the first to undergo dissolution; it is underdeveloped in amentia of all grades and atrophied in dementia, according to its degree.' 'It possesses the highest (mental) function' (Brain, Vol. XXIX). The posterior association center, composed of those portions of cortex situated between the sensory region of the equatorial zone, in front, and the visual cortex of the occipital lobe, behind, determines the intellectuality of the individual. To acquire knowledge of the external world is thus the function of the posterior association center. Mills calls it the center of 'the concrete concept.' It includes three psychic areas - the common sensory, auditory, and visual. Flechsig regards the island (of Reil) as the middle association center. Lesions in it are associated with paraphasia."

These association areas must have many fibers which connect them with sensory and motor centers. These fibers are described as follows: "They are situated within or beneath the cortex, the various parts of which they serve to unite. Association fibers become medullated and actively functional only as mental effort and education gradually develop them. So far as the brain is concerned, education consists, first, in the development of the functional centers of the brain ; and, second, in the establishment of lines of rapid communication between them. The short association fibers are the more 
numerous and are very important. They unite contiguous parts of the same gyrus and associate together adjacent gyri." 1 The number of these fibers and the connections they establish are indicated. "The short association fibers are almost infinite in their connections. They connect the receptive and psychic sensory areas, and their interruption on the left side causes inability to interpret the sensations, called mind-blindness, mind-deafness, stereognosis, etc. Again, those short fibers also associate the psychic with the psychic-motor, and the psychic-motor with the emissive-motor centers. In this manner the writing center is connected with the motor center for the upper extremity, and the speech center with the motor centers for the lips, tongue, etc.: breaking of the former connection on the left side destroys ability to write, agraphia; and aphasia results, if the latter connection is broken. Besides these and many other connections of associated centers, the short fibers join together the various parts of each cortical area." 2

Before leaving the subject of the anatomical study of cerebral localization we must touch upon the subject of the neural connections of these cerebral centers with their receptor and effector organs.

Santee has discussed this subject well in his chapter on the "Tracing of Impulses." 3 He classifies the neural paths by which these connections are established as follows: "I. Efferent, or motor. II. Afferent, or sensory - general and special sense. III. Reflex." These paths are direct when they do not pass through the cerebellum, and indirect when they do pass through it. The cerebellum, then, performs the function which we have already discussed and which is indicated in the following citation: "The cerebellum is an important relay in the indirect motor and indirect sensory paths. In response to impulses received from skin, muscles, tendons, joints, and viscera, it is also believed to originate impulses which coördinate muscles and maintain equilibrium. Moreover, according to Russell, each cerebellar hemisphere exercises an important inhibitory function, through the brachia conjunctiva, upon the opposite side of the cerebrum." 4

$$
\begin{aligned}
& \text { 1 Op. cit., p. } 236 . \quad 2 \text { Op. cit., pp. } 237-238 . \\
& \text { Op. cit., p. } 242 .
\end{aligned}
$$


I have not the space to discuss at length these paths, but will indicate the two types of sensory paths, namely, the general and special. "The sensory paths conduct two varieties of impulses, viz., general and special. The impulses originate in the end-organs of the cerebral and spinal nerves, and by those nerves are conveyed to the cerebrospinal axis, through which they reach the proper cortical area in the cerebrum." The general sensory paths perform the following functions: "General sensation is the function of the sense of touch. This sense has four important subdivisions - the tactile sense, muscular sense, pain sense, and temperature sense. Stereognosis is only an associated interpretation of all the impulses of the sense of touch, and not a subdivision of it. Tactile sensations appear to be most elemental and, according to Mills, may be conducted by all common sensory nerve fibers. Other common sensations seem to require some specialization, as yet not understood, in their conducting media ; and pain and temperature impulses pursue a path entirely distinct from that followed by impressions of the muscular sense." The special senses are those of smell, sight, hearing, and taste. "Impulses producing the sensations of smell, sight, hearing, and taste are carried from the respective organs of sense to the brain by the following nerves: the olfactory, the optic, the auditory, and the glossopharyngeal and intermediate nerves."

The reflex paths Santee classifies as follows: "Reflex arcs are formed: (I) by the sensory and motor fibers of spinal nerves, associated in the gray matter of the cord; (2) by the sersory and motor fibers of cerebral nerves, which are connected in the brain; (3) by afferent spinal fibers connected by the ascending fibers of the medial longitudinal bundle, with efferent cerebral fibers; and (4) by afferent cerebral and efferent spinal nerve fibers, the two being associated by the anterior longitudinal bundle, the ponto-spinal tracts, the fasciculi proprii, the spinal tract of the fifth nerve, the vestibulo-spinal tract, the solitary tract, etc." These classes of reflexes he calls (I) spinal reflexes, (2) cerebral reflexes, (3) spino-cerebral reflexes, (4) cerebrospinal reflexes.

The preceding has been a brief discussion, based in the main on writings of two authorities, of the anatomical researches with regard to cerebral localization. Before leaving the subject we must touch upon the physiological 


\section{The Science of Human Behavior}

and clinico-pathological investigations of the same subject. These two lines of investigation are closely related to each other. For that matter, these two are also closely related to the anatomical method of research. The results attained along each line throw light upon the results attained along the other lines, so that it is impossible to separate the three from each other to any great extent.

\section{Physiological Study of Cerebral Localization}

As has already been indicated, in the physiological study of cerebral localization two methods of research are used; namely, stimulation and extirpation. Electricity is frequently used in stimulating the cerebrum. It is evident that if upon stimulating a certain part of the cerebrum a motor reaction follows, it is reasonable to suppose that this motor function is localized in this part of the cerebrum. In similar fashion a deduction may be made from the response to any stimulation as to the function which is localized in that part of the cerebrum. It is, however, well to check the results from this line of investigation by means of other methods of research. By extirpating a part of the cerebrum and observing what functions disappear, we can determine tertatively what functions are localized in that part of the cerebrum. As has already been indicated, however, there is always some uncertainty in such a deduction. In the first place, the surgical shock from the operation may have affected other parts of the cerebrum in such a way as to prevent them from functioning, so that more functions disappear than can be attributed to the part which has been extirpated. In the second place, other parts of the cerebrum may compensate for the part which 
has been extirpated, so that the functions localized in that part may not disappear. That is to say, these functions may be taken up by other parts of the cerebrum, so that it will not be apparent what functions were localized in the part which has been extirpated. Such compensation can take place only when a function is not absolutely localized in one part of the cerebrum, but may be performed by other parts as well. It is evident, therefore, that in the case of extirpation, also, the results of this line of investigation should be checked by researches along other lines as well. The extirpation method is especially valuable for the study of the parts of the cerebrum which are not excitable, or, rather, which do not display noticeable effects upon stimulation.

Clinico-pathological investigations have thrown much light upon cerebral localization. Where there has been derangement in the behavior of an individual it has sometimes been possible by means of trephining operations in the living and by means of autopsies in the dead to locate the lesion which was the cause of the derangement. Thus conclusions could be arrived at as to the localization of the functions which have been deranged. This method of investigation has peculiar value for the study of cerebral localization in man. Frequently the patient can give detailed information as to the nature of the derangement, which is of great value, especially in the study of localization in the association areas.

Let us note a few illustrations of these methods of investigation. Franz, who has been one of the leading investigators of the frontal lobes of the cerebrum, gives as follows the results from some of his stimulation and extirpation experiments, though he has used material from pathological cases as well:- 
" $A$. In monkeys and cats the frontal lobes are normally employed in the formation of simple associations.

" $B$. When the frontal lobes are destroyed, recently formed habits are lost.

" $C$. The loss of the associations is not brought about by lesions of other portions of the brain.

" $D$. The loss of the associations is not due to shock, for lesions of other parts of the brain are not followed by loss of habit, nor does the anæsthetic and loss of blood, etc., produce loss of associations.

" $E$. Unilateral lesions of the frontal areas are not followed by a loss, but there may be a slowing or retardation of the motor response.

" $F$. Habits once lost after removal of the frontals may be relearned. The relearning takes about as long a time as if the animal were learning a new association.

" $G$. Only newly formed habits seemed to be lost after such lesions. Long-standing habits seemed to be retained.

" $H$. The emotional condition of the animal remains the same after as before the removal of the frontals." 1

From these results he draws the following conclusions: "From the clinical and experimental results of others as well as those which have been recorded in the present paper I conclude that the frontal lobes are concerned in normal and daily associational processes and that through them we are enabled to form habits and, in general, to learn." 2

In a later writing on the same subject Franz gives the following summary of the knowledge as to functional localization in the brain: "Although the consideration of the known functions of the cortical areas has been necessarily brief, sufficient indication has been given that the motor and the sensory (hearing, touch, vision) cortical mechanisms are understood and localized. Opposed to this definiteness there is a vagueness in regard to the functions of the association areas. Some few facts are known, but there appears to be more or less haze about the subject, and writers tend to avoid it. Following is a summary of the known facts regarding the two large association areas: (a) in the frontal association area are located centers of motor speech and writing; $(b)$ in the posterior association area are centers for what clinicians call the understanding of auditory and visual speech; (c)

I S. I. Franz, On the Functions of the Cerebrum: The Frontal Lobes, in the Archives of Psychology, Vol. I, New York, 1908, p. 63.

2 Op. cit., p. 64 . 
the frontal regions are clearly associated with the production of movements, especially those of a complex character; $(d)$ in the posterior association area (or in the closely associated intermediate postcentral cortex) is an area for the understanding, through the medium of the skin and motor sensations, of the character of objects; $(e)$ the results of my work on monkeys and cats indicate that both the frontal and the posterior association areas are concerned in the formation of simple sensori-motor habits." 1

Later on he indicates the special functions of the frontal portions of the cerebrum. "Evidence is at hand to show that the frontal areas have a more direct connection with the motor areas than do the posterior. The variations of reflex movements concomitantly with variations in frontal lobe activity have been mentioned. . . . It is by a continual process of adjustment that man has attained his place in the world, and the varieties of activities so characteristic of him is made possible through the frontal regions. The direct motor responses of an animal need no large amount of association elements, but the indirect reactions of man require an amount of coördination that is supplied by the association cells. There is the increased use of the hands, of tools, of the legs, and of other mechanisms for propulsion, requiring new adjustments and new associations; and the impulses to act are paralleled by a similar number of impulses to check activity, or tending to check the primary, reflex-like impulses." 2 Then he indicates the special functions of the posterior portions of the cerebrum. "The posterior areas are, from the accounts given above, clearly more closely allied with the sensory spheres. The large size of this region in intellectual men is only one indication of this, but the sensory aphasias and other similar processes that are located here by clinicians confirm this view." ${ }^{3}$

Clinico-pathological Study of Cerebral Localization

As has already been indicated, the clinico-pathological and the physiological methods of investigating cerebral localization are closely related. As a matter of fact, a

1 S. I. Franz, On the Association Functions of the Cerebrum, in the Jour. of Phil. Psych. and Sci. Meth., Vol. VII, No. 25 (Dec. 8, I910), p. 679.

2 Op. cit., p. 681.

${ }^{3}$ Op. cit., p. 68I. 
pathological lesion and an experimental extirpation may have the same effect, because in each case a certain part of the cerebrum has been destroyed or has been cut off from the rest of the nervous system so that it can no longer perform its function. In other words, the experimenter has done intentionally what disease or accident may do quite unintentionally. A vast number of data have been gathered with regard to the relations between pathological mental conditions and forms of behavior and abnormal conditions of the brain.

The significance of these data for cerebral localization is well stated by Johnston in the following passage: "Thus, in case of any disease or injury which produces a lesion of a part of the cerebral cortex, one or more bodily functions may be interfered with, and the study of many cases has shown clearly that the functions affected depend upon the specific regions of the cortex injured. A sufficient number of facts of this sort have been collected from clinical observations, post-mortem examinations, and surgical operations to render fairly certain and accurate the determination of the area of the cortex involved in case of a brain tumor, degeneration of a cortical substance, or other cause of disturbance. If the patient shows symptoms of disturbance in the functions of sight, hearing, bodily sensation, or voluntary movement including speech, certain specific areas of the cerebral cortex may be pointed out as the seat of the disease, and in the case of bodily sensation or movement certain subdivisions of the cortical area may be assigned to certain parts of the body." 1

Santee indicates in the following passage the different results from lesions, on the one hand, of the motor or sensory cortex, and, on the other hand, of the association centers: "Destructive lesions of parts of the motor or sensory cortex cause merely loss of certain motions and sensations represented by those parts, but ablation of association centers disconnects the sensory, the psychic, and the motor regions and causes aphasia, agraphia, change of temperament, impairment of the so-called moral and intellectual faculties, etc. Ablation of the 


\section{Cerebral Localization}

visual psychic center or auditory psychic center produces mind-blindness in the former and in the latter mind-deafness." 1

Bolton describes how the cerebral cortex evolved from three primary cell laminæ or layers, and shows how pathological conditions arise from the sub-evolution or degeneration of these laminæ. "In cases of mental disease grading from idiots and imbeciles through various types of non-demented and partially demented lunatics to the gross dement, great differences in the degree of evolution and dissolution of the cortex exist; and these, considered from the general aspect, follow the order of normal development. In amentia the condition is one of sub-evolution to different degrees, and in dementia the laminæ suffer in the reverse order to that of their evolution, the most affected being the latest developed, and the least affected being the earliest developed." 2 He goes on to indicate how the causes of these pathological conditions are localized in the cerebral cortex. "The degree and type of these differences vary according to the region of the cortex, whether this be a projection area, a zone of special association, or the prefrontal (higher associative) region. In the case of the visuo-sensory area, the prominent features are poor evolution of the pyramidal and polymorphic laminæ and specialization of the granule or receptive lamina. There also exist individual variations in the degree of evolution of the granule or receptive lamina, and in that of the pyramidal or associative lamina, which individual variations bear no relationship to the degrees of amentia or dementia which exist in individual cases. In the case of the visuo-psychic zone, the important feature, apart from an especially good evolution of the granule or receptive lamina, is a marked degree of individual variation with regard to the depth of the pyramidal or associative lamina. This individual variation is independent not only of the existing grade of amentia or dementia, but also of the individual variation in this lamina which occurs in the visuo-sensory area." 3 At the end of this monograph he emphasizes the need for the study of these cases in order to determine the causal relations between these pathological conditions and the cerebral areas. " $I$, therefore, trust that the definite relationship, which exists between the clinical evidences

${ }^{1}$ Op. cit., p. 183 .

2 J. S. Bolton, A Contribution to the Localization of Cerebral Funchon, Based on the Clinico-Pathological Study of Mental Disease, in Brain, Vol. XXXIII, Part I 29 (1910), p. I05.

${ }^{3}$ Op. cit., p. 105. 
of amentia and dementia during life and the corresponding indications of cerebral sub-evolution and dissolution which are found after death, will in the future receive fuller recognition than has hitherto been bestowed upon it. The preliminary acceptance of this general truth, followed by the combined clinical investigation of individual cases and macroscopic and microscopic study of the cerebra of these, is, I am convinced, essential as a scientific basis for the rational study of mental disease." 1

But while many brain diseases can be localized in the cerebrum, there are other diseases which are caused by a general abnormal or pathological condition of the brain. Krafft-Ebing calls these diseases psychic or mental diseases. "There can be no doubt that the disturbances of psychic functions as they occur in insanity are the expression of changes in the organ that, under normal conditions, makes possible the occurrence of the psychic processes. Thus, the psychic disease proves the existence of a disease of the cerebral cortex; and, since circumscribed cortical disease (focal lesions) can occasion only symptoms of defect referable to the diseased portion of the cortex, the psychic abnormality can only be conditioned by a diffuse change in the cerebral cortex." 2 These diffuse changes he attributes in large part, if not wholly, to disturbances of nutrition. It may, however, be questioned if it is correct to limit the term psychic or mental disease to those diseases which are caused by a general pathological or abnormal condition of the cerebrum. There certainly are other diseases whose causes are in part or wholly localized which may be termed psychic or mental diseases as much as those whose causes are not localized.

1 Op. cil., p. I3I.

2 R. von Krafft-Ebing, Textbook of Insanity, Philadelphia, I905, pp. 20-2r. 
This distinction is the same as that which is frequently made between the so-called functional and the organic diseases of the brain. Many of those who make this distinction are persons who are using pseudo-scientific and more or less unscientific methods for the cure of these functional diseases. These diseases are supposed to be caused in some mysterious way without any anatomical changes taking place in the brain, and they are supposed to be cured quite as mysteriously by methods which are not said to have any anatomical effect upon the brain. It cannot be emphasized too strongly that the functional as well as the organic diseases have an anatomical basis and that they differ from each other only in that the changes are not so great and are much more numerous and more widely distributed in the case of functional diseases. Krafft-Ebing makes this distinction between functional and organic diseases, and yet he seems to recognize their likeness. "Like many other diseases of the central nervous system without demonstrable post-mortem lesions, the majority of psychic diseases seem to be for that reason functional; to be the result of molecular changes - a disturbance of nutrition. The conception of many psychoses as being functional diseases must not, however, be given too wide an application, and thus encourage neglect of investigation of the pathologico-anatomic foundation of the psychoses. It must not be forgotten that in many forms of mental disease pathologico-anatomic lesions are found which are almost identical." 1 And again he speaks as if functional diseases have an anatomical basis. "Thus, from an anatomic standpoint, mental disease may be defined as a diffuse

$$
1 \text { Op. cil., p. 2r. : }
$$




\section{The Science of Human Behavior}

disease of the cerebral cortex consisting of changes which may vary from mere alterations of cortical nutrition to gross changes of structure, especially inflammatory and degenerative in character." 1 It would perhaps be better if the distinction between functional and organic diseases could be abandoned entirely, because in the last analysis all diseases are both functional and organic.

1 Op. cit., pp. $2 \mathrm{I}-22$. 


\section{CHAPTER XI}

\section{THE NATURE OF INSTINCT}

Mistaken conceptions of instinct, 197. - Instincts as congenital tendencies to action, 198. - The relation of instinct to tropism and reflex action, 198. - The adaptive nature of instincts, 205. - The structural basis of instincts, 206. - The hereditary nature of instincts, 208. - Instincts as characterizing species, 208. - The degree of permanency of instincts, 209. - Variations in instincts, 209. - Reënforcement and inhibition, 2 ro. - Combinations of instincts, 212. - The degree of perfection attained by instincts, 213. - Instinct and consciousness, 214. - The relation between instincts and emotions, 220. - Definitions of instinct, 222. - An instinct as an inherited combination of reflexes, 226 .

IN the discussion of the behavior of the lower organisms earlier in this book was described one important form of behavior, the tropism. In the description of the nervous system was described a second important form of behavior, the reflex action. We now come to a third important form of behavior, the instinct, or, more specifically, the instinctive action.

The terms "instinct" and "instinctive" are very frequently and usually mistakenly used. For example, in his most recent reading the present writer has noted the mention of humanitarian, prophetic, criminal, moral, religious, patriotic, benevolent, and political instincts. And yet it is almost certain that not one of these instincts exists. In fact, the popular use of these terms merely implies more or less definite modes of action which are preceded by very 
little or no deliberation. This is why instinct is frequently opposed to reason. Furthermore, it is frequently assumed that the behavior of animals is instinctive, while that of men is rational. It was generally believed in the past that the instincts were divine in their origin. For example, the English writer Addison said that instinct is to be explained "as an immediate impression from the first Mover, and the Divine energy acting in the creatures." 1 Presumably, in accordance with this theory, man has been divinely endowed with the higher faculty of reason. It goes without saying that this pious theory no longer has any standing in the light of modern science, and it is a grave error to limit instinct to animals and reason to man. As a matter of fact, man shares most of the instincts with the animals, while some of the animals may possess a certain amount of reason. Let us therefore search for a more accurate conception of instinct.

\section{Relation of Instincts to Tropisms and Reflexes}

We may say, to begin with, that instincts are congenital tendencies to more or less definite modes of action. It is evident that this definition would include tropisms and reflex actions, for, as we have seen, these forms of behavior are congenital and are more or less definite. And it is held by some writers on this subject that instincts are tropisms, while others believe that they are reflex actions. We shall therefore have to consider these theories first before we can arrive at a more limited and detailed definition of instinct.

Loeb, whom we have come to know as the great champion and exponent of the tropism theory, insists upon identifying

${ }^{1}$ Quoted in G. J. Romanes, Animal Intelligence, 5th edit., London, I892, p. II. 
tropisms, reflexes, and instincts with each other. He contends that instincts consist largely if not entirely of tropisms, and that instincts cannot be distinguished from reflexes. For example, he says: "It is evident that there is no sharp line of demarcation between reflexes and instincts. We find that authors prefer to speak of reflexes in cases where the reaction of single parts or organs of an animal to external stimuli is concerned; while they speak of instincts where the reaction of the animal as a whole is involved (as is the case in tropisms)." 1 In similar fashion Folsom identifies these three forms of behavior with each other: "We have already emphasized that an instinct is a reflex act or a combination of reflex acts. The same fact may now be stated in these words: an instinct is a tropism or a combination of tropisms." ${ }^{2}$ T. H. Morgan seems to regard tropisms and instincts as identical. For example, he speaks as follows: "Many of the so-called instincts of animals have been shown in recent years to be little more than direct responses to external agents." 3 $\mathrm{He}$ goes on to discuss as instincts various of these direct responses which are tropisms.

It is evident that the first two of the three writers who have just been cited extend the idea of the reflex action to organisms without a nervous system. In an earlier chapter were discussed the reasons for restricting the use of this term to animals with a nervous system. Of the writers who do so and then identify instincts with reflex actions, the most distinguished is Herbert Spencer. In

1 Comparative Physiology of the Brain, and Comparative Psychology, New York, 1900, pp. $7-8$.

2 J. W. Folsom, Entomology, Philadelphia, 1906, p. 361 .

Evolution and Adaptation, New York, I903, p. 382. 
the first place, he defines reflex action as follows: "Under its simplest form, Reflex Action is the sequence of a single contraction upon a single irritation," but "Reflex Action proper is exhibited only when we ascend to creatures in which there exist nerves and muscles." 1 Then he defines instinct as follows: "Instinct may be described as compound reflex action." ${ }_{2}$ He goes on to say that "no clear line of demarkation can be drawn between it and simple reflex action."

After having written the preceding chapters, I hardly need to say that I believe that there is strict continuity between all these different forms of behavior and that the more complex forms are built up from and based upon the simpler. The question, therefore, of what meanings we are to give to these terms is purely a matter of terminology, to be determined by considerations of expediency. In an earlier chapter I have stated my reasons for thinking that it is preferable to restrict the word reflex to the actions of animals with a nervous system. It is evident that if we extended the term to the actions of all animals, we should be calling all forms of behavior reflex, and in that case it would mean no more than to say that all forms of behavior are mechanically determined, in which case it would have little utility. For similar reasons it is, I believe, better not to identify instinct with tropism, for if we did so we should be giving to the word instinct a very broad meaning. Indeed, in the last analysis, it would then include every form of behavior, and consequently would have no utility in distinguishing different forms of behavior. I shall therefore restrict the use of the term instinct to the behavior of ani${ }^{1}$ Principles of Psychology, Vol. I, New York, 1895, p. 427.

2 Op. cit., p. 432. 
mals with a nervous system. The next question is as to whether instinct is to be identified with reflex action.

One group of writers have contended that instincts and reflex actions are to be distinguished from each other on the ground that an instinct involves a mental element, while a reflex action does not. Romanes is one of the best-known representatives of this group. He defines reflex action as follows: "This non-mental operation of the lower nerve centers in the production of apparently intentional movements is called Reflex Action." 1 Later on he states that instinct is reflex action with a mental element added. His definition of instinct is as follows: "Instinct is reflex action into which there is imported the element of consciousness." 2 I shall discuss the question as to whether or not an instinct necessarily involves consciousness further on. in this chapter, while the whole subject of consciousness will be discussed more or less fully in a later chapter. The conclusion which will then be reached will be that instinct does not necessarily involve consciousness, so that this cannot be regarded as a distinguishing mark between reflex action and instinct.

Is there, then, any other distinguishing mark between reflex action and instinct? It seems quite evident that it is the tendency of neurologists to-day to regard all action which is determined by the nervous system as reflex. Practically all the actions of the higher animals are determined by the nervous system. Certainly this is true of all actions which have an outward manifestation, while very few if any actions inside the body are not determined by the nervous system. A possible exception may exist

1 Animal Intelligence, London, 1892 , p. $3 . \quad 2$ Op. cit., p. 17. 
in the case of the muscles of the heart, which seem to be independent of the nervous system sometimes, as in the embryo before the nervous system develops. ${ }^{1}$ This may also be true of the visceral muscles. ${ }^{2}$ It is evident that in accordance with this conception of reflex action all instinctive action must also be reflex, for all instinctive actions involve the nervous system. Upon accepting this conception of reflex action, the only distinction which can be made, if any can be made, between reflex action and instinct is that an instinct involves more than one reflex action. This is in accordance with Spencer's definition of instinct as compound reflex action. According to this conception of instinct, then, any form of behavior which involves more than one reflex action is instinctive. Some writers, however, such as Lloyd Morgan, ${ }^{3}$ contend that for behavior to be instinctive it must involve the organism as a whole. If this be true, more than two reflexes would as a rule, if not always, be required to constitute instinctive behavior, for it is doubtful if any two reflexes alone would involve the organism as a whole. To the above might be added the statement, which so far as I know has not been made by any other writer on this subject, though many may have intended to imply it, that instinctive action is a form of behavior which has an outward manifestation, for there is probably no one who would call an internal process instinctive.

If, then, the above considerations are correct, the only difference between a reflex and an instinctive action is

1 Cf. G. H. Parker, op. cit., p. 339 ; C. S. Sherrington, The Role of Reflex Inhibition, in Science Progress, No. 20 (April I9II), p. 585.

2 Cf. W. H. Howell, Textbook of Physiology, Philadelphia, rgo6, pp. 53-54.

Habit and Instinct, London, 1896, pp. 7, 27. 
one of degree. An instinct would simply be a compound or complex of reflexes. There might still be difference of opinion, however, as to the degree of complexity needed to constitute an instinct. For example, some writers call the sucking action of the lips displayed by newly born infants an instinct. But Lloyd Morgan and the others who contend that instinctive action must involve the behavior of the organism as a whole could not regard this sucking action as being more than a reflex, for it does not involve the whole organism.

Some writers, however, who have admitted that an instinct is made up of reflexes have still contended that there is a fundamental difference between an instinct and a reflex. For example, Hobhouse has taken this position. $\mathrm{He}$ attempts first to distinguish between the two in this fashion: "Each particular act may be described without obvious violence as reflex, but the whole is an adaptive combination of reflexes in which the combination is as important as each separate act." I It is not, however, clear how this constitutes an absolute distinction between the two. Certainly, so far as adaptation is concerned, there can be no such distinction, for reflexes may be quite as adaptive as instincts. Instincts, therefore, still remain compounds or complexes of reflexes. He passes on, however, to a further distinction. After describing the instinctive fashion in which a hen cares for her chicks, and insisting that this is not reflex, he says: "There seems to be at least some permanent state corresponding to what we call maternal feeling, or the parental instinct which dominates the hen's actions throughout, and without which the various

1 Mind in Evolution, London, I90I, p. 53. 


\section{The Science of Human Behavior}

reflexes would not be discharged by their appropriate stimuli." 1 This passage seems to indicate that he thinks that an instinct involves a state of feeling, and that this is what distinguishes it from a reflex action. Apparently, therefore, his conception of an instinct is like that of Romanes, which has been discussed.

A little further on he characterizes instinct again as follows: "Where there is by heredity a certain setting determining reflexes in a special way, there is an instinct which is distinguishable from a compound reflex. The instinctive act is no longer one which follows with perfect uniformity from a certain stimulus. It follows from that stimulus only if it is appropriate to the setting of the organism at the time. We have indeed already noted that reflexes may be adaptable to organic conditions. It is on such adaptable reflexes that instinct rests, and its business is precisely to shape them aright." ${ }_{2}$ He himself recognizes in this passage that reflexes are adaptive, like instincts. So far as being hereditary is concerned, there can be no distinction between the two. So that the only distinction apparently resides in this "setting," which he says determines the reflexes which constitute an instinct. Just what this setting is, he does not indicate, but it is presumably the state of feeling to which he has referred earlier but has not described. Hobhouse's description is therefore at best an ambiguous one, and his conception of an instinct seems to be like that of Romanes. As I have already indicated, this conception will be discussed later in this chapter.

What, then, are we to say as to the relation between reflex action and instinct? If we identify instinct entirely 
with reflex action, the term would no longer have any utility in distinguishing different forms of behavior, for it would be synonymous with reflex action. Inasmuch as practically all the actions of higher animals are determined by the nervous system, all the behavior of these animals is almost, if not entirely, reflex in its character. It is therefore impossible to apply the term instinct to behavior which is not reflex. The only way of utilizing the term is therefore to apply it to a special group of reflex actions. I have already noted the distinguishing features of this special group. In the first place, they must be actions which have an outward manifestation. In the second place, there must be more than one of these reflex actions, and these actions must be so combined and related to each other that they will work towards a unified end. That is to say, each must be adapted not only by itself, but also with relation to all the others. In other words, instincts appear in the course of the integration of the behavior of the organism which is effected by the nervous system and which has been described in an earlier chapter. When we regard instinct in this fashion, the mystery and obscurity in which it has so frequently been shrouded at once disappears, and the nature of instinct becomes very simple and clear.

Whether, then, we shall use the term depends upon the question as to whether it is worth while to give a special name to certain of the higher stages in this process of integration. It is perhaps worth while to do so, because the use of this term emphasizes the part played by external, selective forces in guiding and, to a certain extent, determining the course of this process of integration. It is a significant fact that neurologists very frequently do not use the 
word instinct even when discussing instinctive actions. For example, I doubt if Sherrington in his admirable account of the integrative action of the nervous system which has been cited uses the term instinct once, even though many of the integrated forms of reflex action which he describes are instinctive. And it is probably wise of these writers not to use the term, for by so doing they emphasize the reflex character of the behavior of the higher animals. But these scientists usually have all their attention centered on the nervous system of individual organisms and pay no attention to the selective forces which are eliminating those organisms whose reflexes do not adapt them to their environment, while they preserve those whose reflexes do so adapt them. The idea of adaptation is very closely associated with the word instinct, and it may therefore be profitable to use the term in describing integrated reflex actions whose character has been determined in part by external selective forces.

Having identified instinct with the forms of behavior of the whole organism which have been integrated by the nervous system, it is evident that in an earlier chapter, when discussing the integrative action of the nervous system, I was describing one aspect at least of the origin and evolution of instincts. I shall, therefore, not repeat this description, but will discuss briefly certain other aspects of the origin and evolution of instincts.

\section{Structural Basis OF INSTINCT}

It must be evident by this time that instincts are based upon structure and that the relation between instincts and structure is the same as the relation between structure and 
physiological activities. James apparently has this in mind when he says that instincts "are the functional correlatives of structure." I Structure is therefore for instincts what the "action system" is for the behavior of the lower organisms, as we have seen in an earlier chapter. The origin and evolution of instincts is therefore analogous to that of structure. The fundamental principles of morphological evolution have been discussed in earlier chapters. We have seen that structural forms evolve either as the result of the accumulation of slight variations which are preserved because they have utility, or as the result of mutations of more or less size which appear independent of utility and which therefore may or may not prove to be useful. If an instinct was determined by a structural form which had evolved as a result of the accumulation of slight variations, it would probably make its appearance slowly and would be useful from the start. If, however, an instinct was determined by a structural form which was the result of a sudden mutation, it also would probably appear suddenly and would not necessarily be useful at the start. So far as I know, no such sudden appearance of an instinct as the result of a mutation has been observed. But if it be true, as I have contended, that instinct is based upon structure, and if structural forms may result from sudden mutations, there is every reason for believing that instincts may appear in this sudden fashion. It may be contended by some that such new forms of behavior would not be instincts if they were not useful, for many writers have insisted upon the utility of instincts as an essential characteristic. But while in the long run instincts are cer-

1 William James, Principles of Psychology, New York, 1896, Vol. II, p. 383. 
tain to be useful, for otherwise they would be eliminated, an instinct may not be useful at the start when it is the result of a sudden mutation, and an instinct which has been useful may lose its utility.

\section{Characteristics of Instinct}

It must now be evident that instincts are hereditary just as the structures upon which they are based are hereditary. It has been contended by some that instincts originate in modes of behavior acquired by the individual which are usually called habits, or in memories which may also be reduced to terms of habit which are inherited. It is evident that we cannot accept this theory of the origin and the hereditary nature of instinct unless we accept the theory of the inheritance of acquired characters, and, as we have seen in a previous chapter, there is little ground for believing this theory.

The next question which arises is as to the number of individuals to which an instinct must belong. Some definitions of instinct state or imply that an instinct is a form of behavior which characterizes all or most of the members of a species. It is true that an instinct tends to become a specific character, because if it proves to have great utility, the individuals possessing it will be preserved, while those which do not possess it will be eliminated. But at its inception an instinct cannot very well belong to a whole species unless it in itself, or rather the structural form upon which it is based, marks the beginning of a new species. If an instinct is the result of a sudden mutation, it will at first belong to but one individual. Then it will be transmitted to the immediate descendants of this indi- 
vidual, and if it proves to have great utility, it may become a specific character in the manner indicated above.

It is thought by many that instincts are permanent and unchanging in their character. But it is evident that they can be no more permanent than the structural forms upon which they are based. We have seen that variations and mutations cause new instincts to come into existence. In similar fashion variations and mutations cause changes in instincts already in existence because they change the structural basis of these instincts. Sometimes these variations and mutations cause instincts to disappear gradually or suddenly. These changes in instincts are hereditary in their character. Are there any changes in the instinctive tendencies of the individual which are not hereditary in their character? If there are any such changes they must be due to structural changes which cannot be inherited. Let us see whether there are any such changes.

A simple reflex action is not likely to be changed, because its structural basis is fairly simple, and changes in it are not likely to take place. But the structural basis for the unified behavior of the whole organism which arises out of a group of reflexes which have been integrated together by the nervous system and which we call instinctive is much more complex, and the possibility of changes taking place in this structural basis is correspondingly greater. It is therefore in the nervous system by which this integration of reflexes has been effected that these changes in the structural basis usually take place. And the nervous system is so delicate and so complex that changes in it which are very slight in bulk may have a great effect upon the behavior of the organism. These changes may, however, take place in other 
parts of the organism. For example, one of the most important and permanent of the instincts is the sexual instinct. The structural basis of this instinct is to be found in part in the sexual organs and in part in the nervous system. If the operation of castration is performed in which the sexual organs are removed in part or entirely, the instinctive sexual tendency will disappear in part, if not entirely. If such a change is not observed, it must be due to the fact that a habit has become so firmly established on the instinctive foundation that it persists in its full strength even though the structural basis for the instinct has been in part removed.

But let us see what changes appear in instinctive reactions as a result of changes in the nervous system. As we have seen, an instinctive act is one that is performed without any previous training or experience the first time that the appropriate stimuli affect the organism. Consequently there can be no knowledge or foresight of the consequences of the act by the individual before the act is performed. This is why it is so unfortunate that instinctive actions are so frequently spoken of as purposive or teleological in their character. They may be adaptive and therefore useful, but inasmuch as in a purely instinctive act the individual does not foresee the consequences of the act, there can be no purpose on the part of the individual. The situation is changed, however, when the act is repeated, for then the factor of experience enters in to affect the behavior. The individual now has a knowledge of the consequences of the act and will be influenced accordingly. If the consequences have been pleasant, the tendency to perform this act will be strengthened. If the consequences 
have been unpleasant, the tendency to perform this act will be weakened and perhaps in course of time inhibited entirely. Thus it is that through associations established in the central nervous system instincts are reënforced or inhibited.

Let us see what are the principal ways in which such reënforcement or inhibition takes place. As we have seen, an instinctive action is stimulated by any one of a group of objects which are more or less alike and which starts off the series of reflexes which constitute the instinct. An instinctive action may be stimulated in an individual a number of times by the same one of this group of objects. Thus the habit of responding to this one object may become firmly established, so that when the individual is subjected to the stimulus of other objects of this group it will be prevented from responding to these because of the habit which has become established in favor of the first one. ${ }^{1}$ Thus the tendency to respond to the first object of the group will be greatly reënforced, while the tendency to respond to the other objects of the group will be inhibited. It is easy to see that such reënforcement and inhibition arise out of paths of association which have become established in the central nervous system.

An instinctive reaction may be stimulated by the image of one of the group of objects which ordinarily arouse it. The image of an object arises out of an association center or group of association centers which is necessarily connected with the sensory center which that object stimulates. If this association center or group of association centers is also connected with the motor center which governs the instinctive reaction, stimulation of this center or group of

1 Cf. W. James, op. cit., p. 394. 


\section{2

centers may be sufficient to arouse the motor center. Thus an instinctive reaction may be aroused by the image of an object, even though the individual has not been subjected to the stimulus of the object itself. In similar fashion an instinctive reaction may be stimulated by another object, or the image of another object which is connected by paths of association with the object which ordinarily arouses the instinctive reaction, and also with the motor center which governs the instinctive reaction. ${ }^{1}$ The neural basis for the fact that the same instinctive action can be aroused by stimuli from several sensory and association centers is probably indicated in the principle of the common path which has been discussed in an earlier chapter. It will be recalled that this principle is to the effect that a final or efferent neurone is a public path "common to impulses arising at any of many sources of reception."

The form of behavior which an instinctive reaction takes may vary greatly as the result of habit based upon experience. This is peculiarly true of man, whose behavior is controlled to a certain extent by intelligence and is influenced by imitation. Such variations in the form of behavior manifested in an instinctive reaction are most likely to take place in the case of instincts which ripen slowly, so that intelligence has an opportunity to influence the form the instinctive reaction is to take. ${ }^{2}$

Different instinctive reactions may be stimulated at the same time and then inhibit each other in part or entirely, or they may be combined in forms of behavior in which each reaction becomes somewhat modified.

1 Cf. W. McDougall, Introduction to Social Psychology, Boston, 1909, pp. 32-4a

2 Cf. McDougall, op. cit., pp. 40-43. 
The individual variations in instinctive reactions which have just been briefly described are caused in large part or entirely by the establishment of paths of association in the brain which connect new centers with the motor centers for instinctive reactions. There is a certain type of instinct in which a lack of uniformity in the instinctive reactions may seem to indicate individual variations. These are the transient instincts which appear temporarily. The appearance and disappearance of these instincts is, of course, due to changes in the structure of the individual. But inasmuch as these changes are common to the whole group, which is characterized by a transient instinct, this group usually being a species, the variation in the instinctive reaction can hardly be spoken of as an individual variation.

It is a popular idea that instincts are perfect. But the variations in instincts which have just been discussed indicate that very frequently, if not always, they are imperfect. This raises the question as to what constitutes perfection. It is hardly necessary to say that science does not recognize any absolute metaphysical or moral standard of perfection. If it recognizes any standard at all, it must be a relative one. In the organic world it is utility in the struggle for existence. Thus instincts are perfect to the extent to which they are useful in the struggle for existence of the organisms which possess them. Can it be said, then, that instincts are ever perfect in accordance with this standard? It is true that selection is always at work preserving the instinctive reactions which are useful and eliminating those which are injurious and, to a certain extent, those which are not useful. But selection has probably never succeeded in making an organ or an organic character as adaptive 
and therefore as useful as it might have been. Consequently every organ and organic character has fallen short of perfection according to our relative standard, and always there has been room for improvement. This is true even of so highly developed an organ as the human eye, as was shown by Helmholtz. It is therefore hardly conceivable that any instinct was ever perfect in the sense that it was perfectly adapted to the needs of the organism. Certainly it would be more difficult for an instinct to become perfectly adapted than for most organic characters, because of the complexity of the series of reflexes which constitutes an instinctive reaction. Furthermore, even if an instinct did become approximately perfect, it would probably not remain so for very long, for the needs of the organism would be likely to change soon, thus necessitating changes in the instinctive reaction. Lloyd Morgan has indicated the character of instinct very well in the following statement: "Instinctive behavior is practically serviceable on the occasion of its first performance." 1 That is the truth about instinct,-it tends to be practically serviceable rather than theoretically perfect.

\section{Instinct AND Consciousness}

We must now take up the question of the relation of instinct to consciousness. As has been noted, certain writers have contended and do now contend that an instinctive action always involves a mental element, and by this mental element they usually mean consciousness. This question, like so many others, depends to a large extent upon the way

1 The British Journal of Psychology, Vol. III, Part 3 (October, 1910), p. 225. The italics are mine. 
in which we define certain terms. If a sufficiently broad definition is given to such words as mind and consciousness, no one would deny that an instinctive action always involves a mental or conscious element. Such would be the case if it were contended, as it is by some, that mind or consciousness is the essential vital characteristic of all organisms. In that case a mental or conscious element would necessarily enter into every organic activity. But if we are to limit the meaning of these terms somewhat narrowly, we may be able to prove that a mental or conscious element is not necessarily present in every instinctive action. Let us note briefly some of the theories that contend that an instinct necessarily includes a mental or conscious element.

I have already referred to Romanes' theory that an instinct always involves a mental element, while a reflex action does not, and that this mental element in instinct is consciousness. We must determine next what he means by consciousness. In his chapter ${ }^{1}$ on this subject he refuses to define consciousness in what he believes to be its character as a mental or psychic phenomenon, but he describes the physiological basis of consciousness. He contends that consciousness arises when there is enough delay between a stimulus and the response of the organism to it to permit of its appearance. In the case of a simple reflex he believes that the time is not long enough to permit consciousness to appear, but in the case of an instinctive action he believes that there is time enough and that the consciousness always does appear. Before commenting on this conception of consciousness, I wish to speak of the other reasons which Romanes apparently has for insisting upon consciousness as an

1 Mental Evolution in Animals, New York, 1898, chap. VI. 
essential part of instinct. Again and again in his extensive writings on instinct he speaks of instinct as hereditary memory. Furthermore, he classifies instincts as primary and secondary according to their modes of origin. Primary instincts result from the natural selection of actions "which, although never intelligent, yet happen to have been of benefit to the animals which first chanced to perform them." 1 Secondary instincts result from the hereditary transmission of habits of actions originally intelligent. George Henry Lewes considered these instincts cases of "lapsed intelligence." It is evident that throughout this discussion Romanes is implying a belief in the inheritance of acquired characters, a theory of which he was an ardent supporter. Memory, according to the usual meaning of that term, is an acquired character, and Romanes seems to be using it in this sense. ${ }^{2}$ While he does not expressly say so, he seems to imply that the primary instincts result from habitual modes of action which have been accidentally acquired and then preserved for hereditary transmission by natural selection. He states explicitly that the secondary instincts result from the hereditary transmission of acquired habits. So that it is evident that his conception of instinct is based throughout upon the hypothesis that acquired characters are transmissible by heredity. $\mathrm{He}$ implies that the consciousness of their acts possessed by past generations is somehow transmitted so that even the first time an instinctive act is performed it is accompanied

1 Op. cit., p. 178.

2 "Hereditary memory, or instinct, belongs to what I have marked off as the second and third stages of conscious memory in the largest acceptation of the term - the stages, that is, where, without any association of ideas, a present sensation is perceived as like or unlike a past one."-Op. cit., pp. II5-116. 
by consciousness. As we have seen in an earlier chapter, there is very little reason for believing in the hereditary transmission of acquired characters, while practically all the evidence is against it. Consequently most of Romanes' reasons for thinking that instinct always involves consciousness fall to the ground at once.

Returning to his conception of the nature of consciousness described above, let us see if that furnishes any ground for this belief. There can be little question that some delay between stimulus and response is necessary for the appearance of consciousness. But it may be questioned whether this is the only requisite condition. I shall not discuss this question here, because it will be discussed more or less thoroughly in a later chapter. But even if a certain amount of delay between stimulus and response were the only requisite condition for consciousness, it is doubtful if the delay is long enough in the case of many instinctive actions to give rise to consciousness. The delay in an instinctive action which involves several reflexes is longer than in a simple reflex because it involves a more complicated nervous adjustment. But even in an instinctive action the response is frequently very immediate, so that there is little chance for the appearance of consciousness. This is very likely to be the case the first time an instinctive action takes place before any habits or other inhibiting forces have developed to delay the response. But this may be the case, also, when an instinctive act has been many times repeated, so that habits and other acquired characters reënforce it and lessen the time between stimulus and response. Consequently, even in accordance with the very simple criterion of consciousness which Romanes proposes, it may be questioned 
whether instinct always involves consciousness, while it becomes still more questionable in accordance with a more complex criterion, as, for example, the one which I shall outline in a later chapter. It seems quite evident, therefore, that Romanes' reasons for believing that consciousness is an essential element in instinct were very poor.

It would take too long to discuss all the theories of instinct which have insisted upon a mental or psychic element as an essential part. I have already commented upon Hobhouse's theory, which seems to be similar to that of Romanes. In his recent discussion of instinct, which in many respects is most excellent, McDougall offers the following definition of instinct: "We may, then, define an instinct as an inherited or innate psycho-physical disposition which determines its possessor to perceive, and to pay attention to, objects of a certain class, to experience an emotional excitement of a particular quality upon perceiving such an object, and to act in regard to it in a particular manner, or, at least, to experience an impulse to such action." 1 It is evident that this definition is heavily weighted with psychological implications; much more so, in fact, than the great majority of definitions of instinct, and is, therefore, very different from the much simpler biological conception of instinct in which we are most interested.

Let us see what these psychological implications are. In the first place, he says that an instinct is a "psychophysical disposition." We have seen that very different meanings are given to such terms as "psychic," "mental," and "conscious." If it is contended that all organic matter 
possesses psychic qualities, then it is easy to believe that an instinct is in part at least psychic. If, however, we are to limit the meaning of the term more narrowly, an instinct may not always be psychic. We must therefore determine how complex are the "psychic" characteristics which McDougall attributes to instinct. He says that "there is every reason to believe that even the most purely instinctive action is the outcome of a distinctly mental process," and that it "can only be fully described in terms of the three aspects of all mental process - the cognitive, the affective, and the conative aspects; that is to say, every instance of instinctive behavior involves a knowing of some thing or object, a feeling in regard to it, and a striving towards or away from that object." 1 It is evident that he believes that even the simpler instinctive actions involve such highly developed mental phenomena as knowledge, a complex feeling in the form of an emotion, and conscious effort towards an end. But the preceding discussion certainly has been sufficient to show that instinctive actions, which are series of reflexes which have been integrated by the central nervous system, appeared long before any one of these mental phenomena had developed, so that no one of these is an essential part of an instinct. If, then, any psychic phenomena are always involved in instinctive actions, they must be more simple than the ones enumerated by McDougall. As has been indicated, my own conception of the psychic is to be outlined in a later chapter. All I need to say here is that I believe that psychic phenomena arise out of the adjustments accomplished by the central nervous system in the interval between the reception of a stimulus by a 
sense organ and the sending of an impulse to a motor organ. But these adjustments must be relatively complex to give rise to such psychic phenomena, and it is almost if not quite certain that the adjustments involved in many of the simpler instinctive actions, like those of the simple reflexes, are not sufficiently complex to give rise to psychic phenomena.

To continue this discussion of the psychological implications in McDougall's definition, we may note in the second place that an instinct "determines its possessor to perceive" and in the third place "to pay attention." The implication seems to be that the perception and attention involved are conscious, and later on he asserts that instinctive actions are conscious. In any case it is doubtful if these terms can be used legitimately where there is no consciousness, so that he is again assuming the presence of psychic characteristics where they are not necessarily present.

In the fourth place, he says that an instinct involves "an emotional excitement of a particular quality." It is certainly true that many of the more complex instincts are accompanied by an emotion which is peculiar to the instinct it accompanies. But it is a grave error to assert this of all instincts. In fact, this assertion is quite contradictory to the prevailing theory as to the origin of emotions which was first formulated by Lange and James. According to this theory, emotions result from modes of behavior or bodily changes of some sort. It is therefore reasonable to suppose that if an instinctive action is always accompanied by a certain emotion, the emotion is caused by the action. The action must therefore have come into existence first in order to give rise to the emotion. It is possible that in 
certain cases where an instinct has appeared suddenly as the result of a mutation of considerable extent, the emotion has appeared at the same time as the instinctive action. But in the great majority of cases the emotion must have come after the instinct. McDougall repudiates the JamesLange theory of emotions in certain respects, but he seems to accept it in its essential points, so that it is hard to understand why he insists upon an emotion for every instinct. In fact, it is hard to believe that $\mathrm{McD}$ ougall really thinks that every instinct possesses all the psychic characteristics he enumerates in this definition. It seems as if he must intend to apply this definition only to some of the most highly developed instincts. For these instincts this definition may be accurate, and these psychic characteristics should be recognized, for, as McDougall says, "if we neglect the psychical aspect of instinctive processes, it is impossible to understand the part played by instincts in the development of the human mind and in the determination of the conduct of individuals and societies; and it is the fundamental and all-pervading character of their influence upon the social life of mankind which alone gives the consideration of instincts its great practical importance." 1

It may appear as if $I$ am laying too much emphasis upon this point that an instinctive action does not necessarily involve any psychic, mental, or conscious processes. But from the point of view of this book such emphasis is essential. It is one of my objects to show that the so-called psychic characteristics have been caused by the different forms of behavior, if indeed these characteristics are not in themselves forms of behavior, which possibility I shall 


\section{The Science of Human Behavior}

discuss later. As instinctive action is one of these forms of behavior which have given rise to psychic phenomena, it is very important to emphasize its priority to these psychic phenomena. But by doing so I do not mean to imply that instinctive action is not very frequently intermingled with and accompanied by psychic phenomena, and, as we shall see, there is an evolutionary continuity between instinctive behavior and intelligent and rational behavior which involves the highest psychic qualities.

\section{DefintTIONS OF Instinct}

We have now discussed briefly the most important aspects of the general theory of instincts and are in a better position to define instinct accurately and in detail than we were at the beginning of this chapter. I shall, however, summarize the conclusions so far reached and discuss certain other definitions before attempting to formulate one of my own.

The first point discussed in this chapter was the relation of instinct to tropisms and reflexes. It was shown that some writers identify instincts with these other forms of behavior. But to do so is to destroy the utility of two of these terms. It is therefore preferable to limit their meanings somewhat so that they will have utility in distinguishing between different forms of behavior. I limited tropism to the reactions to external forces of animals without a nervous system. The term reflex I limited to the reactions of animals with a nervous system. The term instinct $I$ applied to an integrated series of reflexes.

We saw that instincts are based upon structural forms which are congenital. Consequently, instincts are in- 


\section{The Nature of Instinct}

herited. An instinct may be manifested by but one individual or by a very few, as when it is caused by a sudden mutation in structure. But usually an instinct characterizes a whole species. Instincts vary in accordance with changes in the structural forms upon which they are based. Instincts are not perfect, even according to a relative standard of perfection, but they are usually adapted and consequently are usually "practically serviceable." An instinct does not necessarily involve mental, psychic, or conscious qualities or phenomena.

Any accurate definition of instinct must be in accordance with the above statements with regard to instinct. Let us see if any definition of instinct has been devised which is satisfactory both as to accuracy and as to the degree of detail with which it describes instinct. I have already mentioned and criticized a few. It would be impossible to quote all of the large number which have been suggested. I will mention but a few more which are more or less typical, some of which are among the most successful which have been devised.

James' definition of instinct is as follows: "Instinct is usually defined as the faculty of acting in such a way as to produce certain ends, without foresight of the ends, and without previous education in the performance." 1 It is evident, to begin with, that this is a very vague definition and might cover a tropism or a simple reflex action as well as an instinct. Furthermore, it makes instinct necessarily purposeful in its character which, as we have seen, is very objectionable. This is a typical example of a large number of definitions which are vague and emphasize too strongly 


\section{The Science of Human Behavior}

the adaptive and purposeful character of instinct. It is rather strange that James should have formulated such a definition as this, for his conception of instinct seemed to have been based upon the nervous system and to have been quite clear-cut. This definition also does not state that instinct is hereditary, though this may be implied in the statement that instinctive action does not require previous education.

Lloyd Morgan has suggested the following definition: "From the biological point of view - instincts are congenital, adaptive, and co-ordinated activities of relative complexity, and involving the behaviour of the organism as a whole." 1 Here again it is not stated explicitly that instinct is based upon the nervous system, though the statements that instinctive activities are coördinated and that they are of relative complexity may be meant to imply it. It is also stated that instincts are adaptive. Aside from these points this is a fairly good definition and is less vague than most of them. As we have already seen, there may be difference of opinion as to whether an instinctive action must necessarily involve the organism as a whole. However, it is probably better not to insist that an instinctive action must involve the whole organism, for there are many congenital activities which involve only a part of the organism, but which are in every other respect exactly like the instinctive activities which involve the whole organism.

Spencer's definition of instinct as "compound reflex action," which has already been quoted, is quite inadequate and has been misleading to many who thought that it identified instinct completely with reflex action. But it em- 
phasized the fact that instinct is based upon the nervous system and was therefore a long step in the right direction and influenced many later definitions.

Perhaps the best definition of instinct which has so far been formulated is the following: "An instinct is an inherited reaction of the sensori-motor type, relatively complex and markedly adaptive in character, and common to a group of individuals." 1 While it is not stated explicitly, the use of the phrase "sensori-motor" implies that instinct is based upon the nervous system. It may, however, be questioned whether an instinctive action is always of the sensori-motor type. Earlier in this chapter has been discussed the possibility of an instinctive reaction being stimulated by the image of an object which usually stimulates it or at first stimulated it. Such a reaction would be of the ideo-motor type. Furthermore it is stated that instinct is adaptive, which, as we have seen, is not necessarily the case. It is also stated that an instinct is common to a group, whereas we have seen that an instinct may belong to but one individual. So that it is evident that even though this may be the best definition which has so far been formulated, it has many faults.

\section{A New Definition of Instinct}

I have now cited enough examples to show that no one of the definitions of instinct which have so far been formulated is satisfactory. A good definition would, in the first place, accord with the facts and, in the second place, would be sufficiently detailed to distinguish instinct from the other

I In the article on.Instinct, signed by J. M. Baldwin, G. F. Stout, and C. Lloyd Morgan, in the Dictionary of Philosophy and Psychology, edited by J. Mark Baldwin, New York, rgor. 
main types of behavior. What, therefore, should such a definition include? In order to distinguish instinct from tropism, it must indicate that instinct is based upon the nervous system. In order to distinguish instinct from reflex, it must indicate that an instinct is made up of an integrated series of reflexes. In order to distinguish an instinctive activity from an internal physiological process, it must indicate that an instinctive activity is an external activity of the organism. I wish, therefore, to propose the following definition of instinct:-

An instinct is an inherited combination of reflexes which have been integrated by the central nervous system so as to cause an external activity of the organism which usually characterizes a whole species and is usually adaptive.

It seems to me that this definition is accurate in every respect and that it is sufficiently detailed to distinguish instinctive behavior from the other main types of behavior. 


\section{CHAPTER XII}

\section{THE NEURAL BASIS OF INSTINCT}

The cerebrum and instinct, 228. - The cerebellum as the principal nervous mechanism for instincts, 228. - Localization of instincts in the spinal cord, medulla, and cerebellum, 230. - Impulsive instincts, 230.-Chain instincts, 231. - Analysis of the nesting instinct of solitary wasps, 233.

IT is evident that according to my definition of instinct the mechanism of instinct must be studied in the nervous system. I believe that much of the writing on instinct has been of little or no value because this has not been done. No complete explanation of an instinctive action has been made until the nervous mechanism by means of which an external object has caused this action has been described. In order, therefore, to explain these instinctive actions, we must turn to the physiologists and neurologists. Unfortunately, however, as has been indicated in an earlier chapter, what is known with regard to the functions of the different parts of the central nervous system is still very meager as compared with the number of phenomena to be explained. It is impossible as yet to trace the nervous mechanism of most of the instinctive actions, especially the more complex ones, so that those who wish to explain instinctive actions fully must wait for physiology and neurology to furnish them more data. Consequently I shall discuss only very briefly the nervous mechanism of instinct and shall then pass on to a discussion of the external forces 
at work in the evolution of instinctive actions and shall describe some of the principal instincts, especially those which are of special importance to man.

\section{NeURal Mechanism of Instincts}

In a preceding chapter was given a brief summary of what is known as to the different kinds of reflex arcs and as to the functions of the different parts of the nervous system. We have seen that some reflexes are simple arcs on the spinal level. Other reflexes on the spinal level are connected with each other by loops so that they affect and influence each other. Still other arcs pass through the brain. Many of the reflexes on the spinal level could not be called instinctive because they do not cause an external activity of the organisms, or because they are too simple. This is true, also, of some of the reflexes which pass through the brain. But some of the reflexes on the spinal level are instinctive and probably still more of those which pass through the brain.

The reflexes which reach the brain pass through different parts of it, and the question may be raised as to what parts of the brain are passed through by the reflexes which form instinctive actions. We have seen in a preceding chapter that the brain consists of four parts - the medulla oblongata, the pons, the cerebellum, and the cerebrum. The function of the medulla and the pons are in the main to connect the other parts of the brain with each other and with the spinal cord. The cerebellum seems to be the organ for coördination in space and for the coördination of voluntary movements. Inasmuch, therefore, as many movements are determined through the cerebellum, it is probable that many 
instinctive actions are controlled by the cerebellum. The cerebrum, or at least the cerebral cortex, is the seat of the association centers, so that all of the higher mental faculties reside in the cerebrum. It is therefore not likely that many of the instinctive actions are controlled by the cerebrum. Indeed, it has been asserted by some that no instinctive actions are controlled by the cerebrum. For example, the well-known neurologist, Edinger, has made this assertion in the following passage: "All sense impressions and movement combinations belong to the palaëncephalon. ${ }^{1}$ It is able to establish simple new relations between the two, but it is not able to form associations, to construct memory images out of several components. It is the bearer of all reflexes and instincts." ${ }_{2}$ He goes on to state that the function of the neëncephalon or cerebrum is to form associations, thus furnishing the nervous basis for intelligent behavior. $\mathrm{He}$ thus draws a very sharp line between instinctive and intelligent behavior by implying that the neëncephalon has nothing to do with instinct and that the palæëncephalon has nothing to do with intelligence. However, if this is what he means, he is probably wrong, for it seems quite certain that the cerebrum has something to do with instinct, while it is quite possible that the rest of the brain has something to do with intelligence, as we shall see in a later chapter. With regard to the connection between the cerebrum and instinct, "F. W. Mott and Pagano have given reasons for thinking that the emotions and instincts are organized in the optic thalami and corpora striata." 3 It has been

1 Under this term Edinger includes all of the brain, aside from the cerebrum.

${ }^{2}$ Ludwig Edinger, The Relations of Comparative Anatomy to Comparative Psychology, in Jour. of Comp. Neurology and Psychology, Vol. XVIII, 5 (Nov. 1908), p. 444.

'Carveth Read, Instinct, in the Brit. Jour. of Psychology, Vol. IV, Part I (May, IgrI), p. 9 . 
found that the cry of distress, which is a very simple instinct, is located in the posterior corpora quadrigemina. Some if not all of the emotions, which are closely related to the instincts, are located in the cerebrum. For these reasons it is hard to believe that the cerebrum has nothing to do with instinct. To do so would be to make the break between instinct and intelligence altogether too great.

\section{Impulsive ANd Chain Instincts}

However, it is undoubtedly true that the cerebrum does not have as much to do with instinct as the other parts of the central nervous system, but is more particularly the organ of intelligence. The instincts are localized in the main in the spinal cord, in the medulla, and in the cerebellum. Some of these instincts are very simple and are manifested in a single external action. This does not mean, however, that these actions do not involve a good many reflexes. These instincts are sometimes called impulsive instincts, and it seems plausible that they are localized in the spinal cord. This, however, is not true of all of them, as, for example, the cry of distress which has been mentioned. Other instincts are much more complex and involve a series of actions. It is probable that most, if not all, of these instincts are localized in the brain. These instincts may be complex because a single external stimulus arouses a reflex which is connected with a number of reflexes, each of which determines an external action, so that a series of such actions take place. Sometimes, however, these instincts are complex because the first action brings the organism under the influence of a new stimulus which causes the second action, and so forth. It may be questioned whether such a series 
of actions could be called a single instinct in strict accordance with our definition of an instinct, since they seem to be a series of separate instincts. Such a series of instinctive actions is sometimes spoken of as a chain instinct, and inasmuch as such actions are inevitably bound to follow each other, and as they are coördinated with each other, it may be legitimate to speak of such a series as an instinct.

In the light of the preceding chapters on the nervous system it ought to be easy to understand the evolution of an impulsive instinct and of a complex instinct, which is made up of a series of actions which are determined by reflexes which are connected with each other in the central nervous system. In the case of these instinctive actions or series of actions, the actions follow close upon the stimulus, so that the connection of cause and effect is very evident. A study of the nervous system shows what the nervous mechanism of these types of instincts is, though we may not be able to trace the mechanism of each particular instinct. External forces are at work selecting out the reflexes which are useful and suppressing those which are harmful, so that these instincts are sure to be more or less adaptive.

But it is more difficult to understand the so-called chain instincts because the causal connections are not so evident and because a long series of actions, sometimes more or less separated from each other in time and place, are so evidently leading up to some end that it is hard to believe that they are not directed by intelligence. This is true of many instincts, some of which are directed towards the preservation of the individual and others to the reproduction of the species. An example of the first kind is where an animal stores up food during the summer to feed upon during the 
winter, while an example of the second is the making of a nest. It has frequently been thought that such behavior is in imitation of others. But this theory has been disproved many times by segregating a young animal from its kind before it has had the opportunity to observe such behavior, and then finding that the instinct still manifests itself under favorable conditions. As has been indicated, it has also been thought that such behavior is intelligent. But a careful analysis of the facts in these cases and a study of the anatomical and physiological characteristics of these animals, combined with a knowledge of the nature of intelligence in general, shows in most of these cases that the behavior could not possibly be intelligent. This will become more evident in a later chapter on intelligence.

In the case of every chain instinct we start with a reflex which is stimulated by an external stimulus. This reflex may have, and in all probability has, been derived from a tropism. The action which results from this reflex may bring. the organism under the influence of an external force which stimulates a new reaction. Or the first reflex action may cause a physiological change in the organism which stimulates a new reaction. Thus it is that a series of actions may become causally connected with each other. But such a series, thus determined by accident, as it were, would not necessarily have any utility. External selective forces, however, are always at work eliminating those which are harmful and sometimes, also, those which are merely useless, and preserving those which are useful so that the actions and series of actions which persist are usually adaptive. It seems almost incredible in the case of the more complex of these instincts that so high a degree of adapta- 
tion could have been attained without any consciousness of the end on the part of the animal. But when we consider that in most of these cases the instincts have existed through thousands of generations, we can see that the selective forces have had enough time to accomplish their work of adaptation. ${ }^{1}$

\section{Nesting Instinct of Solitary Wasps}

Read has given an excellent analysis of one of these chain instincts in his discussion of the nesting instinct of the solitary wasps. Hé has made use of data furnished in the main by Mr. and Mrs. Peckham, but also by Fabre, Lubbock, and other observers. As an example of such an analysis I will quote from his article $^{2}$ at considerable length.

"In attempting to explain the origin and development of these nesting instincts in solitary wasps, I shall assume the general principles of Natural Selection; namely, that variations of behaviour that are advantageous to the species may be inherited, and accumulated by inheritance, and fixed: so that in course of time very complex activities may, through the survival of those individuals that inherit them, and the failure in competition of individuals less well-endowed, become characteristic of the species as a whole. How useful these nesting habits are is shown by an interesting fact. The species of solitary wasp keep up their numbers century after century, age after age, although each female wasp has (compared with most insects, most fish, and even with many mammals) very few offspring - lays very few eggs - less than a score (as well as I can

1 Cf. C. O. Whitman, Animal Behavior, in the Wood's Holl Biological Lectures, Boston, 1899. Whitman gives suggestive analyses of the evolution of various instincts as based upon structural organization. He analyzes, among others, the instincts of keeping quiet and rolling into a ball in worms and insects, of pouting and tumbling in pigeons, of incubation in birds, etc.

2 Carveth Read, Instinct, especially in Solitary Wasps, in the British Journal of Psychology, Vol. IV, Part I (May, I9I I), pp. I-32. 
judge); and this suffices, in spite of many parasites and other enemies. The fact illustrates the general rule of animated nature, that the greater the care taken of offspring, the fewer they are. . . .

"But this nesting instinct is a chain instinct, a series of totally different actions, and to explain it we must consider each step, and also the order in which the steps occur. In the first place, then, we observe that (I) to hide an egg in a hole or other shelter is plainly useful ; (2) so it is to hide or cover up the opening of the hole ; (3) to lay food by the side of the egg, or the egg by the side of the food, is useful, even if there is no nest; (4) to bring more food is useful, if it enables the larva to attain a better growth, or development or potentiality, before the pupa stage; (5) to kill living food, or paralyse it, is useful, that it may not injure the egg or young larva ; (6) to inspect the nest from time to time is useful, in spite of actual shortcomings; (7) to explore the neighbourhood and to identify the spot are useful actions, if the egg and larva are afterwards to be provided for; (8) to return (or 'home') is useful. ...

"Such actions, being useful and immediately useful, will, if they occur in any individual, be perpetuated and tend to become specific; but, in the second place, how do they occur? Let us begin with the making of the nest. To dig a hole in the ground, or in the stump of a tree, or in the stalk of a shrub, or to build a mud cell, as some wasps and some bees do, without knowing what is to be done with it (for plainly they cannot know) - is not this an extraordinary operation? To understand it we must show how it may arise from simpler actions more commonly performed by animals, especially by insects, if possible by hymenoptera, and particularly wasps. We may assume that the peculiar actions characteristic of wasps have been differentiated from a common ground. To find a shelter of some kind for itself or its progeny is an action common to most kinds of the higher animals and to very many insects. Amongst wasps it sometimes takes the form of creeping into a crack in a cliff, or wall (since walls have come into existence), or into a hole in a tree, whether the crack or hole has been made by the ordinary wear and tear of nature, or by some other animal. .

"It has often been objected to the theory of evolution that we never see any species in process of changing form or colour; and the same objection might be urged against the evolution of instincts. It depends on an illusion similar to that implied in the term 'fixed 
stars': we cannot see the stars move, but we can calculate the direction and velocity of the movement of very many of them from facts that can be seen. So no doubt species and their instincts are always changing, but much too slowly for us to notice it within the limits of our short lives. We can, however, sometimes find in natural history, without appealing to embryology, evidence of the changes that have probably been undergone, and may sometimes find a condition of things that seems to imply that a change is now in progress. In such a condition, perhaps, are these instincts I have just described. When a wasp catches its prey before digging a nest, the simplest supposition is that, at first, the prey was left meanwhile upon the ground. It was an improvement upon this when prey was first hung upon a plant until its grave was ready, but not so great an improvement as quickly to exterminate the other practice; and so we still see them existing side by side in the life of the same species. Naturalists who live 50,000 years hence may find that the more careless practice has been entirely lost, or only occurs by atavism in idiotwasps. But, further, the whole double process of either capture and digging, or digging and capture, may be in a state of change: the latter seems to be the commoner; and since it gives greater safety and economises time and energy, it may be gradually exterminating the former course.

"To understand the matter we must try to find how both processes arose. To begin with the case in which capture precedes digging. The capture and killing, or paralysing, of prey in order to lay an egg upon it, is itself a complex process, which must have had a history. We know two simple cases: first, the depositing of eggs upon animals already dead, as by the Blow-fly (Calliphora erythrocephala), and by carrion-beetles (Necrophorus), and by parasitic wasps (Cerophales and Pomp. subviolaceus); and, secondly, the depositing of eggs upon living prey. The latter course is adopted by many flies; and amongst wasps by various species of Ichneumonida, which lay their eggs upon living caterpillars on whose juices the larvæ feed, and by some Braconide, a closely allied family; and Pompilus trivialis oviposits on living spiders. Since a living caterpillar upon which an egg has been laid is still exposed to the attacks of other parasites and enemies, it may give greater safety to kill it; and this is shown to be probable by the comparatively great number of eggs deposited by Ichneumonidee and Microgaster. But much greater safety is obtained if the 
prey is not only killed, but also hidden in a hole or cell. Necrophorus buries the carrion on which its eggs are laid; and parasitic wasps lay eggs on victims already hidden, or about to be hidden, by other wasps. With one utility depending upon another, the combination of killing or paralysing the prey with the hiding of it is not more improbable than the combination (say) of imitative colouration with imitative flight in some butterflies. . . .

"To return to the nesting instinct: why do wasps seek any shelter for their eggs; why construct cells, or creep into holes or crannies? Do they foresee that their progeny have enemies; do they understand danger and safety? We cannot suppose so much. Probably to explain this matter we must fall back upon primitive tropisms phototropism, or thermotropism, or stereotropism. These impulses in such highly evolved creatures as the wasps, may date from remote ancestors in an age before our wasps had become wasps, and may remain active in existing species under conditions in which they are still useful and so far as they are useful. Wasps love sunshine and warmth; shun cold and wet; as the shadows of the afternoon lengthen, nearly all of them seek some sort of shelter, some being content with leaves or grass, others requiring more substantial protection. ...

"But granting an original tropism as the basis of nest-making, we are still required to explain why at a certain time the behaviour originally determined by this tropism becomes effective, contrary to the animal's usual habits, in the morning or middle of the day, so that it suddenly begins to burrow in the ground or to wall up a cranny. This is an example of a great class of problems presented by the existence of critical points in the life-history of animals. Why do birds in autumn feel the impulse to migrate, in spring to build nests; why does a caterpillar at a certain time begin to spin its cocoon, knowing nothing of the pupa stage upon which it is about to enter? And so on. Such changes seem to depend (a) on external conditions of temperature, food-supply, etc. ; $(b)$ on internal conditions, a certain maturing or modification of the organism, producing perhaps an uneasiness that is relieved by a certain action. In wasps the approach of the time for laying an egg brings on a complete change of behaviour, so that instead of sporting about amongst the flowers, paying no attention to insects or animals of any other species, she begins to burrow, or to catch bees or spiders. By merely natural-history methods we 


\section{The Neural Basis of Instinct}

cannot explain this : it is intrinsically a physiological question. But perhaps Psychology will help us to something better than mere blank astonishment. . . .

"If it seems difficult to develop such chain instincts as these wasps display by natural selection of occasional small variations, or even of considerable variations, such as the bringing of a second or third fly or bee, there has been plenty of time to do it in. Hymenoptera are found throughout the Tertiary strata, perhaps even in the middle of the Secondary (Jurassic) - a good many million years ago. Similar species of wasps and ants with similar habits are found in North America and in Europe, and must be supposed to have spread when the Arctic regions were viable; for so many resemblances can hardly be accounted for by such methods of migration as the occasional transportation of colonists by floating timber."

These excerpts give some idea of the nature of Read's analysis. He does not describe the nervous mechanism and processes by which these actions are determined. This would be a very difficult thing to do in so small an animal as the wasp, and it will probably be a long time before this can be done. But we know enough about the nervous system to have reason to believe that these chain instincts are being determined by it in the wasps as in other animals. Read recognizes this when he says that the explanation of these instincts is "intrinsically a physiological question." 


\section{CHAPTER XIII}

THE PRINCIPAL HUMAN INSTINCTS AND GENERAL INNATE TENDENCLES

The number of human instincts, 238. - The directing of instinctive tendencies by intelligence, 239. - Description of the principal human instincts, 240. - The classification of the instincts, 244. - The nature of a general innate tendency, 245. - Imitation, 246. - Suggestion, 247. - Sympathy, 248. - Play, 248. Emulation, 251. - Workmanship, 252. - Gregariousness, 252. Habit, 254 .

LET us now turn our attention to the human instincts. Many lists have been prepared which are supposed to include all or at least the most important of the human instincts. But there has been the greatest variation among these lists both as to what actions are to be included in the list as being instinctive and also as to the total number of human instincts. The last point is illustrated in the cases of Darwin and James. Darwin thought that man had fewer instincts than any other animal, while James thought that man had the largest number of instincts of any animal. It goes without saying that such a difference of opinion would arise primarily out of a difference of opinion as to what ought to be included under the head of instinct.

But there are other reasons back of this reason. Those who believe that man has very few instincts have usually been greatly impressed by the extent to which human actions are determined by intelligence. They therefore 


\section{The Principal Human Instincts}

assume that where intelligence has appeared, instinct has died out. Those who believe that man has numerous instincts may recognize fully the part now being played by intelligence. This, however, does not explain why man should have more instincts than other species, and I have seen no explicit attempt to explain this. I presume, however, that the explanation in the minds of most of those who have taken this position, though no one of them may have stated it, is the following: As we have seen, instinctive actions are determined by the nervous system. Consequently, the number and the complexity of the instincts would depend upon the extent and the complexity of the nervous system. It is therefore to be expected that the higher animals with their more extensive and complex nervous systems would have more instincts than the lower animals. This would be most true of man.

But it is also true that these instincts are very frequently not at all obvious in the behavior of men, thus giving rise to the idea that man has few instincts. It has been shown how an instinctive tendency is inhibited by becoming concentrated upon one object which thereafter serves as the only stimulus for it. Where instincts' are numerous they may be inhibited and therefore become apparently non-existent by neutralizing each other where they are contradictory. In all probability, however, the principal reason why instincts apparently disappear in the higher animals and especially in man is because the instinctive tendencies are being guided and influenced more and more by past experience and therefore by intelligence. Thus while the instincts are still at work furnishing the primary motive power, they become more or less hidden from view 
by these later mental phenomena. James has described this situation very well in the following words: "Wherever the mind is elevated enough to discriminate; wherever several distinct sensory elements must combine to discharge the reflex arc; wherever, instead of plumping into action instantly at the first rough intimation of what sort of a thing is there, the agent waits to see which one of its kind it is and what the circumstances are of its appearance; wherever different individuals and different circumstances can impel him in different ways, - wherever these are the conditions, we have a masking of the elementary constitution of the instinctive life." We have already seen that even in the lower species instinctive actions are not absolutely invariable and predictable. For the reasons stated above, these actions in the higher species become far more variable and far less predictable.

\section{The Principal Human Instincts}

Let us now consider some of the lists of human instincts which have been prepared. The first will be James' list of what he considers the principal human instincts. As might be expected of James, the list is quite extended. First he mentions a number of simple reactions which the psychologists call impulses and which many writers have regarded as being mere reflexes without being instinctive. Among them are the following acts which characterize infants very early in life: Crying, sneezing, snuffling, snoring, coughing, sighing, sobbing, gagging, vomiting, hiccuping, starting, moving the limbs when tickled, touched, or blown upon, etc. If any one of these reactions is a simple reflex, it does not fall under my definition of an 


\section{The Principal Human Instincts}

instinct. But if it is an "inherited combination of. reflexes," it is evident that it must fall under my definition, for every one of these actions is external. Then he mentions a number of more complicated actions which appear very early in the life of the infant and which are still more certainly instinctive in their character. Among these are the following: Sucking, biting, clasping, carrying to the mouth of an object, smiling, turning the head aside, as a gesture of rejection, holding head erect, sitting up, standing, locomotion, vocalization, etc.

We now come to very. complex forms of behavior, and it may be questioned whether some of them are distinct instincts. Among them James names the following: Imitation, emulation or rivalry, pugnacity, anger, resentment, sympathy, the hunting instinct, fear, appropriation or acquisitiveness, constructiveness, play, curiosity, sociability, shyness, secretiveness, cleanliness, modesty and shame, sexual love, jealousy, parental love.

It is very doubtful if imitation can be considered an instinct, since it does not involve any specific reflexes or mode of behavior. In the course of imitation any kind of act may be performed. As we shall see, imitation is caused by suggestion and is like instinct only in that the tendency to imitate is inborn. Much the same can be said of emulation or rivalry. It certainly does not involve any specific reflexes or mode of behavior. In the course of rivalry any kind of act may be performed. It is probable that it is to a certain extent imitation, but imitation which is stimulated in part at least by the instinct of pugnacity. I shall discuss it later as one of the general innate tendencies.

Pugnacity is in all probability an instinct, since it usually 
involves a well-defined mode of action. But anger and resentment are simply emotions which accompany pugnacious acts, and as such cannot be called instincts.

Sympathy is primarily a state of feeling. Various acts result from this state, but they are very diverse in character, and so far as sympathy implies external action, it is a general innate tendency.

The inborn tendency to hunt is in all probability an instinct, since it involves a well-defined mode of action.

Fear is an emotion rather than an instinct, but it usually accompanies an instinctive act, the most frequent one being flight.

Appropriation or acquisitiveness is probably an instinct. Constructiveness may possibly be an instinct, though it involves a good many different kinds of action.

The tendency to play is undoubtedly inborn, but it involves so many different kinds of action that it can hardly be regarded as a distinct instinct. It is rather a general innate tendency.

Curiosity may have an instinctive basis, but in man is in large part an intelligent phenomenon.

Sociability and shyness are undoubtedly inborn, but are so general that it is doubtful if they are distinct instincts.

Secretiveness and cleanliness are probably instincts, since they involve fairly definite modes of action.

Modesty and shame certainly are not instinctive, as James himself admits in his discussion of them.

Sexual love is, strictly speaking, an emotion. But the sexual instinct is one of the most powerful of the instincts. Jealousy is an emotion which is aroused when sexual love is violated or the sexual instinct is thwarted, and may ac- 


\section{The Principal Human Instincts}

company various instinctive acts usually pugnacious in their character.

Parental love is, strictly speaking, an emotion. But the parental instincts which cause parents to care for their young are very important instincts.

The difficulty involved in determining in the case of many of these modes of behavior whether or not they are instinctive is that we do not know what nervous mechanism is involved. All of the modes of behavior which have been discussed are probably inborn, with the exception of modesty and shame, but we cannot be sure in every case whether there is involved a sufficiently definite combination of reflexes to justify calling it an instinct. Where, however, we find a great variety of acts grouped under the head of one instinct, as in so many of the above cases, it is hard to believe that it is an instinct, but rather a general innate tendency which involves a good many different kinds of instinctive acts. These tendencies will be discussed more fully later in this chapter.

Angell ${ }^{1}$ says that the generally recognized instincts in man are the following: "Fear, anger, shyness, curiosity, affection, sexual love, jealousy and envy, rivalry, sociability, sympathy, modesty(?), play, imitation, constructiveness, secretiveness, and acquisitiveness." Angell says elsewhere ${ }^{2}$ in this book that instincts "represent structurally preformed pathways in the nervous system, and stand functionally for effective inherited coördinations made in response to environmental demands." In view of this statement it is strange that he should include as instincts such general tendencies as imitation and play, which do

1 James R. Angell, Psychology, p. 349.

Op. cit., p. 339. 
not represent any specific pathways or coördinations in the nervous system, but which manifest themselves through many reflexes and combinations of reflexes. I have already commented upon all in his list with the exception of affection and envy. Both of these are emotions which are frequently accompanied by instinctive actions.

McDougall ${ }^{1}$ gives the following list of what he considers the principal human instincts : Flight, repulsion, curiosity, pugnacity, self-abasement, self-assertion, parental, reproductive, gregarious, acquisitive, constructive. I have already commented upon most of these and will speak briefly of the others. Flight is undoubtedly an instinct and is usually the instinctive act which is in mind when fear is spoken of as an instinct. Repulsion is probably a distinct instinct. Self-abasement and self-assertion are probably innate tendencies, but it is hard to say whether they are sufficiently specific to be called instincts. McDougall associates very closely with each of these instincts an emotion which is aroused by it. The subject of emotion I shall discuss in a later chapter. He also discusses some of the general innate tendencies which will be taken up further on in this chapter.

Several writers have tried to classify the instincts according to the ends which they accomplish, as, for example, when they are classified according to whether they preserve the individual, the species, or society. It is true that some distinction may be made between instincts according to the above criterion. And yet the instincts are so closely bound up together that it is dangerous to make very much of such distinctions. After all, the in- 
stincts, like all other characteristics of organic matter, are, so far as they have any end, directed towards the general end of increasing the amount of living matter. So that the preservation of the individual, the species, and of society in the long run aid each other and accomplish the general end of increasing the amount of living matter. And frequently it is impossible to determine whether an instinct preserves one of these three any more than it does the other two.

Furthermore, the attempt to classify instincts according to such a criterion usually leads to the inclusion under the head of instinct of every mode of behavior which attains these ends, even though many of them are far too complex to be regarded as instincts. We have a good example of this in the case of the psychologist, Henry Rutgers Marshall, who in his work entitled Instinct and Reason classifies the instincts as follows: (I) instincts of individualistic import, (2) instincts relating to the persistence of organic species, (3) instincts relating to the persistence of social groups. Then under these heads he recognizes ethical, patriotic, benevolent, and artistic instincts, while most of his book is devoted to trying to prove that there is a religious instinct in man which has had biological and social value. As we have already seen, all of these "instincts" are very complex modes of behavior which are made up of instinctive, habitual, and intelligent actions which have become closely associated with each other.

\section{General Innate Tendencies}

Let us now turn to the general innate tendencies and discuss them briefly. As we have already seen, there are 


\section{The Science of Human Behavior}

certain general, inborn modes of behavior which are regarded by many as distinct instincts, but which cannot rightfully be regarded as such, because at different times they involve totally different parts of the nervous system. Inasmuch as an instinct has a specific neural mechanism which is utilized every time the instinctive action takes place, a mode of behavior which involves at different times entirely different parts of the nervous system cannot be regarded as an instinct.

As we have seen, imitation cannot be regarded as an instinct, because it involves very different modes of behavior. Furthermore, there are certain kinds of imitation which are not caused by an inborn tendency. Rational imitation which results from experience and intelligence will be excluded from the present discussion. Taking, then, the imitation which results from an inborn tendency, let us see what is its nature. It is evident that imitation cannot take place until the act in question has first been performed by another. It is then performed by the imitator, because he has seen the act performed or has in some other way become aware of its performance. The act may be an instinctive one and therefore one which is usually aroused by certain appropriate stimuli which are directly connected with the neural mechanism of the instinct. But when the act is imitated, it is not aroused by external stimuli which are directly connected with the neural mechanism of the instinct. The centers in the nervous system which are stimulated by these external stimuli must therefore be connected within the central system with the motor centers for the instinctive act. It is therefore evident that imitation is ideo-motor action. It is an 


\section{The Principal Human Instincts}

innate tendency in the sense that the association centers in the brain make it possible for the motor centers of this mode of action to be aroused from other centers which have been stimulated by external stimuli coming from the performance of this act by another. These stimuli may be visual, auditory, olfactory, tactile, etc., in their character, but whatever they may be they are connected with the motor centers for certain modes of action and may prove effective thus indirectly to stimulate these modes of action.

This process by which a stimulus is transmitted from a receptor center by means of an association center to a motor center is suggestion, and the suggestibility of an individual depends upon the ease with which such transmission takes place within his central nervous system. I am well aware that suggestion is frequently defined otherwise, as when the communication of an idea from one individual to another is called suggestion. But I believe that my definition is the only correct technical one for this process. It is usually assumed that suggestion leads to action of the sort suggested more or less directly and, if this be the case, it must be by means of such a process as has been defined above as suggestion. It is true that communication of an idea may lead to action, but the connection in that case is much more indirect and the process much more complicated. Suggestion is sometimes called a general innate tendency. But if it is meant to imply by this that it is an external form of behavior, it certainly is a mistaken definition. For, as we have seen, suggestion is a purely internal process, but leads to an external mode of behavior in the form of imitation. 
Sympathy also, like suggestion, is an internal process which leads to the stimulation in one person of an emotion already experienced by another. Like suggestion it also has been mistakenly called a general innate tendency in the sense of being an external mode of action. But socalled sympathetic action is nothing more than imitative action which is accompanied by sympathetically induced emotion.

Playful activities are very widespread among living beings. Play may be defined as the expenditure of energy purely for the sake of gaining pleasure without being directed towards any useful purpose. At any rate, no useful purpose can be the immediate object of play, though, as we shall see, it may serve some useful purpose in the long run. Playful activities are most frequent among the young, who very often spend all their time in play. The simplest theory of play is the Schiller-Spencer surplus energy theory of play. According to this theory, animals play in order to get rid of what energy they have left over after they have completed the activities necessary for existence. There is undoubtedly a great deal of truth in this theory. It is a well-known physiological fact that an organism cannot remain healthy unless it performs its functions to a normal degree. In order to perform these functions it has to expend a certain amount of energy. It is stimulated in the first place to perform these functions by the necessities of existence. If these necessities require it to expend the normal amount of energy, it will become normally tired and will not need to expend any more energy.

The surplus energy theory of play therefore explains in a 


\section{The Principal Human Instincts}

general way why animals expend energy in ways which are, to say the least, not immediately useful. But this theory does not explain the forms these activities take. If play is nothing more than the expenditure of surplus energy, it might be expected that it would be nothing more than formless activity. But, as a matter of fact, playful activities take on very definite forms, and these forms are suggestive of their nature and origin. The noticeable thing about them is that they resemble certain instinctive activities. Thus the plays of the boy seem to reveal the presence in him of the hunting and combative or pugnacious instincts, while the little girl playing with her dolls reveals an early awakening of the maternal instinct. It is true that imitation has a good deal to do with determining the playful activities of the young. Very frequently these activities are nothing more than imitations of the activities of their elders. But when such playful activities are quite spontaneous, that is to say, do not copy in any way the activities of the adults among whom these young live, it is usually possible to detect strong likenesses to instinctive activities. A good way to test this is to separate entirely from the earliest age the young of a species from adults of the same species and then observe the playful activities which spontaneously manifest themselves among these young. For example, if a number of very young puppies are isolated from other dogs, it is certain that as soon as their eyes are open and their legs are strong enough for them to move around easily, they will begin to fight each other in play, thus revealing an early awakening of the combative instinct. But the instinctive impulse will not display itself in its full strength, for they will not usually 


\section{The Science of Human Behavior}

hurt each other, but will simply go through the motions of fighting. Apparently, therefore, the inborn, integrated series of reflexes upon which the combative instinct is based is already beginning to function, though not yet with the facility and force it will display as maturity is reached. Thus it appears, as has been well shown by Groos, ${ }^{1}$ that playful activities take the form, to a large extent, of early manifestations of instinctive impulses which will attain full force as maturity is reached. And as has been pointed out by Groos and others, such playful activities have great utility for the young after they have grown up and for the species, for in these playful activities skill is acquired in the practice of the instinctive activities which will have great utility later in life. Thus we see why it is that though playful activities have no immediate utility, they may have great utility for the future.

It is also true of the playful activities of adults that they are to a large extent the manifestation and expression of instinctive impulses. Thus in most games are involved opposition and conflict, which are undoubtedly manifestations of the combative instinct. Indeed, it has become true in our modern civilized society that some instinctive impulses now find their expression for most individuals only in the form of play. For example, few people to-day need to hunt for purposes of securing subsistence, which was undoubtedly the original cause for the growth of the hunting instinct, but many indulge in hunting for purposes of play. While the utility of playful activities, the forms of which are determined by instinctive impulses, has been shown for the

1 Karl Groos, The Play of Animals, translated from the German, New York, 1898. The Play of Man, translated from the German, New York, Igor. 
young, it is perhaps unfortunate that the same should be true to so great an extent for adults, for if playful activities differed greatly. from purely instinctive activities, there would be greater variety in human behavior, and human personality would be correspondingly more complex.

It must now be evident that very different kinds of activities are included under the head of play. If, therefore, an instinct is what I have defined it, namely, an integrated series of reflexes, play certainly is not a specific instinct, as some writers have contended. The inborn physiological characteristics require that surplus energy be expended, and the instincts furnish well-worn grooves into which the expenditure of this energy may fall, so that playful activities frequently are also instinctive activities, but taken together they do not constitute a distinct instinct.

Emulation or rivalry, which, as we have seen, is regarded by some writers as a specific instinct, is much the same kind of a mental phenomenon as imitation or play. As has been stated, it does not involve any one integrated series of reflexes, and in the course of emulation an individual may use many different kinds of behavior. As a matter of fact, emulation is to a large extent imitation stimulated in part at least by the combative or pugnacious instinct. Other instincts are involved according to the object of emulation or rivalry. For example, if it is rivalry in love, the sexual instinct plays a part. If it is rivalry in economic activities, the instinct of acquisition plays a part. Thus under the head of emulation we place certain complex groups of activities which as groups bear certain likenesses to each other. These groups include many activities which are instinctive. 


\section{The Science of Human Behavior}

Another so-called instinct is the "instinct of workmanship." 1 It is evident that the tendency to work involves many different kinds of activity. Work may be defined as being effort devoted to the production of things of value. Like play, it is undoubtedly due in part to the physiological need of the organism to expend a certain amount of energy. But it is also and in large part due to the needs of subsistence which force the individual to expend effort in order to secure the things needed. In human society this takes the form of economic pressure in the economic struggle for existence. This effort is directed and its character is determined in part by numerous internal factors. Among them are instincts such as the instinct of constructiveness, which has been discussed, and various feelings and emotions which will be discussed in later chapters. Furthermore, intellectual factors play a more and more important part, especially among men. Various compounds of instincts, intellectual characteristics, and feelings arise which plawa part in directing the tendency to work. Among them may be mentioned æsthetic characteristics, such as the so-called architectonic sense, ambition to secure wealth, power, etc., certain altruistic sentiments, etc. This effort is also directed and its character determined in part by numerous factors of the physical and social environment of the individual. So that it must be evident that this so-called "instinct of workmanship" is very complex in its character and causes, and very far from being a distinct instinct.

Many writers recognize a distinct instinct of sociability or gregariousness. It is true that many of the higher species

1 Thorstein Veblen, The Instinct of Workmanship and the Irksomeness of Labor, in the Am. Jour. of Sociology, Vol. IV, No. 2 (Sept. 1898), pp. 187-201; The Theory of the Leisure Class, New York, 1899. 
are gregarious, so that the members of these species live in more or less constant association with each other. Furthermore, frequently there does not seem to be any other reason for such association than an innate impulse causing these sociable animals to come together. This is probably why so many have thought that there must be a gregarious instinct. But when we apply our definition of an instinct as an integrated series of reflexes, it is hard to believe that there is any such specific series of reflexes which causes association. However, it is certain that the primary causes of association were instincts so far as association came about through subjective causes, while ever since they have been powerful forces for association. The principal ones of these instincts undoubtedly are the sexual and the parental instincts. But habit also has a great deal to do with gregariousness. The young of all the higher species are helpless for a certain period of time and have to be cared for by theic parents. During this time the habit of association becomes deeply rooted. Furthermore, during this period imitation leads them to act like their fellows. Thus it is that these habits mightily reënforce the instincts which lead to gregariousness. We should also not ignore the external forces which drive members of the same species together. Frequently the food and drink of a species are to be found only at certain places, so that the members of the species are forced to gather at those places, as, for example, at the salt licks where the American bison used to gather. Or the members of a species are forced to come to certain places for shelter or protection. Natural selection is at work determining the extent to which a species is to become gregarious. As a general thing it is the weaker species, 
which are therefore preyed upon, which become the most gregarious, for the less sociable members who wander from the protection of the flock or herd are pretty certain to be destroyed. The strongest species, like the lion, the tiger, the hawk, etc., which have little to fear from other species and which spend their time in preying upon the weaker species, are usually very unsocial.

In human social evolution many conscious and rational forces are at work bringing about association in the form of mutual aid and coöperation and, above all, in the division of labor. But I cannot stop to discuss these causes of association in this chapter. Enough has been said, I think, to show that the forces which bring about association are very complex and that it is very doubtful if there is any specific instinct of gregariousness. In later chapters on social evolution the causes of association will be analyzed more fully.

So far as I know, no one has called the tendency to form habits an instinct, but it has been called a general innate tendency, and its relation to instinct should be briefly noted. It is undoubtedly the tendency of all living organisms to perform an action more easily on repetition. After this ease has been gained, the act is spoken of as a habit. But the question may be raised as to what kind of an act it was before the habit was formed. It is evident that it must have been some form of inborn reflex action and may have been instinctive. A habit may therefore grow directly out of an instinct, so that the connection betweeen instinct and habit is very close. A habit may be an instinctive mode of action which has become reënforced through repetition, or it may be a combination of instinctive modes of 


\section{The Principal Human Instincts}

action. The tendency to form habits is inborn, while the modes of action which are to become habitual are at first inborn, though they may vary in the course of habitual use. Lloyd Morgan has called these habits which grow out of instincts "instinct-habits," which phrase emphasizes the close connection between instinct and habit.

Habits are sometimes spoken of as reflex actions. According to the strict definition of reflex action which I have been using, this is not accurate, for reflexes are inborn, while habits are acquired. The reason for speaking of habits as if they were reflexes is that habitual acts are frequently performed in the same more or less invariable and unconscious fashion as reflex acts. It is true that habits are formed by reënforcing or combining reflex and instinctive actions, so that habits are always based upon reflexes, but as habits are not mere reflexes.

The discussion of instinct in these chapters must, I think, have shown that our knowledge of instinct will depend in the main upon our knowledge of the nervous system. It is impossible to define instinct in general, to determine what are the specific instincts, or to describe the mechanism of these instinctive actions apart from the nervous system. There is, probably, no more important line of investigation for the understanding of the behavior of the higher animals than the study of the nervous system. 


\section{CHAPTER XIV}

\section{THE NATURE OF INTELLIGENCE}

Psycho-physical processes, 256. - Introspection, 257. - Intelligent behavior as the result of experience, 258. - Associative memory as a criterion of intelligence, 260. - Unspecialized nervous tissue essential for intelligence, 262. - The cerebral cortex as the neural basis for intelligence, 263. - The central nervous system as the organ of memory, 265. - The range of intelligence in the animal world, 267 . - The structural advantages of the vertebrate for the development of intelligence, 269. - The reasons for man's superior intelligence, 270. - The nature of learning, 273. - Sensations, 274. - Images, 274. - Memory, 275. - Sensori-motor and ideo-motor action, 275. - Pleasure and pain as reënforcing and inhibiting ideo-motor action, 275. - Ideas, 277. - Thought as a flow of ideas, 277. - Concepts as generalized images, 278. - Imageless thought, 278. - Reason, 279. - The nature of human intelligence, 280 .

I HAVE now discussed the three important forms of behavior, the tropism, the reflex action, and the instinctive action. Each of these is external; that is to say, it is a movement of an animal or part of an animal which is visible from the outside. It may be that we are not justified in calling any movement of the organism which is not visible from the outside a form of behavior. But there are certain internal movements which are physiological processes like these forms of behavior, and which have great influence over certain kinds of behavior. These internal processes, consisting of numerous minute and refined movements, most of which are within the nervous system, are usually 
called psycho-physical processes, and collectively they determine what are called mental phenomena. Because of the important part played by these processes in the determination of the behavior of the higher animals, we must now consider them. They will be discussed under the heads of intelligence and consciousness, while at the end of this discussion I shall state my conception of their fundamental nature in the form of a theory of mind.

The study of mental phenomena has always presented great difficulties. Because the movements involved are internal and are very minute and refined in their character, they are more or less intangible and difficult to observe. So far the method used in studying mental phenomena has been subjective and introspective rather than objective. Introspection must always be an important source of information with regard to mental phenomena, but I do not believe that the study of mental phenomena can become thoroughly scientific until the method used is in the main objective. The present study will therefore be from the standpoint of behavior and will consequently be objective, though occasional recourse to introspection will be made.

\section{NATURE OF INTELLIGeNT BeHAvior}

The phrase "intelligent behavior" is frequently used, the use of which may seem to imply that intelligence is a form of behavior, or, at any rate, that it is an organ which determines that form of behavior. As a matter of fact, however, it is not a distinct organ, but stands in the same relation to intelligent behavior that instinct stands to instinctive action. Let us see what are the peculiar features of intelligent behavior. Such behavior always grows out of experience. 
No organism without individual experience can display intelligent behavior. Intelligence is not inherited, but is acquired by the individual. Intelligent behavior is not an inherited form of reaction, though it is based upon and makes use of inherited modes of behavior. It is, in fact, a modification of an inherited mode of behavior due to experience and is consequently more varied usually than inherited modes of behavior, though, as we have seen, inherited modes of behavior also may vary somewhat.

Intelligent behavior is therefore made up of tropic, reflex, and instinctive actions which have been combined in new ways as a result of experience so as to constitute new forms of behavior. It is evident, therefore, that the organism must be amenable to the effects of its experience in order to develop these new forms of behavior. In other words, the organism must possess a certain degree of plasticity, and in this plasticity we find the necessary structural basis for intelligent behavior. Now it goes without saying that all organisms possess a certain degree of plasticity. This is due in the first place to the fact that all matter is affected by the forces which play upon it. But in the second place the protoplasm of which all organisms are constituted is peculiarly sensitive to external forces. So that all organisms are more or less plastic, and the behavior of every organism is being affected by its experience, as has been shown in the preceding study of inherited forms of behavior. But the degree of variation in behavior which is sufficiently great to justify calling it intelligent requires an unusually high degree of plasticity. It requires parts of the organism which are unusually sensitive to the effects of experience and which are specially devoted to combining and rearranging the inherited modes of 
behavior. The principal example of this is the unspecialized correlation tissue or gray matter of the nervous system. In fact, we may find that no intelligent behavior is possible without the presence in the organism of this gray matter.

In the light of the preceding remarks it is evident that it is important to determine where the line is to be drawn between the plasticity in behavior which is common to all organisms and the plasticity which may be called intelligent. It is true that some writers have refused to draw any such line and have insisted that all organisms are capable of intelligent behavior. For example, some of the students of the behavior of the lower organisms, who have naturally been impressed with the continuity in the development of behavior, attribute intelligence even to the lowest organisms when their behavior varies in accordance with experience. This is true of Jennings, who, as we have seen, always tends to minimize the differences between the behavior of the lower and that of the higher organisms: He endeavors to show that perception, discrimination, choice, attention, pain, fear, memory, habit, and consciousness are to be found in a rudimentary form even in the protozoa. ${ }^{1}$ As we have seen, the work done by Jennings and his fellowworkers has been of the greatest value in tracing the evolution of behavior and in emphasizing its continuity." But if intelligence is extended to all organisms, the term will lose its value for distinguishing between different kinds of behavior, just as we have seen the term instinct would lose its utility if it was reduced to reflex action, and the term reflex would lose its utility if it was reduced to tropism. It is therefore better, I believe, to restrict the term intelli-

1 Behavior of the Lower Organisms, New York, 1906, pp. 329-337. 
gent to certain of the more complex variations in behavior and to formulate a criterion for distinguishing between intelligent and unintelligent behavior.

\section{Associative Memory}

What, then, is to be this criterion? Various attempts have been made to formulate such a criterion. Perhaps the best-known one is the theory that associative memory or the power of forming associations is the mark of intelligence. One of the principal exponents of this theory is Loeb, who defines associative memory as follows: "By associative memory I mean that mechanism by which a stimulus brings about not only the effects which its nature and the specific structure of the irritable organ call for, but by which it brings about also the effects of other stimuli which formerly acted upon the organism almost or quite simultaneously with the stimulus in question. If an animal can be trained, if it can learn, it possesses associative memory." 1 Again he characterizes it as follows: "By associative memory I mean the two following peculiarities of the central nervous system: First, that processes which occur there leave an impression or trace by which they can be reproduced even under different circumstances than those under which they originated. . . . The second peculiarity is, that two processes which occur simultaneously or in quick succession will leave traces which fuse together, so that if later one of the processes is repeated, the other will necessarily be repeated also." 2 Loeb speaks of associative memory primarily as the criterion of consciousness and of psychical phenomena

1 Comparative Physiology of the Brain and Comparative Psychology, New York, 1900, p. 12. 


\section{The Nature of Intelligence}

in general, but it is evident that he is including intelligence under this head.

Various other writers have adopted this theory of the criterion of intelligence, among them being Holmes, ${ }^{1}$ Lloyd Morgan, Forel, Bethe, etc. But there have also been severe critics of this theory, among them being Yerkes, ${ }^{2}$ Claparède ${ }^{3}$ and Jennings. ${ }^{4}$ The essence of their criticism is that associative memory simply means the ability to learn, and that this ability is possessed by all organisms. It is evident that by the ability to learn they mean merely the capacity to be modified, which, as we have seen, is possessed by all protoplasmic matter. If the ability to learn simply means this modifiability, and if associative memory is nothing more than this ability, it is evident that this criterion is of no value, for in that case associative memory and all that its presence implies would characterize the whole organic world. Let us see whether such is the case.

It is true that Loeb says that if an animal can learn, it possesses associative memory. But it may be that he has a special kind of ability to learn in mind, and an analysis of his definition shows that he has. The kind of learning he has in mind is indicated when he says that associative memory is present when a stimulus arouses not only the response which is peculiar to it, but also a response which is peculiar to a stimulus which has previously acted upon the organism. It is evident that this cannot happen unless the animal has a mechanism which keeps a record of the move-

IS. J. Holmes, The Beginnings of Intelligence, in Science, March 31, I91r.

2 Animal Psychology and Criteria of the Psychic, in the Jour. of Phil. Psych. and Sci. Meth., Vol. II, No. 6 (March r6, r905).

3 E. Claparède, The Consciousness of Animals, in the International Quarterly, Vol. VIII (Dec.-March 1903-1904).

Op. cit., p. 334 . 
ment stimulated by the previous stimulus so that when it is stimulated by the second stimulus it can reproduce the previous movement. It is possible that an organism without a nervous system could do this to a very slight extent, but its ability along this line certainly would be very limited, for the effect of the movement would probably be diffused more or less all over the organism, since there would be no part specialized for the purpose of keeping a record. It is evident from the citations made above that Loeb regards the associative memory as a peculiarity of the central nervous system, so that the learning it involves must be of a special kind and not merely the plasticity and ability to modify which characterizes all organic matter.

\section{NeUral Basis of Intelligence}

I believe, therefore, that we are safe in drawing the line between intelligent and unintelligent behavior, between conscious and unconscious behavior, between psychic or mental and non-psychic behavior at the point where we pass from the animals without a central nervous system to those with a central nervous system. As we shall see, it may be possible to draw this line still higher up in the evolutionary scale. By this I mean that an animal without a central nervous system is incapable of intelligent and conscious behavior and cannot manifest psychic phenomena, but that such behavior and phenomena may appear as the central nervous system develops. Unless we limit the range of intelligence and consciousness, the terms will be meaningless. The reasons for drawing the line at the point named will appear as we trace briefly the evolution of intelligence and consciousness. 
In preceding chapters has been shown briefly the part played by the nervous system in the behavior of the higher animals. It has been shown that, broadly speaking, the function of the nervous system is to integrate the movements of the organism. This is accomplished by the simple and the compound reflexes which are inherited. When a combination of these reflexes causes an external movement, it constitutes an instinct. These reflex and instinctive actions are usually adaptive, for natural selection has been at work preserving those individuals which display useful inherited reactions. Such adaptation is therefore racial. But as we go higher in the animal scale we find usually more and more capacity for individual adaptation, and some of these adaptations constitute intelligent behavior.

The inherited reactions are more or less fixed at birth, so that the parts of the nervous system which determine these reactions are already specialized for these purposes at birth. In order, therefore, that it be possible for adaptations to take place, there must be parts of the nervous system which are not specialized at birth. We find the principal example of such unspecialized nerve tissue in the cerebral cortex or gray matter of the brain. ' It is therefore reasonable to suppose that intelligence will vary in accordance with the amount of gray matter. As we have seen, such is the case, and intelligence is very directly proportioned to the amount of gray matter in proportion to the total size of the body. The amount of the gray matter is, as we have seen, determined in the main by the size of the cerebrum and by the number of convolutions in the cerebral cortex. In this unspecialized nerve tissue, association paths become established which correlate the inherited modes of 
action into new combinations which constitute new forms of behavior which we may call intelligent. This is what leads Herrick to speak of the intelligence as the function of the cerebral cortex. "In the broad view we may say that intelligence is a function of the cerebral cortex, but only in the sense that here are found the most complex correlations in the chain of vital response whose initial phase is to be sought in the environment which supplies the stimulus and whose final phase is also found in the changes wrought in the environment by the bodily reaction." 1

Let us then discuss briefly the evolution of intelligence. The preceding chapters have shown that every animal starts out with an equipment of inherited reactions. The forces of the environment are constantly at work upon the animal, causing these reactions to vary somewhat, reënforcing some of them, inhibiting others of them. But these variations are not necessarily intelligent. The cumulative effect of some of these forces may cause changes in. the structure of the animal which will cause permanent changes in its behavior. These permanent changes are rudimentary forms of habit and of learning. I have described instances of these in the chapters on the behavior of the lower animals. But these lower animals do not possess, so to speak, a special mechanism for recording the effects of past stimuli, so that these effects may be more or less faithfully reproduced in the future by new stimuli which may be similar or entirely different from the original stimuli. Such a mechanism appears in the form of the central nervous system so that as soon as this system begins

${ }^{1}$ C. J. Herrick, The Evolution of Intelligence and its Organs, in Science, Vol. XXXI, No. 784 (Jan. 7 , 1910). 
to develop, intelligent behavior may become possible. Let us see why this is the case.

The central nervous system is specially adapted for recording the effects of stimuli. The association areas of the cerebrum act as the special organ of memory. It goes without saying that a rudimentary form of memory exists without this organ because of the plasticity of all organic matter, which we have already noted. But this organ retains an unusually clear-cut and well-defined record of these effects. But if this was all that this organ accomplished, intelligence would not be possible, for these effects would be reproduced only under the same circumstances as. when they were first produced. Within the central nervous system these effects are connected with other sense organs than the ones from which they first originated and with other motor organs than the ones through which they were originally discharged. This is why the kind of memory which is based upon the central nervous system is spoken of as associative memory. These memories do not stand alone by themselves, but become intimately interrelated in a very complex fashion. So it is that in an animal with a well-developed central nervous system which has acquired a large and varied store of memories, the behavior which results from a certain stimulus may be vastly different from the purely inherited reaction which would respond to that stimulus if these memories were not present to vary and complicate the behavior. Such behavior is intelligent, and the capacity for such variations in behavior constitutes intelligence.

A central nervous system is then a sine qua non for intelligence. But intelligent behavior will not appear necessarily as soon as the central nervous system. In fact, this 
organ must develop considerably before such behavior is possible. Furthermore, the inherited modes of reaction probably must be relatively modifiable before intelligence can make its appearance. It is doubtful if simple reflexes or compound reflexes which cannot be called instincts can furnish the basis for intelligent behavior. A sufficient number of instincts which are readily modifiable is necessary before there can be variations in behavior, which can be called intelligent. This fact alone indicates that intelligence is not possible until the central nervous system has developed considerably. According to the definition which I formulated in an earlier chapter, an instinct is an integration and correlation of reflexes by the central nervous system. This kind of integration and correlation is inherited and is performed by the sensory and motor parts of the central nervous system, which are specialized for these purposes. When a certain number of these instincts which are relatively modifiable have evolved, and when the central nervous system has developed parts which are not specialized at birth, so that they can serve as association areas, then intelligence may make its appearance. ${ }^{1}$

I In view of the present-day vogue of the Bergsonian philosophy I feel it necessary to call attention to the ideas of this philosopher with regard to the relation between instinct and intelligence. According to Bergson the ability of the intellect to acquire knowledge is very limited, and with respect to life and living things in particular instinct in a sublimated form which he calls "intuition" is much more capable of acquiring knowledge. In view of our previous discussion of instinct and intelligence it is manifestly absurd to speak of the acquiring of knowledge by instinct. It is true enough that the capacity of the intellect to learn is very limited, but unfortunately it is the only means of acquiring knowledge. It is also true that from instinctive actions and from feelings, which Bergson confuses with instinct by identifying it with sympathy, the intellect learns a great deal. It is true enough that the intellect is unable to comprehend life as a whole with one all-seeing glance and bas to look at it piece by piece, so to speak, but mankind will not come any nearer to attaining this end by means of the mythical Bergsonian "intuition."

It is unfortunate that Monsieur Bergson, who is so well acquainted with modern 
Many experiments have been made upon many kinds of animals to determine whether they displayed intelligence. Unfortunately I have not the space to summarize these experiments here, interesting though it would be to do so. In many of these experiments it has been impossible to determine whether variations which were observed in behavior were intelligent or not. It may well be that it will never be possible to determine exactly at what point along the different lines of organic evolution the transition took place from unintelligent to intelligent behavior. This is to be expected in view of the continuity in the evolution of behavior. Because of this continuity, the transition would probably be gradual usually, and would usually if not always be imperceptible. It is nevertheless useful to define intelligence as we have been doing in order to distinguish broadly between the different types of behavior, even though we may not be able to tell in every case whether or not it is present. Furthermore, it is possible to tell in the great majority of species whether or not intelligent behavior is manifested.

\section{Distribution OF INTELLIGENCE}

It is probable that intelligence has appeared along several divergent lines of evolution whenever the requisite con-

biology and has so brilliantly restated the ancient conception of evolution as a process flowing without a break, should attempt to exalt this " intuition" over intellect, however weak iatellect may be. If we take Bergson's statement literally, this "intuition" is made up of instinct and sympathy and therefore consists of tendencies to acting and feeling, but not to thinking and knowing. However, it is probable that the phenomena which he has in mind and vaguely describes under the name of "intuition" in reality constitute an intellectual process and should be recognized as such. But like every intellectual process it derives much of its data from instinctive actions and states of feeling. I have not the space here to describe what this process is. 


\section{The Science of Human Behavior}

ditions described above have been fulfilled. Loeb says that it "can be shown that Infusoria, Colenterates, and worms do not possess a trace of associative memory," ${ }^{1}$ and therefore no intelligence. ${ }^{2} \mathrm{He}$ goes on to say that certain insects, such as wasps, have been shown to display intelligence. Experiments made by Yerkes on the crayfish, and other similar experiments, have indicated that crustaceans may possibly display faint glimmerings of intelligence, though it is rather doubtful. Numerous experiments have been made upon insects, which seem to indicate that intelligence appears occasionally among these animals. Among these experiments might be mentioned those of Forel, Wasmann, and Wheeler on ants, those of Lubbock and the Peckhams on wasps, and those of Lubbock and others on bees. It may be, however, that the presence of intelligence among the invertebrates is not certain. But when we turn to the vertebrates, we find certain evidence of its presence in all the higher vertebrates, while it may also exist to a slight extent among the lower vertebrates. Thorndike seems to have found evidence of it in fishes and Yerkes in reptiles. There is much more evidence of its presence in birds, while the evidence of its presence in all the mammals is probably complete. ${ }^{3}$

As has been said before, the transition from unintelligent to intelligent behavior has probably taken place

1 Op. cil., p. 13 .

2 "Le pouvoir associatif a subi un premier perfectionnement chez les crustacés et les insectes, grâce au perfectionnement des organes des sens, et surtout de l'œil, c'est-à-dire des appareils recepteurs; il a subi un second perfectionnement chez les vertébrés, grâce au développement des centres nerveux, c'est-à-dire des appareils enregistreurs." (G. Bohn, La naissance de l'intelligence, Paris, 1910, p. 342.)

'Since this chapter was written S. J. Holmes' The Evolution of Animal Intelligence (New York, 19ז I) has been published, which contains numerous illustrations of the presence of intelligence in different parts of the animal world. 
several, perhaps many, times along divergent lines of evolution whenever the requisite conditions were fulfilled. But the structural basis or action-system has been such in most of these cases as to prevent great development of intelligent behavior. This has been true of the insects. In them inherited modes of action in the form of reflexes and instincts have been carried to a high pitch of perfection. But in them, as in all the invertebrates, the action-system has apparently made possible only to a very slight extent, if at all, the individual modifications which constitute intelligent behavior.

But the structure, and therefore the action-system, of the vertebrates has been such as to make possible an extended development of these individual modifications in behavior. These characteristics of the vertebrates have been described, so that I need to refer to them only briefly here. The skeleton or bony structure which gives the necessary rigidity to the organism is in the case of the vertebrate on the inside, while in the invertebrate it is on the outside. This gives, generally speaking, more freedom of movement to the vertebrate, which can bend more easily and place itself in a greater variety of positions than the invertebrate. Furthermore, most of the exterior of the vertebrate is covered with sensitive tissue which enables it to receive many more impressions than the invertebrate, much of whose exterior is covered with a hard, insensitive tissue.

The vertebrate is arranged upon the general plan of a series of segments which are characterized by bilateral symmetry. In an organism so arranged the anterior segments, which habitually go first when the animal is in movement, receive many more impressions than the other 
segments. Thus the head and brain gain the enormous development which characterizes the vertebrate head and brain, and gives them their ascendancy in directing the behavior of the animal. The cerebrum, which, as we have seen, is the organ of intelligence, probably received most of its development from the impressions received through the sense organs in the head. "We owe to' the genius of Edinger the suggestion that the earliest stages in the origin of the peculiarities of the cerebral hemispheres must be sought in a study of the character of the reflexes connected with the nose and lips, particularly the feeding reactions. These have been termed collectively the 'oral sense' (Edinger) or 'Schnüffelsinn' (Kappers) and may perhaps best be called the muzzle refiexes." 1

\section{HuMaA INTELLigence}

These reasons for the development of the cerebrum have been peculiarly true in the case of man and the pre-human ancestors of man, and in this fact we can probably find the explanation for man's superior intellectual development. But not only the nose and lips have played a part in this development, but the arms and hands as well. Let us see how this development in all probability took place. I have already shown that intellect results from the associations established in the association areas of the cerebral cortex. Flechsig has shown that these areas constitute fully two thirds of the cerebral cortex in the human brain, a greater proportion than in any other animal. The problem is how man acquired so much association area. In the first place, in order to have more association area, the ${ }^{1}$ C. J. Herrick, op. cit., p. I4. 


\section{The Nature of Intelligence}

bulk of the cerebrum must be greater, and this means a larger cranium. Whether it was the pressure of a growing brain which forced the cranium to expand, or some other force, it is hard to determine, but it is quite likely that owing to the sutures which knit together the cranial bones, the cranium was able and did respond to the pressure of a growing brain. At any rate, since the primate type of mammal began to evolve, the cranium has expanded greatly, thus leaving room for the larger brain.

Flechsig, Johnston, and others have shown that the higher association centers are on the frontal and parietal convolutions of the cerebral cortex. Cunningham has summed up his theory as to the causes for the great expansion of these cortical areas in man in the following words: "I do not think that it is difficult to account for this important expansion of the cerebral surface. In the fore part of the region involved are placed the groups of motor centers which control the muscular movements of the more important parts of the body. These occupy a broad strip of the surface which stretches across the whole depth of the district concerned. Within this are the centers for the arm and hand, for the face, the mouth, and the throat, and likewise, to some extent, the center for speech. In man certain of these have undoubtedly undergone marked expansion. The skilled movements of the hands, as shown in the use of tools, in writing, and so on, have not been acquired without an increase in the brain mechanism by which - these are guided. So important, indeed, is the part played by the human hand as an agent of the mind; and so periectly is it adjusted with reference to this office, that there are many who think that the first great start which man ob- 


\section{The Science of Human Behavior}

tained on the path which has led to his higher development was given by the setting of the upper limb free from the duty of acting as an organ of support and locomotion.... In the same region of cerebral cortex, but at a lower level, there are also situated the centers which are responsible for facial expression." 1

Presumably, then, it was great stimulation of these motor centers for the arms, hands, face, and mouth which also stimulated the growth of the contiguous association areas. The next question would then be as to what caused this large amount of stimulation of these motor centers. It is of course impossible to reconstruct entirely the history of man's ancestors, but we can make some plausible conjectures as to its course. We have good reason to believe that the early primates were arboreal in their habits, as indeed is still true of most of the primates. It was probably the development of fruit on the late mesozoic or early tertiary trees which led to the habit of climbing. ${ }^{2}$ This habit naturally stimulated greatly the motor centers for the hands and arms. Just why some of the primates later on abandoned the trees and adopted terrestrial habits, now walking in a partially erect attitude, it is not easy to explain. But the fact that this change took place is certain, and some of the results from it can easily be conjectured. With its hands off the ground they could be used for finer adaptations than ever before, such as hurling missiles and later for using tools. On the ground it would be exposed to more dangers than in the trees. Vision and hearing would be used more than ever, and would consequently be greatly

1 D. J. Cunningham, in Nature, Sept. 26, rgor.

2 Cf. Joseph McCabe, The Evolution of Mind, London, I9ro, p. 257. 
strengthened and would furnish much material for the intelligence. In the face of dangers which these primates were not able to overcome by force because of the superior strength of many of the animals which preyed upon them, the more intelligent, which would therefore be the more wary and cunning, would be preserved, so that selection would be at work favoring the development of intelligence. In order to indicate all the factors which played a part in the development of man's superior intelligence, it would be necessary to review the whole course of man's early physical and mental evolution, which there is not the space to do here. Enough suggestions have been made to indicate how it may have taken place. It goes without saying that all that has been said so far has to do only with the evolution of the cerebral basis for intelligence. Much of the contents of the mind is passed on from generation to generation by education and tradition, so that it is not hereditary. But in order that this knowledge may be acquired and then transmitted from generation to generation, a certain cerebral basis is necessary.

\section{NATURE OF LEARNING}

I have now discussed briefly the nature of intelligence, the extent to which it prevails in the animal world, and the causes of the superiority of human intelligence. I shall now discuss a little further the nature of learning and the higher forms of intelligence. This will lead naturally to the discussion of consciousness, since it is impossible to discuss these higher forms apart from consciousness.

It has been shown earlier in this chapter that in one sense all organisms are capable of learning. That is to 


\section{The Science of Human Behavior}

say, because of the plasticity of organic matter the behavior of all organisms may be modified by the action of external forces. But it is rather absurd to call this learning. For example, to say that a plant learns is decidedly incongruous. In order to give the term utility in distinguishing between different types of behavior, I shall limit learning to the modifications in the behavior of an animal capable of forming images, when such modifications are due to this ability to form images. An animal is able to form images when it retains a memory of the experiences through which it passes. Here again it is necessary to limit the meaning of the word memory. It is contended by some that all organisms have memory because of the great plasticity of organic matter. But I believe that it is better to restrict the use of the term to the ability to reproduce or reconstruct in some fashion an experience through which the animal has passed. This does not result from the general plasticity of protoplasm, which cannot reproduce the experience through which it passes, though it may be modified by it. For memory to exist, a special organ of memory is needed, and this organ is to be found in the association areas of the cerebrum. As we have seen, impressions received through the senses are recorded upon these areas, so that later on when these areas are again stimulated the sensations will again be revived in part, if not in full, without the original causes of the sensation being present.

The process of learning, then, consists in part in forming these images of sensations experienced. It is evident that these images must influence behavior. If an image is of a sensation which gave rise to an act, it will be connected with the motor impulse which went to the motor organ. 
When, therefore, this image is stimulated in the future, it may in turn stimulate the motor center, which will send an impulse to the motor organ and thus give rise to the same act. This is ideo-motor action, which follows upon an image and not upon a stimulus from without, as is the case in sensori-motor action. It is evident that ideo-motor action is possible only to animals that can form images, so that the whole range of ideo-motor action is peculiar to these animals.

But the act connected with an image will not necessarily follow when that image is aroused. That will depend in part upon what has been the result of that act in the past. If the effect of the act has been pleasing, the path from the motor center to the end organ will be reënforced, so to speak, so that the act is more likely to be repeated. If the effect of the act has not been pleasant, the pathway to the end organ will be weakened or blocked, so that the act is not so likely to be repeated. This brings us to the phenomena of pain and pleasure which play so important a part in the determination of the behavior of the higher animals. The problem of the nature of pain and pleasure is closely connected with that of consciousness, so that it will be discussed in connection with consciousness. I will say here, however, that these phenomena seem to be characteristic of nervous matter and are apparently never experienced apart from such matter. Presumably they arise out of the states of the neurones involved. If the effect of a certain act upon the states of the neurones involved is harmful, the pathway to the end organ which caused that act will probably be weakened so that the act is not so likely to take place again. This weakening will probably be by a 
weakening of the synapses between the neurones along the pathway to the end organ. But we do not yet know enough about the inherent nature of the nervous system to understand just how and why these reënforcements and inhibitions of acts take place in the nervous system. If we did know, we would understand much better how these modifications in behavior which constitute intelligence come about. As Thorndike has said: "We may, therefore, expect that when knowledge of the structure and behavior of the neurones comprising the connection-systems of animals (or of the neurones' predecessors in this function) progresses far enough to inform us of just what happens when a connection is made stronger or weaker and of just what effects satisfying and annoying states of affairs exert upon the connection-system (and in particular upon the connections most recently in activity) the ability to learn will show as true an evolution as the ability to sneeze, oppose the thumb, or clasp an object touched by the hand." 1

We can now see that the process of learning includes the strengthening and weakening of associations as well as the formation of images. These two factors alone will introduce a large amount of uncertainty into the behavior of the animal. The observer of its behavior cannot know what images are recorded in its brain. Nor can he know all the pleasurable and painful feelings it has experienced and the associations which have been established by them. And yet a complete knowledge of both these things as well as a knowledge of the inborn tendencies to action of the animal is necessary to explain its behavior.

1 E. L. Thorndike, Animal Intelligence, New York, Igrr, p. 280. 


\section{The Forms of Intelligence}

These two factors are probably all that play a part in the intelligent behavior of all but a few of the higher animals. But in these higher animals these processes become much more complex, thus making much more uncertain the behavior of the animal. As the association areas grow and the radiation of association fibers becomes more refined, these images become related to each other in a very complex fashion. Thus there arise the higher forms of intelligence. The first of these is the idea. This term is used in somewhat different ways. Frequently it is used in such a fashion as to imply a high order of intelligence, as when "a man of ideas" is spoken of. But the more technical psychological meaning is much broader, as indicated in Baldwin's Dictionary, which defines an idea as being "the reproduction, with a more or less adequate image, of an object not actually present to the senses." It is evident that according to this definition an idea is little if anything more than an image, though sometimes an idea may be formed by the association together of several images. Ideas therefore may be held by animals which have not yet attained a very high grade of intelligence.

Thought is the next higher form of intelligence. It is not, however, a distinct thing in itself, but is simply the name for a flow of ideas, so to speak, which do not necessarily give rise to any external action. That is to say, an idea stimulates another idea and that one still another one, and so on, by means of the connections between their nerve centers in the associative tracts of the cerebrum, so that thinking is the name given to the mental process which is 
the result from or the concomitant, as the psycho-physical parallelists say, of this cerebral process. In order to have thought, therefore, it is necessary to have a large supply of ideas which are much interrelated. A higher grade of thought is conceptual thought in which concepts constitute the flow of thinking. A concept is a generalized image of a characteristic common to a number of images. It is evident that according to these definitions thought is made up of images. Introspection on the part of most people reveals images at the basis of all ideas and of all thinking. These images may be visual, auditory, tactile, kinæsthetic, etc., according to the nature of the sensations they reproduce. The introspective method is of great value at this point as throwing light upon the origin and nature of these phenomena.

Strange to say, however, there are a number of psychologists who have recently claimed the existence of imageless thought. Among them are to be found such writers as Stout, Binet, Woodworth, Buhler, etc. Their claims have, however, aroused much opposition among other psychologists, and a bitter controversy is now raging over this question. Already replies have been made to the supporters of imageless thought by Wundt, Titchener, Angell, etc., and it is probable that more criticism of their ideas will be forthcoming soon. There is not the space to review this subject fully in this place, so that I shall be able to make only a few comments.

The advocates of imageless thought seem to have introspective evidence of the existence of such thought. It is true that some of the subjects of their investigations have claimed to have had.ideas and other forms of think- 
ing independent of imagery. It is also true that some of these subjects have been trained psychologists. But error and self-deception are possible, even in the case of the trained subject. It may even happen sometimes that the trained subject is more likely to be deceived than the untrained one, for he is more likely to be looking for something in his mental processes, and may therefore deceive himself into thinking that he has found what he was looking for. This may explain why some of these trained subjects thought they experienced imageless thought. In any case it is well to bear in mind that mistakes may be made in introspection, even by trained observers. It is evident that imageless thought is quite contrary to the fundamental principles of modern psychology, so that very weighty evidence in its favor must be found before it can be accepted generally by psychologists. In the meantime we are safe in assuming that all thinking is done in images.

The highest form of intelligence is reason, which Baldwin's Dictionary defines as being "that faculty and process of mind which consists in the drawing of inferences." A large store of ideas is necessary for the appearance of reason. Then in the course of the flow of thought from one idea to another, similarities and differences between them will be recognized or other relations established which constitute reasoning. As I have already indicated, many have thought that reason is peculiar to man. If it is meant by this that there is not continuity in mental development from the lower animals to man, then reason cannot be regarded as peculiar to man in that sense. But if it is meant that no other animal has a sufficiently large store of ideas to furnish material for reason, then it may be that reason is to that extent peculiar to man. 
It may then be that man is the only animal that possesses reason, and the same is in large part true of the other higher forms of intelligence. I have already discussed briefly how the evolution of man's superior intelligence can be traced up through the lower mammals and the primates to man. We have seen that it is due in part to the superiority of certain of his senses which are of peculiar value in acquainting him with his environment, inasmuch as they are the functions of distance-receptors. It is due in part to his action-system, which enables him to go through an unusually varied number of movements. It is due in the last place to his extended association areas, which furnish the basis for an unusually extensive and complicated system of connections between sensations, images, and movements. It is interesting to observe how the monkeys and the anthropoid apes mark the development and the transition along all these lines from the lower mammals to man. ${ }^{1}$

But, as has been said, it is impossible to discuss fully. the higher forms of intelligence apart from consciousness. So that I shall now take up the discussion of consciousness.

1 Thorndike has summed up well the causes of man's superior intelligence in the following words: "The peculiarly human features of intellect and character, responses to elements and symbols, are the results of : first, a receiving system that is easily stimulated by the external world, bit by bit (as by focalized vision and touch with the moving hand), as well as in totals composed of various aggregates of these bits; second, of an action-system of great versatility (as in facial expression, articulation, and the hands' movements); and third, of a connection-system that includes the connections roughly denoted by babbling, manipulation, curiosity, and satisfaction at activity, bodily or mental, for its own sake; that is capable of working in great detail, singling out elements of situations and parts of responses; and that allows satisfying and annoying states of affairs to exert great influence on their antecedent connections. Because he learns fast and learns much, in the animal way, man seems to learn by intuitions of his own." (Op. cit., p. 28I.) 


\section{CHAPTER XV}

CONSCIOUSNESS : SENSATION, ATTENTION, FEELING, PLEASURE, PAIN, AND EMOTION AS CONSCIOUS ELEMENTS

Spiritual conceptions of consciousness, 282. - Consciousness as characterizing all matter, 283. - Consciousness as characterizing all organic matter, 283. - Consciousness as an epiphenomenon, 287. - Consciousness as associative memory, 288. - The neural basis of consciousness, 288 . - The relation between intelligence and consciousness, 289. - Sensations as the raw material for consciousness, 290. - The nature of attention, 290. - The nature of feeling, 291. - Pleasure and pain, 292. - Feelings as pleasurable and painful sensations, 292. - The neural basis of feelings, 292. Feelings of pleasantness and unpleasantness, 298. - The nature of emotion, 299. - The visceral and vasomotor origin of emotions, 300. - Emotions as affective sensations or feelings, 30r.

THE term consciousness is closely associated with the terms mental and psychic, and it is very difficult to find any definitions of these words, which give any concrete idea of their meaning. A curious example of this is to be found in Baldwin's Dictionary of Psychology and Philosophy in the definitions of the words consciousness and mind. In that work consciousness is defined as being "the distinctive character of whatever may be called mental life." Mind is defined as being "the individual's conscious process, together with the dispositions and predispositions which condition it." In other words, consciousness is defined in terms of the mental, while mind is defined in terms of the conscious, so that no concrete indication is given of the 
meaning of these words, if they have any. ${ }^{1}$ I shall therefore try to make my own analysis of consciousness on the basis of the preceding study of the different forms of behavior. This analysis will be as far as possible objective, though a little introspective data will be used.

\section{DefintTions of Consciousness}

It will be impossible to discuss all the different conceptions of consciousness which have been held in the past, many of which are still held, but a very brief statement will be made of some of the principal ones. Many writers in the past and a few still in the present have regarded consciousness as something spiritual and mystical in its character which resides in certain animals or in all living beings. Usually these writers have regarded it as a distinct entity or category which enters the body at the beginning of life and leaves it at death. Such theories are closely related to the religious doctrine of the soul and regard consciousness as something entirely distinct from matter. It is hardly necessary to state that no such theory of consciousness can be regarded as scientific because there can be no inductive evidence of the existence of any such spiritual entity. Furthermore, it denies the fundamental postulate of science that all things must be reduced as far as possible to the same terms, and assumes an ultimate dualism in the universe.

1 This dictionary was written by a considerable number of collaborators; so that it would not be surprising to find contradictions and such a circular method of defining as is illustrated in the definitions quoted above, if the definitions were taken from articles written by different persons. But the two articles from which the above definitions are quoted, namely, the articles on consciousness and on mind, are both of them signed by the same authors, namely, G. F. Stout and J. Mark Baldwin. 
On the other hand, there have been those who have carried this postulate of science to an absurd extreme by trying to prove that consciousness characterizes all matter. It is evident that if the term is to mean anything and is to be useful in the work of science, which is to describe natural phenomena, its application must be limited somewhat. This attempt to prove that all matter is conscious has been made by certain monists, and James has contended that such monism represents "reality" as having two irreducible aspects, the conscious and the material, so that it results practically in dualism. He goes on to show that when consciousness is represented as being fluid, unextended, diaphanous, and without content, it is a chimera, and that the term means nothing unless it includes concrete realities. ${ }^{1}$

Others have formulated more scientific theories to the effect that all organisms are conscious. I will give a few examples of such theories. Many of those who have held such theories have apparently been influenced principally by these two considerations. In the first place, in view of the continuity in the development of behavior, there is no place where consciousness could suddenly have made its appearance, and therefore must have been present at the beginning. In the second place, because of the analogies which exist between human behavior, which is undoubtedly conscious, and the behavior of the lower animals, all behavior must be conscious. For example, Jennings seems to have been greatly influenced by both of these considerations and especially by the second one, so that he is inclined to think that the behavior of all organisms is conscious. After

${ }^{1}$ La notion de conscience, in the Archives de psychologie, Vol. V. 


\section{The Science of Human Behavior}

raising the questions, on the one hand, as to whether the behavior of the lower organisms seems to be conscious, or, on the other hand, as to whether it seems to be unconscious, he says: "If one thinks these questions through for such an organism as Paramecium, with all its limitations of sensitiveness and movement, it appears to the writer that an affirmative answer must be given to the first of the above questions, and a negative one to the second. Suppose that this animal were conscious to such an extent as its limitations seem to permit. Suppose that it could feel a certain degree of pain when injured; that it received certain sensations from alkali, others from acids, others from solid bodies, etc., - would it not be natural for it to act as it does? That is, can we not, through our consciousness, appreciate its drawing away from things that hurt it, its trial of the environment when the conditions are bad, its attempting to move forward in various directions, till it finds one where the conditions are not bad, and the like? To the writer it seems that we can." 1

It is evident that Professor Jennings is drawing analogies between the behavior of the lower organisms and the higher animals which are probably not justified. For example, he thinks that a paramecium experiences pain when it is injured and draws away from harmful things for that reason. We have already discussed the mechanism of such behavior, using Jennings' own investigations to a large extent as a basis, and have seen that it is the result of the action of external forces upon the organism, constituting a tropism. As I have already stated, and as will be discussed more fully later on, pain and pleasure seem to be states that

1 Behavior of the Lower Organisms, New York, I906, p. 336. 
characterize the nervous system, so that it is very doubtful if they exist in organisms without a nervous system. Here again, as we have found to be true elsewhere in his writings, Jennings is carrying to an extreme his idea of the continuity in behavior and by so doing is destroying the utility of the word consciousness. It is evident that if all organisms are regarded as being conscious, the term means no more than sentiency, which is itself a very vague term, probably meaning no more than a high degree of plasticity arising out of the complex chemical composition of protoplasm. So that if the term is to have any utility in distinguishing between the different types of behavior, we must limit its meaning somewhat.

As a matter of fact, the continuity theory proves too much. If its upholders refuse to admit that consciousness made its appearance in the course of organic evolution, because of the continuity in the development of behavior, then why do they think that it appeared at the moment of transition from the inorganic to the organic? If they are thoroughgoing evolutionists they must believe that there was no absolute break at this point and that therefore there was no more reason for consciousness to appear at this point than later on. In other words, the logical conclusion of their theory would be that all matter is conscious, and this, as we have seen, is absurd.

This idea that consciousness characterizes all life has been very widespread and has manifested itself in many forms. Some seem to think that it is the essential principle of life. Others think that it is something entirely distinct from the life principle, which however it invariably accompanies. This is the parallelist theory that mental 
and organic processes accompany each other, without, however, affecting each other. It may be that some parallelists do not believe that mind accompanies all manifestations of life, but only some of the more complex of these manifestations. A curious variant of this theory is that the early organic movements were conscious, but on becoming habitual lost their conscious character and were inherited as unconscious reflex actions. We have already met this theory in an earlier chapter in the lapsed intelligence theory of instinct held by Lewes and Romanes. There seems to be some evidence in favor of this theory in the fact that conscious acts may on becoming habitual become unconscious and thus simulate the appearance of reflexes. But, as we have seen, no such acquired habit can be inherited, so that it is absurd to suppose that any inherited mode of action could have had a conscious origin. A singular illustration of this theory is to be found in one writer who tries to show that the movements which express emotions in man are inherited from the conscious movements of man's ancestors. For example, he says: "The face of proud contempt reflexly 'curves a contumelious lip.' What does the movement mean? Why, it lays bare the canine teeth; it is the human counterpart of the snarl of dog or wolf; it is the last reflex or unconscious remnant of a coördinated or impulsive action which, somewhere or other in our not remote ancestry, preceded the movements of actual attack. The deer bounds away when it hears the hounds, and we 'jump' when we are startled; the sitting bird crouches on its nest when danger approaches, and we wince or shrink when we are frightened or censured. . . . Here is evidence of the derivation of 
unconscious from conscious movement, not in the life history of the individual, but in that of the race." 1 In view of the preceding discussion of instinct, it goes without saying that these movements were no less unconscious and quite as reflex as they are in the human and other beings of to-day.

There is not the space to discuss at greater length these theories of consciousness, but I think that enough has been said to show that they are untenable. As has been shown, it is contrary to the fundamental postulate of science that all phenomena must be reduced as far as possible to the same terms to assume that consciousness is anything fundamentally different from anything else in the universe. Furthermore, to assume that consciousness is something that characterizes all matter is to identify it with energy, which is the universal characteristic of matter. In similar fashion, to assume that it characterizes all organic matter is to identify it with sentiency, so that in both cases the term loses its utility when it is applied so extensively. We must therefore, in the first place, determine if there is anything which can be distinguished as constituting consciousness, and, in the second place, must devise an objective, concrete criterion for it.

But before beginning this task I must discuss briefly the theory that consciousness is an "epiphenomenon." I believe that Huxley was the first to call consciousness by this name. I suppose that he meant by it that consciousness is not quite a phenomenon, but that it is something that accompanies certain organic phenomena.

\footnotetext{
1 E. B. Titchener, Were the Earliest Organic Movements Conscious or Unconscious? in the Pop. Sci. Mo., Vol. LX, p. 467 (March, 1902).
} 
It is probable that he gave it that name either because he did not think that it existed or because he despaired of being able to describe it as phenomenon. Now it goes without saying that science cannot recognize anything apart from phenomena, meaning by a phenomenon something which appears to the senses, so that either consciousness does not exist at all for man or else it exists as a phenomenon. So that I shall try to describe it as a phenomenon.

What, then, is consciousness? One of the best known attempts to define it objectively is the one which identifies it with the associative memory. For example, Loeb defines it as follows: "Consciousness is only a metaphysical term for phenomena which are determined by associative memory." " We have already seen that many regard associative memory as the mark of intelligence, which fact suggests that consciousness and intelligence may turn out to be the same thing, or at any rate that one of them is an aspect of the other. This is a possibility which I shall discuss shortly.

If consciousness is determined by associative memory, then it cannot appear until this memory has appeared. We have seen that associative memory is based upon the association areas of the central nervous system and that these areas have had their greatest development in the vertebrates. In the higher invertebrates, such as the articulates, the central nervous system is a segmented chain of nerve tissue which is dominated by the metamerism of the body and in which the reflex arcs of the different segments are kept more or less apart by the anatomical structure

1 Comparative Physiology of the Brain and Comparative Psychology, New York, I900, p. I2. 
of the animal. But in the vertebrate the central nervous system becomes an epithelial tube which is imperfectly segmented and which contains a continuous column of nerve cells and connecting fibers which forms a sort of reticulum. This reticular formation is a diffuse correlation center which relates together all of the reflex arcs. The anterior part of this epithelial tube expands into the brain, in which there are the special correlation centers or suprasegmental apparatuses, as they are sometimes called, which determine most of the mental phenomena of the higher vertebrates. Consciousness, then, like intelligence, will appear when the appropriate neural basis for associative memory has evolved sufficiently.

It must now be evident that this conception of consciousness which connects it so closely with the associative memory implies that it is a form of behavior or, to say the least, a characteristic of certain forms of behavior. This idea is suggested in the following definition formulated by Herrick: "Consciousness is a functional phase of the more complex mechanism of those higher nonstereotyped actions for which the reflex machinery is inadequate, in much the same way that the tropisms of Paramecium and the sucking reflex of an infant are functional phases of the simple inborn neuromuscular mechanisms of these organisms." 1 This definition seems to place consciousness in the same category with tropisms and reflexes with which we are acquainted as forms of behavior. But if this is true, then consciousness and intelligence must be identical, for intelligence is the functional phase of these higher, nonstereo-

${ }^{1}$ C. J. Herrick, The Evolution of Intelligence and Its Organs, in Science, Vol. XXXI, No. 784 (Jan. 7 , I9IO). 
typed actions. As a matter of fact, the relation between the two is very close, and they are undoubtedly at least partially identical. But it is not certain that they are wholly identical, and it may be that each includes phenomena which do not belong to the other. I have described briefly intelligent phenomena and will now discuss the contents of consciousness. After doing so, it will be possible to determine to what extent they are identical.

\section{Sensations and Attention}

We have seen that sensations are the impressions made upon the nervous system by stimuli which are received through the sense organs. It must be evident that there can be no consciousness without sensations. As Spencer pointed out, they furnish the raw material for consciousness. But sensations do not necessarily involve consciousness. If these sense impressions do not reach the higher nerve centers so that the acts which result from them are sensorimotor, it is inconceivable in view of what has been said that they are accompanied by consciousness. But if they do reach the higher nerve centers they may become conscious. In order to become so, they must be connected in the association areas with the images of the same or of other sensations. However, this alone may not be sufficient to render the sensations in question conscious. If other sensations are being received at the same time, it may be necessary for attention to be centered upon these sensations in order to make them conscious. By attention I simply mean that the nervous system responds to certain sensations, to the total or partial exclusion of other sensations which are being received at the same time. Now this may be due to 
several causes. It may be due to the fact that certain sensations are much more powerful than the others being received at the same time. Or it may be due to the fact that these sensations, though weak as compared with the other sensations being received at the same time, are connected with images which are sufficiently numerous or sufficiently vivid to monopolize wholly or in part the capacity of the nervous system for responding.

Is then the concentration of attention upon certain sensations an indication of the presence of consciousness? Some psychologists seem to think so. It has been indicated that Jennings believes that attention in the lower organisms is an indication that they are conscious. It is true that these organisms will attend to certain stimuli to the neglect of others which are acting upon them at the same time. This is due either to the fact that these stimuli are stronger than the others or to the fact that they have acquired the habit of responding to these stimuli. But this does not involve the presence of images and ideas as in the higher animals, and it is practically certain that this sort of attention cannot be regarded as any indication of the presence of consciousness. Hence it is that attention is not necessarily an indication of the presence of consciousness, unless we limit the term attention to concentration which involves a complex combination of images and ideas.

\section{Pleasure and Pain}

The next element in the contents of consciousness is feeling. This is an exceedingly difficult subject to discuss, since there is still great difference of opinion among the psychologists as to the nature of feeling. But I think this 
much may be safely said to start with, that in all probability feelings fall into two main classes, namely, pleasurable and painful sensations. Feelings which seem to belong to neither of these classes appear so because they are very close to the borderline between the two. Assuming, then, that all feelings are either pleasurable or painful, the problem of the nature of feeling reduces itself to the problem of the nature of pleasure and pain.

There have been numerous theories as to the nature of pleasure and pain. Most of them have taken account of the fact that pleasurable acts are usually beneficial to the organism, while painful acts are usually harmful. This fact indicates that selection has been at work preserving the pleasurable acts and feelings and eliminating the painful acts and feelings. But it is still very uncertain as to what is the exact mechanism of pleasure and pain. As has been stated, these feelings are never experienced apart from the nervous system, so that we have reason to believe that their mechanism is nervous in its character. Evidence of this is to be found in the fact that in the higher animals which display these feelings they can never be stimulated in those parts of the organism which do not contain nerve fibers. For example, however much the hair, the nails, and other bony parts of the body are injured, no feeling of pain can be aroused.

Furthermore, there is reason to believe that these feelings do not appear until the nervous system has reached a relatively high stage of development. We have evidence that the species which do not possess a highly developed central nervous system do not display these feelings, or display them only to a slight extent. Norman has gath- 
ered together numerous examples which indicate that such is the case. ${ }^{1}$ For example, if an earthworm is cut in halves, only the posterior half squirms and jerks as if in pain. The anterior half crawls away as if nothing had happened. And yet this is the half which contains the brain, which, as the highest part of the nervous system, should be most sensitive to painful stimuli. Furthermore, if the two halves are subdivided, it again happens in each case that the anterior part crawls away, while the posterior part squirms and jerks. The same thing happens as frequently as the parts are subdivided. It appears then that the squirming and jerking of the posterior pieces cannot be due to pain, for if this were the case these movements would be exhibited as much by the anterior pieces. It is probable that the difference in the movements of the two parts is due to the fact that when the impulse from the injured spot travels forward it affects the circular muscles first, thus causing them to contract and the part to elongate and to move forward, while when the impulse from the injured spot travels backward it affects the longitudinal muscles, thus causing the part to squirm and jerk.

The same phenomenon takes place when the worm Nereis is divided. When certain worms such as the Cerebratulus, Thysanozoön and Planaria torva, and the leech are divided, the posterior half in each case ceases movement, while the anterior half moves forward as if nothing had happened. Among the echinodermata the starfish and the brittle star can be cut to pieces without giving any appreciable reaction. Among the crustacea the legs and abdomen of the

${ }^{1}$ W. W. Norman, Do the Reactions of the Lower Animals Against Injury Indicate Pain Sensations? in the Am. Jour. of Physiology, Vol. III, No. 6 (Jan., 1900). 
hermit crab, the spider crab, etc., can be cut away without any movements which signify pain. Among insects the abdomen of bees, dragon flies, etc., has been cut away while the creature was eating without disturbing the process of nutrition, which seemed to indicate that there was no pain, or that the pain was so slight as not to interfere with the nutritive process. Among vertebrates vivisection experiments upon fishes such as sharks, flounders, etc., have not produced reactions which seemed to indicate pain. ${ }^{1}$

It goes without saying that pleasure and pain are phenomena which can be observed directly only by means of introspection. But in this respect they are like all other mental and psychic phenomena, for all such phenomena can be observed directly only by introspection. It is possible to judge the mental and psychic phenomena of others only by inference. When we see behavior in others which is in us the accompaniment of certain mental and psychic phenomena, we assume that these others are experiencing the same mental and psychic phenomena. In this fashion we can judge as to manifestation of pain and pleasure by other species. If when injured they do not manifest any reactions at all, or do not manifest the sort of reactions which we give when pained, we have some reason to believe that they are incapable of experiencing painful feelings. To be sure, this is not a sure sign, for it may be that the reactions in other species may be very different from what they are in man. But in the cases cited above, either no

\footnotetext{
1 Cf. W. W. Norman, Do the Reactions of Lower Animals Due to Injury Indicate Pain-Sensations? in Biological Lectures from the Wood's Holl Marine Biological Laboratory, Boston, I899; also in Pflüger's Archiv, Bd. LXVII, p. I37; Loeb, op. cit., pp. 229-232; W. M. Wheeler, Ants, New York, ıو10, p. 509.
} 
reactions whatever were given when injury was inflicted, or else the reactions were of such a nature as could be fully explained on other grounds than as manifestations of painful feelings. When, however, we come to the higher vertebrates, and especially the mammals, we find unmistakable evidence of pleasurable and painful feelings. These are movements of drawing away from painful stimuli and approaching pleasurable stimuli, the utterance of cries and other sounds, the expression of the face in some of the higher animals, etc.

Feeling is then apparently limited to animals with a central nervous system which is quite highly developed. As has been indicated, there have been many theories as to the mechanism of feeling. Spencer propounded the theory that pleasure is the concomitant of heightened nervous discharge, while pain is the concomitant of lessened nervous discharge. Later, Bain and Baldwin proposed similar theories as to the physiology of pleasurable and painful feelings. According to these theories, pleasurable acts are more likely to be repeated, because the heightened nervous discharge which has accompanied them has opened up the paths to the motor organs involved and has reënforced the tendency to perform these acts. Painful acts are less likely to be repeated, because the lessened nervous discharge which has accompanied them has, to say the least, not opened up the paths to the motor organs involved, while it may even have an inhibitory effect upon them. But it is doubtful if these theories are correct, for it seems quite evident that painful acts are accompanied by as heightened a nervous discharge as pleasurable acts frequently, if not always. Certainly the vigorous reactions 
usually given to painful feelings would seem to indicate that such is the case. Furthermore, if this theory were true, it would be reasonable to suppose that pleasure and pain would arise very low in the animal scale, for there could be this difference in the strength of the nervous discharge as soon as there were any nervous currents. To be sure, it is not absolutely certain that pleasurable and painful feelings are not experienced very low in the animal scale, but $\mathrm{I}$ have given reasons for believing that such is not the case.

Another theory is that painful feelings are caused by special organs for pain. That is to say, certain nerve fibers are specialized for the purpose of giving warning against harmful stimuli, and the sensations received through these organs are those which we call painful feelings. These special organs for pain are distributed throughout the organism according to need. Those who hold this theory usually believe that pleasurable feelings are diffused forms of sexual sensations. According to this theory it is easier to believe that painful and pleasurable feelings do not appear until relatively high in the organic scale, at least so far as painful feelings are concerned, for these feelings. could not appear until the appropriate organs for them had developed. According to this theory it is also possible to believe that one kind of feeling appeared before the other, and there is some reason for believing that painful feelings appeared before pleasurable feelings.

\section{Feelings}

But feelings have not been studied enough as yet to furnish a well-established theory as to their nature. Cer- 
tainly nothing definite can be learned from what are supposed to be authoritative sources of information. For example, Baldwin's Dictionary defines feeling as being "consciousness as experiencing modifications abstracted from (I) the determination of objects, and (2) the determination of action." 1 This definition is utterly meaningless to me, with the exception that it is apparently defining feeling in terms of consciousness. As we have already seen, this dictionary defines consciousness in terms of mind, and mind in terms of consciousness, so that nothing can be learned about consciousness in this dictionary. Consequently we have feeling defined in terms of what is, if anything, more unknown than itself. I am well aware that if we follow up the definitions of terms to the last stage of analysis, we are certain to run up against the unknown, for all our knowledge is relative and nothing is absolutely known. But it is well when defining a thing to do so if possible in terms of what is more concrete and better known. Now in the case of feelings I believe that we should begin with sensations and indicate that feelings are certain kinds of sensations or, at any rate, certain aspects of certain kinds of sensations. By doing so we should be defining feeling in terms of something that is more or less concrete and tangible and about which something is known. It may be that some will deny that feelings have anything to do with sensations. But it seems to me that those who make such a contention would be denying the determination of psychic phenomena by physical forces. Certainly those who believe in such physical determination of psychic phenomena

1 The article on feeling in this dictionary is signed by J. M. Baldwin and G. F. Stout, who, as has already been noted, also wrote the articles on consciousness and mind in this dictionary. 
must believe that all psychic processes are initiated by physical stimuli, and these stimuli affect the nervous system by giving rise to sensations. So that sensations furnish the basis for feelings as we have seen they furnish the raw material for consciousness and as indeed they are the basis for all psychic phenomena.

Some writers distinguish between feelings of pleasure and pain and feelings of pleasantness and unpleasantness on the ground that these latter feelings do not result directly, at any rate, if at all, from sensations caused by external stimuli. The theory held by some is that feelings of pleasantness and unpleasantness are caused by changes in the intensity of the nervous current and not by stimulations received through specific sense organs. ${ }^{1}$ This theory is, therefore, similar to Spencer's theory that all painful and pleasurable feelings are due to changes in the intensity of the nervous current. It is, however, a mistake to assume, as is sometimes implied, that because feelings of pleasantness and unpleasantness are not due to external stimuli, they are not based upon sensations and that they are radically different from painful and pleasurable feelings. Internal physiological processes may give rise to sensations just as well as external stimuli, so that if these pleasant and unpleasant feelings are due to the increase or decrease of the intensity of the nervous current they are quite as sensational in their origin as other feelings. Furthermore, as sensations with an affective aspect they cannot be radically different from other painful and pleasurable feelings. As we have seen, all feelings are in all probability pleas-

1 Cf. Max Meyer, The Nervous Correlate of Pleasantness and Unpleasantness, in the Psychological Review, Vol. XV, No. 5 (Sept. I908). 
urable or painful, and feelings of pleasantness and unpleasantness differ from other feelings only in that they are more diffused and less localized than feelings which are due to sensations which are received through specific sense organs.

Assuming, then, that feelings are certain kinds of sensations, or, to say the least, certain aspects of certain kinds of sensations, it must be recognized that they play an important part in the determination of behavior. As we have seen, painful feelings tend to inhibit the acts which give rise to them or to draw the animal away from the stimuli which cause them, while pleasurable feelings tend to reenforce the acts which give rise to them and to draw the animal towards the stimuli which cause them. Feelings, then, are of importance, in the first place, as factors in the determination of behavior, and, in the second place, as forming a part of the contents of consciousness. Let us see what other psychic phenomena are included in the contents of consciousness.

\section{EMOTIONS}

The emotions are usually regarded as important elements in consciousness. Later on I shall give reasons for thinking that the emotions are feelings. But they have usually been discussed by themselves, apart from the feelings, and some writers seem to think that they are quite distinct from the feelings. For these reasons I shall discuss them by themselves at first and then discuss their relations to other feelings.

There have been various theories in the past as to the nature of emotions. Some have thought that there are 
special organs for emotions which are stimulated to function by external forces. But it is now pretty generally believed that the emotions accompany certain tendencies to action and are the by-products, so to speak, of these tendencies. This is the so-called James-Lange theory of the emotions, to which I have referred when discussing instinct. It is doubtful if a simple reflex action could be accompanied by an emotion, since the organic processes involved are not sufficiently complex. The instincts, therefore, are these tendencies to action which are accompanied by emotions. As James has said, "an emotion is a tendency to feel, and an instinct is a tendency to act characteristically when in the presence of a certain object in the environment."

The great question is as to how these tendencies to action give rise to emotions. Both James and Lange believed, as we have seen, that emotions are the incidental results of the organic reaction. But James thinks that they result from the effect of the organic reaction on the viscera, while Lange thinks that they result from the effect of the organic reaction on the vasomotor or vascular system. Certainly the part played by the viscera in the emotions is quite evident, as illustrated in the heightened beating of the heart in anger and other emotions, the contraction of the blood vessels causing a blanching of the skin in fear and the expansion of the blood vessels causing their flushing in shame, the stimulation of the bowels in fear, etc. But it is likely that the vasomotor system also plays a part. An illustration of this may be the secretion of the lachrymal gland in grief. But there is not the space to discuss here the relative importance of the parts played 
by the viscera and the vascular system in emotions. In the case of both the process which takes place must be somewhat as follows: We have seen that almost all the stimuli to action in animals with a nervous system come through this system. If action takes place, however, there is involved the coöperation of the muscles, viscera, vascular system, etc. The movements of these parts of the organism must react upon the nervous system to a certain extent, and the emotions appear to be some, if not, all of the results from this reaction. The reaction is principally upon the sympathetic system, which furnishes most of the nerves for the viscera and vasomotor systems. The emotions, then, are the feelings which are aroused in the nervous system by these internal processes, and the movements of muscles, the viscera, etc., which accompany the emotions are their causes.

It is true that emotions will sometimes arise when no external action takes place. One attempt to explain these cases was that of Darwin and Spencer, who thought that these were reminiscences of ancestral modes of behavior in response to certain stimuli where these modes of behavior have in the main disappeared. But the theory as stated by these writers seemed to imply the transmission by heredity of acquired habits which, as we have seen, is very doubtful. It might happen that part of an instinctive tendency to a certain mode of action would die out as a result of changes in the nervous system, so that the organic reaction to the appropriate stimulus would be only sufficient to give rise to the emotion, but not to the external action. But it is more likely that these cases where an emotion is aroused without any external act taking place are due to 
some inhibitory force which checks the act, but not the emotion. Thus an acquired habit of self-control may restrain an individual from striking when the instinct of pugnacity is aroused, but cannot inhibit the rise of the emotion of anger which is accompanied by increased heart action, tense muscles, etc. Self-control may prevent an individual from fleeing from an object of danger, but cannot prevent the emotion of fear which is accompanied by a contraction of certain of the blood vessels, trembling of the knees, etc. In fact, it sometimes happens that the emotion is strongest when the external act is inhibited, because action usually relieves the organic conditions which give rise to the emotion. This indicates how an emotion may reenforce and strengthen a tendency to an action in order to secure the relief which comes through action. This is why emotions become powerful factors in the determination of behavior.

But certain experiments have been made which seem to contradict this theory that the emotions are aroused by the reaction of the viscera and other parts of the organism. Sherrington ${ }^{1}$ has, by means of appropriate spinal and vagal transection in dogs, removed the sensation of the viscera and of all the skin and muscles behind the shoulder, and has destroyed the connection between the organs of consciousness and the whole of the circulatory apparatus of the body. Such animals have displayed the emotions of anger, joy, disgust, fear, etc., just as much as before the operation, thus seeming to indicate that these emotions can be aroused in the brain independently of the viscera, etc. Certain psychologists, such as Lloyd Morgan, ${ }^{2}$ An-

1 The Integrative Action of the Nervous System, New Haven, I906, pp. 255-268.

2 Animal Behaviour, 2d. edit., London, 1908, p. 92. 
gell, ${ }^{1}$ etc., have denied that these experiments have disproved the theory that emotions are caused by the reaction of the viscera and other parts of the body on the ground that the mechanism for these emotions had already been developed in these animals so that the emotions could be aroused independently of the viscera, etc. As Morgan puts it, "the avenues of connection were closed after the motor and visceral effects had played their parts in the genesis of the emotion on the hypothesis that the emotion is thus generated. Although new presentative data of this type were thus excluded, their re-presentative aftereffects in the situation were not excluded." Sherrington, however, has replied by stating that one of the dogs was but nine weeks old when the operation took place, and yet this animal displayed disgust when offered dog's flesh to eat. He thinks that it had not lived long enough for this emotion to be fixed in the brain so that it could be aroused independently of visceral stimulation. As he puts it, "disgust for dog's flesh could hardly arise from the experience of nine weeks of puppyhood in the kennel."

As a result of these experiments, Sherrington is inclined to believe that emotions originate in the brain and not in the viscera. He says: "We are forced back toward the likelihood that the visceral expression of emotion is secondary to the cerebral action occurring with the psychical state. There is a strong bond of union between emotion and muscular action. Emotion 'moves' us, hence the word itself. If developed in intensity, it impels toward vigorous movement. Every vigorous movement of the body, though its more obvious instrument be the skeletal

1 Psychology, Naw York, I908, p. 37 I. 
musculature of the limbs and trunk, involves also the less noticeable coöperation of the viscera, especially of the circulatory and respiratory. The extra demand made upon the muscles that move the frame involves a heightened action of the nutrient organs which supply to the muscles the material for their energy. This increased action of the viscera is colligate with this activity of muscles. We should expect visceral action to occur along with the muscular expression of emotion. The close tie between visceral action and states of emotion need not therefore surprise us."

Sherrington cites as further evidence of his theory the decerebration experiments of Goltz. Goltz kept alive for many months a dog from whom the hemispheres of the brain had been removed. During all this time it gave no sign of fear, joy, affection, or sexual emotion, but it repeatedly gave expression to anger and displeasure, both by gesture and by voice. This experiment seemed to indicate that a higher nervous organization is needed for the first set of emotions than is needed for anger and displeasure. Sherrington thinks that these experiments furnish evidence "that emotion is primarily a cerebral reaction." But I do not think that this is necessarily the case, for the brain is just as necessary for emotion as a psychic phenomenon if the stimulus which gives rise to it comes from the viscera as it is if the emotion originates in the brain independent of the viscera. I do not suppose that any one who believes in the visceral or vascular origin of emotions thinks that these emotions are actually felt in these organs, but that, on the contrary, all are agreed that all emotions are felt in the nervous system, and that as 
psychic phenomena most, if not all, emotions manifest themselves in the brain.

It must now be evident, I think, that more experiments must be made before we can hope to know with certainty just where emotions originate. I believe, however, that we can say with certainty that the viscera and other internal organs and the vasomotor system are involved in emotions as well as the nervous system. Sherrington expresses this opinion from his point of view in the following words: "In view of these general considerations and of the above experiments, we may with James accept visceral and organic sensations and the memories and associations of them as contributory to primitive emotions, but we must regard them as reënforcing rather than initiating the psychosis. Organic and vascular reaction, though not the actual excitant of emotion, strengthen it."

I think that the preceding discussion of emotion must have shown that emotions arise out of sensations. The stimuli for these sensations may not come from outside, but they are none the less sensations. Many of them are kinæsthetic sensations which are caused by movements of the muscles. Moreover, they are sensations with a distinctly affective aspect. That is to say, they are all of them sensations which are either pleasurable or painful and can be classified as such. If this be true, then the statement made earlier in this chapter that emotions are feelings has been sustained. They are, however, usually more powerful than ordinary feelings and accompany and reënforce certain definite types of behavior, so that they play a larger part than ordinary feelings in the determination of behavior. Furthermore, they seem to impart an unusually rich "feel- 
ing-tone," as some psychologists say, to consciousness. It is quite likely that they have a longer history than any other element in consciousness, since they are to a large extent contemporaneous with the instincts. If so, they form the earliest group of feelings. I do not believe that they constitute consciousness when they stand alone, but when consciousness has made its appearance they add something to its character, as will be shown in the following chapter. 


\section{CHAPTER XVI}

PERSONALITY, INTELLIGENCE, CONSCIOUSNESS, AND THE NATURE OF MIND

Self-consciousness or personality based upon the integrated permanent psychic elements, 307. - Self-consciousness as an idea, 309. Multiple personality, 309. - The nature of volition, 31 I.-Subjective and objective aspects of consciousness, 313.- The relation between consciousness and intelligence, $3 \mathrm{I} 4$. - The criterion of consciousness, 314. - The functions of consciousness, 315.- Consciousness as self-consciousness, 3I9. - Definition of consciousness, 321 . Psycho-physical parallelism, 32r. - Psycho-physical interactionism, 322. - Definition of mind, 322. - Mind as including intelligence and consciousness, 322. - Mental processes determined by minute physiological processes, 322. - Mind as a stage in the determination of certain kinds of behavior, 323. - The relation between mind and matter, 323. - The criterion of mind, 324. - Is our knowledge of mind subjective or objective, 325 .

THE highest plane of consciousness is reached in self-consciousness or the sense of personality. Self-consciousness, like consciousness, is frequently spoken of as a distinct entity. But it is no more an entity than consciousness. In fact it is a less concrete and more intangible thing than the sensations, feelings, and emotions which have been discussed. We can best understand its nature by considering briefly the conditions under which it appears.

\section{SELF-CONSCIOUSNESS}

Self-consciousness is based upon the more or less permanent psychic elements. That is to say, it is essential for the ap- 
pearance of self-consciousness that there should be a certain number of sensations, feelings, and ideas which are more or less permanent. It is hardly conceivable that selfconsciousness could appear in an individual whose feelings and ideas were constantly changing so that his psychic equipment would become entirely different within a short interval of time. Assuming, then, such a more or less permanent equipment of psychic elements, self-consciousness appears as a result of the process of integration which gives to these diverse psychic elements a certain unity. This integration takes place by means of the same mechanism which performs the other types of integration which have been discussed, namely, the central nervous system and in particular the association areas of the brain. The kind of integration involved in personality is, however, different from that involved in instinct, for example, in that it is not inherited but is acquired in the course of the lifetime of the individual. This is to be expected of any kind of integration which grows in large part out of the association areas for, as we have seen, these areas are not specialized at birth but become so during the life of the individual. For the same reason consciousness in general is in large part if not entirely an acquired character, for it also grows in large part out of these association areas.

It is, of course, impossible to tell just when the sense of personality first made its appearance, but it has probably existed in a very rudimentary form in many of the higher animals. This rudimentary form is probably in the main a state of feeling. It arises out of the harmonious and more or less permanent relations with each other of certain feelings and images. Hobhouse has made a good attempt to 


\section{Personality, Intelligence, Consciousness 309}

describe it when he says that a dog or ape has "a self, i.e. a pervading identity and permanent character, is aware at least of its present needs and seeks to identify them. What we miss is evidence that the self is present to it as a persistent identity in such a way, for example, as to shape the choice of immediate ends by considerations of lifelong welfare. The self of which the animal is conscious is a very small fragment as compared with the self of which the man is aware." 1

But self-consciousness cannot develop very far until it becomes an idea, and its evolution as an idea has been principally if not entirely among men. To trace fully the development of the idea of personality would be to trace the whole course of human, mental, and social evolution. It is doubtful if the idea of personality could be conceived by an individual living in isolation. This idea grows out of the observation of the likenesses and differences between the observer and other individuals, so that it is in large part a social product. The development of language played an important part, for it gave to the idea of personality definiteness and clearness just as it has done so to all ideas. Unfortunately I have not the space to discuss this subject at greater length, but these few suggestions give a slight indication of the causes for the superior development of human self-consciousness.

That self-consciousness is not a distinct entity is indicated by a number of the phenomena which characterize it. For example, the phenomenon of so-called multiple personality is such an indication. Every personality is to a certain extent multiple. That is to say, each individual

1 Mind in Evolution, London, I90I, p. 312. 
possesses several different sets of feelings and ideas, each of which is stimulated by an appropriate environment or by suitable physiological conditions. But ordinarily these sets are not separated from each other so greatly but that the individual recognizes that they belong to the same personality. Under pathological conditions, however, the separation between these different sets may become so great that the individual does not recognize them as belonging to the same personality, so that there arise the phenomena of multiple personality and of mental alienation. Hobhouse indicates the varying degrees in which personality is multiple in the following words: "In a sense, it is scientifically true that the business man, who has spent the day in besting a rival, is not the same person as the father who comes home to romp with his children. The outward and visible semblance is the same, but within it there are two quite different masses of thought, emotion, and will, each forming an interconnected system by itself, yet standing in no logical or moral relation with the other. When the cleavage becomes extreme, we speak of 'double personality,' of alienation, or insanity. But the germ of this sort of madness is in all of us." 1

The effect of drugs and narcotics in disintegrating the consciousness of self temporarily and sometimes permanently is a significant indication that it is not a distinct and permanent entity. The way in which the sense of personal identity is lessened and sometimes changed in dreams is further indication of this fact. These phenomena cannot be explained on the ground of a subconscious self. ${ }^{2}$ To

1 Op. cit., p. 302.

${ }^{2}$ Cf. A. H. Pierce, An Appeal from the Prevailing Doctrine of a Detached Subconsciousness, in Studies in Philosophy and Psychology Dedicated to C. E. Garman, Boston, 1906. 


\section{Personality, Intelligence, Consciousness 311}

do so would be to assume the existence of two distinct, permanent entities, whereas we have seen that we have no reason to believe in the existence of even one such entity. These phenomena can be explained only on the ground of a unification of the contents of consciousness by the higher integrative mechanism of the central nervous system, which unification can, however, be destroyed in varying degrees or can be disintegrated into several smaller unities.

\section{VoLITION}

The will or volition is usually regarded as an expression of the self. There is not the space to enter upon an extended discussion of the many theories of the will which have been proposed, but I hardly need to say that science does not accept any theological or metaphysical doctrine of a will which is a distinct entity and which is independent of natural forces. No more can science accept a theory of will which regards it as immanent in the universe. So far as anything like will or volition exists at all it is a characteristic of consciousness and probably should be limited to self-consciousness. Volition manifests itself when the idea of an act, which has grown out of memory images of the act, and other contents of the memory influence behavior. Thus a stimulus to a certain action may be received, and the act will be performed unless the contents of the memory consciously inhibit it, in which case the volition will have been exercised. If the inhibition is unconscious as the result of habit, it cannot be regarded as volitional. Or the idea of a certain act may be aroused and serve as a stimulus to its performance, in which case a volitional act will have been performed. In each case of a volitional act the stimuli 
to action are either inhibited or reënforced by the contents of the memory and other characteristics of the individual, and the process is accompanied by consciousness, so that there frequently arises the illusion that the act is determined by the self, independent of external forces. Hobhouse has described the will very well in the following words: "The will is not to be regarded as an additional impulse, or as a force existing outside impulses and operating upon them. It is rather the system or synthesis of impulses, the broad practical bent and tendency of one's nature. Now here as elsewhere the development as we pass from a lower to a higher stage consists primarily in a growing explicitness. It is quite possible that even in animal life, when there is a conflict of desires, that one tends to prevail which is most intimately bound up with the animal's whole mode of life.... The new development is merely that those broad tendencies of the character which before operated, if at all, obscurely and unconsciously, have now a definite conception to guide them. The nature of the self, its character, its duties, the wider life of which it is a part, now become conceptions, ideals, or principles, which appeal to the personality as a whole, just as particular satisfactions appeal to special impulses. Just as the special impulse when it formulates its end into an idea becomes Desire, so what we have called the broad impulses of the personality, when their end is defined by conception, constitute Will." 1

\section{NATURE OF CONSCIOUSNESS}

The preceding has been a brief discussion of the contents of self-consciousness. The description of these contents 1 Op. cit., p. 313. 
has, I fear, sometimes been rather vague. But such vagueness is to a certain extent inevitable in connection with such intangible phenomena, and the same is even more true with respect to consciousness in general. I shall, however, endeavor now to set forth as definite a theory of the nature of consciousness as is possible with the known facts.

Many psychologists have asserted that consciousness is purely subjective, and a psychological definition of consciousness which made no attempt to define it in objective terms has been quoted. One psychologist specifically states that "consciousness we can only define in terms of itself." 1 Two questions may be raised in connection with this position. In the first place, does anything purely subjective exist? That is to say, does anything subjective, such as an idea or a feeling, exist entirely independent of an objective basis, such as a nervous system? It if does, we must accept a dualistic philosophy which, as we have seen, is hardly compatible with the standpoint of science which is trying to reduce all phenomena to the same terms. It seems, then, as if the subjective should be regarded as one aspect of the objective or as the objective looked at from a certain point of view. In the second place, if consciousness is purely subjective, can it be studied by science? It seems quite clear that tangible phenomena are necessary for any scientific study, so that if consciousness presents no such phenomena it is useless to attempt a scientific study of it. However, for the reasons given above, we are justified in believing that consciousness is objective if it exists at all, and that consequently it is possible to study it scientifically.

What, then, is consciousness as an objective phenomenon, if 
it exists at all? I have already suggested that it may be a form of behavior. If so it must be a form of movement and must involve the expenditure of energy. ${ }^{1}$ It cannot be an inherited form of behavior, but must be a variation from such behavior. I have already raised the question as to whether consciousness is identical with intelligence, which is a form of varied behavior. I have quoted Loeb's conception of consciousness as the activity of the associative memory which, according to him, is identical with intelligence. I have also quoted Herrick's, which also seems to identify the two. But I think the preceding discussion must have shown that the two are not identical. Consciousness cannot develop very far without the ideas which intelligence furnishes it and which constitute an important part of its contents. But consciousness includes the feelings as well, and it may be that consciousness originated in the form of feeling. This may be putting its origin too far back, and it may be that it did not appear until after the beginning of intelligence. It may even be best not to recognize consciousness until the idea of the self has made its appearance. But at whichever point we place the origin of consciousness it is evident that it contains something more than the ideas which belong to intelligence, so that the two are not entirely identical.

Some writers have regarded the ability to learn as the criterion of consciousness. ${ }^{2}$ It is evident that this would depend upon the answer to the question as to when consciousness originated which I have just discussed. If con-

1 Cf. W. P. Montague, Consciousness as Energy, in Essays in Honor of William James, New York, Ig08.

2 Cf. Mary W. Calkins, The Limits of Genetic and of Comparative Psychology, in the Brilish Journal of Psychology, Vol. I, Pt. 3 (Jan. 1905). 


\section{Personality, Intelligence, Consciousness 315}

sciousness appeared before intelligence, then the ability to learn would not always be a test of consciousness. If the two appeared at the same time, it would always serve as a test. If consciousness appeared after intelligence, a very slight ability to learn would not necessarily indicate the presence of consciousness. However, in most cases and for all practical purposes the ability to learn serves very well as a criterion of consciousness for, as we have seen, intelligence and consciousness accompany each other all the time, with the possible exception of the earliest stages of the one or the other. The same is true of varied behavior as a criterion of consciousness.

Let us see just how consciousness operates and how it influences behavior. It goes without saying that this is the principal reason for our interest in consciousness, and for that matter it is the principal reason for studying it, for if it did not influence behavior, it would be a waste of time to study it. Consciousness is of importance only as what Lloyd Morgan calls "effective consciousness," which is "that which enables an animal to guide its actions in the light of previous experience." 1

As we saw in the last chapter, Romanes; Spencer, and others believed that consciousness appears between stimulus and response. Herrick displays the same idea when he says that it makes its appearance between anticipation and consummation. "In the storm and stress of this interval just preceding the consummatory reaction the higher mental faculties are born." 2 Now by this is not meant, of course, that during this interval a spirit or fairy

1 Habit and Instinct, London, 1896 , p. 127.

2 The Evolution of Intelligence and its Organs, in Science, Vol. XXXI, No. 784 (Jan. 7, 1910), p. 13. 
appears which influences the response to the stimulus. But during this interval ideas and feelings may be stimulated and may influence the response and therefore the behavior which results from the stimulus. It is evident that in order to permit consciousness to appear such an interval must exist, and that the pathway from stimulus to response must not be so firmly fixed that nothing can change it. As Royce says, consciousness "attends those processes which, while involving the cortex, are of a decidedly complex grade and of a relatively hesitant character, or which come in consequence of the graver interferences on the part of our environment." 1 It is evident that these conditions can be found only in animals with a nervous system which is relatively highly developed and possesses the higher nerve centers. So that, as Meyer says, "consciousness accompanies processes in the 'higher' connecting neurons, the higher 'nerve centers,' whereas processes restricted to the lower centers may go on without any consciousness." 2 It is therefore a serious mistake when Claparède, who is a psycho-physical parallelist, says that "the question of the greater or less intelligence of animals no more prejudges that of their degree of consciousness than a concept of a tropism implies the absence of consciousness. These are two questions the solutions of which neither prejudge nor mutually exclude each other. We ought to oppose the simple to the complex, not the simple to the conscious." 3 On the contrary, the degree of consciousness does correspond directly to the degree of complexity of the neural mechanism.

But even in complex nervous systems there are a great

1 Outlines of Psychology, New York, 1904, p. 8x. $\quad 2$ Op. cit., p. 306.

Op. cit., p. 3 I4. 


\section{Personality, Intelligence, Consciousness 317}

many responses which are unconscious because the pathway from stimulus to response is firmly fixed so that there is no chance for variation. In fact it is very doubtful if even in man the number of conscious actions ever exceeds the number of unconscious ones. In the first place, there are the inherited modes of reaction which never become varied in the course of individual experience. This is especially true of the reactions controlled by the lower nerve centers. In the second place, there are the large number of acts which were at first conscious because they involved individual adjustment to the environment, but which have become habitual, that is to say, the pathway has become so firmly fixed that there is little chance of variation taking place, so that the acts are unconscious. It is this transition from conscious to unconscious behavior which has led certain writers to think that all behavior was at first conscious, but I have already pointed out the fallaciousness of this idea. It appears then as if consciousness acts in accordance with the law of parsimony. It appears when needed but disappears as soon as that need ceases. As Herrick has said : "Here we see how intelligence and feeling are developed as the servants of action. They do not appear so long as the action can be effected without them, and they vanish as soon as the reflex machinery of an adaptive action is set in motion." 1

Various attempts have been made to state just how consciousness influences behavior. For example, Minot says that "the function of consciousness is to dislocate in time the reactions from sensations." $2 \mathrm{He}$ apparently means

1 Op. cit., p. 16.

2 C. S. Minot, The Problem of Consciousness in its Biological Aspects, in Science, Vol. XVI, No. 392 (July 4, 1902). 
that it can delay the reactions to certain sensations. Later on he asserts that "consciousness has the power to change the form of energy, and is neither a form of energy nor a state of protoplasm," and that "the universe consists of force and consciousness." Apparently, therefore, he regards consciousness as a sort of mystical force quite distinct from natural phenomena. This conception is, as we have seen, quite fallacious. But his description of what is done by consciousness is more or less accurate so far as it goes, though this function is performed not by a mystical force but by the higher centers of the nervous system. It is, however, true, as has been pointed out by a number of critics, that this function may be performed by an unconscious mechanism. For example, an alarm clock is so constructed that it will delay for many hours the reaction to the stimulus of being wound up. So that this description of the function of consciousness is not quite adequate.

A more adequate statement of the function of consciousness has been made by Judd. ${ }^{1}$ He thinks that inner selfsufficiency and increasing autonomy of the individual, making it more and more independent of the environment, form the end toward which organic evolution is progressing. Consciousness plays a more and more important part in this process, and consequently he defines it as follows: "Consciousness is a function which promotes self-sufficiency by literally taking up the environment into the individual and there remoulding the absorbed environment in conformity to individual needs." He believes that it has become the principal factor in the life of man, going so far as to say

1 C. H. Judd, Evolution and Consciousness, in the Psychological Review, Vol. XVII, No. 2 (March, I9I0). 


\section{Personality, Intelligence, Consciousness 319}

that "consciousness is the essential fact in human life as I have attempted to show. What man does with his environment depends upon consciousness." It has, however, been the intellectual rather than the affective part of consciousness which has played the principal part. "Human behavior is not aimed at maintaining oneself within the environment, it is aimed rather at complete remoulding of the whole environment, and the chief instrument in this process of remoulding is intellectual comparison and deliberation, not emotion." Judd tends to regard consciousness too much as if it is an entity. For example, he says that "consciousness is a cause of events in the physical world." We have seen that we have no tangible evidence of such a thing as consciousness, and it is at most a name for certain relationships, or for a certain process. But in his discussion he gives a fairly good description of this process.

Consciousness, then, exists whenever behavior is influenced by ideas or by feelings. It is true that this statement still leaves the conception of consciousness rather vague. Our conception of it could be made more precise by limiting consciousness to self-consciousness, and there are a number of reasons why it would be well to do so. While it is impossible to determine just when the sense of personality first made its appearance, we can probably come nearer to it than we can to determining when consciousness in general first made its appearance. Furthermore, the sense of personality marks off a stage in mental evolution more definitely than does consciousness in general. A few psychologists have identified consciousness with self-consciousness. For example, Miss Calkins says: "Animals, if they are conscious at all, must be conscious of selves, for 
consciousness of any other sort is inconceivable. To be conscious simply means to be conscious of oneself in this or that or the other situation." 1 She goes on to explain that she does not mean the "definite, discriminated, reflective self-consciousness of the psychologist or philosopher," but a vague sense of personality possessed by young children and by some of the animals besides man. Royce also seems to have this idea when he says that "a state of consciousness exists when somebody is conscious of that state. When nobody is conscious of that state, it does not exist." 2 It may be that these writers think that the sense of personality originated as early as feelings and ideas, in which case their identifying consciousness with it does not make consciousness any more precise in meaning. ${ }^{3}$ If, however, they think it appeared later, to identify consciousness with it is to make the meaning of consciousness more precise.

Other psychologists have opposed the limitation of the term consciousness to the sense of personality. Thus Ward, who identifies consciousness with experience, says of consciousness that "it is continually confused with self-consciousness, which was its original meaning; and thereby the errors of intellectualism, which we have just discussed, are apt to be perpetuated and a part of experience mistaken for the whole." 4 It seems quite evident that he has in mind a well-developed idea of the self, while Miss Calkins

1 Op. cit., p. 284 .

2 Op. cit., p. 108 .

- Miss Calkins may mean to imply this when she says that all consciousness involves "an experience qualitatively similar to that later consciousness which every one agrees to call self-consciousness." (A Reconciliation between Structural and Functional Psychology, in the Psych. Rev., Vol. XIII, No. 2 [March 1906]).

- James Ward, On the Definition of Psychology, in the British Journal of Psychology, Vol. I, No. 1 (Jan. 1904). 


\section{Personality, Intelligence, Consciousness 321}

and Royce have in mind a much more elementary form of self-consciousness.

It goes without saying that the meaning of words is determined by usage, and it is true to-day and probably will remain so for some time that consciousness has the wider and therefore vaguer meaning, even though it would be preferable for the reasons suggested above to limit it to the sense of personality. I have not the space to discuss the conception of consciousness further, but hope that the preceding discussion gives some indication how consciousness influences behavior. I can only repeat that consciousness is a complex process made up of feelings and ideas which are unified by the sense of personality which may begin as a vague feeling, but which becomes in course of time a clear-cut idea.

\section{NATURE OF Mind}

We have now discussed the nature of intelligence and of consciousness and are prepared to take up the subject of the nature of mind. There have been many theories as to the nature of mind, so that it would be impossible to discuss all of them here. I will speak of two of them which have been widely held in the past and which are still held by many. The first is the theory of psycho-physical parallelism. This theory, in the words of one of its adherents, "regards the processes of the material universe (including those of the physical organism) as a closed chain of cause and effect, which is altogether removed from any psychical influence. Mental process is a concomitant of certain highly complex material processes, but not anything that affects these processes themselves." 1 That is to say, ap-

1 E. B. Titchener, Were the Earliest Organic Movements Conscious or Unconscious, in the Pop. Sci. Mo., Vol. LX, p. $45^{8}$ (March, 1902). 
parently, according to this theory, mind is something super. phenomenal, if not supernatural, which for some unaccountable reason always accompanies certain material processes without, however, affecting these processes. The second theory regards mind as capable of causal interaction with body. ${ }^{1}$ That is to say, it assumes, like the parallelist theory, that mind accompanies certain bodily processes, but differs from it in assuming that mind influences these bodily processes and is influenced by them. It is evident that both of these theories regard mind as being a distinct entity of a superphenomenal if not supernatural sort. It must also be evident that science cannot recognize anything of this sort of which it can have no tangible evidence. To assume the existence of such an entity cannot be called a scientific theory, but the most speculative sort of an hypothesis.

What, then, is mind? It is quite likely that we should be better off if the word could be abolished from the literature of the sciences which study the attributes of animals because of the difficulty of assigning to it a definite meaning. But since the term is now firmly intrenched in general use, it is incumbent upon one who is trying to describe the factors which determine behavior to limit its scope and meaning as precisely as possible.

It seems to me that mind is simply a general name for the phenomena which we have been studying under the heads of intelligence and consciousness. If this is correct, then it is evident that mental processes are determined by minute and refined physiological processes which take place in certain parts of the central nervous system. We may then speak of

1 For an elaborate defense of psycho-physical interactionism, see William McDougall, Body and Mind, A History and a Defense of Animism, New York, IgrI. 


\section{Personality, Intelligence, Consciousness 323}

mind as a stage in the determination of certain kinds of behavior. It is the stage between the reception of the stimulus through a sense organ or otherwise and the discharge of an impulse to a motor organ which causes an external form of behavior. This stage manifests itself to the person experiencing it in the form of images, ideas, feelings, emotions, etc., while its presence is made known to the observer by means of certain kinds of variations in behavior. As we have seen, this stage may have great influence upon the behavior which is to result, so as to change completely the character of this behavior.

I believe that the preceding discussion explains satisfactorily the so-called "power of mind over matter" and the alleged superiority of mind over matter which are frequently regarded as very mysterious characteristics of mind. It is the quantitative disproportion between the apparent cause and the apparent result in these cases of "the power of mind over matter" which has led to the belief that mind is immensely superior in quality to matter and entirely different from it. It is therefore believed that a causo-mechanical explanation of mental and social phenomena is impossible. But the preceding discussion has shown that there is nothing mysterious about this power, but that similar phenomena take place constantly in nature whenever a small amount of molecular or molar force releases a large amount of molecular or molar force. We see this whenever a small amount of force in the form of heat is applied to a combustible such as dynamite and results in the dissipation of a large amount of force. It has even been suggested that a process of radio-active change started in the elements might result in the disintegration of the 
earth. "Professor Rutherford has playfully suggested to the writer the disquieting idea that, could a proper detonator be discovered, an explosive wave of atomic disintegration might be started through all matter which would transmute the whole mass of the globe and leave but a wrack of helium behind." 1

The mind, therefore, works like the trigger of a gun or a button which releases an electric current which explodes a mass of nitroglycerin. The amount of energy involved in the mental process is entirely disproportionate to the amount involved in the behavior which it influences and may even control. But this does not prove that it is absolutely different qualitatively from other material processes.

Various attempts have been made to devise a criterion which will indicate whether or not mind is present. For example, Royce ${ }^{2}$ enumerates as the "physical signs of the presence of mind" discriminating sensitiveness, docility, by which he means the capacity shown by an animal in its acts "to adjust these acts not merely to a present situation, but to the relation between this present situation and what has occurred in the former life of this organism," and mental initiative. Yerkes ${ }^{3}$ has improved upon Royce by recognizing structural criteria as well. He enumerates as structural criteria of mind organization, neural-organization, and neural-specialization, and as functional criteria discrimination, docility, and initiative. I think, however, that this chapter has shown that there can be no criterion or criteria of mind apart from the criteria of intelligence and conscious-

\footnotetext{
1 W. C. D. Whetham, The Recent Development of Physical Science, Philadelphia, 1904, p. 243.

2 Op. cil., chap. II.

3 Op. cit.
} 


\section{Personality, Intelligence, Consciousness 325}

ness which I have described, and reference to these criteria will show that they include all that Royce and Yerkes include in their criteria of mind.

I have now completed this discussion of intelligence and consciousness and of the nature of mind in general. It may seem that the conception of mind presented is very vague, but $I$ think that it must be evident by this time that the phenomena grouped under this term are very diverse.

Before closing this chapter I wish to discuss briefly the question as to whether our knowledge of mind is purely subjective or partly objective. Many psychologists believe that it is purely subjective, thus placing psychology upon an entirely introspective basis. Looked at from one point of view, this may seem to be true. It is true that sensations, ideas, feelings, etc., as such, are experienced only by the subject. But the behavior which results from them may be observed by another, and, as we have seen, the character of these psychic phenomena has to be described in large part in terms of behavior. Furthermore, scientific research has shown that these phenomena are determined by neural processes which may be observed. The aspects which psychic phenomena manifest to the subject, on the one hand, and to the outside observer, on the other hand, have been described by Montague in the following words: "What I, from within, would call my sensations are neither more nor less than what you, from without, would describe as the forms of potential energy to which the kinetic energies of neural stimuli would necessarily give rise in passing through my brain." ${ }^{1}$ The fact

1 William P. Montague, Consciousness as Energy, in Essays in Philosophy and Psychology in Honor of William James, New York, Igo8. 
that some observers have been able to introspect their mental processes more effectively because of their knowledge of these neural processes indicates that there is an objective as well as a subjective source of knowledge regarding mind. To deny the possibility of such objective knowledge is, as has been pointed out by several writers, to deny the possibility of a comparative psychology, and we have seen how important this branch of psychology is for the reconstruction of mental and social evolution. 


\section{CHAPTER XVII}

\section{THE BEGINNINGS OF SOCLAL EVOLUTION}

Definitions of association and society, 327. - Colonial species, 328. - Is there an inborn associative tendency? 329. - The external, environmental forces for association, 330. - The degree of correlation between organic and social evolution, 33r. - Internal forces for association, 334. - Inborn and acquired characteristics, 335 .

So far I have been discussing the behavior of living beings in the main as if they were isolated from each other. But the great majority of animals live intermittently or permanently in association with other animals, and their behavior is more or less affected by such association. So that in any comprehensive survey of the forces that determine behavior it is necessary to discuss-association. In the following chapters I shall describe the fundamental types of association and the earlier forms of association among men, making some attempt to explain their causes.

In the discussion of this subject, as in the discussion of so many others, questions of terminology immediately arise. What do we mean by such words as association and society? By association might be meant simply that certain animals are in close proximity to each other. But this would not necessarily involve their affecting each other's behavior so that such association would be of no significance for the determination of behavior. By association is therefore meant usually the sort of proximity which 
results in affecting behavior. But the meaning of the term is frequently limited still further so as to apply only to proximity which affects behavior through mental interaction. By a society is usually meant a group characterized by more or less permanent association. I shall not attempt to define more specifically the technical meanings of these terms at this point, but will turn to the discussion of the ways in which animals come into proximity with each other and the effect which such proximity has upon their behavior.

We have seen that the living cell is the unit in the organic world. Among the protozoa a single cell forms an organism. Metazoan animals are formed of aggregations of cells. Such aggregations are sometimes called associations. Some protozoan species and some of the lower metazoan species form colonies in which the individual members are organically connected with each other. ${ }^{1}$ These connections exist as aids to nutrition. In some of the colonial species, as, for example, the infusoria which form colonies, there is no vascular connection. In other colonial species, as, for example, some of the polyps, mollusks, worms, etc., there is vascular connection which increases considerably the degree of interdependence in the nutritive processes. A certain degree of physiological division of labor develops in some of these colonial species. In fact, in some of these species the relations between the individual members become so close as almost to make each colony a distinct organism. These colonies also are sometimes spoken of as associations, and even as societies. It is evident, however, that if we are to accept the definitions of association and of

\footnotetext{
1 E. Perrier, Colonies Animales, Paris; A. Espinas, Des Societes Animates, Paris, 1878 .
} 
society which have been suggested, these aggregations cannot be regarded as associations or societies, even though a high degree of interdependence may exist among them, because there is no mental interaction. Nevertheless, the study of these aggregations is of importance for social evolution, for it may be that this inborn tendency to unite persists throughout the rest of organic evolution and furnishes the occasion for the mental interaction which develops later.

As yet we know nothing definite about such an inborn associative tendency. Petrucci suggests some of the small amount of evidence which we have with regard to it and some of the possibilities in the following passage: "When we reach the precursors of the vertebrates, the prochordates, we find colonial life, and in the habits of the amphioxus ${ }^{1}$ which joins together several individuals to swim in ribbons there still expresses itself an affirmation of colonial life, a grouping of individuals. Can we go back still further beyond this remote form and claim to find an origin? Then we come to the very constitution of metazoan organisms, to the series of constitutive stages which, by successive differentiations and integrations, have realized the type of the superior organisms upon the basis of associations or colonies of unicellular individuals originally alike. In his work on animal colonies, Edmond Perrier connects with this type of structure the four branches of the ringed worms, the mollusks, the articulates, and the vertebrates. Since the researches of Durand de Gros, it is known how this polyzoic constitution of beings has remained engraved upon their nervous system; it is possible to attach the associative tendency, manifested in so general a way in the animal world as a whole, to these primitive constitutions which have written themselves upon the very structure of the individual and which are projected as psychic acts into its external activity." 2 M. Petrucci goes on to express the opinion that in this "associative tendency" we have a manifestation of a universal rhythmic movement which manifests

1 A low form of vertebrate.

2 R. Petrucci, Origine polyphylétique, homotypie et non-comparabilitê directe des societes animales, Brussels, 1906, pp. I21-122. 
itself also in chemical affinities and in the force of gravity, so that "the associative tendency becomes a peculiar expression of this enormous rhythm which measures the evolution of the universe and which engraves itself, under a peculiar aspect, in the irresistible impulse by which the animal world is guided."

But it is best for the present to treat very critically any such theory of an associative tendency, for there is very little evidence of its existence as yet. In the light of the preceding chapters it must be evident that it must be reduced to the concrete terms of specific forces before we can be sure of its existence. In other words, it must be shown to exist in the form of tropic reactions in the lower animals and of reflexes in the animals with a nervous system. Simply because these animals come together, it does not necessarily follow that it is due to an associative tendency. Certain external forces bring them together. For example, animals living in the water will gravitate towards the area where the temperature is most favorable for their life processes. If this area happens to be small, a large number of the same species may be found in close proximity to each other, and yet have come together quite independently of each other. The pressure of the water may also influence this orientation. Thus it is that large shoals of fish are to be found in certain strata of water which is to be explained in part at any rate by the temperature and the pressure. Furthermore, the location of food determines the orientation of the members of all species to a large extent. If the food of a species is distributed over a small area, the members of the species will be forced to come together in the course of their food quest.

But the members of a species may influence each other 
to come together without any associative tendency being in existence. For example, in discussing the behavior of the lower animals I have mentioned such a case in connection with certain unicellular animals which excrete a certain chemical. If a number of members of one of these species come into close proximity to each other by accident, the water in that region becomes more or less fully charged with this chemical. If, then, these animals react negatively when they strike the boundaries of this chemicated area in the manner described earlier, they will be held in this area in close proximity to each other. Other animals coming into the same area will be held in similar fashion, so that in course of time there may be a large aggregation due to the physiological processes of these animals. But this could hardly be regarded as a manifestation of an associative tendency on the part of these species.

It goes without saying that social phenomena are a part of universal phenomena and that social evolution is a part of the general evolutionary process. But in trying to explain these phenomena it does not help much to postulate an associative tendency the nature of which we are unable to explain. The same is true of the so-called gregarious instinct which is frequently cited in attempting to explain the later stages of social evolution and which we shall discuss later. Therefore, without assuming that an associative tendency has necessarily been at work throughout organic evolution, for some would extend this tendency to the vegetable as well as to the animal world, let us discuss briefly the probable causes for social evolution.

The phenomena of association have displayed themselves at many points in organic evolution and among many 


\section{The Science of Human Behavior}

species. Is it possible to arrange these phenomena in their evolutionary order so that each group will have evolved directly from the group which precedes it? I think it is evident that this can be so for social phenomena no more than it is for organic phenomena. There have been many divergent lines of social evolution just as there have been many divergent lines of organic evolution. Along some of these divergent lines social evolution has proceeded very far, quite independent of other lines of evolution. So far as social evolution has been due to hereditary causes, the divergence must always have been quite as great as the divergence in the organic series. So far as social evolution has been due to other than hereditary forces, the degree of divergence has depended upon conditions which will be discussed later. This does not mean, however, that each divergent line if traced back far enough does not come to a common source with every other line. It is also possible to arrange groups of social phenomena in series according to their degree of complexity or according to any other standard, even though evolution has not actually taken place according to that series.

If, therefore, we are to assume that human social phenomena are of the highest type, it does not follow that all lower types have been in the same evolutionary series. In order to determine the series for man, it is necessary to determine to what organic series he belongs, and the series for social phenomena will correspond in large part, if not entirely, to the organic series.

M. Petrucci in his suggestive monograph on the relations between animal societies which has already been cited discusses this point so well that I cannot do better than to quote from him: "We do not 


\section{The Beginnings of Social Evolution}

know if the vertebrates are descended from the articulates or from another branch of invertebrates; however, each of these branches presents in its classes, its orders and its genera phenomena of a social order. The same is true for the prochordates which are most closely related to the ancient type which was the origin of the vertebrates. In the group of the selacians, in which is to be found the existing example most closely related to the type of fish which could have led to the continental vertebrates, are to be found social characteristics. But it is evident that the divergence of the original stock of the vertebrates from that of the existing prochordates, which has separated all the fishes of the transitional type leading to the continental vertebrates, is very ancient and has not left any precise trace. It is by analogy only that we could reconstruct an inherited social character. The separation of the vertebrates from the invertebrates has already taken place in Silurian time, so that it must have happened in Cambrian time, and since that moment there is no more possibility of a direct comparison between animal societies of invertebrates and of vertebrates. In the same fashion, the divergent evolution which has separated the fishes from the reptiles began as early as Silurian time, and since that time there is no more possibility of inheritance in a direct line and of a direct comparison between societies formed by fishes and those formed by reptiles. The divergence which has separated from a common source the three classes of reptiles, mammals, and birds must have taken place no later than the end of Permian time during the Primary period, because at the beginning of the Secondary period are found mammals, reptiles, and traces of birds. From that time the direct comparison of societies formed by these three classes is no longer justified. In the next place, within the mammals themselves the orders of monotremes and of marsupials, differentiated from a common source, can no longer be compared directly. The other orders of mammals, the rodents, carnivora, and herbivora, differentiated from a type which they share with man, have been able to inherit a common social tendency, but have varied from it in different ways which destroy all possibility of direct comparison. In the last place, in the direct line to man the lemurs are in the same position as the marsupials and monotremes, and it is only with the apes belonging to the order of primates, that is to say, with the anthropoids, that societies formed by man could be most narrowly compared. However, the divergence, at this point also, 
having displayed itself from the starting point furnished by a primate previous to the pithecanthropus ape, direct comparison is no more possible. We arrive, therefore, at this conclusion: the polyphyletic origin and the direct non-comparability of animal societies." 1

However, even though human society is not in the same evolutionary series with any other animal society now in existence, it is still worth while to study these other societies, and such study will throw light upon the evolution of human society. This is true, in the first place, because while these species, including the human species, may not have inherited in common an associative tendency, they have inherited in common certain structural forms and other characteristics which have deternimed in part and have conditioned the social phenomena displayed by all these species. In the second place, all these species have been subjected to a certain extent to the same conditions, so that their social characteristics are in part due to similar environmental conditions. So it is that similar structural. forms and functions, such as those of nutrition, reproduction, etc., on the one hand, and similar environmental conditions, such as topography, climate, distribution of food; etc., have produced along divergent lines of organic evolution social phenomena which are to a certain extent similar. As we shall see, the same is true of mental phenomena, and to study either the mental or the social phenomena of any species is to throw light upon the corresponding phenomena of any species possessing them. The preceding statements will explain why this chapter is entitled the beginnings of social evolution, for social evolution, like mental evolution, has had many beginnings along widely divergent lines of or- 
ganic evolution, and yet there have been many analogies and likenesses between these different lines of social evolution.

I have already spoken of the way in which the food quest brings together members of the same species when their food is distributed over a small area. As organic evolution progresses, the reproductive needs play a more and more important part as a cause of association. We have seen in an earlier chapter that at the beginning of organic evolution organisms were in large part, if not entirely, independent in their power to reproduce. That is to say, in the case of most, if not all, individuals reproduction could take place simply by fission. But comparatively early, if not from the very start, conjugation became necessary between certain individuals. That is to say, it became necessary for independent lines of descent to cross occasionally, or else they would lose the power of reproduction. Conjugation in these low species probably takes place usually as the result of chemotropic attraction. Here, then, was a force bringing members of the same species into contact with each other. Then gradually, as we have seen, among the lower metazoan species began to arise the anatomical and physiological differentiation between the two sexes. When the physiological division of labor in reproduction based upon this differentiation became firmly established, coöperation between the two sexes became almost invariably necessary for reproductive purposes. In the higher species there developed a combination of reflexes which we call the sexual instinct and which has ever since been a cause for association along many divergent lines of evolution. In this instinct we find a common heritage of all species that have started on a line of social evolution. 
But as organic evolution continued, more than the sexual instinct became necessary for reproduction to be effected successfully. As animal forms developed and became more complex, more time was needed for the development of the individual animal from the beginning of its life to its full development. During a part, at least, of the time needed for attaining maturity the animal was helpless and in need of care. Thus there developed the parental instincts leading one or both parents to care for the young during this period of helplessness. Where the care is given by one parent, more usually it is the female, though sometimes it is the male, as in the case of many fishes and certain birds. From parental care there results association between parents and their young which, when it becomes more or less permanent and stable, is called the family. In these instincts we again find a common heritage of most, if not all, species that have started on a line of social evolution.

As the nervous system, and especially the central nervous system, evolved, the anatomical basis for the development of intelligence was furnished, as we have seen in the last chapter. The differentiation of the nerve cells from the other somatic cells began very early among metazoan species, but the development of the nervous system has taken place along many divergent lines of evolution. Wherever the nervous system has developed in such a fashion as to make association possible, intelligence has become possible and has therefore made its appearance along many divergent lines. Wherever it has made its appearance and has developed to any extent, it has become a powerful force for association in various ways. It has made members of a species susceptible to suggestion from members of the same 
species and has thus led them to imitate. It has enabled members of a species to consciously recognize other members of the same species as such. It has enabled members of the same species to communicate with each other and has thus enabled them to conserve ideas, etc., by transmitting them from one generation to another in the form of tradition. In the anatomical basis of intelligence, then, we find still another common heritage of most, if not all, species that have started on a line of social evolution.

I have now indicated very briefly the different types of forces which give rise to association; namely, certain characteristics of the environment, such as the distribution of food, temperature, climate, etc., certain inborn characteristics, such as the sexual and parental instincts, and certain acquired characteristics comprised under the head of intelligence, which is made possible by an inherited anatomical structure. There is not enough space in the remaining chapters to present all the data with respect to the beginnings and early stages of social evolution along the many divergent lines of evolution, even though these data are still very limited in comparison with the number and difficulty of the problems involved. Some of these data are paleontological and furnish evidence, in the form of fossils distributed in small areas, of association on the part of extinct species. But most of these data are with respect to living species representing many divergent lines of evolution. I shall therefore only discuss briefly the data with respect to a few species representing different parts of the animal kingdom with the hope that such discussion will throw some light upon the causes for the beginnings of social evolution and the forces which determined the character of the early stages of this evolution. 


\section{CHAPTER XVIII}

\section{INSECT SOCIETIES : THE ANTS}

Anatomical polymorphism, 339.-Physiological division of labor, 339. - The philoprogenitive instincts, 342. - The founding of an ant community, 343. - The food-procuring activities, 345.-The hunting stage, 346 . - The agricultural stage, 347. - Harvesting, 347 . - The pastoral stage, 347.-Symbiosis, 347.-Parasitism, 351.-Myrmecophilism, 351. - Commensalism, 351. - Slave making, 357.-The intelligence of ants, 359. - The ant brain, 359. - Recognition, 360. - Communication, 362. - Suggestion and imitation, 362. - Coöperation, 363. - Insect societies are based mainly upon instincts, 363 .

Among the invertebrates the insects have gone furthest along the road of social as well as mental evolution, as illustrated by the ants, bees, social wasps, etc. I shall therefore discuss briefly the social phenomena of the ants, since they display what is probably the greatest range of such phenomena among the insects.

It is well known that ants live in large communities numbering sometimes, it has been estimated, hundreds of thousands of individuals. Their nests, in the form of hills, subterranean passages, etc., require in their construction a high degree of concerted action. It may appear, therefore, that they must be endowed with a high degree of intelligence, and this has been the opinion of many people in the past. But a more careful examination of the ants and their behavior shows that most of these social activities can be explained without the aid of intelligence. 


\section{POLYMORPHISM}

The first thing to be noted is the morphological or anatomical polymorphism which characterizes the ants. All species above the asexual stage are characterized by sexual dimorphism. But there are certain species in which one or both of the sexes appear under two or more distinct forms. Such polymorphism is to be found in what is perhaps its most fully developed form in the social insects, such as the termites (which are sometimes called the white ants, though they are not at all related to the ants), in which both sexes are equally. polymorphic, and the ants, social bees, and wasps, in which usually the female alone is differentiated into distinct castes. Sexual dimorphism makes possible a division of labor so far as reproduction is concerned. Polymorphism permits of a much more extended division of labor, which is frequently similar to the division of labor which characterizes human society. But human division of labor is largely intelligent in its origin, while division of labor based upon polymorphism is not necessarily accompanied by intelligence. Such division of labor is termed physiological and is due to congenital differences of structure, which necessarily involve different modes of behavior.

The question of the origin of polymorphism is an important one in this connection, but I shall discuss it only very briefly, in the first place, because it is still unsettled, but more particularly for lack of space. Wheeler in his valuable discussion of this subject suggests that it may have resulted from a physiological division of labor, in the following words: "Polymorphism is of rare occurrence in the 
animal kingdom and may be said to occur only in colonial or social species where its existence is commonly attributed to a physiological division of labor." 1 And again he speaks more particularly of its origin in the group of insects to which the ants belong. "This restriction of polymorphism to the female in the social Hymenoptera, with which we are here especially concerned, is easily intelligible if it be traceable, as is usually supposed, to a physiological division of labor, for the colonies of ants, bees, and wasps are essentially more or less permanent families of females, the male representing merely a fertilizing agency temporarily intruding itself on the activities of the community at the moment it becomes necessary to start other colonies. We may say, therefore, that polymorphism among social Hymenoptera is a physical expression of the high degree of social plasticity and efficiency of the female sex among these insects. This is shown more specifically in two characteristics of the female, namely, the extraordinary intricacy and amplitude of her instincts, which are thoroughly representative of the species, and her ability to reproduce parthenogenetically." 2 It is, however, difficult to believe that polymorphism could have resulted from a physiological division of labor, for this would be putting the function before the structure it characterizes. In fact, it seems quite necessary that polymorphism should exist before a physiological division of labor could be possible. It seems more probable that polymorphism must be due either to determinants in the germ plasm which stand for the different castes or differences in the conditions under which the

\footnotetext{
1 W. M. Wheeler, Ants, their Structure, Development and Behavior, New York, I9I0, pp. 86-87.

2 Op. cit., p. 87 .
} 
young develop. Both of these theories have been proposed and discussed. For example, Weismann advocated the germ plasm theory, as might be expected. Many of those who have held the other theory have believed that the differences between the different castes are due to differences in nutrition; that is to say, differences either in the quantity or quality of the food given to the young. This last theory seems to be the most plausible one, but nothing certain is as yet known, as is indicated in the following passage: "We may conclude, therefore, that while the conception of the worker phase as the result of imperfect nutrition is supported by a considerable volume of evidence, we are still unable to understand how this result can take on so highly adaptive a character. Such a concise effect can hardly be due to manifold and fluctuating external causes like nutrition, but must proceed from some more deeply seated cause within the organism itself. Of course, the difficulty here encountered is by no means peculiar to polymorphism; it confronts us at every turn as the allpervading enigma of living matter." 1

However, whatever may have been the origin of polymorphism, the important part it plays in the social life of ants is very evident. The division of labor based upon it causes a high degree of interdependence within the ant community. The polymorphism exists principally within the third or worker caste, which are females which are sterile, apparently owing to the lack of food. The males are few in number and are usually very stable. The females are relatively stable, but less so than the males. The workers are very unstable and appear in many species in many differ-

${ }^{1}$ Wheeler, op. cit., p. 109. 
ent forms, each being fitted by structure to perform a peculiar function.

\section{Philoprogenttive Instincts}

The part played by the philoprogenitive instincts in the social life of the ants must be noted next. In fact, it is probable that these instincts have formed the strongest force for social evolution among the ants. Wheeler indicates their importance in the following words: "In the lives of the social insects the threptic or philoprogenitive instincts are of such transcendent importance that all the other instincts of the species, including, of course, those of alimentation and nest building, become merely tributary or ancillary. In ants, especially, the instincts relating to the nurture of the young bear the aspect of a dominating obsession. Their very strength and scope render the insects more susceptible to the inroads of a host of guests, commensals and parasites." 1 It is difficult to explain why the philoprogenitive instincts are so strong among the ants. The ancestors of the ants were solitary in their habits. They must have developed philoprogenitive instincts to a certain extent, otherwise their offspring would not have survived. But some of their descendants have developed these instincts much less than the ants, as, for example, the wasps, which do not see their offspring at all, having buried the egg with a caterpillar or other animal as food for the larva to feed upon after it has hatched. The ants, on the contrary, care for the young for a long time after hatching and in many different ways. This may be due to the fact that the young of the ants pass through four 
stages before they mature. These stages or instars are known as the egg or embryo, the larva, the semipupa or pseudonymph, and the pupa or nymph. This high degree of complexity in the ontogenetic development of the ant probably explains in part the polymorphism of ants, for by lack of nutrition or in some other way the development of the ant can be arrested at certain points. As each of these stages needs special care of its own, the philoprogenitive instincts have to be developed very greatly for the preservation of the species.

Let us trace very briefly the founding of an ant community. A female, having been fertilized at the time of the nuptial flight, becomes dealated by rubbing off its wings. It then seeks for a convenient spot for founding its nest in a protected place. Having found it, perhaps under a large stone, it shuts itself in from the external world, and in course of time begins to lay eggs. During all the period of egg laying it does not go out for food, but lives off the nutritive matter which is stored up in its wing muscles. When the first eggs are laid, the female, or queen as it is usually called, cares for them and the young which are hatched from them until they mature. So far the group constitutes no more than a family. But now is manifested the phenomenon which is to make the group far more extensive and complex than a family. The workers which appear are sterile as a rule, as has been indicated. But they have apparently inherited some philoprogenitive instincts from their mother, for instead of leaving the nest to live by themselves or to found a new nest, they go out to forage for food, which they bring back to the nest and feed to the brood and to the queen. In many other ways they manifest these instincts 
by caring for the brood. Thus we have the singular spectacle of these worker ants, thwarted from breeding themselves because of the arrested development of their reproductive organs, lavishing a parental care upon their fellow offspring. Held, therefore, to the maternal nest by these philoprogenitive instincts and enabled by the polymorphism which characterizes every species of ants in varying degrees to differentiate their activities, the family group founded by the queen develops into a vast community carrying on extended social activities.

The importance of polymorphism and the philoprogenitive instincts for the evolution of ant society has been well stated by Wheeler in the following passage: "Owing to this preëstablished structure and the specialized functions which it implies, ants are able to live in a condition of anarchistic socialism, each individual instinctively fulfilling the demands of social life without 'guide, overseer, or ruler,' as Solomon correctly observed, but not without the imitation and suggestion involved in an appreciation of the activities of its fellows. An ant society, therefore, may be regarded as little more than an expanded family, the members of which coöperate for the purpose of still further expanding the family and detaching portions of itself to found other families of the same kind. There is thus a striking analogy, which has not escaped the philosophical biologist, between the ant colony and the cell colony which constitutes the body of a Metazoan animal; and many of the laws that control the cellular origin, development, growth, reproduction, and decay of the individual Metazoan, are seen to hold good also of the ant society regarded as an individual of a higher order. As in the case of the individual animal, no further purpose of the colony can be detected than that of maintaining itself in the face of a constantly changing environment till it is able to reproduce other colonies of a like constitution." 1 


\section{FOOD-PROCURING ACTIVITIES}

The part played by intelligence in the social life of the ants will be discussed later. I wish now to speak of the part played by external forces. Inasmuch as ants live on land and are inured to varying conditions, they are not restricted to narrow limits of temperature and of atmospheric pressure. But the distribution of their food supply is for them, as for every species, an important factor in determining the extent to which their life is to be social. Species whose food is scanty and scattered are forced to live more or less solitary lives. Predatory animals, also, such as the mammals, birds, and insects of prey, are likely to be solitary in their habits, because hunting can usually be carried on best alone. Vegetarian species, on the other hand, are likely to be social, because their food is usually abundant and is more or less concentrated in places where conditions are favorable for its growth. The family of the Formicidæ which the ants constitute was formerly carnivorous. Of the five subfamilies of this family only the two lower, the Ponerinæ and the Dorylinæ, are now purely carnivorous. And it is a significant fact that these subfamilies have less permanent and complex societies than the other three. The colonies of the Ponerinæ are rare and of small size. The colonies of the Dorylinæ, though sometimes very large, are of a nomadic character, since they must always be seeking new hunting grounds in order to secure enough food. The ants of the three higher subfamilies, the Myrmicinæ, the Dolichoderinæ, and the Camponotinæ, though sometimes predatory, have become adapted to a more varied diet, and some of them live almost entirely on vegetable 
food. These subfamilies have developed the most stable and complex societies among the ants. ${ }^{1}$

The food-procuring activities of the ants present a series of very interesting and significant phenomena which have been interpreted by some as indicating a high degree of intelligence on the part of the ants. But it is probable that these activities can be explained in the main on other than intelligent grounds, though intelligence may play a small part in them. I will describe briefly some of the principal activities.

Three economic stages have been distinguished among the ants corresponding to similar stages among men. These are the hunting, pastoral, and agricultural stages. The activities which characterize each of these stages have been described by some observers in such a way as to seem to imply that they involve nearly, if not quite, as much intelligence as among men. But a more careful examination of the data seems to indicate that they involve much less intelligence than among men. The lowest stage among the ants, as among men, is the hunting stage. It has already been indicated that the two lower subfamilies of the Formicidæ are the hunting ants and that these are less developed socially than the other ants. The tendency to hunt is largely an instinctive matter among the ants, as it is among all animals. That the hunting of ants involves much less intelligence than the hunting of men is indicated by the fact that ants do not use weapons, though this difference may be accounted for by differences in structure.

Among men the pastoral stage usually succeeds the hunting stage. But among the ants the pastoral stage involves 1 Cf. W. M. Wheeler, op. cit., pp. I76-177. 
more complex phenomena than the agricultural stage and seems to display the exercise of more intelligence, so that $I$ will discuss the agricultural stage first. The agricultural stage is exhibited by the so-called harvesting ants, which store seeds in granaries in their nests. This practice is probably largely the result of instincts which may be closely related to the philoprogenitive instincts. Food which is not needed immediately is set aside to be used later in feeding the brood. But some observers have claimed that certain species not only store away food in this fashion but also plant seeds and cultivate the crop which grows from them. If this were true, it would be difficult to explain it solely on the basis of instinct and would seem to involve a degree of intelligence almost equal to human intelligence. But it seems quite clear that this is not the case. The observers who have claimed this have based their assertion on the fact that some of the hills of the harvesting ants are surrounded by a ring of the kind of plants from which they harvest their seeds. But the presence of this ring is in all probability purely accidental so far as the ants are concerned. It is probably due to seeds that have been dropped by ants on their way to the nest and to seeds in the chaff which has been rejected. Some of these seeds sprout and take root and thus in course of time a field of these plants grows up around the hill. ${ }^{1}$

\section{Symbiosis}

Let us now turn to the so-called pastoral stage as exhibited by the ants. This brings us to a subject which I wish to discuss in this connection, namely, that of associa-

${ }^{1}$ Cf. W. M. Wheeler, op. cit., pp. 286-287. 
tion between members of different species. So far I have been discussing only association between members of the same species or at any rate between members of closely related species. But there are various kinds of association between animals which are more or less different, and these kinds of association must be considered in studying the origin and evolution of society, for they have played some part in social evolution. Relations of a symbiotic character also exist between animals and plants which are of great importance for both. Such relations exist in the case of certain species of ants. But inasmuch as plants have no mental characteristics, such relations can never become social in the strict sense of the term. Mental interaction can, however, exist between members of different animal species, and social relations can therefore become established between them. It goes without saying that the extent to which such mental interaction can exist and such social relations can be established varies more or less directly with the degree of difference between species. The mental interaction may take place in the form of suggestion and imitation. Parental care may be lavished by an adult of one species upon the young of another species, thus establishing a social relationship similar to that between parents and offspring. This is not surprising when the species are more or less alike, as when a hen adopts some ducklings, but it is surprising when they are very different, as in the case reported by Lloyd Morgan where a hen adopted some young ferrets. Such a thing can happen, of course, only when parental feeling is strong in the adult so that it is prone to give vent to it by caring for the first young and helpless of any species which it happens to meet. We shall see a little 
further on that ants misdirect their philoprogenitive instincts in this fashion sometimes.

The term pastoral when used among men is applied to the activities of man in caring for animals in flocks or herds which have been domesticated by him and which he is going to use usually as food or from which he is going to procure food. Let us see what activities among the ants correspond to such pastoral activities among men. Certain species of ants secure much of their food from certain insects which secrete juices from plants, which they excrete in the form of a liquid like honey and which the ants like very much. Among these insects are the plant lice (Aphididæ), scale insects or mealy bugs (Coccidæ), tree hoppers (Membracidæ), lantern flies (Fulgoridæ), jumping plant lice (Psylli$\mathrm{dæ}$ ), and the caterpillars of the Lycænid butterflies. The symbiotic relations which become established between the ants and these insects are most evident in the case of the aphids, so I will speak of them. The aphids excrete their honey from the anal orifice in the form of drops. The ants caress them with their antennæ, and this stimulates them to evacuate the honey. The aphids seem to like the caressing, so that there becomes established a symbiotic relationship which is mutually profitable to both kinds of insects. But the aphids profit in still other ways from their relations with the ants. The ants protect them from insects which prey upon them. These insects, of course, seek the aphids for a reason similar to that of the ants, namely, to secure food. But many of them would kill the aphids if they succeeded in reaching them, instead of simply taking from them an edible product, as the ants do. So that the protection of the ants is very useful to the aphids. Then many of the aphi- 
dicolous ants construct small inclosed pavilions, or sheds, which serve for the protection of the aphids as well as of themselves. Many observers have thought that these sheds are built for the express purpose of housing the aphids; but it is more likely that they are constructed as the result of an instinct which leads them to excavate tunnels and chambers around roots, and then the aphids are brought there because the ants can feed from them more conveniently there. So that the protection afforded the aphids by these constructions is an accidental and unintentional result. Aphidicolous ants also sometimes collect and store aphid eggs in their nests, bringing up the young aphids after they have hatched. This has seemed to some observers a purposeful raising of these aphids in order to secure food from them after they have matured. But it is quite probable that this is a case of misdirected parental care, such as I have already discussed.

It is sometimes said that the aphids are the cows of the ants and it is true that there is something of an analogy between the relation of aphids to ants and that of cows to men. But it is an analogy that should not be carried too far, as I believe the above considerations show. Cattle were originally wild and had to be tamed and domesticated by man before he could make use of them for securing food in the form of milk. But the aphids seem to be very docile creatures, and it is very doubtful if the ants ever had to domesticate them. It goes without saying that intelligence does not have to be assumed to explain why the ants eat the honey which falls from the aphids. The caressing of the aphids in order to induce them to evacuate the liquid may be an acquired habit and may indicate a small amount 
of intelligence, a connection in the associative areas having become established between the act of caressing and the securing of food. But even this practice of the ants may be due to some obscure instinctive tendency which we do not understand. As we have seen, the constructing of sheds and the bringing up of the young of the aphids are probably due to instincts with which we are familiar. So that it seems quite evident that the analogy between the pastoral stage among men and the so-called pastoral stage among the ants is in the main a superficial one and is principally an analogy between external appearances. However, this so-called pastoral stage furnishes a highly interesting example of a symbiotic relationship between widely different species from which both benefit and which is therefore worthy of study.

In their relations with the aphids and the other insects mentioned above the ants assume an active rôle and are to a certain extent parasitic upon them. But the ants have certain other symbiotic relations with other animals in which the ants are usually either passive or indifferent. In these relations the other animals force themselves upon the ants and become either parasites upon them or commensals with them. These animals when they inhabit ant nests either throughout life or during one or more of their developmental stages are called myrmecophiles or ant guests. Inasmuch as myrmecophilism illustrates parasitism and commensalism I shall describe it briefly.

Wasmann and Escherich have estimated that there are over three thousand myrmecophilous species, including a large number of Arthropoda, of which more than a thousand are beetles, a certain number of Arachnida, and a few 
Crustacea. Wasmann, who has studied myrmecophilism more than any other investigator, has classified the myrmecophiles as follows:-

I. Inimically persecuted intruders, or synechthrans.

2. Indifferently tolerated guests, or synœketes.

3. True guests, symphiles, or myrmecoxenes.

4. Ecto- and entoparasites.

The fact that there are so many myrmecophilous species indicates that ant nests must be attractive to small animals. Wheeler gives several reasons for this attractiveness. "In the first place, the nests are usually permanent abodes inhabited for months or years by successive broods of ants. Second, these nests have at all seasons a slightly higher temperature than the surrounding soil. Third, there is usually more or less refuse food or offal, pupal exuviæ, and dead ants, at least in the superficial chambers. Fourth, the living larvæ and pupæ represent an abundant and highly nutritious food supply for any insects that can elude the watchfulness of the ants. Fifth, the ants, in protecting themselves from larger animals, necessarily protect any small organisms living in their nests. Sixth, the philoprogenitive instincts of the ants are capable of being deceived and exploited, for these insects are so fond of nursing that they are always ready to lavish their affections on any organisms that resemble ant larvæ. Since the dwellings of termites, social wasps, and bees offer many of the attractions here enumerated, it is not surprising to find that these insects, too, have their nest mates and parasites." 1

The first class of myrmecophiles mentioned above, the synechthrans, are not very numerous. They live in ant 1 Op. cit., pp. 379-380. 
nests as scavengers and kill isolated ants. The ants drive them from the nest whenever possible.

The second class of myrmecophiles, the synœketes, live in the ant nest either because they are not noticed by the ants or because they do not arouse great animosity. They are not noticed by the ants when they are too small, too colorless, or too slow of movement to be perceived, or when they have no specific odor which distinguishes them from their environment. In the case of some of the synœketes the ants cannot seize and hold them because of their anatomical structure and therefore cannot remove them from the nest. Some synœketes pay no attention to the ants or their brood, seeking only the refuse in the nest, and are therefore left unmolested by the ants. Some of them develop mimetic characteristics which make them resemble the ants themselves or symphiles. Some synœketes perform services for the ants as, for example, strigilating them. But these synœketes are very nearly if not quite symphiles, which I shall discuss next.

The symphiles, or true guests, which are very largely beetles, live on amicable terms with the ants in their nests. The ants lick them and feed them and frequently care for their eggs. The ants lick the symphiles apparently because they are very fond of the secretion which oozes from these creatures. The symphiles stroke the ants with their antennæ, and this seems to stimulate them to regurgitate food, perhaps because they mistake this stroking for similar treatment from their fellow ants, and this stroking stimulates the instinctive tendency to regurgitate. The caring for the eggs of the symphiles by the ants is apparently an expression of their philoprogenitive instincts. "The philo- 
progenitive instincts are quite sufficient to account for the phenomenon, which is merely a parasitic disease, or instinct aberration, comparable to the rearing of the young cuckoo by its foster parents, or the rearing of puppies by cats, of kittens by hens, etc. In these cases we do not postulate a special hereditary instinct modification, but a simulation of the normal stimulus by an abnormal object. The instinct action is normal, but adapts itself to the changed conditions, and there is nothing to indicate that the instinct disposition has undergone any philogenetic change." 1 The caring for the symphilic eggs is indeed to the detriment of the ants, for the larvæ of the symphiles and indeed the symphiles themselves frequently eat the ant brood, and it is only the great reproductive powers of the ants which preserve them from extinction. This indicates that the caring for the symphilic eggs and young by the ants is the result of a blind instinctive tendency, not the result of intelligence. Further indication of this is that the ants, after burying the symphilic larvæ as they bury their own larvæ, dig up as many of them as they find at the time of pupation. But while this is the right thing for their own pupæ, it usually results in the death of the symphilic pupæ. So that the caring for the symphilic young by the ants results in injury to the symphiles in some cases, when these young are killed off, and when they survive it may result in injury to the ants themselves because the symphiles may eat the ant brood.

The ectoparasites and the entoparasites are still more dependent upon their hosts than the symphiles. The ectoparasites are small creatures that live on the outside 1 W. M. Wheeler, op. cit., p. 4 Ir. 
of the ants, feeding off of them in various ways. The entoparasites are either still smaller creatures which live inside of the ants or they are the larvæ of flies and other animals which are hatched inside-the ants, from which they emerge later, sometimes not before they have killed their hosts.

These symbiotic relationships of the ants which have just been briefly described vary greatly as to the extent to which they furnish the basis for association in the sociological sense of that term. It is doubtful if there could ever be any mental interaction in a purely parasitic relationship. A thoroughly parasitic species is usually very little developed in many of its parts, and this would be true of the parts which determine the mental characteristics. For the pure parasite the host is probably never anything more than a source of food and of warmth. But in the relations of the ants with the symphiles there is possibility for a certain amount of mental interaction. They may influence each other's behavior to a certain extent by means of suggestion, imitation, etc. The same is probably true to a lesser degree of the relations of the ants with the synœketes and the synechthrans. The same might also be true of the relations of the pastoral ants with the aphids and other species exploited by them, but this is very doubtful because of the very low mental development of the exploited species.

These symbiotic relations displayed by the ants appear in many divisions of the animal world. Parasitism is a very widespread phenomenon. In fact it is quite likely that the majority of living species are either parasites or the hosts of parasites. However, as I have indicated, 


\section{The Science of Human Behavior}

parasitism is not of much significance for association and social evolution. The other symbiotic relations appear less frequently. The highest type of such relationship and the one which is of most significance to us is the relation between man and the animals that he has domesticated. Here we find what is without any doubt the highest degree of mental interaction between different species which has been developed anywhere in the animal world.

There is still another form of symbiosis manifested by the ants which must be mentioned here. This is the symbiosis between ant colonies of different species. It has already been indicated that the ants constitute the family of the Formicidæ, which includes a very large number of species. Under this form of symbiosis are also included usually similar symbiotic relations between ant colonies and colonies of termites. These cases of symbiosis are usually classified under the heads of compound nests and mixed colonies. "Different species of ants or of ants and termites are said to form compound nests when their galleries are merely contiguous or actually interpenetrate and open into one another, although the colonies which inhabit them bring up their respective offspring in different apartments. In mixed colonies, on the other hand, which, in a state of nature, can be formed only by species of ants of close taxonomic affinities, the insects live together in a single nest and bring up their young in common. Although each of these categories comprises a number of dissimilar types of social symbiosis, and although it is possible, under certain circumstances, as will be shown in the sequel, to convert a compound nest into a mixed colony, the distinction is nevertheless fundamental. It must be admitted, 


\section{Insect Societies: the Ants}

however, that both types depend in last analysis on the dependent, adoption-seeking instincts of the queen ant and on the remarkable plasticity which enables allied species and genera to live in very close proximity to one another. By a strange paradox these peculiarities have been produced in the struggle for existence, although this struggle is severer among different species of ants than between ants and other organisms." 1

The symbiotic relations established under this form of symbiosis are very varied. Two or more colonies of different species of ants or of ants and termites may locate their nests in contiguity or in close proximity by chance. If in going and coming they meet frequently, they may influence each other a good deal and thus the symbiotic relation may mean something. If, however, they do not meet very much, the relation may mean very little. If their nests become connected by galleries, they will mingle a good deal and thus influence each other a good deal. Sometimes a nest is located near another nest for the purpose of marauding its inhabitants. There is not the space to describe all the different kinds of relations which develop between different species of ants or between ants and termites. But one very extraordinary practice of certain species must be described briefly. I refer to the so-called slave making carried on by certain species. By this phrase some observers have meant that these ants consciously make slaves just as we have seen it has been thought that the pastoral ants consciously domesticate the aphids and other animals. But we shall see that these slave-making

1 W. M. Wheeler, op. cit., 423-424. 
practices are not carried on for the purpose of making slaves but as the result of certain instincts.

It sometimes happens that a queen ant in search of a nest will enter the nest of another species and will succeed in forcing the workers in this nest to adopt her and to bring up her young. In such a case the queen and her young become temporarily and sometimes permanently parasitic upon the original inhabitants of the nest. But this can hardly be called a case of slave making. As we have already seen, certain species are in the habit of raiding the nests of other species and stealing their young. This is probably due primarily to hunger. But the philoprogenitive instincts inherited from the queen may have something to do with it, for the females sometimes steal eggs from other nests evidently for the purpose of rearing the young which will hatch from them. At any rate some of these eggs and young are usually eaten. Those that are not eaten are reared with the young of their captors and become permanent members of the nest. If this was all, then the capture of these eggs and young would simply have resulted in the formation of a mixed colony. But some of these species have a tendency to become dependent and parasitic upon these alien ants which they have captured. In some cases they become so dependent that they would perish without the care of their so-called slaves. The causes for the development of this parasitism are very obscure. But it seems quite evident that beyond the force used in capturing them there is no compulsion exercised over the alien ants in the nest, so that the term slave is a very inaccurate one to apply to them. 


\section{INTELIIGENCE}

Before closing this discussion of the social behavior of the ants I must discuss briefly their intelligent characteristics. It was stated in the last chapter that it may not be legitimate to speak of association where there is no mental interaction and that no society can evolve without such interaction. Several times in the course of this discussion of the ants it has been suggested that under certain circumstances mental interaction may exist between ants in the form of suggestion, imitation, etc., and also between ants and other animals. It is very difficult to secure data with regard to such mental interaction in the case of animals so small as the ants. It is only by observing variations in their behavior that we can conjecture as to how they are being influenced by others. However, I will summarize some of the evidence with respect to this point.

As we have seen in previous chapters, no variations in behavior which can be called intelligent or conscious can take place unless there are association areas in the brain, and therefore there can be no mental or psychic phenomena without such association areas. If this is true, it is evident that there can be no mental interaction without such association areas. It is therefore necessary to investigate first the nervous system of the ants in order to determine whether they possess the necessary neural basis for mind.

The frontal lobes of the ant brain consist of two pairs of structures called the pedunculate or mushroom bodies, each of which is a cupshaped mass of nerve fibers connected with the rest of the brain by a stem formed of similar fibers. The results of the investigations of several of the students of these bodies are briefly summarized in the following passage which I shall therefore cite: "It has been cus- 
tomary since the time of Dujardin to compare the pedunculate bodies with the cerebrum of vertebrates and to regard them as an organ of intelligence. Dujardin based his opinion on the fact that these bodies are largest and most elaborately developed in the social Hymenoptera. Leydig and Rabl-Rückard expressed a similar opinion. Forel (1874) first observed that these bodies are largest in worker ants, smaller in the queens and vestigial in the males, and as the worker was supposed to be the most, and the male the least, intelligent, this was regarded as additional evidence in favor of Dujardin's opinion. The condition described by Forel for the ants was affirmed by Brandt ( 1876 ) for the social Hymenoptera in general. More recently Kenyon (r896), after an elaborate study of the bee's brain, has reached a similar conclusion. He says : 'All that I am able at present to offer is the evidence from the minute structure and the relationships of the fibers of these bodies. This seems to be of no inconsiderable weight in support of the general idea started by Dujardin. For in connection with what was made known by Flögel and those before him and has since been confirmed and extended by other writers, one is able to see that the cells of the bodies in question are much more specialized in structure and isolated from the general mass of nerve fibers in those insects where it is generally admitted complexity of action or intelligence is greatest.' He also cites experiments of Binet (1894) which tend to show that in insects 'when connections between the dorso- and ventro-cerebron are destroyed, the phenomena afterwards observed are similar to those seen in a pigeon or mammal when its cerebral hemispheres are removed." "1

These facts seem to indicate that the ant has association areas and that they compare favorably with those of other insects. We may therefore expect to find that the ant is characterized by mental phenomena and manifests variations in behavior of an intelligent sort. I will speak of a few of these which are of significance for the social life of the ants.

The ants display the ability to recognize nest mates and aliens, and it seems quite evident that this is not an in-

W. M. Wheeler, op. cit., pp. 54-55. 
herited reflex, but is acquired. This is indicated by the facts that, on the one hand, ants can learn to accept ants of other species as nest mates, as is the case in mixed colonies and compound nests, and, on the other hand, that ants that have been separated as pupæ from their own colony will treat members of it as enemies. This could not be the case if ants inherited the tendency to treat only the members of their own species as friends and others as enemies. It is quite evident, therefore, that the recognition is due to an acquired habit of association, and there is a good deal of evidence that the recognizing is accomplished through the sense of odor. This is indicated by the experiment which has been performed of bathing an ant in the blood of crushed ants from another colony and then placing the ant back in its own nest, when it was attacked by the members of its own nest. Wasmann, who has studied this question at great length, expresses his opinion as follows: "The amicable reaction of ants to the odor of their own colony is not innate, but is acquired individually by the single ants. This individual acquisition occurs during the period in which the young, freshly developed worker begins to harden and take on her adult coloration. During this period her own definite individual odor first develops, and during this period there develops in her antennæ the olfactory sense, by means of which she is able to distinguish the odor of her own nest mates from those of other ants. Hence the ant's ability to distinguish between 'friend' and 'enemy' does not depend on inherited reflexes, but on the sensory perception of the olfactory impressions she receives during the first days of her life as an imaginal worker." 
Most observers believe that ants are able to communicate with each other. This seems to be indicated by the way in which they will gather at a place where one of them has found food or will retire from a place where some of their number have been killed or injured. To be sure, these phenomena may be explained in such a way that it is hardly accurate to speak of them as cases of communication. For example, an ant coming from food may leave the odor of it along her trail, by following which ants will find the food. Certain sounds and movements made by ants seem to convey meanings to other ants, or at least to influence their behavior. Some of these are vibrations of the antennæ, movements of the jaws, butting with the head, stridulation, etc. Furthermore, ants display their desire that their fellows act in a certain way by coercing them, as when they drag their queens about by the mandibles or carry one another to a nest or back to the old nest. There is great danger of interpreting these movements and sounds in too anthropomorphic a fashion. It is inconceivable that any of them have the conceptual significance that is possessed by words and sentences in human language. Probably most of them are instinctive in their character. But they influence the behavior of their fellows in a way which is almost, if not quite, intelligent in its character. Thus they are probably similar in their effects to the emotional cries in higher animals, which frequently influence behavior greatly.

I have spoken several times of the possibility that suggestion and imitation take place among ants. Many observers think that they have observed imitation among ants. This seems to be the case when a few individuals 


\section{Insect Societies: the Ants}

begin acting in a certain fashion and soon others begin acting in similar fashion, and this continues until the whole community is acting in the same way. It appears frequently as if the more initiative and alert members begin to act in a certain way and then are imitated by the others. This happens when a new condition faces the group and has to be met by some new form of behavior which may be devised by one or more of these more intelligent individuals. Thus we see the phenomena of invention and leadership displayed in a very rudimentary way.

Coöperation is frequently observed, and this may sometimes be the outcome of communication and of imitation. But it must be remembered, as has already been shown, that most of what appears to be coöperation among the ants is the result of a physiological division of labor and of common instinctive tendencies.

Still other aspects of the mental equipment of ants have been studied, as, for example, their memory and their capacity for learning by experience. All these characteristics are of some significance for their social behavior, but there is not the space to discuss them here. This chapter has shown that association among the ants is caused principally by anatomical polymorphism, which results in a physiological division of labor, and by certain instincts, especially the philoprogenitive instincts. The same is true of all the social insects. In the species which are not polymorphic association is caused in the main by certain instincts.

This description of the social characteristics of the ants must suffice as illustrating social phenomena among the invertebrates. 


\section{CHAPTER XIX}

\section{VERTEBRATE SOCIETIES}

Paleontological evidence of association among vertebrates, $364 .-$ Fishes, 365. - Amphibians, 370. - Reptiles, 371. - Difference between cold- and warm-blooded vertebrates, 372.-Birds, 373.The family, 374. - Mammals, 376. - Primates, 384. - Man, 387 .

LET us now make a brief survey of social phenomena as they appear among the vertebrates. In doing so, special attention will be given to the mammals, because man is a mammal.

There is some paleontological evidence that vertebrates have been social for a long time. This evidence is in the form of fossil remains distributed over small areas, which fact seems to indicate that when these remains were deposited, these species were living in bands and groups. These remains go far back in geological time, some of them dating from secondary time, while there are fossils dating from primary time indicating social life, but it is not certain that any of these are vertebrate. Such evidence with regard to the past is, however, necessarily very fragmentary. Most of the evidence must be secured from the study of living forms. But this evidence is of significance with respect to the past as well as for the present. If we find that social phenomena characterize species which are widely separated, and these phenomena are in part due to characteristics which are common to these species, we 


\section{Vertebrate Societies}

have some reason for believing that these social characteristics must have appeared in part at least before the point of divergence between these different lines of descent.

\section{FISHES}

The lowest class of the vertebrates consists of the fishes, so that we will consider first the social life of the fishes. Many species of fish live in shoals, frequently including a large number of individuals. In fact, some observers assert that this is true of most fishes. ${ }^{1}$ This may be due in part to certain external forces which have been mentioned in a preceding chapter; namely, temperature, pressure of the water, distribution of food, etc. But it is doubtful if these forces can explain it entirely in most, if in any, cases, for the area favorable for the life processes of the fishes is usually much larger than the area covered by one of these shoals, so that there must be still another force bringing them together. It is difficult to observe fishes in their natural habitat, so that explanations of their social behavior must necessarily be somewhat conjectural in their character. The fishes have only a slight mental development, owing to the slight development of the association areas of their brains. So that it is doubtful if there is much mental interaction between them.

But the sexual instinct is strong at certain seasons of the year, and the parental instinct appears to a slight extent in some species. When the sexual instinct manifests itself, it may bring the two sexes together. It, or rather certain curious instincts which sometimes accompany it, in certain species, causes them to do other things at the same

1 Cf. Brehm, Les merveilles de la nature, les poissons, Paris, p. 43. 


\section{The Science of Human Behavior}

time. For example, in the case of certain salt-water species just previous to the breeding time the males and females accompany each other up a river sometimes for a long distance to a place where the eggs are laid and hatched. The origin of these instincts is very obscure, but their utility to the species is very evident, since the young are much more likely to survive if born in the quiet waters of a river rather than in the sea or ocean. These instincts cause association between the sexes during this journey up the river. Most species of fish are oviparous, so that bodily contact is not necessary in order to impregnate the female. But bodily contact and rubbing between the sexes seems to take place sometimes and may stimulate the flow of milt from the male and of ova from the female. Since this takes place when the two are in close proximity to each other, the two fluids are almost certain to mingle, and some of the ova may be impregnated.

Out of the 8000 or more species of fish there are about I80 species which are viviparous. ${ }^{1}$ In these species the male has to come into bodily contact with the female in order to impregnate it, or at any rate it has to come near enough to inject its spermatozoa into the vent of the female. Then there are many species in which the male and female need never see each other. The female leaves its ova in the water. A male of the same species is attracted to them by their odor, and, instead of eating them, as it might the ova of another species, it sheds its milt over them, thus fertilizing some of them.

The above indicates very briefly the kinds of associa-

1 Alexander Sutherland, The Origin and Growth of the Moral Instinct, London, I898, p. 36 . 
tion between the sexes at breeding time. Let us now consider parental care among the fishes. There is very little of such care, especially on the part of the female. There appear to be only about ten species in which there is any maternal care, and in each of these cases the care is by means of an anatomical structure which serves to a certain extent as a sort of substitute for viviparity, so that maternal care is only to a very slight extent conscious. For example, in the six species of Aspredo the flat belly of the female becomes soft at breeding time. After the eggs have been fertilized by the male, she rolls upon them so that they stick to her, and she carries them in this fashion until they are hatched. In a few species the female has a pouch in which she carries her eggs until they are hatched. In the viviparous species the eggs are hatched either in the ovary or in the oviduct, there being no uterus or womb. In a few cases there seems to be a slight amount of maternal care after birth.

There is therefore very little maternal care among the fishes, and paternal care is more characteristic of them. The probable reason for this is that in most species the male fertilizes the eggs after they have left the female, and is therefore the last of the two parents to concern itself with them. As has been indicated, it is peculiarly sensitive to the smell and perhaps also to the sight of the eggs of its own species, and the attraction they have for it is sometimes strong enough to keep it with the eggs until they are hatched. During this time it cares for them in various ways. In some species it simply drives away marauders who would otherwise eat the eggs. In other species it takes the eggs into its mouth and carries them until 
they are hatched. The males of some species have pouches or soft bellies in which to carry the eggs, like the females which have already been described.

But the highest form of parental care displayed by the fishes is nest building, and the best example of this is found among the sticklebacks. The male stickleback builds his nest of weeds, straws, twigs, and leaves, sticking them together with mucus from his belly and weighting them with sand. Then he goes in search of a female, which he drives into the nest and keeps there until she has laid her eggs. $\mathrm{He}$ then watches over the eggs for from ten to thirty days until they are hatched, after which he protects them until they are able to wander off by themselves.

We see, then, that among the fishes are to be found variations from no parental care whatever to a certain amount of care. But in no case is there any association of any importance between parents and offspring, because, as has been indicated in the species which displays the most care, the young leave the father very soon, and the association before the separation takes place is of a very instinctive sort and therefore involving very little, if any, mental interaction. But this parental care is indirectly of great importance for social evolution. In the first place, it must be evident that where there is no parental care there must be an enormous waste of eggs, while as the degree of parental care increases the number of eggs needed to preserve the species will steadily decrease. This is well illustrated in the case of the fish. "Of species that exhibit no sort of parental care, the average of forty-nine gives $1,040,000$ eggs to a female each year; while among those which make nests or any apology for nests the number is only about 
10,000. Among those which have any protective tricks, such as carrying the eggs in pouches or attached to the body, or in the mouth, the average number is under rooo; while among those whose care takes the form of a uterine or quasiuterine gestation which brings the young into the world alive, an average of fifty-six eggs is quite sufficient." 1 In the second place, when there is parental care the period of helpless infancy can be lengthened, and the individual can develop to a higher stage before becoming mature. This is especially true with respect to the brain and nervous system, which will evolve in a more or less direct proportion to the degree of parental care. ${ }^{2}$ This more highly evolved nervous system will furnish the basis for more association and higher types of association.

It must now be evident that the life of fishes does not exhibit much association or high types of association between the sexes or between parents and offspring. There still remains the association in large shoals which has already been mentioned. This form of association is apparently not due to the sexual or parental instincts. It probably does not involve much mental interaction. It might be due to such an associative tendency or gregarious instinct as we have already discussed. But, as we have seen, the existence of such a tendency or instinct must be regarded as very doubtful. It may be that this form of association is due to a habit of associating together acquired by the young immediately after hatching, when, owing to the circumstances of their birth, they are thrown into each

1 Sutherland, op. cit., p. 40.

2 This is illustrated among the fishes by the fact that most of the viviparous species belong to the shark order, and the sharks have the largest brains in proportion to their weight of any fish. 
other's company. However, so far we can only conjecture as to the causes for this form of association displayed by fishes, and it is to be hoped that more study will be made of their behavior so that we may understand better the causes of the social phenomena displayed by these animals.

\section{AmpHibians}

The next class to be considered is that of the amphibians or batrachia, which includes such animals as frogs, toads, salamanders, newts, etc. Here we find much the same social phenomena as among the fishes. Perhaps the principal difference is that in most of these species the male clasps the female more or less tightly for a day or two at breeding time. The sexes are probably attracted together at this time by odor. The clasping is probably a stereotropic reaction due to irritation of the skin of the breast of the male at this time, which needs contact to allayit. Males have been known to clasp stones, sticks, etc., at this time, so desperate is their need to put themselves in contact with something. One great advantage arises from this reaction. When the female releases her ova, the male squirts his spermatic fluid over them so that the eggs stand a good chance of being fertilized. This increases the chances for survival, for example, over the fishes, two thirds of whose eggs, it is said, rot unfertilized. Some species are viviparous, in some of them the sperm fluid being sucked into the female through the genital opening. There is a little parental care, in which respect the batrachia are very similar to the fishes. In some species there are pouches or soft bellies in which the eggs are carried until hatched. 
These anatomical devices for protecting the eggs are sometimes on the males, sometimes on the females. There is very little in the way of nest building.

\section{REPTILES}

The next class is that of the reptiles, which includes the turtles, crocodiles, lizards, snakes, etc. With the possible exception of one genus (Sphenodon) containing two species, all reptiles fertilize their eggs internally, which increases greatly their chances for subsequent development. There is great variation among the different species as to how soon after fertilization the eggs are to be laid. Among the lower reptiles, such as the tortoises and turtles, the eggs are laid almost undeveloped. Among the higher reptiles, such as the snakes, the eggs are kept in the oviduct until half or three quarters hatched and sometimes until fully hatched. Thus the internal fertilization, the tough integument of the egg, and the universal reptilian instinct of concealing the eggs increase greatly the chances that the eggs will hatch and thus lessen greatly the number of eggs which are needed for the perpetuation of the species. There is very little parental care. It is said that the crocodile returns to the place where she has laid her eggs and aids the young to escape from them, but this is not certain. Some snakes incubate their eggs, but appear to give no care to the young after they are hatched, these being able to take care of themselves.

It must now be evident that the amphibians and reptiles are very little, if any, above the fishes in social development. Sexual relations are very temporary, and relations between parents and offspring are almost nonexistent, owing to the 
slight amount of parental care. In some amphibian and reptilian species, the individual members are sometimes to be found congregated together in considerable numbers, but whether this is due to social causes or not, it is hard to say. In any case there certainly is very little mental interaction, since the mental development of amphibians and of reptiles is very low.

\section{The Warm-Blooded Vertebrates}

We now come to the two highest classes of vertebrates; namely, the birds and the mammals. Both of these classes have undoubtedly sprung from a reptilian origin, but there is an important difference between them and the other vertebrate classes. The other classes are all of them cold-blooded ; that is to say, their body temperature is, within certain rather wide limits, approximately the same as that of the environing medium. But the birds and the mammals are warm-blooded; that is to say, their body temperature is kept by certain internal processes at a rather high point, which is usually above that of the environing medium. This characteristic of the birds and mammals is undoubtedly of considerable significance for their mental and social evolution. The warm-blooded type developed as a result of the development of the sympathetic nervous system, which regulates the vasomotor system in such a fashion as to keep the body at a uniform temperature by sending blood where more warmth is needed and stimulating the action of the sweat glands where the heat needs to be reduced. I have not the space to discuss the causes for this development here. As we have seen in an earlier chapter, the emotions arise out of the activity of the sympathetic 
system, so that the development of that system means the development of the emotional nature of these classes of animals. So that the emotions involved in sexual, parental, and wider social relationships now begin to play a much greater part.

\section{BIRDS}

Among birds the eggs are fertilized internally and are partially matured inside the female, but are laid before they are hatched, so that all birds are oviparous. There is a great deal of variation among the birds as to the degree of parental care, as in all these other classes. Generally speaking, the degree of parental care varies with the degree of intelligence. The least intelligent are the running birds, such as the ostrich, the emu, the rhea, and the cassowary. These species make no nest beyond digging a hole in the sand or soft earth in which the eggs are laid. The incubating may be done by both parents, but in many of these species is done in large part or entirely by the male, so that paternal care preponderates over maternal care among these species. This is reminiscent of the lower classes, in which, as we have seen, paternal care is frequently predominant. There is very little care of the young, since they are almost, when not entirely, self-dependent from the time of hatching.

The next group of birds, which are of medium intelligence, includes the web-footed, the stilt-legged, the pheasant-like birds, and the pigeons. Some of these species make no nests whatever. The others make nests most of which are rather rude in character. In all these species the female does most of the brooding, but is usually assisted by the male. The young are hatched more or less dependent and 
have to be cared for during varying periods of time before they can shift for themselves.

The most intelligent birds include the birds of prey, the owls, the woodpeckers, the parrots, the sparrow-like and the finch-like birds. There are 1670 genera of these birds, and they all display a great deal of parental care. Most of them make nests. Those of them which do not make nests lay their eggs in safe places which are sometimes better than nests. They display much intelligence in adjusting their nests to local conditions instead of invariably building them in the same way. The brooding is usually done by the female, but the male always remains with her, feeding and protecting her. The young are always hatched helpless and have to be warmed and cared for in every other way for some time by both parents. Even after they are able to care for themselves, the young of certain species remain with their parents, sometimes until they are ready to mate and found homes for themselves.

Among the birds we see the family making its appearance. Among the running birds it is very weak. These birds are frequently to be found in small groups of males and females in which a certain amount of temporary pairing may take place. Sometimes a single male will be accompanied by several females. But no unions of any duration between individuals of the two sexes seem to be formed, and, as we have seen, there is little parental care. Among the birds of medium intelligence the family makes its appearance in very definite form. Here we find parental care through the period of helplessness by at least one parent and frequently by both parents. When the latter is the case, we have a union between the two parents which lasts 
at least as long as the breeding season. Among the birds of high intelligence there is always parental care by both parents, and very frequently pairing takes place for life. Thus we find the monogamous family fully developed among the birds, with permanent conjugal relations and relations of considerable duration between parents and offspring.

It is hardly necessary to emphasize the significance of these conjugal and these parental and filial relationships for social evolution. In the course of a long-continued or permanent conjugal relationship there can develop a degree of coöperation which would not be possible during a brief and temporary connection. During such a long connection a great deal of mental interaction must necessarily take place. We cannot know to what extent the birds are able to communicate with each other, but some of their notes seem to have specific meanings, while in the form of suggestion and imitation there is much mental interaction. Then the long-continued relations between the parents and offspring must have great influence over the young. During this time the young can learn many things from the older birds, and thus a certain amount of social tradition in the form of ways of doing things, as to what things are to be avoided, etc., can be transmitted from generation to generation.

But perhaps the principal social significance of this family life is that it is a preparation for a wider type of association. Many species of birds live in flocks and other large groups. In family life is acquired to a certain extent the habit of association which fits the young for life in the flock. It must be noted, however, that the family 
may be, and very frequently is, antagonistic to the wider forms of association. The attraction between the sexes and the jealousies which arise therefrom frequently result in the breaking up of the larger group, so that it is frequently hard to determine whether the family is more of a force for or against the wider forms of association. But this is a subject which will be discussed more fully later with respect to mammals as well as birds.

Various forms of social relations arise in these larger groups. Coöperation for warning against danger and for defense against danger takes place to a great extent. Migrations usually take place in these large flocks. There is a great deal of play in the form of singing, caressing, etc., which goes on in these flocks. In the case of most, if not all, of the higher birds that do not come together in large groups there is some special reason for it. Certain species have to maintain solitary habits in order to escape the observation of their enemies, just as other species have to combine in order to defend themselves against their enemies. Certain birds of prey have to live solitary lives in order to secure their food, since it would be impossible for a large number of them to secure sufficient food at one place.

\section{MaMmals}

Let us now survey briefly the social characteristics of the mammals. As has already been stated, the mammals, like the birds, have a reptilian origin, but it is impossible to determine at what point the mammalian line of evolution diverged from the reptilian. This point, however, was undoubtedly different from the point at which the avian line of evolution diverged from the reptilian. The lowest 
mammals give indications of their reptilian origin, for they are to a certain extent transitional forms. The lowest mammalian order is that of the monotremes. This order, which includes the duckbill or platypus (ornithorhynchus) and the spiny anteater (echidna), is reminiscent of the reptiles in several ways. It is not completely viviparous, inasmuch as the young are sometimes born while still inside the covering of the egg. The young are, however, born or hatched weak and helpless and only partially developed and not active and independent like most of the young reptiles. Parental care is therefore absolutely necessary for the survival of the young, and this care is always maternal. The female has two pouchlike folds into which she lifts her young. Inside these pouches are glands from which oozes a milky secretion upon which the young feed. These are, in all probability, modified fat glands which form rudimentary milk glands, though Gegenbauer thought they were modified sweat glands. The mother carries her young until they are able to shift for themselves. Conjugal relations among the monotremes are apparently of the briefest sort, occurring only for the purpose of sexual intercourse. There seem to be among them no social relations of a wider sort, since they form no larger groups and seem to be indifferent to each other when brought near together.

The next higher group of mammals is that of the marsupials. This group includes two orders, the first being represented by the opossum and the second by the kangaroo. The mammals in this group are wholly viviparous, but the young are born partially undeveloped and are placed immediately by the mother in a pouch or marsu- 
pium, as it is called, from which the group derives its name. Inside this pouch are the mammary glands, from which hang long, slender teats, through which the young feeds until it is able to leave the pouch. Some of the lower species of marsupials lack this pouch, or have it only in a rudimentary form. But all of them are viviparous and have mammary glands. With regard to conjugal association and wider forms of association, the marsupials seem to differ a good deal amongst themselves. In most of the species conjugal relations seem to be solely for the purpose of sexual intercourse, but there are certain species in which pairing for a longer period of time takes place. Some of the species are somewhat gregarious, while others are quite solitary. But in the gregarious species the social relations seem to be of a low order and do not involve much mental interaction.

We now come to the placentalia, which include most of the mammalian orders. These orders are named from the placenta in the womb through which the young are nourished during the uterine life. The young are always born fully developed, but in many orders are very helpless and need a great deal of parental care for a considerable period of time. The placentalia are divided into the nondeciduate and the deciduate orders, and this division has some significance with respect to the degree of parental care needed. In the nondeciduate orders, which include the ungulata, the cetacea, and the sirenia, the period of gestation is long and the young are born active and usually able to move about, but needing the mother's milk and parental protection for some time. In the deciduate orders, which include the edentata, the rodentia, the insectivora, the chiroptera, the carnivora, and the primates, 
the period of gestation is shorter on the average, but the young are born much more helpless, so that they are in need of much more parental care. Perhaps owing to this greater need for parental care after birth, some of the deciduate orders have progressed much farther in social evolution than have the nondeciduate orders, though it is also true that some of the deciduate orders stand very low among the mammals both mentally and socially, as, for example, the edentata and the insectivora.

On account of lack of space, I can touch only very briefly upon the social characteristics of these mammalian orders. In all of them a certain amount of parental care is necessary in order to suckle the young and also frequently in order to protect and warm them. There is great variation among them as to the extent to which they display conjugal relations and social relations of a wider sort. The edentata and the insectivora, which are of the lowest grade mentally, seem to have practically no conjugal relations or social relations of a wider sort. The marine mammals, namely, the cetacea and the sirenia, represented by the whales and the porpoises, are gregarious and seem to be bound together by strong social bonds. within their herds, but it is doubtful if they have strong conjugal relations. The bats (chiroptera) are gregarious, but appear to have no conjugal relations other than that of sexual intercourse. The sexes are usually segregated from each other in large flocks. The rodents vary greatly amongst themselves as to their social characteristics. Conjugal relations seem to be very weak among them, the pairing usually lasting only during the period of sexual excitement, and the male rarely ever taking part in the care of the 
young. Some of the genera are gregarious and coöperate in digging and building, as, for example, the beavers and some of the rabbits, but other genera are quite solitary in their manner of life.

The order of the ungulata is the highest order of the nondeciduate placentalia. The members of this order are usually herbivorous and hoofed. It includes a large number of genera, in which are the horse, the ox, the sheep, the deer, the elephant, and the rhinoceros. The ungulata are almost without exception very gregarious and in their natural state live almost always in large herds. This is quite in keeping with their manner of securing food, inasmuch as they are herbivorous and hence are not beasts of prey. There is very little in the way of conjugal relations, as the males and females live together the year around without segregating into pairs. Within the herd, however, the males usually take upon themselves the task of protecting the females and young. The degree of gregariousness displayed by the ungulata is very great. Galton has given a classic statement of it in his description of the South African ox. He says that "the ox cannot endure even a momentary separation from his herd. If he be separated from it by stratagem or force, he exhibits every sign of mental agony; he strives with all his might and main to get back again, and when he succeeds, he plunges into its middle to bathe his whole body with the comfort of closest companionship." 1 This gregariousness is very useful for purposes of protection, since if the ungulate attempted to live a solitary life it would probably be destroyed by a beast of prey. But as Galton points out,

1 Francis Galton, Inquiries into Buman Faculty, New York, 1883, p. 71. 
this extreme gregariousness destroys the self-reliance of the members of the herd and thus checks their mental development. With the exception of the few that act as leaders of herds, these animals act in accordance with the movements of the herd. Their craving for companionship seems to be due largely to habit and the discomfort they experience when separated from familiar things. The basis for their gregariousness is negative principally, and not positive, as it is to so large an extent with the primates, who seek each others' company for what they can do to and with each other.

The next order to be considered is that of the carnivora. It has been stated that this order belongs to the deciduate placentalia. Therefore its young are born quite helpless and have to be cared for during a considerable period of time. It is therefore to be expected that strong parental feeling will be displayed by this order. And this is to be found, at least so far as the mother is concerned. In all the genera of this order the female devotes herself to the care of her young throughout the period of their helplessness and displays a strong affection for them. But conjugal relations in this order are very weak and in many genera do not exist at all aside from sexual intercourse, so that there is very little care of the young by the male. Furthermore, there is not very much association of a wider sort, since not many of the carnivorous species form packs or herds. The gregarious species are to be found principally in the families of the dog, the seal, and the walrus, while the families of the cat, the civet, and the raccoon, and the subfamilies of the badger and the weasel are more or less solitary in their mode of life. This lack of gre- 
gariousness is probably due to the facts that, as they are well armed by nature, they do not have to unite for protection, and that as beasts of prey they have to scatter in order to find their food.

But despite the solitariness of the manner of life of most of the carnivorous species, they display a good capacity for leading a social life when the opportunity of leading one is offered them. This is indicated by the fact that many household pets domesticated by man have been carnivorous animals. I need not stop to describe the social characteristics of the dog and the cat, while many other carnivorous animals have been domesticated almost, if not quite, as successfully, as, for example, the raccoon, the badger, the weasel, the seal, etc. This seems like a curious fact and is one which it is perhaps a little hard to explain. At least two reasons for this phenomenon can, however, be indicated, and these may be sufficient to explain it satisfactorily. In the first place, it must be noted that the carnivora usually give birth to several offspring at the same time, so that there are several young ones being brought up at the same time in the lair. As soon as they are old enough to do so, they begin to play together, and in these playful relations with their fellow offspring as well as in their relations with their parents they acquire social habits which they never entirely lose. But in course of time, when they are old enough, they have to go out in search of food, which results in breaking up this association. Or jealousy aroused as a result of the awakening of sexual feeling may destroy this association.

In the second place, and this is probably the principal reason for the facility with which they adopt social hab- 
its, the carnivora undoubtedly form the most intelligent mammalian order with the exception of the order of primates. This is probably due in large part to their predatory mode of securing food, which places a high premium upon skill and cunning. The carnivora are undoubtedly subjected in their natural mode of life to a powerful selective process which tends to preserve the more intelligent and to eliminate the less intelligent. In this respect they are in strong contrast to the ungulata, whose preservation depends largely, as we have seen, on their conformity to the behavior of the herd. It is therefore not surprising that the ungulata are relatively unintelligent and stupid as compared with the carnivora. Its relatively high intelligence therefore enables the carnivore to adjust itself to a social life when it is given the opportunity. It is amenable to suggestion and will imitate and enter sympathetically into the feelings of those surrounding it. It is of course necessary in order that it may do these things that it be relieved for the time being from the necessity of securing food, for otherwise its predatory instincts will control its behavior. Such carnivora as the dog and the cat, which have been domesticated for many centuries, have been put through a long selective process which has weeded out the unsocial and has preserved the social. This accounts in part for the social characteristics of these animals. But there must have been a basis upon which to build in the case of these animals, for a totally unintelligent animal could not possibly be domesticated, inasmuch as it would be incapable of learning. Furthermore, carnivores of other species are constantly being domesticated from the wild state so successfully as to establish very close relations 


\section{The Science of Human Behavior}

between them and their human masters. This suggests the part which may be played by intelligence in making animals social. This appears more fully in the highest order of mammals, the primates, and will be discussed more fully in the next chapter.

\section{Primates}

We now come to the order of the primates, which is of peculiar interest to us, inasmuch as it includes man. The period of gestation is on the average much longer in this order than it is in any other order. The young are born more helpless than in any other order. Consequently the maternal instinct is highly developed in this order. Owing to the long period of gestation and of infancy, the nervous system develops greatly, thus making possible the intellectual development which furnishes a basis for the high degree of social evolution attained by this order.

The order of primates is usually divided into two suborders; namely, the lemuroidea and the anthropoidea. The lower suborder, the lemuroidea, contains about a dozen genera, including the true lemurs (family lemuridæ), the aye-aye, and the tarsius spectrum. The young of the lemurs are not as helpless at birth as those of the other primates. For example, immediately after birth they are able to climb up the mother's body to the teats, which are located on the breast. This pectoral location of the mammary glands, which is rare in other orders, is of some significance in the primates, for it enables the mother to hold her young in her arms while it is suckling, thus increasing the degree of care and protection she can give it. The young must, however, be fed and protected for some 
time before it can shift for itself, consequently the maternal feeling is very strong. It is hard to determine to what extent conjugal relations exist among the lemuroidea. It is said that the two lowest genera, the aye-ayes and the tarsius spectra, are generally found in pairs. But most of the lemuroidea are very gregarious and live in groups of considerable size in their arboreal haunts. So that it is doubtful if conjugal relations are very strong among them. They are not very intelligent as compared with the rest of the primates, so that there is probably not very much mental interaction between them.

The suborder of the anthropoidea includes five families: the hapalida, which includes the marmosets; the cebida, which includes the howling monkey, the squirrel monkey, the spider monkey, the capuchin monkey, etc.; the cercopithecida, which includes among others the baboons and the macaques; the simiida, the members of which are frequently called the anthropoid apes, which includes the gibbons, the orang-utans, the chimpanzees, and the gorillas; and the hominide, which includes only man.

The first three families are monkeys with tails, the first two being platyrrhine and the third being catarrhine. Together they form the lower group of the anthropoidea. All of these monkeys of the three lower families are very gregarious, and most of them live in groups of different sizes. Perhaps for this reason conjugal relations are not very strong, but the males seem to have a strong affection for the young and protect and care for the young of their groups. Polygyny exists among some of these monkeys, and the connection between the male and his wives is usually permanent.

2c. 
The simiidæ or anthropoid apes are more like man than any other living beings. The infant ape is the most helpless of all new-born animals, with the exception of the human babe. Consequently, maternal affection is strong in all the genera of this family, and the mother cares for her young for a long period of time. The lowest genus is that of the gibbons (hylobates). These apes live in bands, sometimes numbering as many as one hundred. It is not certain whether the sexes live in pairs within the band or not. But in any case the males share in caring for the young. It is said that when on the march the young are borne by adults of their own sex. That is to say, a young male is carried by a male adult, while a young female is carried by its mother. It is hard to explain this curious custom. The next genus (simia) contains only one species, the orang-utan. It is not very gregarious, since it is apparently never seen in large groups. The male seems to lead a solitary life usually, but it is said by some observers that he keeps near the mother and her young and watches over their safety. The chimpanzee (anthropopithecus or troglodytes) lives in small bands and seems to have a highly developed capacity for social feeling, as is revealed when it is domesticated. The males watch over their mates and their young with great care. The gorillas (gorilla) are never found in groups, except when a few young gorillas are joined together apparently before they have mated. A male and female with their young are usually found together. Sometimes a male has more than one female. The relations between mates and parents and offspring seem to be very close. Despite its reputation for fierceness, the gorilla seems to have a strong capacity for affec- 
tion, which has been revealed on the few occasions on which it has been tamed.

\section{MAN}

The last family of the anthropoidea is that of the hominidæ, which brings us to man (homo). I shall discuss elsewhere the characteristics of the branches of mankind which have progressed somewhat on the road of human social evolution. The social characteristics of primitive man it is unfortunately rather hard to determine with certainty, inasmuch as there are very few examples of primitive man remaining. The period of gestation is longer in proportion to his size for man than it is for any other living being. The human infant is perhaps the most helpless of any species and needs parental care for a long period of time. Human parental instinct is therefore very strong. Primitive men appear usually to have lived in small groups ranging in size from twenty to forty or fifty and perhaps sometimes larger. Within these groups there seems always to have been a relative degree of promiscuity. But upon the basis of this promiscuity there has sometimes arisen monogamy and sometimes polygyny, very rarely if ever polyandry among the most primitive men. Thus it appears that primitive man was more gregarious than the anthropoid apes, with the possible exception of the gibbon, and that conjugal relations were not usually as strong among primitive men as they are among some of the anthropoid apes, as, for example, the gorilla, and as they are among some of the higher birds. This would seem to relate man with respect to his social characteristics more closely to the monkeys than it does to the anthropoid apes. This may be corre- 
lated with the fact that in his physical characteristics man may in some ways be more closely related to the monkeys than he is to the anthropoid apes. If this is true, it would be due to the fact that man diverged from the main stem of the primates at a point nearer the monkeys than the apes. However, this is very uncertain, but it seems to be the opinion expressed by Petrucci in the following passage: "Hence it is that the examination of the animal types which have differentiated from the line of evolution which leads from the primatoid prototype to man shows that the lemurs, using these as a type of comparison, display life in bands and grouping by families, that this much-accentuated social characteristic is continued in the monkeys, which diverge from this line, that it is still continued in man, while in the anthropoid apes it tends to disappear and is clearly displayed only by the gibbons, who resemble in this respect the monkeys. Therefore man and the gibbon occupy each a place by himself in the heart of the order of primates, and man shows himself to be less divergent from the primitive primate and primatoid type in his social characteristics than the other anthropoids (except the gibbon). This is in accordance with the place that his structure gives him and from this very general predominance, even in divergent types, of the social characteristic from which only certain anthropoid apes escape we can conclude for the present that these tendencies are inherited in man from the primatoid prototype for which the lemurs furnish us a standard of comparison." 1

Even if it be true that in some of his physical charac-

1 Origine polyphyletique, homotypie et non comparabilité directe des sociêtes animales, Brussels, Igo6. 


\section{Vertebrate Societies}

teristics man resembles the monkeys more than he does the apes, this does not necessarily mean that he is inferior to the apes. As a matter of fact, his brain development is much superior to that of the apes, and in an earlier chapter have been discussed some of the reasons for this superiority, though we cannot know all these reasons. On account of this superior brain development, as well as for other reasons, the line which diverged from the common primate stock and evolved into man has progressed much further mentally and socially than the line which diverged from the common stock and evolved into the anthropoid apes. 


\section{CHAPTER XX}

\section{THE FACTORS OF SOCIAL EVOLUTION}

The causes of association, 390. - The polyphyletism of animal societies, 39r. - Utility for survival as a controlling factor in social evolution, 392. - The reasons for man's superior social evolution, 393. - Environmental forces for association, 395. - Instinctive forces for association, 395.- Is there a gregarious instinct? 395.The sexual instinct, 396. - The reproductive instincts, 397. - The parental instincts, 397. - The utility of parental care, 397. - Conjugal relations, 399. - The family, 399. - Wider forms of association, 40I. - The antagonism between the family and the horde, 403. The family as preparing the way for wider forms of association, 404 . - Emotional forces for association, 406. - Intelligent forces for association, 407. - Imitation, 407. - Recognition, 407.- Communication, 409. - Language, 409. - The formation of categories, 4II. Meeting places, 4I4. - Leadership, 415. - Theories of social evolution, 416. - Theory of the instinctive origin of society: Petrucci, McDougall, 4r 7. - Theory of the emotional origin of society: Adam Smith, Sutherland, $4 \mathrm{I} 8$. - Theory of the intellectual origin of society: Giddings, Kropotkin, Tarde, Durkheim, 420. - The complexity of the factors in social evolution, 421.

IN the last two chapters a brief survey has been made of the social characteristics of representatives of the principal branches of the animal world. I shall now make an attempt to describe the principal forces which caused the origin and early stages in social evolution.

The preceding discussion has shown how manifold have been the causes of association. Certainly it is not to be attributed solely to one gregarious instinct, if indeed 
such an instinct exists at all. We have seen that there are at least four different kinds of causes of association; namely, environmental, instinctive, emotional, and intellectual causes. The information which has been furnished with respect to these causes will be summarized briefly in this chapter.

\section{Polyphyletism of Antmal Societies}

The preceding discussion has also shown the variations in the extent to which life is social, even among species which are closely related to each other. For example, we have seen that within the family of the simiidæ the gibbons are very gregarious, while the gorillas lead a relatively solitary life. This discussion has illustrated the polyphyletism of animal societies referred to in an earlier chapter. It has shown that social evolution, like other forms of evolution, is not entirely linear, but is to a certain extent multiple and divergent. It has shown that social evolution is not entirely correlated with organic and intellectual evolution. This was illustrated by the fact that the order of ungulata is more gregarious than the order of carnivora, notwithstanding the fact that the carnivora are much more intelligent than the ungulata. It was illustrated by the fact that many avian species are more social than many mammalian species, even though they have not evolved as far organically. This discussion has raised the question as to the degree of homology and parallelism which exists between the phylogeny of animal societies and the phylogeny of the species of the animals which constitute these societies. That there must always be a certain degree of such homology and parallelism is quite evident, but that 
the two phylogenetic series may vary to a considerable extent is also quite evident.

\section{The Controlling Factor in Social Evolution}

The explanation of these variations is, I believe, to be found in the fact that the controlling factor in determining whether association is to exist and the extent to which it is to exist is its utility for survival. A species is likely to become social if social characteristics will aid it in its struggle for existence. That is to say, the individuals possessing social characteristics will be selected for survival, and thus the species will become more and more social. As we have seen, there are various ways in which association will aid in the struggle for existence. It may aid in securing food, or in caring for the young, or as a means of defense against enemies. On the other hand, as we have seen, social characteristics have little or no utility for many species and frequently hamper them in the struggle for existence, as, for example, predatory animals who need to be alone to secure their food, and certain animals whose safety from their enemies depends upon their being as inconspicuous as possible.

Lloyd Morgan has described very graphically how sociability depends upon utility in the struggle for existence in the following words: "The assertion that the fittest are the most sociable animals, that sociability appears as the chief factor in evolution, and that unsociable species decay, is not likely to be accepted without qualification by zoölogists. What grounds have we for saying that the solitary wasps are less fit than the social wasp? Each has a fitness according to its kind. Can it be maintained that the unsocial tiger is less fit than the social jackal? And 
can it be said that tigers, which are reported absolutely to swarm in Java and Sumatra, exemplify the decay of an unsociable species? Is it seriously contended that the hawk, which may be successfully mobbed by a number of wagtails, is less fit than his more social assailants? And are the unsocial raptorial birds decaying species? Such questions might be asked by the score. And the answer in every case is that the social and unsocial alike are fitted to their several states of life." 1

\section{Reasons for Man's Superior Social Evolution}

It goes without saying that utility for survival has been the controlling factor in the social evolution of man just as much as in the case of any other social species. Human social evolution is no more teleological than any other kind of evolution. Man is very social, because he is weak as compared with many species which prey upon him, because his young are born very helpless and remain so for a long time, and because he has a highly developed nervous system which furnishes the basis for a high degree of intelligence, thus making possible a high degree of mental interaction, from which result a good deal of pleasure and a great deal of coöperation and mutual aid in the form of a social division of labor which facilitates greatly the securing of food and the other necessaries of life. If it had not been for these advantages accruing from association, man would not have been social, despite the belief of many that it was preordained that man should reach the highest point in mental and social evolution and should thereby dominate the rest of the animal world. Darwin, in his classic discussion of this subject, has ex-

1 Animal Behaviour, London, 1908, p. 229. 


\section{The Science of Human Behavior}

pressed himself as follows: "In regard to bodily size or strength, we do not know whether man is descended from some small species, like the chimpanzee, or from one as powerful as the gorilla; and, therefore, we cannot say whether man has become larger and stronger, or smaller and weaker, than his ancestors. We should, however, bear in mind that an animal possessing great size, strength, and ferocity, and which, like the gorilla, could defend itself from all enemies, would not perhaps have become social; and this would most effectually have checked the acquirement of the higher mental qualities, such as sympathy and the love of his fellows. Hence it might have been an immense advantage to man to have sprung from some comparatively weak creature." 1

In order, therefore, to explain fully the social or unsocial characteristics of a species, it would be necessary to know the whole history of its phylogenetic evolution and all the selective forces of its environment to which it has been subjected. In other words, it would be necessary to have the same information that it is necessary to have to explain its anatomical, physiological, and psychological characteristics. By saying this, however, I do not mean to imply that social evolution is entirely correlated with organic and mental evolution, for, as we have seen, owing to forces of the environment and other factors, there may be variations in the social characteristics of a species which are not accompanied by correspondingly great organic and mental changes. But inasmuch as there is a general correlation, the data furnished by biological and psychological research are of the greatest value for making possible a more detailed explanation of social evolution.

1 The Descent of Man, 2d edit., London, I89o, pp. 63-64. 


\section{EnVtronmental Forces}

Let us now review briefly the forces for association which have been discussed. The first group is that of the external, environmental forces, such as temperature, the distribution of food, etc. Enemies are sometimes included among these forces, though these are perhaps social forces in an inverted sort of a way, inasmuch as relations with enemies may and usually do involve a certain amount of mental interaction. These external forces which bring about association are sometimes called socializing forces. Criticism has been made of this term by some who have contended that these forces also are social forces, inasmuch as they would not bring about association were it not for certain characteristics of the animals upon which they act. It is true that a certain temperature will not bring about association unless certain animals are adapted to that temperature and that a certain kind of food distributed over a certain locality will not be a force for association in that locality unless certain animals are so constituted that they can subsist on that food. But notwithstanding these considerations, there is still some reason for distinguishing between these external forces and the forces for association which arise out of the mental interaction of living beings.

\section{InSTINCTIVE FoRCES}

The next group of forces for association to be considered is that of the instinctive forces. I have stated the theory of some writers that there is a specific gregarious instinct and have indicated the arguments against this theory. It is indeed hard to see how such an instinct can exist, because 


\section{The Science of Human Behavior}

it is hard to see how it could have come into existence. The exposition of the nature of instinct in a previous chapter has shown that no instinct is likely to come into existence unless it performs some definite service which is of utility in the struggle for existence. A fully developed gregarious instinct would be of utility to many species, but I do not know what forces would nurse it through the early stages of its development before it had selective value. It is evident that such an instinct would necessarily have to be very complex in its character. It would have to be a reaction of the whole organism to the whole organism of another member of the same species. To be aroused, it would probably have to be stimulated through several senses. So that the first stages of its development would not be so likely to have utility and selective value as the first stages of simpler instincts. It is barely possible that a gregarious instinct might appear as the result of the combination of several simpler instincts, but it is doubtful if this has ever taken place.

But while we may not believe in a specific gregarious or herd instinct, still there are a number of instincts which may be called gregarious because they cause association. The first of these is the sexual instinct. This instinct is aroused by very definite stimulations in the form of tactile excitations, odors, colors, sounds, movements, etc. It has the highest selective value because it is essential to reproduction and therefore to the preservation of the species. It does not, however, always involve association. As we have seen, in some of the fish species the two sexes do not need to come into association with each other, for the female will release her eggs independently of the males, and the sexual instinct 
of the male will be stimulated by the odor of the eggs so that he will release his sperm cells. But in many species contact or, to say the least, close proximity is necessary for reproduction, and in these cases the sexual instinct results in association.

Some writers speak as if there is a specific reproductive instinct, but it is very questionable as to whether there is any such instinct. The reproductive process is started by the sexual instinct and is continued and its success assured in many species by the parental instinct. So that we may speak of the sexual and parental instincts and of any other instincts which aid in the process of reproduction as the reproductive instincts.

The next instinct to be considered is the parental instinct. This instinct, however, manifests itself in many different forms in different species and frequently involves several more or less distinct instincts, so that it would perhaps be more accurate to speak of the parental instincts. All of these instincts involve care of the young, and we have had numerous illustrations of them earlier in this chapter, as, for example, nest building, incubating, suckling, etc. Such care involves association between the parents and the young, so that these instincts are a force for association. But indirectly, also, they are a force for association. As we have seen, as parental care increases, the number of eggs needed for the perpetuation of the species decreases. This is indicated well in the following passage: "A steady diminution in the number of offspring as parental care increases is a prime feature of development. In fish, as already stated, the average of seventy-five well-distributed and typical species is 646,000 eggs, but in the class amphibia 
the average of the twenty species for which information is to be had, is no more than 44I eggs, while in the class of reptiles, the average of thirty-nine species is only seventeen. The birds represent a much higher standard, and they, as the average of more than 2000 typical species, give only a trifle over five eggs per annum for each female. A still higher rank is reached in the mammals ; as the average of eightytwo typical species they have only 3.2 offspring to each female every year, and within the mammals as a class the same progressive diminution is to be seen; all the higher orders taken together average only I.3 young ones each year, while the apes and mankind do not exceed one every two years." 1

This reduction in the number of eggs needed for the perpetuation of the species results in a great saving of energy and of nourishment for these species, and this saving can be utilized for the higher development of the species. $\mathrm{Pa}$ rental care also makes possible a lengthening of the period of infancy, thus making it possible for the individual to reach a higher point before maturity. In many species this longer period of development results in a more complex nervous system, which furnishes a basis for a higher degree of intelligence, which in turn makes possible higher forms of association. Thus the parental instincts resulting in parental care prepare the way for higher forms of association which otherwise could not possibly have come into existence.

1 A. Sutherland. The Origin and Growth of the Moral Instinct, London, I898, Vol. I, p. 4 I. 


\section{The Family}

Let us now turn to the conjugal relations between the parents. We have seen that, in order to reproduce, the sexes do not have to come together in all species. In many species, probably the great majority, the sexual instinct manifests itself only at certain times, usually at certain seasons of the year. In these species, therefore, the sexual instinct acts as a force for association only at this time, which is called the rut. Consequently, if the sexes associate the rest of the time, it will be due to other forces. We have seen that parental care may be exercised by the one or the other parent, or it may be exercised by both parents. If it is exercised by only one parent, it is of no significance for conjugal relations. But if it is exercised by both parents, it may lead to conjugal relations between the same pair through a long period of time and sometimes permanently. This will be the case when both parents have the same parental instincts, which will lead them to do the same things at the same time and therefore together. For example, this is well illustrated in the case of the nest-building instinct. At the time of the rut along with the sexual instinct is aroused this instinct in both sexes, so that the mating of a pair leads both of them to set about the constructing of a nest. When the nest is completed and the eggs have been laid, there appears the incubating instinct in both parents, though it is usually strongest in the female, while the male displays more specialized instincts which lead it to act as a protector and a purveyor of food. These instincts are probably of the nature of the chain instincts which have been described in the chapters on instinct. , 
That is to say, the expression of one of these instincts acts as a stimulus for the next one, so that a series of instincts may manifest themselves as if they formed one instinct. So it is that the presence of these parental instincts in both parents may lead to conjugal relations lasting through the breeding season. Why it is that in some species these instincts are transmitted to both parents and in other species to but one parent is a problem of heredity which cannot be discussed here.

The sharing of the same parental instincts by both parents may therefore be the explanation of conjugal relations lasting through the breeding season. But this does not explain the permanent conjugal relations which exist among some of the birds and a few of the mammals. As to whether or not it is due to an instinct, it is impossible to say. But it seems much more likely that it is due to a habit of associating together which is acquired during the breeding season when they are held together by these instincts. It is conceivable that among the higher birds and mammals there may be a sufficient degree of mental interaction to cause personal relations strong enough to hold individuals together, even when there is no other force for association at work.

Out of these relations between parents and offspring and these conjugal relations there develops the social group called the family. There has been some difference of opinion as to what constitutes a family, but the majority of writers now seem to think that a family is constituted whenever a relation of some duration exists between one parent and its offspring. The family may therefore consist of the mother and the offspring, or of the father and the 
offspring, or of both parents and the offspring. In the few species in which polygyny exists, the family would consist of the father and the mothers with their offspring. I know of no species other than the human species in which there is any polyandry.

It is evident therefore from the facts stated in the last chapter that the family can hardly be said to appear below the birds, since there is no relation of any duration to speak of between parents and offspring among the reptiles, amphibia, and fishes, or among the invertebrates. But the family reaches a high degree of development among the birds and mammals. As we have seen, in a good many species of birds mating is for life, and the same is true of certain mammals. But it is doubtful if there is any species other than the human species in which permanent relations are maintained between parents and offspring. In all other species the young leave their parents when they are full grown and are ready to mate, and the recollection of their relationship in all probability soon fades from their memory. Among men, on the contrary, this relationship is usually remembered permanently and has an important influence upon social organization.

\section{Wider Forms of Association}

Let us now turn to wider forms of association than the family. As we have seen, there are a good many species that live in groups larger than a family. These groups vary greatly in size between the different species. For example, we have seen that an ant colony may number tens if not hundreds of thousands. Fishes and birds live in shoals and flocks which may number many thousands. 
Among the mammals the ungulata live in herds which may include thousands, and some of the carnivora form packs which number hundreds if not thousands. It goes without saying that the family must be made up of members of the same species. The same is usually true of these larger groups, though there are some exceptions in the form of the commensal and parasitic associations which have been discussed.

The causes for these wider forms of association have already been briefly discussed in this chapter. I wish at this point to discuss a little more fully the relations between these wider forms of association and the family. It may appear most probable that these larger groups are made up of families. This is true to a certain extent in some cases. For example, a herd of ungulata may be made up in part of females who are accompanied by their young whom they are suckling until they are able to feed on grass. But aside from this temporary relationship between the mothers and their young, there is not likely to be anything like a family in a herd of ungulata. And the same principle holds wherever large groups of this sort exist permanently. It may sometimes happen that birds of a species which mate permanently may join together temporarily for their migrations, but it is evident that as soon as they reach their breeding ground they must break up into couples in order to found their nests. Again, after the young are hatched and are somewhat grown there may arise between the young of several families a form of association not based upon sexual attraction which might develop into one of these permanent associations of the wider sort, were it not for the fact that when the sexual instincts manifest themselves, 
sexual jealousy will appear and conflicts among the males for the possession of the females. If the species in which this happens is monogamous in its tendencies, these jealousies and conflicts are almost certain to disrupt the larger group.

Thus we see that the family tends to oppose the formation of the larger group. The antagonism between the two has been well stated by Espinas in passages which I hesitate to translate for fear of failing to convey their exact meaning because of his idiomatic style: "L'égoïsme domestique est d'autant plus impérieux qu'il a pour centre un moi plus compréhensif et qu'il y a en lui du dévouement. La conscience collective de la peuplade ne peut donc pas avoir à sa naissance de plus grande ennemie que la conscience collective de la famille." 1 He indicates how the "peuplade" may develop and summarizes the antagonism between it and the family in the following words :-

"I. Le seul passage qu'il y ait de la famille à la peuplade se trouve non dans les relations du père avec la mère et de ceux-ci avec les jeunes, mais dans les relations des jeunes entre eux;

"2. Même à l'origine, la famille et la peuplade sont antagoniques; elles se développent en raison inverse l'une de l'autre;

"3. Le véritable élément de la peuplade est l'individu; et l'amour d'un être pour ses semblables en tant que tels, ou la sympathie, y est la source de la conscience collective." 2

This antagonism between the family and the wider form of association which we may call the peuplade is described by Petrucci also in the following words: "The family therefore is not essential to the formation of societies. The

1 Op. cit., p. 473 .

2 Des sociêtés animales, Paris, 1878, pp. 469-470. 
clan may sometimes be the extension of the family, but in certain animal species, as in man himself, it is not always the direct line of parentage which is at the basis of the group. Sometimes, furthermore, the group can be established only when the family disappears. There is antagonism between the two elements. Numerous examples of this are to be found in certain species of mammals, where the females, after being fecundated, gather by themselves, while the males form another troop elsewhere. Association exists and the family does not exist. The family, therefore, cannot be conceived as a social unity. It is not a group of families which forms Society." 1

But while it may be true that the well-developed family, such as the monogamous or polygamous family, may have stood in the way of the evolution of the larger group which we may call the horde or peuplade, still the family had prepared animals for life in this group. So that all I have said about the part played by the family in developing certain instinctive tendencies and sympathetic emotions and in laying the basis for the manifestation of intelligence holds true. The family therefore stands in the rather paradoxical position of having prepared the way for a higher form of association and then having stood in the way of its fulfillment.

Lloyd Morgan has discussed very well the part played by the family in preparing the way for the higher forms of association. He was led to discuss this by certain passages in Prince Kropotkin's well-known treatise on mutual aid. Kropotkin says ${ }^{2}$ in discussing the origin of human society

${ }^{1}$ Les origines naturelles de la propriêtê, Brussels, 1905, pp. 225-226.

2Mutual Aid a Factor of Evolution, New York, 1902, p. 79. 
that anthropology "has established beyond any doubt that mankind did not begin its life in the shape of small isolated families. Far from being a primitive form of organization, the family is a very late product of human evolution. ... Societies, bands, or tribes - not families - were the primitive form of organization of mankind and its earliest ancestors. . . . None of the higher mammals, save a few carnivores and a few undoubtedly decaying species of apes (orang-outans and gorillas), live in small families, isolatedly straggling in the woods. All others live in societies." Morgan, in commenting on this, speaks as follows: "It may at once be admitted that in all probability mankind did not have its origin in small isolated families. If we do not admit this, we must accept the alternative hypothesis, that man was developed from an unsocial ancestor. For though the biological family is the starting-point of the community, it does not of course follow that wherever there is so much coherence between parents and offspring as to form a temporary family group, a social community must in due course arise. In such unsocial carnivora as the tiger, the temporary linkage of family life is strong while it lasts. But though mankind presumably originated in a prehuman race that had already reached some degree of social coherence, there remains behind the question what was the origin of this social group? And to this question, Prince Kropotkine, in common with Darwin and Espinas, would probably answer without hesitation, that the primeval germ of the social community lay in the prolonged coherence of the group of parents and offspring. In the unsocial animals the family separates and disintegrates before the offspring mate. But if the family continue 
to cohere, the mating of offspring will give rise to the continuity of coherence found in the herd, or troop, or tribe. For new family groups will be constantly arising before the old family groups have ceased to be associated. Thus would be afforded more opportunity for tradition than among the unsocial animals." 1

\section{EMOTIONAL ForCES}

I have spoken of emotional causes of association. We have seen earlier in this book that emotions are states of feeling. As such they cannot be direct causes of behavior any more than other feelings. But we have also seen that emotions are almost always, if not always, connected with instinctive tendencies and are apparently incidental results of the manifestation of these tendencies. We have also seen that very frequently these emotions seem to influence these instinctive tendencies either by reënforcing them or by inhibiting them, thus indirectly influencing behavior. As I have said in an earlier chapter, "it sometimes happens that the emotion is strongest when the external act is inhibited, because action usually relieves the organic conditions which give rise to the emotion. This indicates how an emotion may reënforce and strengthen a tendency to an action in order to secure the relief which comes through action. This is why emotions become powerful factors in the determination of behavior." 2

Thus we see in what sense emotions may be causes of association. The emotions which are usually called sexual love and parental love reënforce the instinctive forces which bring together members of the opposite sexes and

1 Animal Behaviour, London, 1908, p. 23 I.

2 See chapter XV, p. 302. 
parents and offspring. Many other emotions are more or less indirectly forces for association, while still other emotions are forces against association. As intelligence increases, the higher animals, and especially men, discover new modes of behavior by means of which emotions can be satisfied. Like other forms of intelligent behavior, these new modes are constituted by combining inherited forms of behavior.

\section{INTELLIGENT FoRCES}

Let us now review briefly the intelligent forces at work in the family and in the peuplade, or horde, which encourage association. I have already discussed the nature of suggestion and imitation in the chapter on instinct and have indicated their social significance at various points in this book. It is probably true, as seems to be proved by experiments made by Professor Thorndike and others, that there is very little if any reflective imitation among animals other than men. But there is a certain amount of imitation which is intelligent in the sense described in a previous chapter among the higher animals and a great deal of the reflex sort which is sometimes called instinctive, but which I have shown is not instinctive in the strict sense of the word. Imitation causes uniformity of behavior and concerted action, and is therefore an important force for association. It is an effective mode of transmitting habits and other ways of doing things from one generation to another.

It must be evident that recognition plays an important part in association. The recognition may be of the reflex sort which has been described in the case of ants, where the 
recognizing is apparently done by means of odor. But whether the recognizing be done by odor, sight, touch, or sound, the animal reacts favorably to the sensations to which it is accustomed and unfavorably to those to which it is unaccustomed. So that it will harmonize with a member of its own species or social group, but will be hostile to a stranger. I have spoken as if this recognition depended upon habit, and so it does in large part. But it must be remembered that it may also have an instinctive basis to a certain extent. That is to say, the animal may inherit the tendency to react favorably or unfavorably, as the case may be, towards certain sensations.

So far I have spoken only of the recognition of the reflex sort which enables the animal to distinguish between any member of its own species or social group and a stranger. But some of the higher species also display the ability to recognize individuals. One example of this is when a pair of animals mate for a period of time or permanently. It is evident that in such a case they must distinguish each other from other members of the same species. In similar fashion parents may come to distinguish their young as individuals. But this probably happens only among the higher animals, and among them perhaps only occasionally, as has been indicated by experiments in which the offspring of a certain parent have been removed and other young of the same species or sometimes even of another species have been substituted for them without the parent apparently discovering the difference. Another example of this higher, intelligent sort of recognition is where a herd comes to recognize an individual as the leader of the herd. There is probably, among the higher animals, more or less of this recognition 
of individuals as distinguished from the mere recognition of the members of the same group or species. Both kinds of recognition are of course based upon differences in sensory stimulations, but the recognition of individuals is entirely the result of experience.

Communication also plays an important part in social relations. Speaking of the origin of communication, Lloyd Morgan says: "The foundations of intercommunication, like those of imitation, are laid in certain instinctive modes of response, which are stimulated by the acts of other animals of the same social group. These have been fostered by natural selection as a means of social linkage furthering the preservation, both of the individual and of the group." 1 As we have already seen, there are various ways of communication, such as touch, motions stimulating the vision, and sounds. But sound has proved to be the most effective means of communication for reasons which must be obvious; namely, because by means of sound communication can take place over considerable distances and because sounds can be varied to an almost infinite degree to convey different impressions and ideas. So that the ability to communicate has depended very largely upon the development of the vocal organs.

Very few of the fishes and reptiles possess the ability to make any sounds, but more of the batrachians are able to do so. The birds can make a large number of sounds, owing to the high development of their vocal organs. This is indicated by their singing and by the ability displayed by some birds to imitate human language. Probably none of the mammals except man have as highly evolved vocal organs 
as these birds. It is indeed strange that the vocal organs are no more developed among the mammals. For example, even among the anthropoid apes, who resemble man more than any other animals, the orang-utan, the chimpanzee, and the gorilla lack well-developed vocal organs. The gibbon has the best-developed organs among these apes, enabling him to utter musical sounds. And as the gibbon is the lowest of the anthropoid apes, it may be that man has inherited his vocal organs from a lower primate who was also an ancestor of these apes, and it may be that the gibbon still retains these organs in part, but that they have degenerated in the higher anthropoid apes. However, whatever the course of evolution may have been, it is evident that man has the best vocal organs, with certain birds ranking next.

But the possession of well-developed vocal organs was not sufficient to insure the appearance of the highest mode of communication; namely, language. For this to appear a high development of the cerebral hemispheres of the brain was necessary. The birds lacked this, and so failed to develop language. Whether the anthropoid apes could evolve a language if they possessed well-developed vocal organs, it is hard to say. Hence it is that communication by sounds by all animals but man is largely by means of emotional cries which do not convey any specific meaning, but which induce sympathetically similar states of feeling which lead to certain modes of behavior. Whether there is any other form of communication by sound outside of human language, it is hard to say. Certainly there is nothing in the way of the communication of abstract concepts, but it may be that some of the higher species use a few sounds which have definite meanings. 
By means of communication and of imitation there is transmitted from one generation to another what is usually called tradition; that is to say, modes of behavior and sometimes, possibly even among animals other than man, information as to conditions and events. It is evident that the amount of tradition which can be transmitted will depend upon the strength of the tendency to imitate and the ability to communicate. Furthermore, it is evident that the extent to which the benefits of experience and learning can be conserved and utilized will depend upon the extent to which tradition is transmitted. So that in this respect, also, man has the advantage over all other animals partly because of his great imitative capacities, not only in the form of organic imitation, but also to a high degree in the form of intelligent and to a much lesser degree in the form of reflective or rational imitation. But his principal advantage lies in his ability to communicate by means of language. It is frequently said with a large measure of truth that language is the principal distinction between man and the other animals, and this is so largely because by means of language man can conserve and transmit a much larger part of his experience than can any other animal without it.

There are a few phenomena connected with the horde which I wish now to discuss briefly, since they are apparently due to the forces which have been described. The first is the formation of categories among certain species. That is to say, the horde is divided up in some way and the divisions are distinguished and sometimes separated from each other. The division is in general according to sex or according to age. Certain kinds of fish segregate according 


\section{2 The Science of Human Behavior}

to sex and sometimes according to age. ${ }^{1}$ It is said that roaches when they ascend rivers are separated into groups which are composed alternately of males and of females. It has been observed that when the salmon ascend rivers, the females go before the males, with the older individuals at the head of the troop and the younger ones behind. It is said that certain kind of eels that move from one pond or lake to another are often found segregated according to sex in different bodies of water.

This formation of categories is to be found among certain birds. Perhaps the most striking case is that of the penguin. Flocks of these birds have definite places of abode where this segregation takes place. Here the flock separates into the young, the male adults, the female adults that are hatching eggs, and those that are not doing so. This formation of categories is also to be found among certain mammals. The North American bison were usually found in herds of males and herds of females with their young. Among the cetaceans the narwhals are said to live in herds of fifteen to twenty individuals which are always of the same sex. In certain herbivorous species, among which are to be found species of sheep, goats, horses, etc., at the season of the rut each of the older males gathers several females around him and then keeps the young males away from this family group. The young males then form groups by themselves. Among the primates it has been observed that when a troop of gibbons is traveling, the young are carried, the males by adult males and the females by their mothers. Among the gorillas troops of about five young gorillas are found together, but, as has been stated, these

1 Cf. R. Petrucci, Origine polyphylétique des sociétes animales, Part III, chap. 2. 
troops are broken up when they become old enough to pair. A considerable diversity of phenomena is to be found under the head of the formation of categories, but $I$ have not the space to describe them fully here. I wish, however, to discuss briefly the causes of these phenomena.

Petrucci states his theory as to the cause of these phenomena in the following passage, which I will not attempt to translate on account of its idiomatic style: "Il est facile de voir que la formation des categories, qui s'applique aux jeunes ou aux adultes, surgit ici comme une modalité particulière de la tendance associative au sein du troupeau qui ne constitue une modalité générale. C'est la recherche des semblables. On va voir qu'elle peut prendre un caractère plus général." $1 \mathrm{He}$ therefore attributes these phenomena to an associative tendency by which he seems to mean a gregarious instinct. I have already discussed the reasons for questioning the existence of a gregarious instinct. For my part, I do not know how to explain these phenomena. And indeed Petrucci himself confesses ${ }^{2}$ his inability to explain certain of these phenomena which appear among men. It is quite possible, however, that, as he suggests, the greater degree of similarity between the members of the same sex may have something to do with it, and the same would be true as between the young and adults. Furthermore, there seems to be a certain degree of antipathy between the two sexes which displays itself with considerable force even among men. This antipathy is at times overcome by the sexual instinct, but in many species this manifests itself only at the time of the rut, and at other times the sexes may be kept separate by this antipathy.

$$
1 \text { Op. cit., p. } 79 .
$$

3 Op. cit., p. 89. 


\section{The Science of Human Behavior}

Among men the rut seems to have disappeared, and the sexual instinct manifests itself with about the same strength at all seasons of the year, so that it probably acts as a restraint upon this antipathy at all times. As between the adults and the young, sexual jealousy sometimes acts as a barrier between the adults and the adolescent males. These are but a few suggestions as to the possible causes of the formation of categories. As a matter of fact, there are in all probability many causes for these phenomena, and the causes in each case vary somewhat from those in every other case. Though there are a considerable number of cases of the formation of categories in the whole animal world, and these cases appear, as we have seen, in three different classes of the vertebrates, yet the majority of the species in these classes do not display this phenomenon, while, so far as I know, it is not found at all among the amphibians and reptiles. So that it is probably due in each case to a rather peculiar combination of forces which we cannot now explain. Among primitive men it manifests itself in the separation of the sexes in men's and women's houses, in the isolation of pregnant women, etc. Magical and religious reasons are usually given for the rules enforcing these customs, and they are undoubtedly due in part to the growth of magical and religious ideas. But certain biological forces may be still more fundamental as causes. In modern society this phenomenon manifests itself in the segregation of the sexes in their club life, etc., which is largely due to certain economic, political, and social ideas and institutions, but may also be somewhat due to these biological forces.

The next phenomenon which $I$ wish to discuss is the meeting place or place of rendezvous. Many species of 
fish come to certain places each year to lay their eggs. The turtles of the Galapagos Islands lay their eggs each year at the same places on the shore. Many species of birds do the same thing. Many flocks of birds migrate each year to and fro between the same places. Many mammals have socalled rutting places where they gather each year for the males to fight for the females in their presence. Primitive men have such places of rendezvous for dances, religious ceremonies, etc., as, for example, the places where the Australian natives hold their corroborees. These meeting places appear usually to be connected with the function of reproduction. They are probably used the first time either by accident, or because they happen to be favorably located or well suited for certain purposes. Then the custom of using the same place becomes established and is transmitted by tradition from one generation to another. The existence of the place of rendezvous is then an indication of social life, and, though so frequently connected with reproduction, is probably always indicative of a social life broader than that of the family. Furthermore, it marks one of the starting points for the evolution of the right of property, for these animals display a proprietary feeling with regard to these places and drive away all intruders.

I wish to speak next of the phenomenon of leadership which sometimes plays an important part in the life of the horde. Wherever leadership exists it acts as a powerful force to bind together the members of a social group. The domination of the young by their parents may seem a case of leadership, but it is a little different from leadership in the horde, since it is due to the weakness and helplessness of the 
young during infancy. Leadership in the horde may, however, be due to superior strength, since in many species the leadership is determined by many combats among the males. In other cases the leadership may be attained as a result of initiativeness and originality. Frequently the leader is an old male, but he is not necessarily the leader because he is old, but because after having won the leadership when younger he has retained it through custom and tradition. Cases have been observed where an old female was the leader of a herd or pack. I do not know whether in these cases the position had been won by physical strength or as a result of the possession of certain mental qualities. It is doubtful if a young female is ever the leader of an animal group. But it is a physiological fact that in all the higher species the old female acquires some of the characteristics of the male. These characteristics make it possible for her to become the leader. The younger female is hampered by her reproductive functions from playing this part, as well as lacking the necessary physical and mental characteristics. These phenomena of leadership display themselves among the young at play among primitive peoples. Most of them appear among civilized men as well, but under somewhat different forms.

\section{Theories of Social Evolution}

I have now completed this brief comparative study of the fundamental types of association and their origins. Though it has been a wholly inadequate treatment of this great subject, it has, I believe, to say the least, revealed the complexity of the forces which have been at work in social evolution. I wish, therefore, before ending this chapter to 
discuss briefly and critically some of the theories of the origin and nature of society which have been formulated. This discussion will show that each of these theories has been based upon only one of the groups of forces which have been at work in social evolution, and has therefore been inadequate.

The first theory I refer to is that of the instinctive origin and nature of society. As might be expected, this theory seems to be held by most of those who believe in the existence of a gregarious instinct. Two recent representatives of this theory are McDougall and Petrucci. McDougall, while he may not expressly avow this theory at any one point, seems to be trying in his recent treatise on social psychology to explain human society entirely on the basis of instinct and of emotion, which he regards as the affective aspect of instinct. Petrucci also may not expressly avow this theory. But, as we have seen, he believes in the existence of an associative tendency, by which he seems to mean a gregarious instinct, and his constant effort is to interpret as far as possible all social phenomena in terms of this tendency.

We have seen the important part played by several instincts in social evolution. The family is perhaps wholly instinctive in its origin. But the horde certainly is not. Or even if we were to admit that it was, its evolution has depended upon the introduction and constantly increasing importance of certain intelligent forces, such as imitation, recognition, communication, leadership, etc. And the horde is, in the long run, of most importance for social evolution, for the family is by its nature necessarily a social group very limited in its numbers and very exclusive in its character. It is only in the larger size, greater diversity, and $2 \mathrm{E}$. 


\section{The Science of Human Behavior}

broader interests of the larger social groups that we can hope to find the higher stages of social evolution, and here we find intelligence playing a constantly increasing part. So that it is indeed surprising to find the highest form of society, namely, human society, interpreted so exclusively in terms of instinct.

The next theory is that of the emotional origin of society. Most of those who have held this theory have contended that society originated from sympathy. An older representative of this theory was Adam Smith, who, however, wavered somewhat between this theory and the theory that society originated from coöperation or mutual aid. Perhaps the most elaborate statement of the theory has been made by Sutherland in his treatise on the Origin and Growth of the Moral Instinct. In this voluminous work he endeavors to prove with a wealth of illustration that all social phenomena are the outgrowth of sympathetic emotions which arise from parental care. The extreme to which he carries this theory can best be indicated by means of a few quotations. For example, he speaks as follows of the part played by sympathy: "It will be subsequently perceived that parental sympathy is the basis of all other sympathy, and that sympathy in general is the ultimate basis of all moral feeling." 1 And again he says: "The sympathetic type is thus the one which is more and more distinctly emergent as we ascend in the animal scale; for not only does an increasing parental care give to a species some preference over competitive types; but an increasing conjugal stability also allies itself with this parental care, to form the home circle, and to build up the family wherein,

1 Vol. I, p. 156. 
as we shall see, is the birthplace of all moral relations." 1 Earlier in this chapter I have shown quite fully the part played by parental care in social evolution, so that I cannot be accused of ignoring its importance. But this chapter has also shown that the family could not possibly be "the birthplace of all moral relations," as Sutherland says, and by the word "moral" here he evidently means practically the same thing as "social." On the contrary, certain moral and social relations could arise only in the larger social groups, and the family is positively antagonistic to some of these relations. Indeed, we have seen that the higher types of society cannot evolve from the family, which is too narrow and exclusive in its character, but must arise from these wider forms of association.

Those who hold this theory that society can be accounted for entirely on the basis of sympathy usually have little or nothing to say about the part played by instinct. It is true that Sutherland calls his book a treatise on the moral instinct. But he scarcely mentions instinct throughout the book, and in his labored exposition of the nature of emotion gives no suggestion that the two are related to each other. And yet we have seen in earlier chapters that some emotions at any rate are caused by instinctive actions and that all emotions result either from some form of behavior or from internal physiological processes. So that it is impossible to discuss emotion apart from behavior, and if the emotions which play a part in association are caused by instincts, then the emotional theory of the origin of society could be assimilated with the instinctive theory, for the instincts would be the ultimate causes of association.

1 Vol. I, p. 29 r. 
Let us now turn to the so-called intellectualistic theories of the nature of society which give a large place to intelligence in the higher forms of association. First, I will speak of Giddings' theory of the consciousness of kind as "the original and elementary subjective fact in society." He defines this as "a state of consciousness in which any being, whether low or high in the scale of life, recognizes another conscious being as of like kind with itself." 1 If then this state of consciousness is a form of recognition, it must be an intelligent factor and his theory an intellectualistic one. However, on the very next page he remarks that "in its widest extension the consciousness of kind marks off the animate from the inanimate," which remark seems to intimate that it is coextensive with animate matter, in which case it certainly is not an intelligent factor, since, as we have seen, intelligence is not coextensive with all animate matter. However, he may not intend to imply this, for in the preface to the third edition he states that his theory was derived in part from Adam Smith's theory that society originated from sympathy, which was set forth in Smith's Theory of Moral Sentiments, but that his theory includes an element of perception. Thus he says: "I could not adopt Adam Smith's word 'sympathy,' or the familiar term 'fellowfeeling,' as a name for the primary social phenomenon, because it was necessary to recognize the element of perception. . . . The consciousness of kind, then, as I conceive it, is at once perception and feeling." Giddings' theory therefore recognizes an emotional factor and an intelligent factor, with nothing said as to instinct.

Another theory is that society originates from mutual

1 F. H. Giddings, The Principles of Sociology, New York, 1909, p. I7. 
aid. Adam Smith inclined towards this theory in his Wealth of Nations, though, as we have seen, elsewhere he inclined towards the sympathetic theory. Kropotkin has emphasized the importance of mutual aid in his treatise on this subject, in which he contends that it has been the most important factor not only in social evolution, but also apparently he would have us think in the whole of organic evolution. It is evident that conscious mutual aid as distinguished from the unconscious physiological division of labor which results from polymorphism is a highly intelligent phenomenon and does not appear until long after the beginning of social evolution.

There are various other intellectualistic theories, such as Tarde's theory that imitation has been the principal factor in social evolution, and Durkheim's theory that the influence of the many upon the individual has been the principal factor. But I believe that I have illustrated sufficiently the different types of theories and have not the space to discuss more of them. ${ }^{1}$ All of the writers whose theories have been mentioned above-namely, McDougall, Petrucci, Adam Smith, Sutherland, Giddings, Kropotkin, Tarde, and Durkheim, - have made important contributions to the analysis of social evolution, but it is evident that all of their theories are too unilateral and do not include all the factors in social evolution.

II have not discussed the theory of social evolution of the dean of American sociologists, Lester F. Ward, because it is difficult to classify his theory. He seems to regard feeling as the principal factor in social evolution, though he gives much weight to intellect also, but has little to say of instinct. (See his Pure Sociology, New York, rgo3, especially chapters XII and XV, and Psychic Factors of Civilization, Boston, 1893.) 


\section{CHAPTER XXI}

\section{CONCLUSION}

Summary of the preceding chapters, 422. - This book furnishes a basis for the study of the more complex human mental and social phenomena, 424 .

WE have now completed this survey of the evolution of behavior. The treatment has necessarily been brief, and a vast amount of data bearing on all the phenomena discussed has been omitted. But the attempt has been made to describe the principal stages in the evolution of behavior and the principal types of behavior. Let us review briefly the subjects discussed.

In all study of behavior it is necessary to begin with the structural form upon which is based the action-system which determines the behavior. The first part of the book was therefore devoted to the morphological evolution in the course of which were evolved the structural forms which determine behavior. Then were studied the direct reactions of the lower animals to external forces. But when the nervous system developed, these reactions became more or less indirect, so that we find new types of behavior appearing. The fundamental type of behavior determined by the nervous system is the reflex action. These actions become in course of time combined into complex forms which are usually called instincts. Because of the complex character of many of these instincts and the difficulty of analyzing their mechanism, there has been a tendency on the part of 
many writers in the past, and the same is still true even to-day on the part of some, to regard instinct as a form of behavior which is not mechanically determined. The attempt has therefore been made in this book to render the conception of instinct more precise than it has yet been stated by any other writer on the subject.

We then pass to intelligent behavior, the appearance of which marks a new stage in the evolution of behavior. The previous forms of behavior are inherited in the sense that animals are predetermined to manifest them when the appropriate stimuli are applied. But intelligent behavior is not inherited in this sense, but is determined by individual experience. A structural form which is capable of benefiting by experience must be inherited if intelligent behavior is to make its appearance, but the form it is to take will depend largely upon individual experience. As in the case of instinct, the attempt has been made in this book to make the conception of intelligence more precise than it has hitherto been stated by other writers on the subject. Consciousness and mind are also discussed, but it is still very difficult to make very precise our conceptions of these phenomena. They must, however, be discussed in such a book as this, because of the part they play in the determination of behavior and also because it is possible that they can be reduced to a certain extent, if not entirely, to terms of behavior. In the latter part of the book are discussed the nature and evolution of social behavior in which we find combined in the most complex fashion the types of behavior already discussed.

It has been impossible within the limits of this book even to touch upon many complex phenomena which have 


\section{The Science of Human Behavior}

evolved in the course of human social evolution and which either are forms of behavior or play an important part in determining human behavior. Among these are æsthetic, moral, religious, economic, and political phenomena. But I believe that the necessary basis for the study and analysis of these phenomena has been furnished in large part, for these phenomena are made up of the fundamental types of behavior and of mental phenomena which have been described in this book. Much writing on these subjects has been of little or no value because the writers have not had this basis. This book, therefore, furnishes a basis for the study of human mental and social evolution and also for the study of society as it now exists. 


\section{PARTIAL BIBLIOGRAPHY}

Allen, J., 1904. "The Associative Processes of the Guinea-Pig," Jour. Comp. Neur. and Psych., Vol. XIV, p. 293.

ANgell, JAS. R., I908: Psychology, 4th edit., New York.

— I9II. "Imageless Thought," Psychological Review, September, I9II.

Bain, Alexander, 1894. The Sensations and the Intellect, London. - 1899. The Emotions and the Will, London.

BaLDWIN, J. MARK, 1894. Mental Development: Methods and Processes, New York.

— (Editor), rgor. Dictionary of Philosophy and Psychology, New York.

- I902. Development and Evolution, New York.

Barker, L. F., I899. The Nervous System, New York.

Bateson, W., I894. Materials for the Study of Variation, London.

- I909. Mendel's Principles of Heredity, Cambridge.

Bell, J. C., I9o6. "The Reactions of the Crayfish," Harvard Psych.

Studies, Vol. II, p. 6I5.

- I906. "The Reactions of the Crayfish to Chemical Stimuli," Jour. Comp. Neur. and Psych., Vol. XVI, p. 299.

van Beneden, P. J., I899. Animal Parasites and Messmates, New York.

Bentley, I. M., I899. "The Memory Image and its Qualitative Fidelity," Am. Jour. of Psych., Vol. XI, p. I.

Bernard, Henry M., I9ri. Some Neglected Factors in Evolution, an Essay in Constructive Biology, New York.

Bernard, L. L., I9ro. "The Transition to an Objective Standard of Social Control," Am. Jour. of Sociology, Vol. XVI, Nos. 2 and 3 (Sept. and Nov. Igro).

BERRY, C. S., I906. "The Imitative Tendencies of White Rats," Jour. Comp. Neur. and Psych., Vol. XVI, p. 333.

- 1908. "An Experimental Study of Imitation in Cats," Jour. Comp. Neur. and Psych., Vol. XVIII, p. I. 


\section{6 The Science of Human Behavior}

Bethe, A., 1898. "Dürfen wir den Ameisen und Bienen psychische Qualitaten zuschreiben?" Pfiiger's Archiv. Bd. LXX, S. 15.

Binet, Alfred, I891. La vie psychique des micro-organismes, 2d edit., Paris.

Bohn, Georges, I906. "Les tropismes, les reflexes, et l'intelligence," L'année psychologique, edited by A. Binet, Paris.

ı 1о. La naissance de l'intelligence, Paris.

- I9Ir. La nouvelle psychologie animale, Paris.

Bolton, J. S., I9Io. "A Contribution to the Localization of Cerebral Function, Based on the Clinico-Pathological Study of Mental Disease," Brain, Vol. XXXIII, Part I 29.

Bolron, T. L., I902. " A Biological View of Perception," Psychological Review, Vol. IX, No. 6 (Nov. 1902).

Boodin, John E., 1906. "Mind as Instinct," Psychological Review, Vol. XIII, No. 2 (March, I906).

BreHM, A. E., I876-I879. Illustriertes Thierleben, 10 Bde., Leipsic. Buttel-Reepen, H. von, I900. "Sind die Bienen Reflex-maschine?"

Biol. Cent., Bd. XX, S. 97, 177, 209.

Calkins, Mary W., I905. "The Limits of Genetic and of Comparative Psychology," Brit. Jour. of Psych., Vol. I, Part 3 (Jan. 1905).

— I906. "A Reconciliation between Structural and Functional Psychology," Psychological Review, Vol. XIII, No. 2 (March, 1906). CARR, H., and WATSON, J. B., 1908. "Orientation in the White Rat," Jour. Comp. Neur. and Psych., Vol. XVIII, p. 27.

Claparède, Edouard, I903. "The Consciousness of Animals," International Quarterly, Vol. VIII (Dec.-March 1903-1904), pp. 296-315. Translated from the Revue Philosophique, Vol. LI (Mai, r9or).

- 1906. "La psychologie comparee, est-elle legitime?" Archives de psychologie, Vol. V, pp. 13-35.

CoLE, L. J., 1907. "Influence of Direction vs. Intensity of Light in Determining the Phototropic Responses of Organisms." Abstract in the Jour. Comp. Neur. and Psych., Vol. XVII, p. I93.

- 1907. "Concerning the Intelligence of Raccoons," Jour. Comp. Neur. and Psych., Vol. XVII, p. 2 II.

Cunningham, D. J., 1901. "Presidential Address before the Anthropological Section of the British Association," Nature, September 26, 1901. 


\section{Partial Bibliography}

Darwin, Charles, I859. The Origin of Species by Means of Natural Selection or the Preservation of Favoured Races in the Struggle for Life, London.

1868. The Variation of Animals and Plants under Domestication, London.

- I87I. The Descent of Man, London.

DAVENPORT, C. B., I897-r899. Experimental Morphology, 2 vols., New York.

- and Cannon, W. B., I897. "On the Determination of the Direction and Rate of Movement of Organisms by Light," Jour. Physiol., Vol. XXI, p. 22.

and Lewrs, F. L., I899. "Phototaxis of Daphnia," Science, N. S., Vol. IX, p. 368.

and Perkins, H., I897. "A Contribution to the Study"of Geotaxis in the Higher Animals," Jour. Physiol., Vol. XXII, p. 99.

Dearborn, G. V. N., Igoo. "The Individual Psycho-Physiology of the Crayfish," Am. Jour. Physiol., Vol. III, p. 404.

Deniker, J., Igoo. The Races of Man, London.

DurkheIm, EMrLe, I902. De la division du travail social, 2d edit., Paris.

Edinger, Ludwig, I899. "Haben die Fische ein Gedachtniss?" Allgemeine Zeitung, Beilage, October $2 \mathrm{I}$ and 23. (Translation in Smithsonian Report, I899, p. 375.)

- I908. "The Relations of Comparative Anatomy to Comparative Psychology," Jour. of Comp. Neur. and Psych., Vol. XVIII, No. 5 (Nov. I908).

Ermer, Th., I888. Die Entstehung der Arten auf Grund von Vererbung erworbener Eigenschaften nach den Gesetzen organischen Wachsens, Jena.

1898 . On Orthogenesis and the Impotence of Natural Selection, Chicago.

Enteman, M. M., I902. "On the Behavior of Social Wasps," Pop. Sci. Mo:, Vol. LXI, p. 339.

Espinas, Alfred, I878. Des sociétés animales, Paris.

FABRE, J. H., I878-I904. Souvenirs entomologiques, 9 vols., Paris. FERrIER, David, I878. The Localization of Cerebral Diseases, London.

I886. The Functions of the Brain, New York. 
Frelde, Adele M., I904. "The Power of Recognition among Ants," Biol. Bul., Vol. VII, p. 227 .

- 1905. "The Progressive Odor of Ants," Biol. Bul., Vol. X, p. I. Forsom, J. W., Igo6. Entomology, Philadelphia.

FOREL, A., I874. Les fourmis de la Suisse, Zurich.

- I904. Ants and some Other Insects, translated by W. M. Wheeler, Chicago.

- I908. The Senses of Insects, London.

Fournier, D'Albe, E. E., Igo6. The Electron Theory, London.

Franz, S. I., "On the Functions of the Cerebrum: The Frontal Lobes," Archives of Psychology, Vol. I.

- I910. "On the Association Functions of the Cerebrum," Jour. of Phil. Psych. and Sci. Meth., Vol. VII, p. 679.

- Igri. "The Functions of the Cerebrum," Psychological Bulletin, Vol. VIII, No. 4.

- Igr2. "The New Phrenology," Science, N. S., Vol. XXXV, p. 321.

Galton, Francis, 1883. Inquiries into Human Faculty and its Development, London.

- r889. Natural Inheritance, London.

Giddrngs, F. H., rgog. The Principles of Sociology, New York.

GoldsMith, M., I905. "Récherches sur la psychologie de quelques poissons littoraux," Bull. Inst. gen. psych., Paris, tome V, p. $5 \mathrm{I}$. GolTz, F., I892. "Der Hund ohne Grosshirn," Pflüger's Archiv, Bd. LI. Groos, KarL, 1898. The Play of Animals, New York. Translated from the German.

- rgor. The Play of Man, New York. Translated from the German.

Hachet-Souplet, P., 1900. Examen psychologique des animaux, Paris.

Haggerty, M. E., rgog. "Imitation in Monkeys," Jour. Comp. Neur. and Psych., Vol. XIX, p. 337.

- I909. "Imitation in Monkeys," Century Mag., p. 544.

Herrick, C. J., Igro. "The Evolution of Intelligence," Science, Vol. XXXI.

HerRICK, C. L., I904. " The Beginnings of Social Reactions in Man and Lower Animals," Jour. of Comp. Neur. and Psych., Vol. XIV, No. 2, pp. II8-r23. 


\section{Partial Bibliography}

HILl, Alex., 1908. The Body at Work, London.

Hoвнouse, L. T., Igor. Mind in Evolution, London.

Hodge, C. F., and Arkins, H. A., I895. "The Daily Life of a Protozoan," Am. Jour. Psych., Vol. VI, p. 524.

Holleman, A. F., 1903. A Textbook of Organic Chemistry, New

York. Translated from the second Dutch edition by A. J. Walker.

Holmes, S. J., 1905. "The Selection of Random Movements as a Factor in Phototaxis," Jour. Comp. Neur. and Psych., Vol. XV. pp. 98-II2.

- Igr. "The Beginnings of Intelligence," Science, Vol. XXXIII, No. 848 (Mar. 3I, I9II), pp. 473-480.

- I9Ir. The Evolution of Animal Intelligence, New York.

Hort, E. B., and LeE, F. S., rgor. "The Theory of Phototactic

Response," Am. Jour. of Physiology, Vol. IV, pp. 460-48I.

HousSAY, FrÉDÉRIC, I893. The Industries of Animals, London.

Howell, W. H., I906. A Textbook of Physiology, Philadelphia.

Hudson, W. H., I895. The Naturalist in La Plata, London.

JAMES, William, igo6. "La notion de conscience," Archives de psychologie, Vol. V, pp. I-I 2.

- 1896. Principles of Psychology, New York, 2 vols.

JANET, C., I893-I905. Les fourmis, les guêpes et les abeilles, Paris. Jennings, H. S., I899. " "The Psychology of a Protozoan," Am. Jour.

Psych., Vol. X, p. 503.

- 1904. Jour. Comp. Neur. and Psych., Vol. XIV, pp. 464-468.

- I906. Behavior of the Lower Organisms, New York.

- I908. "The Interpretation of the Behavior of the Lower Organisms," Science, Vol. XXVII, p. 705.

- 1910. "Diverse Ideals and Divergent Conclusions in the Study of Behavior in Lower Organisms," Am. Jour. Psych., Vol. XXI, pp. 349-370.

Johannsen, W., 1903. Ueber Erblichkeit in Populationen und in reinen Linien, Jena.

JoHnston, J. B., Igo6. The Nervous System of Vertebrates, Philadelphia.

Jones, Harvey C., 1906. The Electrical Nature of Matter, New York.

Judd, C. H., I9ro. "Evolution and Consciousness," Psychological Review, Vol. XVII, No. 2 (March, I9I0), pp. 77-97. 
Kellogg, V. L., 1908. Darwinism To-day, New York.

Kinnaman, A. J., 1902. "Mental Life of Two Macacus Rhesus

Monkeys in Captivity," Am. Jour. Psych., Vol. XIII, pp. 98-173. KirkPatrick, E. A., I909. Genetic Psychology, New York.

von Krafft-Ebing, R., I905. Textbook of Insanity, Philadelphia.

Translated from the German.

Kropotkin, P., I902. Mutual Aid a Factor of Evolution, New York.

Lamarck, J. B., 1909. Philosophie zoölogique, Paris.

LANE, C. C., 1907. "The Early Surroundings of Life," Science,

Vol. XXVI, pp. I 29-I43.

LARMOR, JOSEPH, I900. Æther and Matter, Cambridge.

Le Dantec, F., I906. Traité de biologie, Paris, 2d edit.

LEwes, G. H., 1874-1880. Problems of Life and Mind, London.

Lock, R. H., I9I0. Recent Progress in the Study of Variation,

Heredity, and Evolution, 2d edit., New York.

LOdge, Oliver, I903. Modern Views of Matter, Oxford.

LOEB, JACQUES, I900. Comparative Physiology of the Brain and Comparative Psychology, New York.

— 1905. Studies in General Physiology, 2 vols., Chicago.

— 1906. The Dynamics of Living Matter, New York.

- 1907. "Concerning the Theory of Tropisms," Jour. of Experimental Zoölogy, Vol. IV., pp. I 51-I58, Philadelphia.

- 1909. "Experimental Study of the Influence of Environment on Animals," in Darwin and Modern Science, edited by A. C. Seward, Cambridge.

Luвbоcк, J., I888. On the Senses, Instincts, and Intelligence of Animals, with Special Reference to Insects, New York.

- 1892. Ants, Bees, and Wasps, New York.

Lukas, F., 1905. Psychologie der niedersten Thiere, Vienna and Leipzig.

Lyon, E. P., 1904. "On Rheotropism, I. Rheotropism in Fishes," Am. Jour. Physiol., Vol. XII, p. I49.

McCabe, Joseph. Evolution, New York.

- 1910. The Evolution of Mind, London.

Mach, E., I897. Contributions to the Analysis of the Sensations, Chicago.

McCook, H. C., I909. Ant Communities and How they Are Governed, New York. 
McDougald, William, I905. Physiological Psychology, London.

- 1909. An Introduction to Social Psychology, Boston.

- IgIr. Body and Mind, A History and a Defense of Animism, New York.

Marshall, H. R., I894. Pleasure, Pain and Esthetics, New York. - I898. Instinct and Reason, New York.

Mast, S. O., I9Ir. Light and the Behavior of Organisms, New York.

MeYer, Adolf, I898. "Critical Review of the Data and General Methods and Deductions of Modern Neurology," Jour. Comp. Neur., Vol. VIII.

von MEYer, ERNSt, 1891. A History of Chemistry, London. Translated from the German.

Meyer, Max, 1908. "The Neural Correlate of Pleasantness and Unpleasantness," Psychological Review, Vol. XV, No. 5 (Sept. 1908).

Mrrus, T. W., 1894-1896. The Psychic Development of Young Animals and its Psychical Correlation, Montreal.

- 1898 . The Nature and Development of Animal Intelligence, New York.

Mınot, C. S., I902. "The Problem of Consciousness in its Biological Aspects," Science, Vol. XVI, No. 392 (July 4, 1902).

Montague, Wm. P., Igo8. "Consciousness as Energy," in Essays Philosophical and Psychological in Honor of William James, New York.

MoRgAN, C. LLOYD, I894. Introduction to Comparative Psychology, London.

- 1895. Animal Life and Intelligence, Boston.

- I896. Habit and Instinct, London.

— 1908. Animal Behaviour, 2d edit., London.

- I9ro. "Instinct and Intelligence," British Journal of Psychology, Vol. III, Part 3, pp. 219-229.

MoRgan, T. H., Igo3. Evolution and Adaptation, New York.

- I907. Experimental Zoölogy, New York.

Murr, M. M. Patrison, 1907. A History of Chemical Theories and Laws, New York.

MuMford, Eben, I906-1907. "The Origins of Leadership," $A m$. Jour. of Sociology, Vol. XII, pp. 216-240, 367-397, 500-53r.

Munk, H., I88I. Ueber die Functionen der Grosshirnrinde, Berlin. 
voN NÄGeli, CARL, I884. Mechanisch-Physiologische Theorie der Abstammungslehre, Munich and Leipzig.

- I898. A Mechanico-Physiological Theory of Organic Evolution, Chicago.

Norman, W. W., I899. "Do the Reactions of Lower Animals Due to Injury Indicate Pain Sensations?" in Woods Holl Biological Lectures, Boston.

- I900. "Do the Reactions of the Lower Animals Against Injury Indicate Pain Sensations?" Am. Jour. of Physiology, Vol. III, No. 6 (Jan. I900).

Nuel, J. P., I904. La vision, Paris.

—— I905. "La psychologie comparee est-elle legitime? Réponse à M. Claparède," Arch. de psych., tome V, p. 326.

Parker, G. H., I909. "The Origin of the Nervous System and its Appropriation of Effectors," in the Pop. Sci. Mo., Vol. LXXV, pp. 56-64, I37-r45, 253-263, 338-345.

Pearson, KarL, igio. Biometrika, Vol. VI, pp. 348-353.

Peckham, Geo. W., and Elizabeth G., I905. Wasps Social and Solitary, Boston.

Perrier, E., Colonies animales, Paris.

Petrucci, R., I905. Les origines naturelles de la propriété, Brussels.

— I906. Origine polyphyletique, homotypie et non comparabilité directe des sociétés animales, Brussels.

Pierce, A. H., igo6. "An Appeal from the Prevailing Doctrine of a Detached Subconsciousness," in Studies in Philosophy and Psychology, dedicated to C. E. Garman, Boston.

Pike, F. H., I9I2. "A Defence of the 'New Phrenology," Science, N. S., Vol. XXXV, pp. 619-622.

Porter, JaMres P., Igro. "Intelligence and Imitation in Birds," Am. Jour. of Psychology, Vol. XXI, No. I (Jan. 19Io).

Punnetr, R. C., IgIr. Mendelism, New York.

Read, Carveth, IgII. "Instinct Especially in Solitary Wasps," British Journal of Psychology, Vol. IV, Part I (May, I9I I). Richet, Charles, i887. Essai de psychologie générale, Paris. Romanes, G. J., Journal of the Linnean Society, Vol. XIX, pp. 337-4II.

- I892. Animal Intelligence, 5th edit., London.

I898. Mental Evolution in Animals, New York. 
Rouse, J. E., Igo6. "The Mental Life of the Domestic Pigeon,"

Barvard Psych. Studies, Vol. II, p. 580.

Royce, Josian, rgo4. Outlines of Psychology, New York.

RUTHERFORD, E., Igo6. Radioactive Transformations, New York.

von SACHS, J., 1887. Vorlesungen über Pflanzen-Physiologie, 2d edit., Leipzig.

SaNTEE, H. E., 1907. Anatomy of the Brain and Spinal Cord, Philadelphia.

Schaefrer, A. A., Igro. "Selection of Food in Stentor," Jour. Exp. Zool., Vol. VIII, p. 75.

- Igrr. "Habit Formation in Frogs," Jour. of Animal Behavior, Vol. I, p. 309 .

SHEPHERD, W., Igro. "Some Mental Processes of the Rhesus Monkey," Psych. Rev. Monograph Supp., Vol. XII, No. 52.

SHERRIngton, C. S., Igo6. The Integrative Action of the Nervous System, New York.

- IgIr. "The Rôle of Reflex Inhibition," Science Progress, No. 20.

Small, W. S., 1899. "Notes on the Psychic Development of the Young White Rat," Am. Jour. Psych., Vol. XI, p. 80.

- r899-I900. "An Experimental Study of the Mental Processes of the Rat," Am. Jour. Psych., Vol. XI, p. 133, Vol. XII, p. 206. Sirth, Adam, I759. The Theory of Moral Sentiments, London.

- I776. An Inquiry into the Nature and Causes of the Wealth of Nations, 2 vols., London.

Smtth, Stevenson, rgo8. "The Limits of Educability in Paramœcium," Jour. Comp. Neur. and Psych., Vol. XVII, p. 499.

SNYDER, CarL, I909. "The Physical Conditions at the Beginning of Life," Science Progress, Vol. III, pp. 579-596.

Spaulding, E. G., I904. "An Establishment of Association in Hermit Crabs, Eupagurus longicarpus," Jour. Comp. Neur. and Psych., Vol. XIV, p. 49.

Spencer, Herbert, 1895. Principles of Psychology, $3 \mathrm{~d}$ edit., New York.

- I900. The Principles of Biology, 2 vols., New York.

StRASBURGer, E., I878. Wirkung des Lichtes und der Wärme auf Schwarmsporen, Jena.

Sutherland, AleXander, I898. The Origin and Growth of the Moral Instinct, 2 vols., London. 
Tarde, GabrIel, I900. Les lois de l'imitation, 3 d edit., Paris. Thomson, J. A., rgo6. The Study of Animal Life, New York.

- I909. Heredity, New York.

- I909. Darwinism and Human Life, New York.

Tromson, J. J., I906. Conduction of Electricity through Gases, 2d edit., Cambridge.

- 1907. The Corpuscular Theory of Matter, London.

ThORNDIKe, Edward L., IgIr. Animal Intelligence, New York.

Titchener, E. B., I902. "Were the Earliest Organic Movements Conscious or Unconscious?" Pop. Sci. Mo., Vol. LX (March, I902), pp. $35^{8-369 .}$

- I909. Lectures on the Experimental Psychology of the Thought Processes, New York.

Torrey, H. B., I907. "The Method of Trial and the Tropism Hypothesis," Science, Vol. XXVI, p. 3 I 7.

Triplett, N. B., I90I. "The Educability of the Perch," Am. Jour. Psych., Vol. XII, p. 354 .

Turner, C. H., I906. "A Preliminary Note on Ant Behavior," Biol. Bul., Vol. XII, p. 3г.

- I907. "The Homing of Ants," Jour. Comp. Neur. and Psych., Vol. XVII, p. 367 .

Veblen, Thorstein, I898. "The Instinct of Workmanship and the Irksomeness of Labor," Am. Jour. of Sociology, Vol. IV, pp. I87-20I.

I899. The Theory of the Leisure Class, New York.

Vernon, H. M., I903. Variation in Animals and Plants, New York. Verworn, MAX, I899. Psycho-physiologische Protistenstudien, Jena. I899. General Physiology, London. Translated from the German by F. S. Lee.

DE VRIEs, Hugo, I9OI. Die Mutationstheorie, Leipzig. I905. Species and Varieties, their Origin by Mutation, Chicago.

WARD, JAmes, I904. "On the Definition of Psychology," Brit. Jour. of Psych., Vol. I, Part I (Jan. I904).

Ward, L. F., I893. Psychic Factors of Civilization, Boston.

- I903. Pure Sociology, New York.

Washburn, Margaret F., I904. "A Factor in Mental Development," Philosophical Rev., Vol. XIII, p. 622.

I908. The Animal Mind, New York. 
WASMANN, Eric, Ig03. Instinct and Intelligence in the Animal Kingdom, St. Louis. Translated from the German.

Watkins, G. P., 1900. "Psychical Life in Protozoa," Am. Jour. Psych., Vol. XI, p. 166.

Watson, J. B., I907. "Kinæsthetic and Organic Sensations: Their Roble in the Reactions of the White Rat to the Maze," Psych. Rev. Monograph Supp., Vol. XIII, No. 2.

— I908. "Imitation in Monkeys," Psych. Bul., Vol. V, p. I69.

Weismann, August, I893. The Germ Plasm, London. Translated from the German.

- I893. "The All-Sufficiency of Natural Selection," Contemporary Rev., Vol. LXVI, pp. 309 and 596.

- I896. On Germinal Selection as a Source of Definite Variation, Chicago. Translated from the German.

- 1904. The Evolution Theory, 2 vols., New York.

WheELER, W. M., Igro. Ants Their Structure, Development, and Behavior, New York.

Whetham, W. C. D., I904. The Recent Development of Physical Science, Philadelphia.

WhitMan, C. O., I899. "Animal Behavior," in Biological Lectures from the Marine Biological Laboratory, Woods Holl, Mass., Boston.

WiLDER, H. H., Igog. History of the Human Body, New York.

WrLson, E. B., Igo6. The Cell in Development and Inheritance, 2 d edit., New York.

Woodworth, R. S., Igo6. "The Cause of a Voluntary Movement," in Studies in Philosophy and Psychology, dedicated to C. E. Garman, Boston.

- 1908. "The Consciousness of Relation," in Essays Philosophical and Psychological, in Honor of William James, New York.

Wundt, W., 1904. Principles of Physiological Psychology, Vol. I, London. Translated from the German.

Yerkes, R. M., I903. "The Instincts, Habits and Reactions of the Frog. I. Associative Processes of the Green Frog," Harvard Psych. Studies, Vol. I., p. 579 .

— I905. "Animal Psychology and Criteria of the Psychic," Jour. 
of Phil. Psych. and Sci. Meth., Vol. II, No. 6 (March 16, 1905), pp. I4I-I 49.

1907. The Dancing Mouse, New York.

- and Hugcins, G. E., I903. "Habit Formation in the Crawfish, Cambarus affinis," Harvard Psych. Studies, Vol. I, p. 565.

YoAKUM, C. S., I909. "Some Experiments on the Behavior of Squirrels," Jour. Comp. Neur. and Psych., Vol. XIX, p. 54I.

ZIEgler, H. E., I9Io. Der Begriff des Instinktes einst und jetzt, Jena. 


\section{INDEX}

\section{A}

Acquisitiveness, 242.

Action system, the, 26 ff., 269 .

Adaptation, 26, I46, 213 ff., 232 ff., 263.

Affection, 244, 304 .

Allelomorph, 55 ff., 6r, 67.

Allotropism, 13.

Amoba, I 8 ff.

Amphibian, 1 57, $370 \mathrm{ff}$.

Amphimixis, 43 .

Amphioxus, 329.

Anemotropism, $\mathrm{Ir} 6$.

Angell, J. R., 278, 313.

Anger, 302.

Ant, 106, 268, $338 \mathrm{ff}$.

Anthropoidea, 384 ff.

Anthropology, 72 ff., $x 72$.

Ape, 280, 309, 333 ff., 357,385 ff.

Aphid, 349 ff.

Arachnida, 35x.

Arm, 270 ff., 272.

Arthropoda, 35x.

Aspredo, 367 .

Association, 327 ff., 355, 364 ff., $390 \mathrm{ff}$.

Association area, I8I ff., $211,265,270 \mathrm{ff}$., $272,274,280,288,290,359$ ff.

Associative memory, 260 ff., 288.

Atom, 8.

Attention, $290 \mathrm{ff}$.

Aye-Aye, 384 ff.

\section{B}

Baboon, 385 .

Bacteria, $120 \mathrm{ff}$.

Badger, 38r, 382.

Bain, A., 295.

Baldwin, J. M., r34, 225, 277, 279, 28I, 282, 295, 297.

Barker, L. F., I78.

Barotaxis, II $3 \mathrm{ff}$.

Bat, 379.

Bateson, W., 46 ff., 55 ff., 6I, 63, 7I.
Batrachia, $370 \mathrm{ff}$.

Beaver, 380 .

Bee, 268, 294, $338 \mathrm{ff}$.

Behavior, I ff., I98, 256 ff., 422 ff. animal, I, 74, $76 \mathrm{ff}$., II $7 \mathrm{ff}$. definition of, $\mathbf{I}$.

human, 3.

Bergson, H. 266 ff.

Bethe, A., 26r.

Binet, A., 80, 278, 360 .

Biogen, 37 .

Biology, 2, 68 ff., 75 ff., I72.

Biophor, 39, 53.

Bird, I68, 233, 268, 333, 345, 372 ff.

Bison, 253, 412.

Bohn, G., 82, 96, 268.

Bolton, J. S. 185 , I93.

Brain, I57, I59, I63, I66 ff., I75 ff., I95, 228 ff., 270 ff., 302 ff., 359 ff., 380 .

Brandt, 360 .

Brehm, A. E., 364 .

Brooding, 373 .

Buhler, 278.

\section{C}

Calkins, Mary W., 3I4, 319 ff.

Campbell, A. W., I84.

Carbon, 13.

Carnivora, 333, 381 ff., 391.

Cassowary, 373 .

Cat, I90, I91, 381, 382, 383 .

Categories, 4 II ff.

Caterpillar, 342.

Cebida, 385 .

Cell, the organic, I6 ff., 49, 328.

Central nervous system, I56 ff., I7 23I, 262 ff., 288, 322, 336.

Cercopithecida, 385 .

Cerebellum, I67, r68 ff., 228 ff.

Cerebral localization, $175 \mathrm{ff}$.

Cerebrum, I59, I67, I68, I72 ff., I76 ff, $228 \mathrm{ff}$., $265,270$.

Cetacea, 379.

Chain instinct, $230 \mathrm{ff}$., $399 \mathrm{ff}$. 
Characters,

acquired, 27, 30 ff., $216,217,286,337$. degeneration of, $3 \mathrm{I}, 40 \mathrm{ff}$.

Chemistry, 7.

Chemotropism, 109 ff., 335 .

Chimpanzee, 173,385 ff., 394.

Chiroptera, 379.

Chromatin, 22.

Chromosome, 66 ff.

Chromotropism, $102 \mathrm{ff}$.

Circulation, 20.

Civet, 38r.

Claparède, E., 316.

Cleanliness, 242.

Colenterata, 268.

Colony, 328 ff., $340,345,356$.

Commensalism, 342 , 351 .

Common path, the, I60 ff., 212.

Communication, $337,362,375,409$ ff.

Concept, 278.

Conduct, 2.

Conjugal relation, the, $378,399 \mathrm{ff}$.

Consciousness, $130, \mathrm{r}_{34}, 2 \mathrm{I} 4 \mathrm{ff}$., 233, 257, $260,273,275,280,28$ I ff., 322 ff.

Consciousness of kind, 420 .

Constructiveness, 242.

Contact irritability, I I 3 ff.

Coöperation, 254, 335, 363, 376.

Copulation, II4.

Corpuscle, 9 .

Correns, 55.

Corroboree, 4 I5.

Cortex, I 78 ff., I8I, I 84 ff., I96, 229, 263 ff., 270.

Cranium, 175, 271.

Crayfish, 268.

Crocodile, $37 \mathrm{r}$.

Crustacean, 268, 293, 352.

Crystals, 16, 25, 29.

Cunningham, D. J., 27I, 272

Curiosity, 242.

Cytoplasm, 22.

\section{D}

Darwin, Chas., 29, 32 ff., 40 ff., 53, 63, $65,70,238,301,393,405$.

Davenport, C. B., $58,82 \mathrm{ff} ., 84,85,90$, $92,100,102,107, I_{3}$ ff., II6, II 7 .

Deciduate, $378 \mathrm{ff}$.

Deer, 380.

Delage, 39 .

Deniker, J., 173.

Determinant, $39,40 \mathrm{ff}$.
Disease, functional, 195 ff.

organic, $195 \mathrm{ff}$.

Disgust, 302.

Division of labor, 254 .

Dog, I70, 249, 302, 309, 381, 382, 383 .

Domestication, 382 ff.

Duckbill, 377.

Dujardin, 360 .

Durkbeim, E., 421.

\section{$\mathbf{E}$}

Earthworm, 96, 105 ff.

Echidna, 377.

Echinodermala, 293.

Ectoparasite, 354 ff.

Edentata, 379.

Edinger, L., 229, 270.

Education, 273.

Eimer, T., 27 ff., 34, 45 .

Electricity, 9, II2, II9, 123.

Electrolyte, 9 .

Electron, 9.

Electrotropism, I 12.

Elephant, 380.

Emery, 46.

Emotion, 219, 220 ff., 286, 299 ff., 372 ff., 406 ff., 4 I 8 ff.

Emu, 373.

Emulation, 24I, 25I.

Energy, 7 .

Entoparasite, $354 \mathrm{ff}$.

Environment, 337, 395.

Envy, 244.

Escherich, 351 .

Espinas, A., 328, 403, 405.

Ether, 7, 99.

Euglena viridis, $96 \mathrm{ff}$.

Evolution, organic, 12, 45, 134, 136, 318,331 ff.

Experience, 210, 257 ff., 315.

Eye, 214.

\section{$\mathbf{F}$}

Fabre, J. H., 233. .

Face, $271,272$.

Facilitation, 160.

Family, 336, 340, 343 ff., 374 ff., 399 ff.

Fear, 242, 302, 304 .

Feeling, 219, 261, 295 ff., 316 ff.

Finch, 374 .

Fish, II $5,157,268,294,330,333,336$, $365 \mathrm{ff}$. 
Fission, 21 ff., 335.

Flechsig, 181, 182, 185, 270, 271.

Flight, 244 .

Flögel, 360 .

Flounder, 294.

Fly, 294, 355.

Folsom, J. W., rg9.

Forel, A., 26r, 268, 360 .

Formicida, 345, 346, 356 .

Fossil, 337, 364 .

Franz, S. I., r76, 189 fi.

Frog, 370.

\section{G}

Gall, r79.

Galton, F., 39, 46, 5r, 60 ff., 73, 380.

Galvanotropism, 112.

Gemmules, 35, 39, 53 .

Geotropism, ro 7 ff.

Germ cell, 22 ff., 35 ff., 39 ff., 53 ff.

Germ plasm, 38 ff., 34I.

Gestation, 384,387 .

Gibbon, the, 385 ff., 391 .

Giddings, F. H., 420, 421 .

Gland, I44, I 49 ff.

Goltz, F., 304 .

Gorilla, I 73, 385 ff., 39r, 394 .

Gravity, I07 ff., I 23.

Gregariousness, 252 ff., 331, 380 ff., 385, 39 I, 395 ff.

Groos, K., 250.

Gulick, 34 .

Gynandromorph, 67 .

\section{H}

Habit, 216, 2 I 7, 254 ff., 264, 286, 302.

Hæckel, E., 39, 80 .

Hand, 270 ff., 272.

Hapalida, 385 .

Harvesting ant, 347 .

Hawk, 254, 393.

Head, 270.

Heart, 202.

Heat, 116, 120, 223.

Heliotropism, see phototropism.

Helmholtz, H., 2 I4.

Herbivora, 333.

Heredity, see inheritance.

Herrick, C. J., 264, 289, 3I4, 3I5, 3 I 7 .

Heterogenesis, 24, $45 \mathrm{ff}$.

Heterozygote, 56 ff., 61 .

Hobhouse, L. T., 140, 14I, 203, 204, 2 18, $308,310,312$.
Holleman, A. F., 113 .

Holmes, S. J., 98, 26r, 268.

Holt, E. B., 94.

Hominida, 385 .

Homozygote, 56 fif., $6 \mathrm{r}$.

Horde, 387,404 ff., 4 II ff.

Horse, 380 .

Howell, W. H., I 55, I78, 202.

Hunting ant, 346 .

Hunting instinct, 242, 249, 250, 346 .

Huxley, T. H., 287.

Hybrid, 55 ff., 73.

Hydrogen, 8, I4.

Hydrotropism, I 6 .

Hymenoptera, 340, 360.

\section{I}

Id, 39 .

Idea, I 80 ff., 270, 29r, 3rr, 316 ff., 337.

Ideo-motor, 246 ff., 275 .

Image, 2 I I, 274 ff., 29I, 3 II.

Imageless thought, $278 \mathrm{ff}$.

Imitation, 2I 2, 24I, 246 ff., 25 I, 337, 355, 359,362 ff., $375,407$.

Impulsive instinct, $230 \mathrm{ff}$., $240 \mathrm{ff}$.

Infusoria, 268, 328.

Inheritance, 60 ff., 204, 208, 216, 332, 400 .

Inhibition, I60, 2 I I, 2 I2, 275 ff., 31 I ff.

Innate tendencies, 244,245 fi.

Insect, II I, 233, 268, 294, $338 \mathrm{ff}$.

Insectivora, 379 .

Instinct, I 97 ff., 266 ff., 286, 300, 395 ff., $417 \mathrm{ff}$.

a new definition of, 226 .

Integration, I6I ff., 205, 206, 209, $25 \mathrm{I}$, $263,266,308,311$ :

Intellect, see intelligence.

Intelligence, 2I 2, 229 ff., 238 ff., 256 ff., 289, 314 ff., 322 ff., $337,338,346$, 359 ff., 383,407 ff., 420 ff.

Introspection, 257, 278, 294 .

Invertebrate, 268 ff., 288, 333, 338, 363 .

Ion, 9.

Irritability, 79 ff., 138 .

Isomerism, I3.

\section{J}

Jackal, 392 .

James, W., 207, 2 Ir, 220 ff., 223 ff., 238, 240 ff., $283,300,305$.

Jealousy, 376, 382, 403, 4I4. 
Jennings, H. S., $76,77,78,83$ ff., 85, Marshall, H. R., 245. $95 \mathrm{ff}$., I04, I1 1 ff., I I 5, I 8 ff., 138 ff., $259,284 \mathrm{ff}$.

Johannsen, W., 64 .

Johnston, J. B., 162, 170, I71, 174, 178, I8I ff., I92, 27 I.

Jones, H. C., II.

Judd, C. H., $316 \mathrm{ff}$.

\section{$\mathbf{K}$}

Kellogg, V. L., 7I.

Kelvin, Lord, 37 .

Kenyon, 360 .

Knowledge, $266 \mathrm{ff}$., nature of, 4, $325 \mathrm{ff}$.

von Kölliker, 46.

Korschinsky, 46.

von Krafft-Ebing, R., I94, 195 ff.

Kropotkin, P., 404 ff., 42 I.

\section{$\mathbf{L}$}

Lamarck, J. B., 30 ff., 36,45 .

Lane, A. C., 18.

Lange, 220 ff., 300 .

Language, $409 \mathrm{ff}$.

Larva, 342 ff., 352,355 .

Leadership, 363,415 ff.

Learning, 26r, 264, 273 ff., $315,363$.

Le Dantec, F., 39, 54 .

Lee, F. S., 94 .

Lemur, $333,384 \mathrm{ff}$.

Lemuroidea, 384 ff.

Lewes, G. H., $216,286$.

Leydig, 360 .

Light, 88 ff., 1 I9.

Lion, 254 .

Lizard, 37 .

Lodge, Oliver, 10.

Loeb, J., 22, 54, 78, 8 r ff., 89 ff., 92, 96 , 100 ff., 103, 106, 108, 109 ff., I12, I38 ff., I 5 I, 198 ff., 260 ff., 268, 288, 294, 314 .

Lubbock, J., 233, 268.

\section{M}

Macaque, 385 .

Magic, 414.

Mammal, 157, 170, 268, 280, 295, 333, $345,364,372,376 \mathrm{ff}$.

Mammary glands, $377 \mathrm{ff}$., 384 .

Man, 280, 364, 385, 387 ff.
Marsupial, 333,377 ff.
Mast, S. O., 88.
Maternal care, 203, 249, 367 ff., 373, 384 ff.
Matter, characteristics of, 7, 282, 285, 287,323 ff.
organic, 7, I 2 ff., 79 ff., $245,274,285$, 287.

McCabe, J., 18, 272.

McDougall, W., I40, I60, I63, 212, 218 ff., $244,322,417,421$.

Medulla oblongata, $167,228$.

Meeting places, $414 \mathrm{ff}$.

Memory, 2I6, 3 II.

Mendel, G., 55 ff., 64, 67, 71 ff.

Metabolism, I7, 20, 100, I 21.

Metaphyta, 24.

Metazoa, 24, I 8 ff., I 27 ff., I 38, I48, 328, 335,336 .

Meyer, Max, 298, 316.

Micella, 39.

Micromerism, 39, $53 \mathrm{ff}$.

Mills, $184,185,187$.

Mind, 214 ff., 257, 28r, 286, 294, 321 ff., 328 ff., $348,355,359,363,389$.

Minot, C. S., 3 I 7 .

Mitosis, 2I, 49.

Modesty, 242.

Molecule, 8.

Mollusk, 328 .

Monism, 283.

Monkey, I90, I9I, 280.

Monogamy, 375, 387 .

Monotreme, 333 .

Montague, W. P., 314, 325.

Morgan, C. L., I34, 202, 203, 214, 224, $225,255,261,302,303,315,348$, 392,404 ff.

Morgan, T. H., 52, 53, 65, 106, 199.

Moth, ro7.

Mott, F. W., 229.

Mouth, 271, 272.

Mulatto, 58.

Multiple personality, 309 ff.

Muscle, 144, 148 ff., 202.

Mutation, $5 \mathrm{I}$ ff., $58 \mathrm{ff}$., $207,208$.

Mutual aid, 254, 42 I.

Myrmecophilism, 35I ff.

Myrmecoxene, 352.

\section{N}

Nägeli, C., 25 ff., 39, $4 \dot{5}$.

Negro, 58. 
Neo-Darwinism, 38.

Neo-Lamarckism, 28, 5 I ff.

Nerve, 137 ff., 201 ff., 227 ff., 304 ff., $316,336,384$.

Nest, 232, 233, 338, 342 ff., 347, 368.

Neuromere, 163,167 .

Neurone, ${ }_{54}$ ff., 275 ff.

Newt, 370.

Nitrogen, 14 .

Nondeciduate, $378 \mathrm{ff}$.

Norman, W. W., 292 ff.

Nuptial flight, 343 .

Enothera Lamarckiana, 5 I fi.

Opossum, 377.

Optimum, the, 104.

Orang-utan, r73, $385 \mathrm{ff}$.

Ornithorynchus, 377 .

Orthogenesis, 24 ff., $43,45,48$.

Osborn, H. F., r34.

Ostrich, 373.

Otolith, rog.

Ova, 23, 67, Ir 5 ff., 366 ff.

Oviparity, 366, 373 .

Owl, 374 .

$\mathrm{Ox}, 380$.

Oxygen, 8, 13, 121.'

Ozone, 13.

\section{$\mathbf{P}$}

\section{Pagano, 229.}

Pain, 275 ff., 284 ff., $29 \mathrm{r}$ ff.

Pangene, 39.

Pangenesis, 35 .

Panmixia, 42.

Paramecium, III ff., 121 ff., 284, 289.

Parasitism, 342, 35r, 355 ff.

Parental care, 203, 243, 253, 336, 344, $348,350,367$ ff., 370 ff., 374,397 ff.

Parental instincts, 337,342 ff., 364 ff., $397 \mathrm{ff}$.

Parker, G. H., I03, I40, I43 ff., I47 ff. 202.

Parrot, 374.

Parthogenesis, 43.

Pastoral ant, 346,347 ff., 357 .

Paternal care, 367 ff., 373.

Pearson, K., 35, 58, 6r, 73.

Peckham, Mr. and Mrs., 233, 268.

Penguin, 412.

Periodic law, 8.
Perrier, E., 329.

Personality, 307 ff.

Petrucci, R., 329 ff., 332 ff., 388, 4I 2, $413,417,421$.

Peuplade, the, $403 \mathrm{ff}$.

Pheasant, 373.

Philoprogenitive instincts, see reproductive instincts.

Photopathy, $92 \mathrm{ff}$.

Phototaxis, see phototropism.

Phototropism, $88 \mathrm{ff}$.

Phrenology, r75, 179, 180 .

Physics, 7 .

Physiognomy, 175 .

Physiological division of labor, 328, 335, 339 ff., 363 .

Physiological state, I3I ff., 136.

Physiological unit, 39 .

Pigeon, 233, 373.

Placentalia, 378.

Planarian, 96, 106.

Plastidule, 39.

Platypus, 377.

Play, 242, 248 ff.

Pleasure, 275 ff., 284 ff., $29 \mathrm{r}$ ff.

Polyandry, 387,401 .

Polygyny, 385, 387, 40r.

Polymorphism, 339 ff., 363 .

Polyp, 328.

Polyphyletism, 39r ff.

Pons Varolii, 167, 228.

Porthesia chrysorrhexa, ro6.

Primate, 27 r, 272, 280, 333, 384 fi.

Promiscuity, sexual, 387 .

Protophyta, 23.

Protoplasm, 16 ff., r 18 ff., 138 ff., $146,274$.

Protozoa, 118 ff., 127 ff., 138,328 .

Psychology, 2, 3, 69 ff., 77 ff., 325 ff.

Psycho-physical interactionism, 322.

Psycho-physical parallelism, 278, 285 ff., 321 ff.

Pugnacity, 24I ff., 249, 25I, 302.

Pure lines, $6_{4} \mathrm{ff}$.

Q

Queen ant, 343, 357, 358.

$\mathbf{R}$

Rabbit, 380.

Rabl-Ruckard, 360.

Raccoon, 38r, 382.

Radioactivity, ro. 
Radium, ro.

Read, Carveth, 229, 233 ff.

Reason, 198, 279 ff.

Receptor, I64 ff., I 7 I, I 73, 280.

Recognition, 337,360 ff.

Reënforcement, 2 I I, 21 7, 275 ff., 305, 3 I 2, $407 \mathrm{ff}$.

Reflex, I 28 ff., I40 ff., I59 ff., I87, I9I, I97, I 98 ff., 256, 259, 289, 330, 335 .

Regression, 62.

Religion, 4I4.

Reproduction, 2 I ff., 335 ff.

Reproductive instincts, $35^{2}$ ff., $35^{8}, 363$, 397.

Reptile, 157, 268, 333, $37 x$ ff., 376.

Repulsion, 244.

Respiration, 20.

Reversion, 59 ff.

Rhea, 373.

Rheotropism, $\mathrm{II}_{4} \mathrm{ff}$.

Rhinoceros, 380.

Richet, C., 79, 80.

Rivalry, 24I, 25 I.

Rodentia, 333, 379.

Romanes, G. J., 28, 34, I98, 201, 204, 215 ff., $286,315$.

Royce, J., 316, 320, 321, 323, 324, 325 .

Rut, the, 399, 4I 2, 4I3, 414 .

Rutherford, E., I 2, 323.

\section{S}

von Sachs, J., 90 , 9r, I5r.

Salamander, 370 .

Salmon, 4I 2.

Santee, H. E., 178, 179, I83 ff., I92.

Schiller, F., 248.

Seal, 38I, 382.

Secretiveness, 242.

Selection, 33 ffi., 40 ff., I 34, 213, 253, 383 .

Selenotropism, 89.

Self-abasement, 244 .

Self-assertion, 244 .

Self-consciousness, $307 \mathrm{ff}$.

Sensation, 180 ff., 274, 280, 290 ff.

Sensori-motor, 225, 275.

Sex, 65 ff., 335 .

Sexual dimorphism, 339 .

Sexual instinct, 210,242 ff., 253,336 , 337,364 ff., 296 ff., 399 ff.

Shame, 242.

Shark, 294, 369.

Sheep, 380 .

Sherrington, C. S., r 43 ff., I45 ff., r56, Tarsius spectrum, 384 fi.:
160, I6I ff., 163, 164 ff., 17ז, I73, 202, 206, 302, 303, 304, 305 .

Shyness, 242.

Simiida, 385, 39r.

Sirenia, 379.

Slave-making ant, 357 ff.

Smell, III, 36I, 366.

Smith, Adam, 4I8, 420, 421.

Snake, 37 r.

Snyder, Carl, I7, I8.

Sociability, 242, 252 ff., 392.

Society, 328 ff., $390 \mathrm{ff}$.

Sociology, 2, 3, 69 ff.

Sparrow, 374.

Species, origin of, $23 \mathrm{ff}$., 45 .

Spencer, H., 14, I5, 39, I68, I 7 I, 199 ff., 202, 224, 248, 298, 301, 3I5.

Spermatozoa, 23, 67, II 5 ff., 366.

Sphenodon, 373.

Spiny anteater, 377.

Spurzheim, I 79.

Stentor caruleus, $95 \mathrm{ff}$.

Stentor roselii, I $24 \mathrm{ff}$.

Stereotropism, I 3 ff., 370.

Stickleback, 368.

Stimuli, 85 ff., I32, I45, I50 ff., I64 ff., 210.

Stirps, 39.

Stout, G. F., 225, 278, 282, 297.

Strasburger, E., 90.

Stridulation, 362 .

Structure, 206 ff., 264, 269, 324.

Struggle for existence, $33,36,4 \mathrm{I}$ ff., 213 , 392.

Subatomicity, 9 .

Subconscious self, 310 ff.

Sucking reflex, 203.

Suggestion, $247,336,355,359,362 \mathrm{ff}$., 375,407 .

Sutherland, A., 366, 369, 398, 418, 419, 421.

Symbiosis, 347 ff.

Symmetry, 27, 29, 47 ff., 93, II2, 269.

Sympathy, 242, 247, 266 ff., 4I8 ff.

Symphile, 352, 353 ff., 355 .

Synapse, I 55 ff., 276.

Synechthran, 352 ff., 355 .

Synoekete, 352, 353, 355 .

\section{$\mathbf{T}$}

Tait, P. G., 37 .

Tarde, G., $42 \mathrm{I}$. 
Taste, III.

Taxis, 84 .

Termite, 339, 357 .

Theories of social evolution, $4 \mathrm{I} 6 \mathrm{ff}$.

Thermotropism, I 6 .

Thigmotropism, $\mathrm{II}_{3} \mathrm{ff}$.

Thomson, J. A., 34, 35 .

Thorndike, E. L., 268, 276, 407.

Thought, 277 ff.

Tiger, 254, 392.

Titchener, E. B., 278, 287, 32 I.

Toad, 370.

Tonotropism, 116.

Tool, 27 I, 272.

Torrey, H. B., $97 \mathrm{ff}$.

Tortoise, $37 \mathrm{r}$.

Tradition, 273, 337, 375, 4II.

Transient instincts, 213 .

Tropism, 81 ff., 88 ff., 136, 197, 198 ff., 256, $259,284,289,330$.

Tschermak, 55 .

Turtle, 371 .

\section{$\mathbf{U}$}

Ungulata, 378,380 ff., 391 .

Urea, 13.

Utility for survival, 392.

\section{V}

Variation, $32 \mathrm{ff}$., $36 \mathrm{ff}$., $40 \mathrm{ff}$., $46 \mathrm{ff}$., $68 \mathrm{ff}$., I34, 213.

Vascular or vasometer system, $300 \mathrm{ff}$., $328,372$.

Veblen, T., 252.

Vertebrate, 156 ff., 268 ff., 288 ff., 294, $295,329,333,364$ ff.
Verworn, M., I7, 39, 84, 86 ff., 9I, II3 ff., II6, II7, I4I ff.

Viscera, $300 \mathrm{ff}$.

Vision, 105.

Viviparity, 366 ff., 370.

Volition, 3 I I ff.

de Vries, H., 39, 46, 5 I ff., 55, 59, 60, 65.

\section{W}

Wagner, 34 .

Waldeyer, I54.

Walrus, $38 \mathrm{r}$.

Ward, J., 320.

Ward, L. F., 42 r.

Washburn, Margaret F., 81, 84, II6.

Wasmann, E., 268, 35 I, 36r.

Wasp, 233 ff., 268, 338 ff., 392.

Weasel, 381,382 .

Weismann, A., 38 ff., 53, 64, 34I.

Wheeler, W., M., 268, 294, 339 ff., 34I, $342,344,346,347,352,354,357,360$.

Whetham, W. C. D., Io, 324 .

Whitman, C. O., 233.

Wilder, H. H., I56.

Will, 3 I I fi.

Woodpecker, 374 .

Woodworth, R. S., 278.

Worker ant, 34I ff., 343 ff.

Workmanship, 252.

Worm, 233, 293, 328.

Writing, $27 \mathrm{r}$.

Wundt, W., I53, I54, I60, I69, I7I, I72, I78, 180 ff., 278.

\section{Y}

Yerkes, R. M., 206, 325. 

THE following pages contain advertisements of books by the same author or on kindred subjects 



\section{The Principles of Anthropology and Sociology in Their Relations to Criminal Procedure} Cloth $\quad$ I2mo $\$ 1.25$

Mr. Parmelee has undertaken, in this interesting book, to make available for practical use some of the results obtained by European students of criminology, whose methods are far in advance of those in this country. The fallacy of considering the moral responsibility of the offender as the test of criminality instead of the dangerousness of the criminal to society, is shown in a straightforward treatment of the present methods of criminal procedure, their weakness and abuses. His reasons for the abolishment of obsolete methods of examination and trial by incompetent authorities; for the appointment of specially trained judges and examiners, and the necessity for scientific study of criminal procedure, are clear, convincing, and enlightening. The relations of heredity and environment, the necessity of individual treatment with the view of reform wherever possible; the suspension of sentence and the probation system; methods of repressing crime, and the miscarriage of justice through the technicalities of the courts, are subjects that are of vital interest to every reader.

Mr. Parmelee has drawn a vivid though unbiased picture which will be a revelation to the man who would know, and he has forcefully outlined the new methods necessary for the improvement of our present system.

"The discussion is in every way strong and clear, and deserves the careful study of all intelligent citizens."-The Dial.

\section{THE MACMILLAN COMPANY

Publishers 64-66 Fifth Avenue New York




\title{
The Animal Behavior Series
}

UNDER THE GENERAL EDITORSHIP OF

ROBERT M. YERKES, Pн.D.

Instructor in Comparative Philosophy, Harvard University

The aim of the Series is to present a number of small volumes which taken together shall form a comprehensive introduction to Comparative Psychology.

\section{The Dancing Mouse}

\author{
By ROBERT M. YERKES, PH.D. \\ Cloth, 12mo, $x x i+290$ pages, illustrated, $\$ 1.25$
}

"Dr. Yerkes' book is a most interesting example of modern laboratory methods and can be read with profit by any one, on account of the accurate methods of observation and careful deductive reasoning which it shows. An elaborate, painstaking system of experiments was carried on with over 400 mice, with the object of determining muscular coördination, structural peculiarities, strength of the special sense organs, habit formation, educability, and strength of memory of these little animals. Some experiments were also undertaken along the line of inherited peculiarities. ... The work is really only a preliminary study, but it will be read with much interest by all students of comparative psychology." Journal of American Medical Association.

\section{The Animal Mind}

\section{By MARGaRET FLOY WASHBURN, Ph.D. Professor of Philosophy, Vassar College}

$$
\text { Cloth, 12mo, } 333 \text { pages, } \$ 1.60
$$

"As the author points out, the title of this book might more appropriately have been 'The Animal Mind Deducted from Experimental Evidence,' for the facts here set forth are very largely the results of the experimental method in comparative psychology. The mass of experimental material that has been accumulated from the researches of physiologists and psychologists is always great, but is also for the most part inaccessible to the ordinary student, being widely scattered and to a considerable extent published in the journals of specialists, which the average college library does not contain. Hence the usefulness of the present ... interesting work." - New York Sun.

\section{THE MACMILLAN COMPANY}

Publishers 64-66 Fifth Avenue Now York




\title{
THE ANIMAL BEHAVIOR SERIES
}

EDITED BX PROFESSOR ROBERT M. YERKES

\section{ANIMAL INTELLIGENCE EXPERIMENTAL STUDIES}

\author{
By Professor EDWARD L. THORNDIKE
}

Teachers College, Columbia University

Cloth $\quad 297$ pages $\$ 1.60$

In this volume are brought together the principal experimental studies of animal intelligence made by Professor Thorn dike, and a discussion of the general laws of animal intellect and behavior, which have been demonstrated by recent work in animal psychology. These studies have a twofold interest. In the first place since they represent the first deliberate and extended application of the experimental method in animal psychology, they are a most useful introduction to the later literature of that subject. They mark the change from books of general argumentation on the basis of common experience interpreted in terms of the faculty psychology, to monographs reporting detailed and often highly technical experiments interpreted in terms of original and acquired connections between situation and response.

In the second place, since they represent the point of view and the method of present animal psychology, but in the case of very general and simple problems, they are useful also as readings for students who need a general acquaintance with some samples of experimental work in this field.

The work includes chapters on The Study of Consciousness and the Study of Behavior; Animal Intelligence; The Instinctive Reactions of Young Chicks; The Psychology of Fishes; The Mental Life of the Monkeys; Laws and Hypotheses of Behavior, and The Evolution of the Human Intellect.

\section{THE MACMILLAN COMPANY Publishers




\section{BY EDWARD A. ROSS}

\section{Professor of Sociology, University of Wisconsin}

Formerly of the University of Nebraska

\section{Social Psychology}

Cloth, 8vo, \$I.50

"The volume marks off for itself a very definite field of research, and scours the circumscribed area in the most scientific manner. . . . Professor Ross has laid bare the more vital social traits, good and bad, of the human mind in a manner calculated to awaken thought." - The New York Tribune.

\section{Social Control}

\section{A Survey of the Foundations of Order}

$$
\begin{aligned}
& \text { Part I - The Grounds of Control } \\
& \text { Part II - The Means of Control } \\
& \text { Part III - The System of Control }
\end{aligned}
$$

Cloth, r2mo, leather back, $\$ 1.25$

The author seeks to determine how far the order we see about us is due to influences that reach men and women from without, that is, social influences. His thesis is that the personality freely unfolding under conditions of healthy fellowship may arrive at a goodness all its own, and that order is explained partly by this tendency in human nature and partly by the influence of social surroundings. The author's task, therefore, is first to separate the individual's contribution to social order from that of society, and second, to bring to light everything that is contained in this social contribution.

\section{Foundations of Sociology}

The Scope and Task of Sociology - The Sociological Frontier of Economics - Social Laws - The Unit of Investigation in Sociology - Mobmind - The Properties of Group-Units - The Social Forces - The Factors of Social Change - Recent Tendencies in Sociology - The Causes of Race Superiority - The Value Rank of the American People.

The author lays a foundation for a social science that shall withstand the severest tests, and formulates the principal truths about society. In the belief that the time is past for one-sided interpretations of society, the effort is made to group together and harmonize the valuable results of all the schools.

Both the above are in "The Citizen's Library" of Economics, Politics, and Sociology, edited by RIChakD T. ELY, Ph.D., LL.D., Professor of Political Economy, University of Wisconsin.

\section{THE MACMILLAN COMPANY}

\section{Publishers 64-66 Fifth Avenge Jow York}








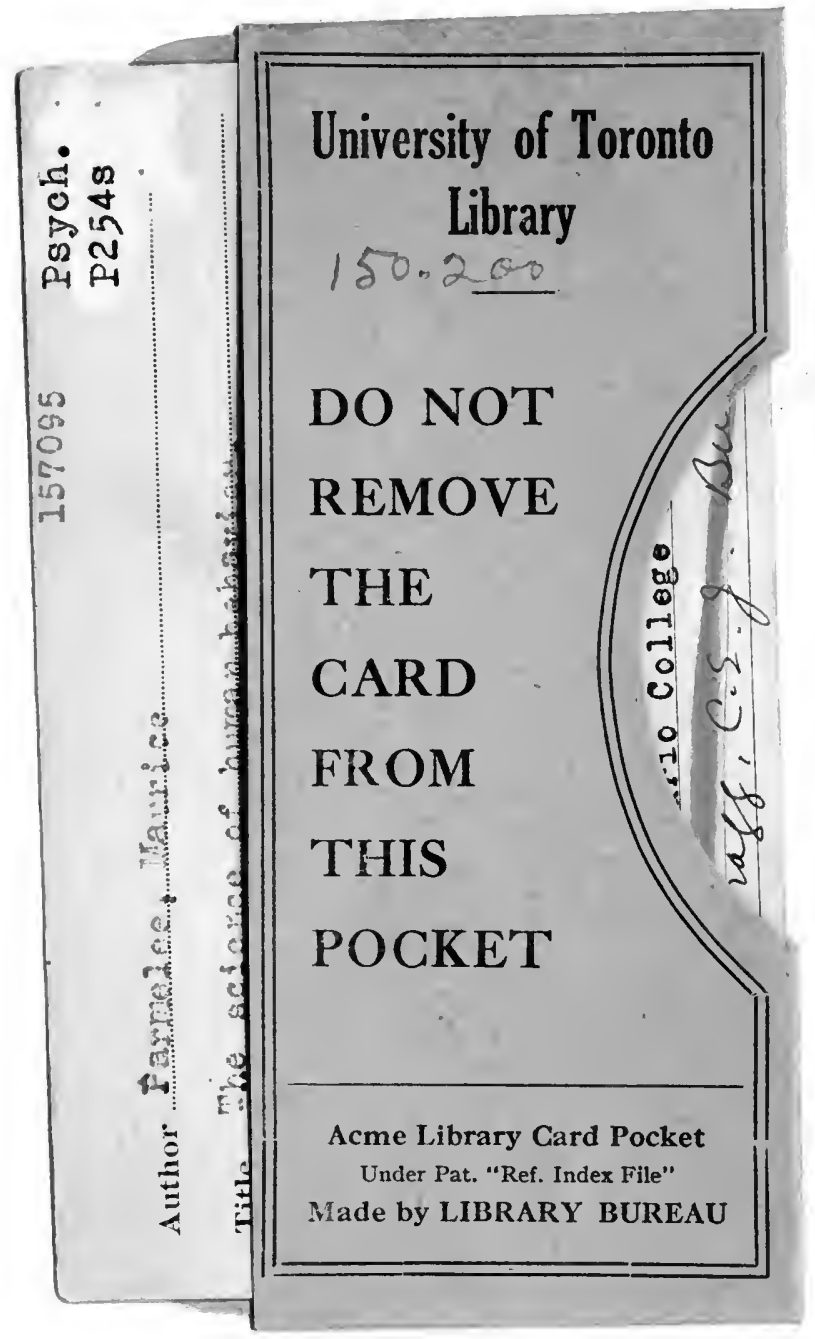




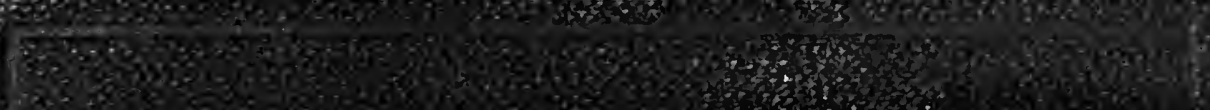
(S)

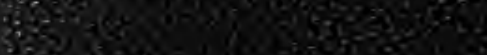

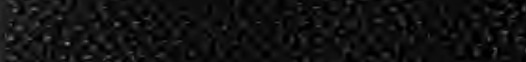

3.

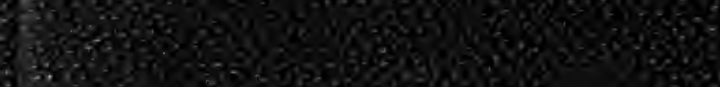

3.

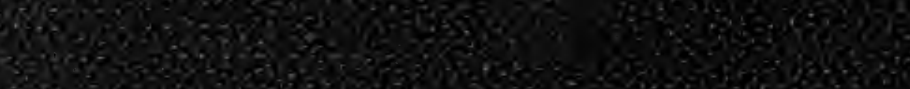

-

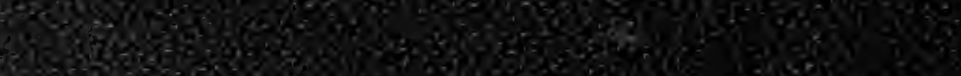

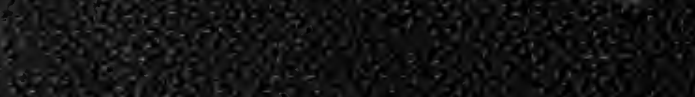

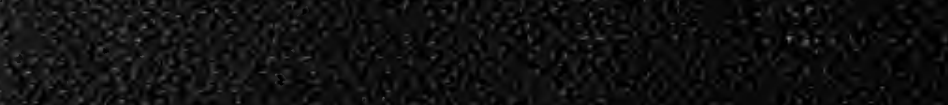

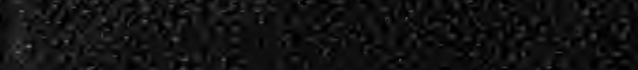

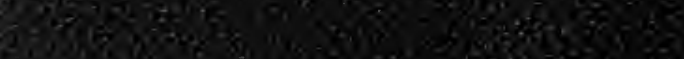

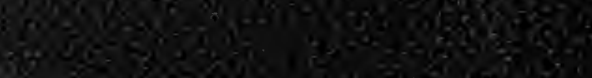

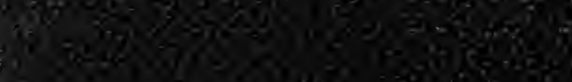

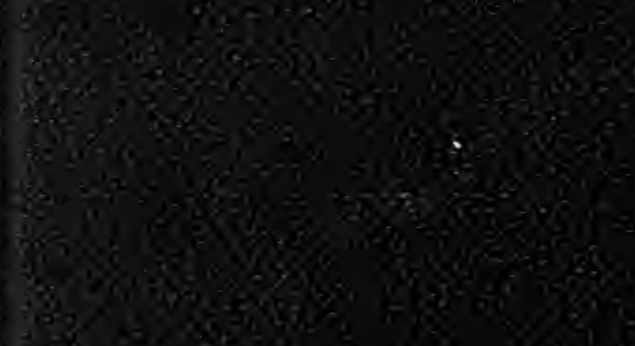

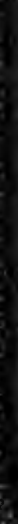

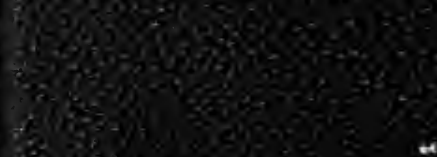
38078

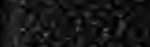

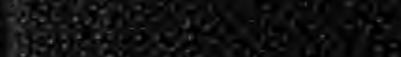

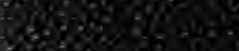

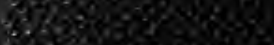

2i:

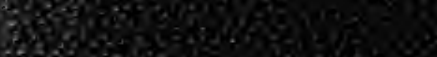

